\title{
Abstracts of the Biomedical Engineering Society 1993 Annual Fall Meeting, October 21-24, 1993
}

\author{
Cardiopulmonary Engineering
}

\author{
A MODEL OF THE PULMONARY ARTERIAL SYSTEM \\ B.R. WIGGS* \\ MIT Fluid Mechanics Lab, Dept. Mechanical Engineering, Cambridge, MA
}

The anatomical structure of the human pulmonary circulation is extremely complex. Previous work by several authors have used various branching schemes to represent this branching system of vessels. In this work the orginal cast data of Singhal (JAP:33:190, 1973) was used to generate an anatomically based model of the arterial branching network which could be used to estimate red blood cell. The data were found to contain many trifurcations in addition to the bifurcating branches. Other investigations of arterial trees have used systems which could not accurately predict the path length travelled and hence could not obtain transit time estimates. The model is a set of probabilities governing whether a division bifurcates or trifurcates and how rapidly a given vessel approaches the capillary bed. When coupled to a microvascular model of the pulmonary capillary bed, this large vessel model (which can be slightly altered to simulate the venous vessels) estimates red blood cell transit times and pressure drops similar to those recorded in human experiments.

*Fellow of the American Lung Association
MORPHOMETRY OF THE HUMAN PULMONARY ARTERIAL AND VENOUS TREES Wei Huang, Matt McLaurine, Gary Bledsoe, and Michael R. T. Yen Department of Biomedical Engineering, Memphis State University, Memphis, TN 38152

A complete analysis of pulmonary blood flow in the lung must take into account the detailed vascular branching pattern and vascular topography. So far, the data of human pulmonary arterial and venous trees is based on the dimensional model using Strahler's ordering system by Horsfield(1978). In the Strahler system the greatest difficulty is broad overtap of the range of diameters of successive orders, which greatly reduces the usefulness of the data for hemodynamic analysis. To remedy these difficulties, we used a DiameterDefined Strahler system developed by Kassab and Fung(1992). In the Diameter-Defined Strahler system the trees were ordered according wo diameter. This process is an iterative one whereby Strahler's ordering systern is used initially to order the tree and consequently provide the diameter distribution for each order. The ranges of these diameter distributions are then used to conect Strahler's orders. The connectivity of blood vessels from one order to another is expressed in the connectivity marnix.

We have studied the morphologic pant of human pulmonary arterial and venous trees using cadaver lungs. The human specimens were obtained and cast by a method of silicone elastomer within 6 hours postmortem. Data on order number, diameter, diameter ratio, length, length ratio, and branching ratio are provided. The connectivity matrices of the arterial and venous trees are presented. An entire morphologic circuit of the human pulmonary circulation will be constructed by combining the data of the pulmonary arterial system, venous system, and microvasculature.
A THREE-DIMENSIONAL VASCULAR MODEL ACCOUNTS FOR THE SPATIAL CORRELATION OF REGIONAL PULMONARY PERFUSION Robb Glenny and H. Thomas Robertson. Department of Medicine, University of Washington, Seattle WA

Pulmonary perfusion is spatially correlated, with neighboring regions of lung demonstrating positive flow correlations and distant pieces exhibiting negative correlations. While local correlation has been noted in a wide variety of natural processes, negative correlation has not been observed. We investigated a 3 dimensional branching vascular model to determine if such a model could explain the negative correlation of perfusion. The distribution of flows was modeled by a dichotomously branching tree in which the fraction of flow from parent to daughter branches was $\gamma$ and $1-\gamma$ at each successive bifuncation. The flow asymmetry parameter, $\gamma$, was randomly chosen for each bifurcation from a normal distribution with a mean of 0.5 and a standard deviation of $\sigma$ Regional perfusion in this model was positively correlated locally and negatively correlated at distance. The model was refined by incorporating an effect of gravity and redirecting a fraction, $\delta$, of the flow against gravity to the dependent daughter branch. A flow bias in the "dorsal" direction was also introduced to account for differences in supine-prone perfusion gradients. Sensitivity analyses were performed to determine values of $\sigma$ and $\delta$ which best characterized the spatial perfusion distributions observed in experimental animals $(\sigma=0.07, \delta=0.06)$. A wide variety of observations relating to the spatial distribution of pulmonary perfusion can be accounted for with this 2 parameter model. A 3-dimensional model can relate the function and structure of the pulmonary vascular tree, offering an explanation for both heterogeneous and spatially correlated regional flows. Supported by ALA-Research Grant and NIH HLO2625.

4

HOMOGENEITY AND HETEROGENEITY IN PULMONARY HEMODYNAMICS Gary S. Krenz, Christopher A. Dawson, Jianming Lin, and John H. Linehan. Marquette University, Medical College of Wisconsin and Zabbeki VAMC, Miw. WI

Direct observation of intrapulmonary arteries using microfocal angiography yields voluminous data. The scale invariant or fractal appearance of the arterial tree suggests a simplitication. The diameter, $D$, of each vessel at a biturcation is measured. By assuming a homogeneous arterial tree, Poiseuille flow and an average length to diameter ratio, $r$, an expression for the (from arterial inlet to capillary inlet) cumulative resistance, $R_{c \leq m}$ as a function of cumulative vascular volume $Q_{c u m}$ is

$$
R_{\text {cum }}=R_{a} \frac{\left[\frac{Q_{\text {cum }}}{Q_{a}}\left(r^{\beta_{1}-\beta_{2}-2}-1\right)+1\right]^{\left(-\beta_{1}-\beta_{2}+4\right) /\left(\beta_{2}-\beta_{2}-2\right)}-I}{r^{-\beta_{2}-\beta_{2}+4}-1}
$$

where $R_{\mathrm{a}}$ denotes resistance of the first vessel, $Q_{a}$ its volume, and the parameters $\beta_{1}$ and $\beta_{2}$ are $\log \log$ slopes. Hemodynamic consequences of the tree structure are expressed by $\beta_{1}$. A value of 2.5 is suggested by control morphometric data for $\beta$, The resulting expression for $R_{c u m}$ as a function of $Q_{c u m}$ is then concave upward. This results in the interpretation that "small" arteries act as the resistance vessels. The concavity persists when, instead, related heterogeneous random tree structures are examined. Using microfocal angiography and these models, the effects of vasoactive agems upon $\beta_{1}$ and hence the distribution of nemodynamic resistance within the pulmonary arterial tree can be examined.

This work was supported by HL-19298 and the Department of Veterans Affairs. 
MEASUREMENT OF PULMONARY ARTERIOLAR AND VENULAR DISTENSIBILITY USING VIDEO MICROSCOPY. W.W. Wagner, Ir., C.C. Hanger, S.C. Hillier. Indiana University Medical School, Indianapolis, IN.

Pulmonary vascular distensibility has an important influence on pulmonary hemodynamics. To obtain information on the distensibility of microvessels, video microscopy was used to record subpleural arterioles and venules $<100 \mu \mathrm{m}$ as transmural pressure was varied form $5-30 \mathrm{mmHg}$. To accurately determine the edge of the red cell column, a computer digitized 30 video frames. Pixeis that changed between frames were tumed white; pixels that did not change between frames were turned black. In this way, moving erythrocytes could be distinguished from the stationary vessel wall. By adding together 30 of these enhanced images, a single image containing a distinct vessel lumen was obtained for each transmural pressure. A set of computer generated lines was placed by the operator just within the boundary of the enhanced vessel lumen. The computer moved each line outward. Pixels just outside each moving line were compared to pixels just inside the line. When the line had progressed past the vessel margin, line movement was stopped and the computer repositioned them back to the location of maximum difference between the inside and outside of the line. The diameter of the vessel was taken as the orthogonal distance betwoen the lines. This technique has produced the first measurements of individual microvessels over a transmural pressures in the physiologic range. The distensibilities of arterioles and venules were similar and were also similar to larger pulmonary arteries and veins. Supported by NIH HL 36033
THE MECHANICAL PROPERTIES OF THE HUMAN MAIN PULMONARY ARTERY Matthew McLaurine, Wei Huang, and Michael R. T. Yen.

Department of Biomedical Engineering, Memphis State University, Memphis, TN 38152

Understanding of the pressure-flow relationship of the blood in the pulmonary circulation is vital for understanding both normal and pathological conditions of the lung. In order to describe this relationship, one must realize the factors that are influencing it, such as: the elasticity of the blood vessels, the rheology of the blood, and the morphometry of the vaculature. Data on human pulmonary vascular elasticity is sparse at best, for most elasticity studies have concentrated on species other than the human. With this data of the human pulmonary vascular elasticity, we will be one step closer to realizing a precise working model of the human lung.

For the purpose of obtaining the mecharical properties of the main pulmonary artery, and comparing how they change due to age, the material constants involved in the constitutive equations for finite deformation were obtained. All samples were taken within six hours of post-mortem, and were from three age groups: young(under 30 years), middle( $30-50$ years), and old(over 50 years). The analysis of suress and strain under the assumption that the unloaded cylinder is the zero stress state incurs error; therefore, we have incorporated the zero stress state into the studies of the mechanical properties of the vessel. The specimens were cut longitudinally and circumferentially and tested in a uni-axial fashion. After pre-conditioning was performed on each specimen, we tested for the frequency depedence of elasticity and found that it was rather insensitive to the strain rate. Therefore, we employed the pseudoelasticity concept to describe the stress-strain relationship in specific cyclic loading. Using the Instron 8500 Dynamic Testing System, very high resolution tests were performed and the aforementioned properties were obtained. 
7

DIRECT MEASUREMENTS OF PULMONARY CAPILLARY TRANSIT TIME IN RABBITS

S. J. Lai-Fook, P. M. Wang, I. Ayappa, L. V. Brown, M. Jahed.

Center for Biomedical Engineering, University of Kentucky.

Fluorescence videomicroscopy was used to record the passage of fluorescent dye through the subpleural microcirculation of anesthetized rabbits and isolated perfused rabbit lungs (J. Appl. Physiol. 69:2262, 1990; 72: 2420, 1992). During a 2 minute apneic period in vivo, dye was injected into the right ventricle and the dye passage through the microcirculation was observed through a transparent parietal pleural window and recorded on videotape. Arteriolar and venular dye dilution curves were cbtained from the video images using digital imaging processing. Capillary transit time computed from the mean time difference between the 2 curves averaged $0.6 \mathrm{~s}$ at FRC and increased 4 fold as airway pressure increased from 0 to $20 \mathrm{cmH}_{2} \mathrm{O}$ and cardiac output decreased 3 told. In the isolated lung dye dilution curves were measured from the video signals using a video photometric analyser. A lagged normal density curve was used to fit the a dideo phomes of anput art A lagged normal density curve was used to fit the dye dilution curves of inpul arterlal, arentar, venular and output venous tlows, and to calculate capillary transit time and relative rabbin lung, capillary transil time decreased with blood flow and lung inflation but increased with left atrial pressure. Relative flow dispersion was smaller $(\sim 0.5)$ in the arterial segment than in either the capillary or venous segment $(>0.9)$. Similar large dispersion values have been tound in the dog lung capillaries (FASEB J. 7 A788, 1993). Extrapolation of the measured capillary transit time to high cardiac outpu's suggests that with heavy exercise capillary transit time would be reduced to values low enough to cause $\mathrm{O}_{2}$ desaturation. Compensation can occur by reducing lung volume or increasing left atrial pressure. Supported by HL.36597.

MATHEMATICAL MODEL FOR REGIONAL TRANSIT TIME AND FLOW ESTIMATION FROM MICROFOCAL ANGIOGRAPHIC IMAGES. A.V. Clough, A.T. Cheng, A. Al-Tinawi, J.H. Linehan, C.A. Dawson: Marquette Univ., Med. Coll. of Wisc., Zablocki VAMC, Milwaukee, WI

Methods for estimating regional mean transit time $(\tau)$ and flow $(F)$ within a region-of-interest (ROI) using digital angiography involve measuring time-absorbance curves for an inlet artery and a microvascular ROI. We examined the ability of several methods to recover $\tau$ using a computer simulated network of vessels representing that which might occur in a real organ. Concentration curves measured over the inlet to the network and ROI residue curves were simulated. When the height-area ratio of the $\mathrm{ROI}$ residue curve was used and all of the indicator was contained within the ROI for at least one sample time, $\tau$ was recovered exactly. As the ROI was reduced or the inlet concentration curve became more dispersed, the error in the recovery of $\tau$ increased. By first deconvolving the inlet concentration curve from the ROI residue curve, and then calculating the height-area ratio of the resulting impulse residue curve, $\tau$ was recovered exactly. Estimates of $\mathrm{F}$ were obtained simultaneously. The methods were applied to microfocal $x$-ray angiography data from a $~ 700$ $\mu \mathrm{m}$ canine pulmonary artery and an ROI over the surrounding microvasculature. Estimates of $\tau$ and $F$ were obtained using the methods described above. The robustness of the methods and the ability of the computer network model to simulate the pulmonary microcirculation was verified. Supported by HL-19298 and Dept. of Veteran Affairs.

\section{9}

PULMONARY CAPILLARY TRANSIT-TIME DISTRIBUTION

Audi, S.H., G.S. Krenz, J.H. Linehan, D.A. Rickaby and C.A. Dawson

Marquette University; Medical College of Wisconsin and VAMC, Mitwaukee, WI 53295

Let $h(t)$ be the probability density function of transit times. The following equations relate the moments (mean transit time, $\tilde{\mathrm{t}}$, variance, $\sigma^{2}$, and skewness, $\mathrm{m}^{3}$ ) of the capillary function, $h_{c}(t)$; of the extravascular function, $h_{e}(t)$, and of the noncapillary function, $h_{n}(t)$, to the moments of the venous concentration versus time curves for a vascular reference indicator, $C_{R}(t)$, and a flow-limited diffusible indicator, $C_{D}(t)$, following a bolus injection of the indicators upstream from the lung

$\bar{t}_{\mathrm{R}}=\bar{t}_{n}+\bar{t}_{\mathrm{c}} ; \sigma_{\mathrm{A}}^{2}=\sigma_{\mathrm{n}}^{2}+\sigma_{\mathrm{c}}^{2} ; m_{\mathrm{A}}^{3}=m_{\mathrm{h}}^{3}+\mathrm{m}_{\mathrm{z}}^{3}$

$\bar{t}_{D}=\bar{t}_{n}+\left(1+\frac{\bar{t}_{\theta}}{t_{c}}\right) \bar{t}_{c} ; \sigma_{D}^{2}=\sigma_{n}^{2}+\left(1+\frac{\bar{t}_{\theta}}{\bar{t}_{c}}\right)^{2} \sigma_{C}^{2} ; m_{B}^{3}=m_{n}^{3}+\left(1+\frac{\bar{t}_{e}}{\bar{t}_{c}}\right)^{3} m_{c}^{3}$

The moments of $h_{c}(t)$ can be estimated if the injected bolus includes, along with the vascular reference indicator, at least two flow-limited diffusible indicators each with a different $\tilde{t}_{\theta}$. A least squares optimization procedure can then be used to specify the moments of $h_{C}(t)$. This approach was applied to isolated dog lung lobes with $\left[{ }^{14} \mathrm{C}\right] d i a z e p a m$ as the diffusible indicator. The tissue-to-pertusate partition coefficient for $\left[{ }^{14} \mathrm{C}\right]$ diazepam could be adjusted to any desired value by allering the perfusate albumin concentration. Thus, by making two injections at different pertusate albumin concentrations, data were obtained in a manner equivalent to making one injection with two flow-limited diffusible indicators each with a different $\bar{t}_{\theta}$. The estimated capillary mean Iransit time (blood volume) was about $48 \%$ of the total lobar mean transit time (blood volume), and the relative dispersion of the helt) was about $75 \%$.

Supported by the Department of Veterans Affairs and NHLB! grant HL24349.
10

A MATHEMATICAL MODEL WITH EXPERIMENTAL VALIDATION OF PULSATILE FLOW IN THE CATS LUNG

Yuan Tian, Wei Huang, and Michael R. T. Yen

Department of Biomedical Engineering. Memphis State University, Memphis, TN 38152

The present investigation is part of our effort to better understand the hemodynamic behavior of the pulmonary circulation. A mathematical model of the entire vascular system of cat's lung was developed. This model was based on the detailed branching patterns and elasticity of the pulmonary vascular bed which were measured in our laboratory to predict the essential features of the physical system with respect to pulmonary vascular input impedance. In our studies, we separated the pulmonary vasculature into two basic components, the anerial and venous vessels and the capillary network. The arteries and veins are treated as elastic tubes and the capillaries as two-dimensional sheets. For the puimonary arteries and veins. Womersley's equation for a constrained elastic tube was used for each segment of a branching system. For the pulmonary capillaries, a more realistic model based on the sheetflow theory proposed by Fung and Sobin was used. With these treatments, we can synthesize macro- and microcirculation into a circuit.

In order to validate this model, a series of experiments on isolated perfused cat lungs were carried out to examine the relationship of pulsatile blood pressure and flow. In the were carried out to examine the relationship of pulsatile blood pressure and flow. In the
experiments, the pulmonary proximal and distal arerial pressure, pulmonary venous pressure, experiments, the pulmonary proximal and distal anerial pressure, pulmonary venous pressure, and pulmonary arterial flow were measured. The pulmonary vascular input impedance, the
wave velocity of the pressure, and potential and kinetic hydraulic energy in the main wave velocity of the pressure, and potential and kinetic hydraulic energy in the main
pulmonary artery were determined using Fourier series method. We found that the input impedance spectra were similar in forms to those in many other studies and were consistent with the theoretical prediction.

11 MORPHOLOGIC AND FUNCTIONAL RELATIONSHIPS IN THE LAMB MAIN PULMONARY ARTERY

B. Ha, C. F. Zhao, ${ }^{1}$ R. Zalesak, ${ }^{1}$ H. Katayama, ${ }^{2}$ R. Krzeski, ${ }^{3}$ J. L. Ferreiro, ${ }^{3}$ G. W. Henry, ${ }^{3}$ P. Lynch, ${ }^{4}$ A. P. Yoganathan, ${ }^{4}$ and C. L. Lucas. ${ }^{1}$

Departments of Surgery, Biomedical Engineering, ${ }^{1}$ and Pediatrics, ${ }^{3}$ UNC Schoolef Medicine, Chapel Hill, NC 27599-7065. Department of Pediatrics, ${ }^{2}$ Towakai Hospital, Osaka, Japan. Biofluid Dynamics Laboratory, ${ }^{4}$ Georgia Institute of Technology, Atlanta, GA 30332-0100.

Quantitating the geometric features of the main pulmonary artery bifurcation has been shown to be useful in the assessment of pulmonary hypertension and in understanding the development of abnormal blood flow patterns such as flow separation, secondary flow, and backflow. In this study, in vivo hemodynamic measurements of nomal lambs ranging from 2 days to 6 months in age were obtained. A computer tomography (CT) scanner was used to scan methyl methacrylate casts of the right heart and pulmonary arteries obtained from the study lambs. Computer programs were developed for extracting the geometric features of the major pulmonary arteries programs were developed for extracting the geometric features of the major pulmonary artcrics
quantitatively from the CT reconstructions. The physiologic and study parameters--mean, quantitatively from the CT reconstructions. The physiologic and study parameters--mean,
systolic, and diastolic pulmonary artery pressures, pressure-strain elastic modulus, length, systolic, and diastolic pulmonary artery pressures, pressure-strain elastic modulus, length,
proximal and distal diameters of the main pulmonary trunk--were used to obtain pulmonary artery distensibility estimates (systolic volume minus diastolic volume divided by average volume). Resuits indicate that a regression model can be used to describe the relationship between the study parameters and age: $\mathrm{Y}=\mathrm{A}+\mathrm{B} \mathrm{e}^{-\mathrm{age} / \tau}$ where $\mathrm{A}=$ value at maturity, $\mathrm{A}+\mathrm{B}=$ value at birth, and $\tau=$ time constant. Estimated pulmonary artery distensibility decreased sharply from $23 \%$ to $14 \%$ from birth until 12 days of age. Values ranging from $23 \%$ to $27 \%$ were reached from 40 days upwards. While these estimates agree closely with those reported by other investigators, the characteristic dip in the profile during the first 12 days has not been previously observed. Our findings suggest that the dip is related to the combined effects of rapid decrease in pulmonary artery elasticity $(\tau=9)$ and the slower rate of growth of pulmonary artery dimensions $(\tau \geq 29)$. The combined approach of in vivo and in situ studies is useful in providing a global understanding of age-related changes in the pulmonary circulation. 
12

THE SITE OF ACTION OF HYPOXIA FROM VASCULAR OCCLUSIONS

Leif D. Nelin, Said H. Audi, John H. Linehan and Christopher A. Dawson

Medical College of Wisconsin, Marquette University, Zablocki VAMC, Milwaukee, wI

To determine the site(s) of action of hypoxia in the neonatal pig, isolated lungs were perfused at a constant flow rate and left atrial pressure, then arterial, venous and double ocelusions were performed. The distribution of the total pulmonary vascular resistance (RL) and the total dynamic vascular compliance (Cdyn) were calculated using a model of the pulmonary circulation consisting of upstream $(\mathbf{C u})$, central ( $\mathrm{Cc}$ ) and downstream (Cd) compliances and resistances upstream (Ru) and downstream (Rd) of $\mathrm{C}_{\boldsymbol{c}}$. In addition, the static vascular compliance (Cst) was measured by venous followed by arterial occlusion and the total yasezlar volume $(Q)$ was measured by dye-dilution.

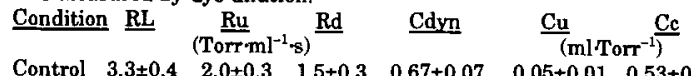

$\begin{array}{llllllll}\text { Control } & 3.3 \pm 0.4 & 2.0 \pm 0.3 & 1.5 \pm 0.3 & 0.67 \pm 0.07 & 0.05 \pm 0.01 & 0.53 \pm 0.05 & 0.07 \pm 0.02\end{array}$

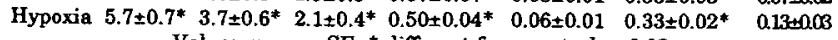
Values mean $\pm \mathrm{SE} ;{ }^{*}$ different from control $\mathrm{p}<0.02$

Hypoxia had no significant effect on Cst $\left(0.9 \pm 0.1 \mathrm{ml}^{-1} \mathrm{Tr}^{-1}\right.$ control, $1.0 \pm 0.1 \mathrm{ml}^{-} \mathrm{Torr}^{-1}$ hypoxia) but significantly decreased $\mathrm{Q}(21 \pm 2 \mathrm{ml}$ control, $17 \pm 2 \mathrm{ml}$ hypoxia, $\mathrm{p}<0.02)$.

These results suggest that in the pulmonary vasculature of the neonatal pig hypoxia 1) changes the viscoelastic properties of the vessels as evidenced by the different response of Cdyn and Cst, 2) results in mainly arterial constriction as evidenced by the large increase in $R u$ and the decrease in $Q$, and 3 ) there is a smaller but significant venous constriction as evidenced by the increase in Rd. This venous constriction may have implications in the pathogenesis and therapy of pulmonary vascular diseases. Supported by HL-19298 and the Department of Veterans Affairs

13

VASOCONSTRICTION HETEROGENEITY OF SMALL PULMONARY VESSELS Al-Tinawi, A., G.S. Krenz, D.A. Rickaby, J.H. Linehan, and C.A. Dawson. Marquette University, Medical College of Wisconsin, and Zablocki VAMC, Milw., wi.

To measure changes in the diameter of small pulmonary vessels (150-1600 $\mu \mathrm{m})$ in response to hypoxia and serotonin $(5-\mathrm{HT})$, we used microfocal x-ray imaging. The response exhibited longitudinal heterogeneity (from arterial inlet to venous outlet) and parallel heterogeneity (among vessels of given control diameler, DC). Longitudinally, hypoxia constricted $<800 \mu \mathrm{m}$ arteries (with the percent constriction dopendent on antery size). Larger arteries were distended as the pulmonary antery pressure increased. $H y$ poxia caused a small constriction in the veins. 5-HT constricted all arteries and had no iniluence on veins. The parallel heterogeneity was revealed as a normally distributed constriction of the vessels of a given $D c$, where $\mu$ is the mean value of $D e / D C$; $D$ is is the experimental diameter, and $\sigma$ is the standard deviation. To value of $\mathrm{De} / \mathrm{DC}$; $\mathrm{De}$ is the experimental diameter, and $\sigma$ is the standard deviation. To address the impact of parallel heterogeneity on the change in resistance of the parallel vessels, we derived the follow-
ing expression relating $\mathrm{Re} / \mathrm{Ac}$ (where $\mathrm{Rc}$ and $\mathrm{Re}$ are the resistances of the parallel vesing expression relating Re/Ac (where Rc and Re are the resistances of the parallel ves-
sels in the control and experimental conditions) to the $\mu$ and $\sigma: R e / R c=$ $1 /\left(6 \sigma^{2} \mu^{2}+\mu^{4}+3 \sigma^{4}\right)$. Thus, for a given value of $\mu$, Re/Rc depends on $\sigma$. The figure shows the overestimation in Re/Rc for a parallel set of vessels having control diameter $\mathrm{Dc}$, if Re were calculated assuming $\sigma=0$, plotted versus the measured $\sigma$. Dashed, solid, and dashed-solid lines represent constriction, no change, and distension, respectively, Open triangie, apen circle, and solid circle represent angere open ing 5 - $\mathrm{HT}$, veins during 5 - $\mathrm{HT}$ and velins durhypoxia size rallects artery. Sold tinangles (triangite size relects artery size) represents arteries 19298 and Dept. of Veterans Affairs.

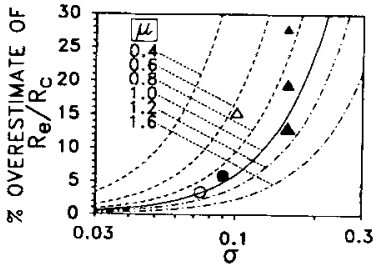

14

\section{PULMONARY ARTERIAL REMODELING IN HYPOXIC HYPERTENSION S.Q. Liu and Y.C. Fung}

University of California San Diego, La Jolla, CA 92093-0412

The effect of pulmonary hyperension on the structure of the rat pulmonary arteries was quantitatively studied by using an electron microscopic method. Pulmonary hypertension was induced in rats by experimental hypoxia (10\% oxygen). At various periods of time (from 2 hours to 10 days), pulmonary arterial blood pressure and cardiac output were determined under the hypoxic environment. The lungs were fixed, samples of the pulmonary aneries were collected, electron microscopic sections were prepated and the areas of the pulmonary arterial components including smooth muscle cells, fibroblasts, collagen fiber bundles, and elastic laminae were determined

The pulmonary arterial blood pressure was rapidly increased during the development of hypoxic hypertension, while the cardiac output remained relatively constant. The areas of the intima and elastic laminae of the pulmonary arteries were increased at 2 and 12 heurs of exposure to hypoxia, decreased to about the control level at 48 hours and increased again afterwards. The areas of the smooth muscle cells and collagen fiber bundles were gradually increased in comparison with corresponding controls, whereas that of the fibroblasts remained relatively stable up to 48 hours and rapidly increased afterwards. It can be concluded that hypoxic hypertension can induce remodeling of the cells and extracellular matrices of the pulmonary areries.
15

THE EFFECT OF LUNG VOLUME ON THE PULMONARY VASCULAR RESISTANCE Haworth, S.T. J.H. Linehan and C.A. Dawson.

Marquette University, Medical College of Wisconsin and Zablocki VAMC, Milwaukee, WI.

The vascular resistance, $R$, versus lung volume, $\mathrm{V}$, curve is $\mathrm{U}$ - or $\mathrm{J}$-shaped with a minimum near the Functional Residual Capacity, FRC. In an attempt to understand the mechanism(s) responsible, we developed a morphometrically based mathematical model. The artery, a, capillary sheet, $c$, and vein, $v$, geometries were as previously summarized by Haworth et al. (J. Appl. Physiol., 70: 15, 1991). Individual artery and vein diameters, $D$, or capillary sheet thickness, $h$, versus transmural pressure, $P(m$, is described by $D=D o \cdot E: h=h o \cdot E, E=$ $\gamma-(\gamma-1) \exp [(-\alpha \mathrm{Ptm}) /(\gamma-1)]$, where $\mathrm{Do}$ and ho are at $\mathrm{Ptm}=0 ; \alpha$, is the distensibility constant and $\gamma$ is the ralio of the maximum $D$ or $h$ to $D o$ or ho, respectively. Prm is the vascular pressure, $\mathbf{P}$, minus perivascular, $\mathbf{P}_{\mathbf{x}}$ pressure. For the arteries and veins, $\mathbf{P}_{\mathbf{x}}=\left(\lambda_{3}(\mathbf{P}+\right.$ $\left.\mathrm{Ptp})+\lambda_{4}\right) \mathrm{Ptp}+\left(\lambda_{3}(\mathrm{P}+\mathrm{Ptp})+\lambda_{1}\right) \lambda_{2}-\lambda_{1}-\mathrm{Ptp}$, where Ptp is the transpulmonary pressure and the parameters, $\lambda_{1}, \lambda_{2}, \lambda_{3}$ and $\lambda_{4}$, are estimated from the data of Smith and Mitzer (J. Appl. Physiol., 48: 450, 1980) or Lai-Fook (J. Appl. Physiol. 46:419, 1979). For the capillary sheet, $P x=$ alveolar pressure. The resistance across each order of arteries or veins, Ra. $v=(128 \mu \mathrm{L}) /\left(\pi \mathrm{D}^{4}\right.$ $N)$; or capiliary sheet, $R c=(12 \mu L f) /\left(w h^{3}\right)$, where $L$, is length, $\mu$, is blood viscosity, $N$, is number of vessels, $w$, is the width of the capillary sheet and $f$, is the sheet geometric friction factor. During lung inflation $V=(d V / d P t p) \cdot P t p+V i$, where $V i$, is the lung volume at the beginning of inspiration. During lung deflation $V=T L C[1-\exp (-\mathbf{k} P t p)]+R V$, where $T L C$ is the total lung capacity; $\mathrm{RV}$ is the residual volume plus tissue volume and $\mathrm{k}$, lung deflation parameter. The $L$ of the $a, c$ and $v$ is assumed proportional to the $1 / 3$ power of $V$. The capillary sheet blood volume at Ptr $=0$ is constant for all $\mathrm{L} w \mathrm{wh}$. The model series arrangement of a $\mathrm{c}$ and $v$, adequately explains the increase in $R$ above $F R C$, but does not explain the increase in $R$ below FRC. This suggests that an additional mechanism(s) is responsible for increasing $R$ helow FRC. Supported by NHLBI HL19298 and Dept. of Veterans Affairs.

16

\section{TISSUE STRETCH AND PULMONARY INTERSTITIAL PRESSURE Matthew R. Glucksberg Northwestern University, Evanston, IL 60208}

Interstitial pressure, an important parameter in transvascular fluid transport and lung liquid balance, has long been known to reflect the external stresses acting on the interstitium as well as its internal structure. In many tissues the hydrostatic pressure normally hovers near ambient atmospheric pressure, however in the lung, where stress distributions are complex and include both compressive stress due to ainway pressure and tissue tension due to the transpulmonary pressure, interstitial pressures may differ from ambient pressures. To show the effect of tissue tension on interstitial pressure I report micropuncture servonull measurements of pressures in the interstitium surrounding the vessels of the hilum subjected to stretch. In 5 excised lobes of cat lungs, I cannulated the pulmonary artery, vein and airway, pertused the lobe with blood and connected the airway to a constant pressure $\left(7 \mathrm{cmH}_{2} \mathrm{O}\right)$ air source. The cannulae were tied together and attached to an apparatus that was used to apply a known distension to the hilar region. A video system was used to estimate strains from dye marks on the pulmonary artery and vein. At zero stretch hilar interstitial pressure was $3 \pm 2 \mathrm{cmH}_{2} \mathrm{O}$, which decreased to $-9 \pm 3 \mathrm{cmH}_{2} \mathrm{O}$ when the distance between the dye marks reached $130 \%$ of the initial (zero stretch) distance. I conclude that tensile stress contributes to a lower interstitial pressure and should be considered an important determinant of interstitial pressure in the lung. Supported by a grant from The Whitaker Foundation.

17

PULMONARY CIRCULATION - CORRELATION OF THEORY AND EXPERIMENTS

R. Z. Gan, *M. A. Olman and R. T. Yen

Department of Biomedical Engineering, Memphis State University, Memphis, TN 38152

*Deparment of Medicine, University of Alabama at Birmingham, Birmingham, AL 35294

The characteristics of pulmonary circulation is presented as the measurements of pulmonary blood pressure and flow rate in many experimental investigations. The analysis of these pressure and flow measurements must be performed with a theoretical model so that a better understanding of the physiology of blood circulation in the lung can be obtained. In this paper, we are providing a hemodynamic model based on the directly measured anatomic sinucture and mechanical properties of the dog pulmonary vasculature and, then, validating the model with the animal experimental observations. The model consists of 12 orders of arteries (excluding the main pulmonary artery) observations. The model consists of 12 orders of arteries (excluding the main pulmonary artery)
and 11 orders of veins. The order is distinguished by the diameter in Strahler's system and and 11 orders of veins. The order is distinguished by the diameter in Strahler's system and elastic tubes with the measured average diameter, length, and compliance of the vessel wall. The elastic tubes with the measured average diameter, length, and compliance of the vessel wall. The
arterial and venous trees converge at the capillary sheel where the vascular space between the arterioles and venules is formed by two alveolar-capillary membranes with a set of regularly spaced "posts". The "sheet-flow" theory is used to describe the blood flow in capillaries. Under the given airway pressure, pleural pressure, and cardiac output, the pulmonary pressure-flow relationship and the pressure distribution are derived. It shows that the positive and negative inflations have different effects on the blood flow in the lung. The nonlinearity of pressure-flow curve and the resistance of pulmonary circulation system are related to the elasticity of blood vessels and the Starling resistors. In the mean time, the experimental observations of pulmonary arterial pressure-flow relations in normal and chronic pulmonary thromboembolic dogs are completed and compared with the model-derived results. The comparisons show good agreement between the experiments and theoretical modeling. Finally, the model is used to predict the effect of degrees of central pulmonary obstruction on pulmonary circulation. 
18

NEUTROPHIL MARGINATION IN THE PULMONARY MICROVASCULATURE. C.M. Doerschuk, B.R. Wiggs, W.M. Quinlan, N.A. Doyle, and J.C. Hogg. H.B. Wells Center for Pediatric Research, Section of Pulmonology, Department of Pediatrics, Indiana University.

The pulmonary microvasculature contains an increased concentration of neutrophils compared to blood in large vessels due to the longer capillary transit time of neutrophils compared to RBC. To investigate the mechanisms underlying this difference in transit, measurements of the diameter of capillary segments and the spherical diameter of neutrophils were compared and showed that neutrophils must deform to pass through $60 \%$ of the segments. Comparison of the extractions of neutrophils and nondeformable microspheres measuring 3.1, 4.6, or $5.8 \mu \mathrm{m}$ in diameter on a single transit through the pulmonary microvasculature showed that neutrophils could deform as rapidly as RBC from a spherical diameter of $6.4 \mu \mathrm{m}$ to approximately $4.8 \mu \mathrm{m}$, but further deformations required longer times. The minimum diameter of the perfused capillary pathways was about $4.4 \mu \mathrm{m}$ and was narrower in regions of the lungs where margination was increased. This pathway diameter was larger than predicted based upon a random distribution of capillary segments within capillary pathways, suggesting that wider segments were preferentially perfused. These data suggest that margination of neutrophils is due to differences in deformability of neutrophils and RBC and the anatomy of the pulmonary capillary bed which provides multiple parallel pathways for the rapidly moving RBC to bypass temporarily obstructing neutrophils.

19

DEVELOPMENT OF A CONSTANT PERFUSION VASCULAR-TISSUE MODEL OF PMN-MEDIATED LUNG PERMEABILITY INJURY. F.R. Haselton, J. S. Alexander, and J. H. Woodall. Vanderbilt University, Department of Biomedical Engineering.

A principal defect in the vascular injury associated with the entrapment of activated neutrophils (PMN) is increased microvascular permeability $(P)$. To investigate the role of activated PMN in increased endothelial monolayer $P$, we perfused our previcusly described cell-column model of the vasculature with $2 \times 10^{6} \mathrm{PMN} / \mathrm{ml}$ at a local average velocity and pressure of $0.09 \mathrm{~cm} / \mathrm{sec}$ and $5-45 \mathrm{~mm} \mathrm{Hg}$. Shear stress was estimated to be 0.1 to of endothelial surface in 15 mins. This rate was significantly greater than without endothelium. Circulating unactivated PMN for 60 min did not increase endothelial $P$ and did not activate PMN. fMLP $\left(10^{-5} \mathrm{M}\right)$ selectively activated neutrophils recirculating through cell-columns and compared to PMN alone significantly increased the rate of loss of circulating PMN in the cell-column (25\%). This reduction of circulating PMN with IMLP was not affected by addition of superoxide dismulase (SOD, $142 \mathrm{U} / \mathrm{ml}$ ), catalase (CAT, $1398 \mathrm{U} / \mathrm{ml})$, the elastase inhibitor $\left(L 658,75810^{-5} \mathrm{M}\right)$ or a combination of SOD and CAT. Endothelial monolayer $P$ was not affected by fMLP or by PMN + fMLP, but was significantly elevated to $2.99 \times$ baseline by a $30 \mathrm{~min}$ treatment with PMN + fMLP + SOD. This increase in $P$ was significantly attenuated (1.27 $X$ baseline) by the addition of CAT. Neither superoxide (xanthine oxidase at $0.25,7.5,75 \mathrm{mU} / \mathrm{ml}), \mathrm{H}_{2} \mathrm{O}_{2}\left(10^{-5} \mathrm{M}\right)$ or elastase alone $(33.2,332 \mathrm{mU} / \mathrm{ml})$ affected $P$ in 60 mins, but $P$ was significantly increased by a $30 \mathrm{~min}$ treatment with $10^{-3} \mathrm{M} \mathrm{H}_{2} \mathrm{O}_{2}(1.74 \mathrm{X}$ baseline). These data suggest that the increased $P$ which occurs with a constant perfusion of $P M N$ activated with $\mathrm{fMLP}$ in the presence of $\mathrm{SOD}$ may involve $\mathrm{H}_{2} \mathrm{O}_{2}$, but that $\mathrm{H}_{2} \mathrm{O}_{2}$ by itself is not necessarily the direct cause of the observed $P$ increase. (In part by NIH 40554).

20

OPTICAL MEASUREMENT OF LUNG FILTRATION COEFFICIENT $\left(\mathrm{K}_{\mathrm{fc}}\right)$ USING POLYSULFONE FIBERS. J.W. Klaesner, R.J. Roselli, R.E. Parker, N.A. Pou, and M.E. Parham. Department of Biomedical Engineering and Pulmonary Circulation Center, Vanderbilt University, Nashville, TN 37235, and W.R. Grace \& Co. - Conn., Lexington, MA 02173.

We measured lung filtration coefficients $\left(\boldsymbol{K}_{\mathbf{f c}}\right)$ using optical methods that correspond to the gravitational method corrected for blood volume increases (J. Appl. Physiol. 73(6):2649, 1992) The gravimetric method requires an isolated lung preparation and continuous measurement of weight changes in order to calculate the $\mathrm{K}_{\mathrm{fc}}$, whereas the optical method requires protein concentration measurements following a step change in pressure. A major drawback of this optical measurement has been the necessity for low hematocrit perfusates because the optical method is obscured by the absorbence of red blood cells. Even with corrections for smal changes in perfusate hematocrits, only pressure elevations provided data that could be used to calculale $K_{\mathrm{fc}}$. Absorbance measurements during pressure decreases were too noisy to discern changes in protein concentrations. The development of polysulfone fibers (W.R. Grace \& $\mathrm{Co}$.) has provided on-line separation of red blood cells from plasma thus eliminating this restriction and allowing for optical measurements to be made at physiological hematocrits and flows. The $\mathrm{K}_{\mathrm{fc}}$ was then calculated from both increases and decreases in venous pressures. In one such experiment, the $\mathrm{K}_{\mathrm{fc}}$ 's measured were 0.413 for a pressure elevation of $20 \mathrm{cmH}_{2} \mathrm{O}$, and 0.367 for a similar pressure decrease, which were smaller than the gravimetric computation of 0.502 $\left(\mathrm{ml} / \mathrm{min} / \mathrm{cmH}_{2} 0 / 100 \mathrm{gDLW}\right)$. Thus we conclude that optical measurements provide an accurate assesment of lung filtration coefficients, and allow for measurement of $\mathrm{K}_{\mathrm{fc}}$ in intact animals, which is not possible with the gravimetric method. Eventually we expect that this method, with refinement and miniaturization, will allow for measurement of $\mathrm{K}_{\mathrm{fc}}$ in humans. (Supported by HL 41129 and HL 07411)
21

THE USE OF FITC-DEXTRANS TO CHARACTERIZE LUNG VASCULAR PERMEABILITY. R. J. Roselli, V. J. Abernathy, R. E. Parker, N. A. Pou. Department of Biomedical Engineering and Pulmonary Circulation Center, Vanderbilt University, Nashville, TN 37235.

Lung microvascular permeability was examined by comparing the transvascular transport of polydisperse FITC-dextrans with the transport of endogenous proteins in unanesthetized sheep having a lung lymph fistula. We infused 2.5 $\mathrm{g}$ of FITC-dextrans between $30 \mathrm{kDa}$ and $2000 \mathrm{kDa}$ over a period of 25 hours, collecting plasma and lymph samples periodically. During the last three hours we also infused $0.67 \mathrm{~g}$ of small molecular weight FITC-dextrans (mean molecular weight of $9 \mathrm{kDa}$ ). Plasma and lung lymph samples collected after 25 hours were separated according to Stokes-Einstein radii by passing them through a Sephacryl- 300 column. The resulting FITC-dextran fractions $(0.5 \mathrm{~nm}$ $9 \mathrm{~nm}$ ) were compared with endogenous proteins separated using gradient pore gel electrophoresis $(3.6 \mathrm{~nm}-9.6 \mathrm{~nm})$. Results indicate that essentially $100 \%$ of FITC-dextrans smaller than $1.2 \mathrm{~nm}$ can freely pass through the lung microvascular barrier, while less than $10 \%$ of FITC-dextrans larger than $4 \mathrm{~nm}$ normally cross the barrier. Significant FITC-dextran sieving occurs in the range between $1.2 \mathrm{~nm}$ and $4 \mathrm{~nm}$, similar to that reported for neutral dextrans by Lanken et al. (J. Appl. Physiol. 59:580, 1985). Proteins of the same size, however, pass through the barrier much more easily, suggesting that FITCdextran transport is more restrictive than protein transport, even though both species are negatively charged. (Supported by HL19153)

\section{2}

MODEL OF ANGIOTENSIN CONVERTING ENZYME KINETICS De La Fuente, K.D., M.P. Merker, C.A. Dawson, J.H. Linehan Marquette University; Medical College of Wisconsin and VAMC, Mitwaukee, WI 53217

Pulmonary angiotensin converting enzyme (ACE) hydrolyzes substrates which contain proline or proline derivatives. Rotation about the proline-peptide bond allows interconversion between cis and trans conformers. Existing models of cis-trans substrate-enzyme hydrolysis kinetics assume only trans is hydrolyzed. Trans-only models have an empirical basis in kinetic studies involving single-pass lung perfusion wherefore a constant fraction of tracer amounts of substrate remains unhydrolyzed regardless of flow rate. This fraction is nearly equal to the cis fraction of the substrate suggesting that only trans is hydrolyzed. Saturating dose trans-only models do no completely explain the data (Linehan, et al., Am. J. Physiol. 257,2006-16, 1989). To this end, the following chemical reactions were hypothesized.

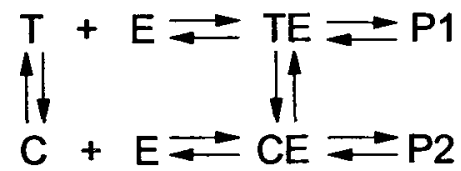

where $T$ and $C$ are the trans and cis conformers, $E$ is $A C E, T E$ and $C E$ are the enzymesubstrate complexes, and $\mathrm{P} 1$ and $\mathrm{P} 2$ are the metabolites. These chemical reactions and the convective transport of substrate with blood were included in a capillary model. Simulation studies were directed toward identifying which kinetic processes dominate the fate of substrate on a single passage through the lung. Supported by the Veterans Administration and by National Institutes of Health $\mathrm{HL} 24349$. 
24

TRANSITION TO TURBULENCE IN OSCILLATING FLOWS THROUGH A MODEL BRONCHIAL BIFURCATION R.E. Popwell and R.A. Peattie

Dept. of Biomedical Engineering, Tulane University, New Orleans, LA 70118

Conditions necessary to induce transition to turbulence in oscillating flows have been studied in a rigid model of a bronchial bifurcation. The model, which was cast in a transparent silicone elastomer, replicated the geometry of a human bronchial bifurcation, as determined from literature and observations of CT scans. Experimental flows having tidal volumes of 317,32 , and $26 \mathrm{ml}$ and frequencies ranging from 0.01 to $1.0 \mathrm{~Hz}$ were driven through the model by a piston-cylinder apparatus. These flows generated Reynolds numbers, $R e$, between 400 and 5000, and Womersley numbers, $\alpha$, between 2 and 24 , providing a dynamic match to in vive tracheal air flows for conditions ranging from normal resting breathing to high frequency ventilation. Frequency shifted, dual-beam, laser-Doppler velocimetry was used to measure the instantaneous local velocity field. Fully laminar flow was observed for $R e<600$ and $\alpha<2.75$. For values of $R e>600$ and $\alpha>2.75$, however, turbulent-like bursts were observed in the deceleration phase of the expiratory part of the flow cycle. Our continuing investigations extend these results over a wide range of Re and $\alpha$.

Acknowledgments - The authors gratefully acknowledge the support of the Louisiana Educational Quality Support Fund

25

QUANTIFIED FLOW VISUALIZATION OF RESPIRATORY FLOWS: MODEI STUDIES. E.H. Fefferman, J.C. Bennet, and M.A.F. Epstein, Universizy of Connecticut, Storrs, Connecticut, 06269-3139.

Laser Induced Fluorescence (LIF) techniques are being developed for quantification of mass flux distribution in scaled mechanical models of airway bifurcations. The glass models studied were two single bifurcations with an included angle of seventy $(70)$ degrees and total cross-sectional area ratios for daughter branches to mother branches of $100 \%$ and $120 \%$. Preliminary studies were performed in a straight tube. Initial testing was done using conventional flow visualization, using planar laser illumination and conventional video camera image recording. These results allowed selection of bifurcation regions for quantification of dye concentration using point-wise laser illumination and photomultiplier (PM) signal acquisition.

With water as the working fluid, the Reynolds numbers used were about 500 for steady inspiratory flows. Fluorescein dye was injected at a known location well upstream of the bifurcation. The dye concentration was sufficiently low to linearly relate the PM output voltage and the dye concentration.

The presentation will discuss the test procedure, documentation of the repeatability of the calibration and test procedures, and sample results for dye concentration contours. These results will be useful in evaluation of tlow prediction models concerned with the distribution of gases and aerosols introduced into the airways during normal and mechanically assisted ventilation.

26

HARMONIC DISTORTION FROM BROADBAND SIGNALS ASSOCIATED WITH NONLINEAR LUNG MECHANICS DURING BRONCHOCONSTRICTION IN DOGS. Q. Zhang, B. Suki, and X.R. Lutchen.

Department of Biomedical Engineering, Boston University, Boston. MA

We have developed a class of pseudo-random waveforms which avoids harmonic distortion and cross-talk at the input frequencies and allows us to track the frequency and tidal volume (VT) dependence of lung resistance (R) and Elastance (E) from $0.2-5 \mathrm{~Hz}$. We found that lung mechanics can be influenced by tissue and airflow nonlinearities. The harmonic interactions in response to external forcing should be characteristic of these Donlinearities. In this study we developed a simple index to quantify the harmonic distortion in our broadband signal. Data was analyzed from 4 dogs forced at VT of $100 \mathrm{mll}$ and $300 \mathrm{ml}$ before and during constant infusion of histanine. We used a computer driven waveform delivered to an oscillator which ideally results in a flow input containing energy only at specific frequencies. Our index of harmonic distortion $\left(k_{\imath}\right)$ represented the percent energy in the output pressure at frequencies where energy was not purposely placed in the input. In practice, additional flow energy occurs at other frequencies. We compensated for linear transformation of this spurious energy by first fitting the impedance spectra at a given VT with a linear model and then using this model to analytically compute the energy transfer due to the linear subsystem. This index was applied during control and peak constriction to the data from each VT. During control we found a small but significant VT dependence in $R$ and $E(p<.01)$ and a corresponding $k_{d}$ of $5 \%$ with $100 \mathrm{ml}$ increasing to $7-11 \%$ at $300 \mathrm{ml}$. During peak response, the VT dependence increased in both severe and milder responding dogs. For a severe responder the $k_{f}$ increased by $23.5 \%$ with a $100 \mathrm{ml}$ VT and $25.8 \%$ with a $300 \mathrm{ml}$ VT. In a milder responder the $\mathrm{k}_{4}$ increased only for $300 \mathrm{ml}$ VT $(12.9 \%)$. These results suggest that indices of harmonic distortion provide additional information on the presence of nonlinearities and may be useful when measurements at more than one VT are not possible. Supported by NSF BCS-9011168.
27

EXPERIMENTAL MODEL OF THE OPENING OF A COLLAPSED AIRWAY Matthew L. Perun and Donald P. Gaver

Dept. of Biomedical Eng., Tulane University, New Orleans, Louisiana, 70118

Airway collapse occurs in the elderly, emphysematics and premature neonates and in severe cases impairs gas exchange. We designed a benchtop model consisting of a collapsed polyethylene tube with a Newtonian fluid that acted as a viscous adhesive. Downstream of the air-liquid interface the tube was collapsed in a ribbon-like manner, while upstream of the interface the tube had a rectangular cross-section due to the constraints of the Plexi-glas sheets. This procedure modified the upstream shape from the circular cross-section observed in unconstrained tube, thus simplifying the reopening geometry. 'We examined how the fluid properties (viscosity, $\mu$, surface tension, $\gamma$, and density, $\rho$ ) and the structural characteristics of our model (effective diameter, $D$, longitudinal tension, $T$, and fluid film thickness, $H$ ) affected the opening of the tube. We measured the pressure $(P)$ required to move the meniscus down the tube at a constant velocity (U), and found the dimensionless post-startup pressure $(\mathrm{PD} / \gamma)$ increased monotonically with the capillary number $(\mathrm{Ca}=\mu \mathrm{U} / \gamma)$, and steadystate existed when $\mathrm{Ca}>0.6$. D was the most dominant structural characteristic, which caused an increase in the post-startup pressure (P) for a decrease in D. A ninefold increase in $\mathrm{H}$ only caused a slight decrease in the reopening pressure. However, spatial variation in $\mathrm{H}$ resulted in a transient increase in pressure. A sixfold change in $T$ did not significantly affect the reopening pressure. We also investigated the startup behavior and found that the these pressures depended upon the fluid viscosity and piston acceleration. Low viscosities and large accelerations caused peak startup pressures that were greater than the average post-startup pressure. Based on our results, we predicted airway reopening times and pressures for the respiratory bronchioles

This research was funded by the Whitaker Foundation and the National Science Foundation Grant BCS9209558. DPG is a Parker B. Francis Fellow in Pulmonary Research

28

\author{
PULMONARY AIRWAY REOPENING: A NUMERICAL STUDY \\ OF THE INFLUENCE OF SOLUBLE SURFACTANT \\ Vithal R Tekumalla and Donald P Gaver \\ Dept. of Biomedical Eng., Tulane University, New Orleans, LA 70118
}

In the reopening of a closed pulmonary airway, the presence of soluble surfactants play a significant role in altering the reopening mechanisms. The reopening process is modeled as the motion of a long bubble in a Hele-Shaw cell under the influence of single constituen soluble surfactant. A computational model is developed that incorporates: a) bubble hydrodynamics b) bulk surfactant transport c) stress conditions at the interface d) surfactan flux exchange between the bulk and air-liquid interface and e) interaction between the hydrodynamics and physicochernical aspects of the system. The model uses a composite-mesh based finite-difference technique. The solution to the problem is obtained as a function of the non-dimensional parameters, which are the capillary number $(\mathrm{Ca}=\mu \mathrm{U} / \sigma)$, Peclet number $(\mathrm{Pe}=\mathrm{Ub} / \mathrm{D})$, Marangoni number $(\mathrm{Ma}=-\mathrm{Cb} / \sigma(\partial \sigma / \partial \Gamma))$, Stanton number $(\mathrm{St}=\mathrm{k} / \mathrm{U})$ and partition coefficient $(\mathrm{K})$. Here $U$ is the bubble velocity, $b$ is the tube thickness, $\mu$ is bulk phase viscosity, $D$ is bulk diffusivity, $\sigma$ is surface tension, $k$ is the interfacial adsorption coefficient, $\mathrm{K}$ denotes the relationship between bulk and interfacial concentrations a equilibrium and $\mathrm{C}$ is the upstream uniform bulk concentration. Results of an extensive parametric study are presented depicting their influence on bubble shape and pressure drop across the interface and their physiological significance will be discussed.

This research was funded by the Whitaker Foundation and the National Science Foundation BCS9209558. DPG is a Parker B. Francis Fellow in Pulmonary Research.

\section{9}

TIME- VS. FREOUENCY-DOMAIN ANALYSIS OF RESPIRATORY MECHANICS AT LOW FREQUENCIES: IMPACT OF WAVEFORM

D.W. Kaczka, B. Suki, and K.R. Lutchen

Department of Biomedical Engineering, Boston University, Boston, MA 02215

A potential approach to determine frequency dependence in respiratory resistance $\left(R_{n}\right)$ and elastance $\left(E_{m}\right)$ is to use a multi-frequency (broad-band) ventilator forcing signal optimized to deliver a volume sufficient to maintain gas exchange. We compared frequency-domain and time-domain techniques to estimate respiratory resistance $\left(\boldsymbol{R}_{n}\right)$ and elastance $\left(\boldsymbol{E}_{n}\right)$ at low frequencies $(0-3 \mathrm{~Hz})$ in humans ventilated with these waveforms. The influence of system nonlinearities on the spectral estimate may depend on the lechnique employed. The frequencydomain approach utilizes the Fast Fourier Transform (FFT) on sampled airway pressure and flow data, while the time-domain approach uses multiple linear regression to estimate specific mechanical parameters in a two-compartment transfer function model. We used two forms of optimal ventilator waveforms (OVW's) with improved signal-to-noise ratio. One OVW contained harmonics that were integer multiples of each other (IM), while the other had contained harmonics that were integer multiples of each other (IM), while the other had
harmonics selected to minimize nonlinear harmonic distortions and cross-talk (NSND). We evaluated $\boldsymbol{R}_{n}$ and $\boldsymbol{E}_{n}$ spectra in six healthy human volunteers at tidal volumes of 350 and 650 $\mathrm{ml}$. For the time-domain approach, parameter estimates obtained using IM-OVW signals were much more sensible at any given $V_{T}$ compared to estimates using waveforms optimized to avoid barmonic distortions. FFT-based spectral estimates of $\boldsymbol{R}_{n}$ and $\boldsymbol{E}_{\mathrm{g}}$ using IM-OVW's were erratic and inconsistent, while NSND-OVW's provide smooth, reliable estimates of $R_{n}$ and $E_{n}$ out to $3 \mathrm{~Hz}$. The experimental results were consistent with simulation studies using a nonlinear model of the respiratory system. We conclude that time-domain identification is more tolerant of harmonic distortions for an IM-OVW input. Conversely, the NSND-OVW is more appropriate for probing frequency dependence in $R_{n}$ and $E_{n}$. (supported by NSF BCS9011168) 
30

ELECTRON PROBE MICROANALYSIS OF RAT BRAIN CORTEX IN RESPIRATORY ACIDOSIS AND ANION TRANSPORT INHIBITION. RS Schacterle, RA Craig, TE Hutchinson, and JM Adams, Biomedical Engineering, University of Virginia, Charlottesville, VA 22908

We investigated the effects of acute hypercapnia during a rebreathing maneuver, and hypercapnia following anion transport inhibition by DIDS, on cortical intracellular element concentrations in anesthetized rats. Frozen tissue samples of the parietal cortex were obtained, and intracellular dry weight concentrations were measured from thin sections of these samples using electron probe microanalysis. This allowed for simultaneous measurement of $\mathrm{Na}, \mathrm{Mg}, \mathrm{P}, \mathrm{S}, \mathrm{Cl}, \mathrm{K}$, and $\mathrm{Ca}$. Concentrations in cell water and intracellular $\mathrm{pH}$ were calculated from the measured dry weight values. Increased $\mathrm{CO}_{2}$ levels caused a significant decrease in intraceliular $\mathrm{Mg}, \mathrm{P}$ and $\mathrm{Cl}$ concentration in mmol/kg dry weight compared to sham rats. No significant changes in $\mathrm{Na}, \mathrm{S}, \mathrm{K}$ or $\mathrm{Ca}$ dry weight concentrations were detected. Changes associated with hypercapnia following DIDS application were decreases in $\mathrm{Mg}, \mathrm{P}, \mathrm{S}$ and $\mathrm{K}$ and a restoration of $\mathrm{Cl}$ back to sham values; $\mathrm{Na}$ and $\mathrm{Ca}$ did not change. Calculated concentrations per $\mathrm{Kg}$ of $\mathrm{H}_{2} \mathrm{O}$ showed that $\mathrm{Na}, \mathrm{Cl}$ and $\mathrm{K}$ did not change with hypercapnia, and that $\mathrm{Cl}$ increased following DIDS and hypercapnia. Calculated intracellular $\mathrm{pH}$ was 6.98 in the sham rats, was 7.13 in acute hypercapnia, not a significant change, but decreased to 6.46 following DIDS. These data show that cellular ion changes in acute hypercapnia act to maintain intracellular $\mathrm{pH}$ in the face of rising $\mathrm{PCO}_{2}$, and that DIDS prevents this response leading instead to cellular acidification. Supported by NIH HL29396

31

DISTRIBUTION OF OZONE ABSORPTION IN INTACT HUMAN LUNGS Asplund PA, Ben-Jebria A and Uitman JS

Pennsylvania State University, University Park, PA

To assess the health effects of ozone $\left(\mathrm{O}_{3}\right)$ inhalation, the sites of absorption must be known. In the bolus-response method developed in our laboratory, a subject breathe through a mouthpiece assembly from which a $20 \mathrm{ml}$ bolus of $\mathrm{O}_{3}$ is injected into the inspired airstream. Respiratory airflow and $\mathrm{O}_{3}$ concentration are monitored near the lips, and the fraction of absorbed $\mathrm{O}_{3}$ is computed from the integrals under the inspired and expired $\mathrm{O}_{3}$ concentration curves. By injecting boluses at a variety of times during inspiration, the absorbed fraction is determined as a function of penetration volume into the respiratory tract. Our first study was conducted on a group of 9 healthy young men who were previously unexposed to $\mathrm{O}_{3}$. During quiet breathing at a respiratory flow of 250 $\mathrm{ml} / \mathrm{sec}, 50 \%$ of inhaled $\mathrm{O}_{3}$ was absorbed in the first $70 \mathrm{ml}$ of airway penetration (i.e., the upper airways) while the remainder was absorbed at $180 \mathrm{ml}$ of airway penetration (i.e., the terminal bronchioles). To measure the $\mathrm{O}_{3}$ absorption distribution under more realistic conditions, experiments are now being conducted on a group of subjects after they are exposed to a constant $\mathrm{O}_{3}$ level of $0.1-0.3 \mathrm{ppm}$ for 2 hours (Supported in part by NIEHS Grant ES06075)
32

SPECTRAL MEASURES FOR IDENTIFYING CHANGES IN AIRWAY GEOMETRY IN VIVO FROM REFLECTED SOUND DATA. MAF Epstein, PL Conard, AB Smith, F Moslehi. Anesthesiology Research Laboratory, University of Connecticut Medical School, Farmington, CT.

Previous work in our laboratories using mechanical lung models have demonstrated that blockage of a major bronchus changes the distribution of power in the reflected sound spectrum. In this study, a continuous white noise input $(2 \mathrm{~Hz}-5000 \mathrm{~Hz})$ was introduced into the endotracheal tube in nine supine, anesthetized, intubated, spontaneously breathing dogs. Replicate measurements of the reflected sound were made within a $50 \mathrm{msec}$ period at end exhalation (when airflow was negligible) for normal lung conditions and when either the left or right mainstem bronchus was blocked for a short time lless than three minutes) by inflation of the balloon at the end of a fogarty catheter. The reflected sound data were acquired with a PC-based system and stored for analysis. The spectra for the normal lung condition for each dog were ensernble averaged and normalized by the total power of the absolute ensemble averaged spectrum. The same procedure was used to process the data for the blocked lung condition. The differences between the normalized average spectra for normal and blocked conditions were calculated for each dog, and the cumulative difference was plotted as a function of frequency. In seven of the nine dogs, the normalized cumulative difference was positive for frequencies below $2500 \mathrm{~Hz}$, indicating shift of power from lower to higher frequencies due to the blocked conditions. These results indicate that measurement of reflected sound may provide a rapid, noninvasive method for detecting large changes in airway geometry. 
33

BODY SURFACE LAPLACIAN ELECTROCARDIOGRAPHIC IMAGING Bin He and Richard J. Cohen

Harvard University - Mass. Inst. of Tech. Division of Health Sciences and Technology

Cardiac electrical activity is distributed over the three dimensional volume of the myocardium. The ability to noninvasively map regional myocardial events will greatly enhance our capability to better understand cardiac electrophysiological processes and to diagnose cardiovascular disease. We have developed a novel technique-body surface Laplacian electrocardiographic imaging to resolve distributed cardiac electrical activity with high spatial resolution. We have developed a bipolar electrode array and high resolution imaging system to directly measure and visualize the body surface distribution of the surface Laplacian of the electrical potential. Computer simulations and physical tank studies were conducted to elucidate the behaviors of the body surface Laplacian images (BSLIs) as compared with body surface potential maps (BSPMs). The ability of body surface Laplacian imaging has been further tested in intact animals where BSLIs and BSPMs were measured and compared with the known sources during epicardial pacing. We have also investigated BSLIs in normal and abnormal human subjects. Our work to date indicates that the BSLI can provide a high resolution two dimensional projection image of actual three dimensional cardiac electrical activity, and that the BSLI has greater spatial resolution than conventional BSPM in resolving multiple spatially separated myocardial events. Body surface Laplacian electrocardiographic imaging promises to be a powerful tool for cardiac electrophysiology research and clinical diagnosis of cardiovascular disease.

\section{4}

COMPUTER SIMULATIONS OF ACTION POTENTIAL PROPAGATION IN A THREE-DIMENSIONAL HISTOLOGICALLY BASED MODEL OF VENTRICULAR MYOCARDIUM

A.E. Pollard

Department of Biomedical Engineering, Tulane University, New Orleans, Louisiana

Abstract: Three-dimensional simulations of action potential propagation in a set of models with features intended to closely represent the histology of ventricular myocardium were performed. Serial sections from animal preparations were acquired at $500 \mu \mathrm{m}$ intervals in sections of the canine pulmonary conus. Within each serial section, fiber directions and the positions for large resistive inhomogeneities (fat and blood vessels) were identified. This information was then used in the assembly of meshes which were buitt from microscopic elements arranged in rectangular parallelepipeds. For the simulations, the system of membrane ionic currents responsible for action potential initiation and maintenance was described with the nonlinear Ebihara and Johnson membrane equations for the fast sodium current. Conductivities in the models were assigned to represent intramural rotation of the fiber axes for both abrupt and gradual rotation. Epicardial stimuli were applied for each simulation. Three-dimensional activation patterns and epicardial isochrone maps from the simulations were compared with maps generated from experimental recordings. A substantive influence of the ventricular conduction system on the activation patterns observed on the epicardial surface was documented.

Acknowledgements: The Whitaker Foundation. The Louisiana Educational Quality Support Fund. U.S. Public Health Service Grant RO1 HL34288. The Richard A. and Nora Eccles Harrison Fund for Cardiovascular Research and the Nora Eccles Tread well Foundation.

35

\section{APPLICATION OF A MATHEMATICAL MODEL TO STUDY CONDUCTION THROUGH AN EXTRACELLULAR POTASSIUM GRADIENT IN A MODELED CARDIAC FIBER \\ B J Muller-Borer, T A Johnson, L S Gettes, W E Cascio}

Ionic and resistive changes contributing to unidirectional block were studied Ionic and resistive changes contributing to unidirectional block were studied
using a computer model of a one dimensional cardiac fiber. A detailed ionic current-based mathematical model of the ventricular cardiac action potential, proposed by Luo and Rudy, was implemented in a discrete electrical network. The modeled cardiac fiber was composed of discrete cellular elements, resistively coupled to form random sized modeled cells $(30 \mu \mathrm{m}$ to $130 \mu \mathrm{m}$ in length) The modeled cells were coupled through a gap junction resistor $\left(r_{\text {gap }}\right.$ ) to form a $13 \mathrm{~mm}$ long fiber with an extracellular $\mathrm{K}^{+}$concentration $\left(\left[\mathrm{K}^{+}\right]_{\mathrm{o}}\right)$ gradient $(4 \mathrm{mM}$ to $12 \mathrm{mM}$ ) along its length. Under well-coupled conditions an action potential propagated through the modeled fiber when a current stimulus was applied both at the low and high $\left[\mathrm{K}^{+}\right]_{0}$ end. Changes in the resting membrane potential and conduction velocity were recorded as the signal propagated through the fiber and $\left[\mathrm{K}^{+}\right]$gradient. A high resistive barrier, $1 \mathrm{~mm}$ in length, was introduced in the mid- $\left[K^{+}\right]_{0}$ region $(8 \mathrm{mM})$ by increasing $r_{\text {gap }}$ in this region by $5,7.5$ and 10 times normal. Action potentials propagated along the fiber and through the resistive barrier with increases in $\mathrm{I}_{\text {gap }}$ when stimulated at the low $\left[\mathrm{K}^{+}\right]_{0}$ end When stimulated at the high $\left[\mathrm{K}^{+}\right]_{0}$ end, action potentials propagated through the fiber as $I_{\text {gap }}$ in the resistive barrier was increased by $5 x$ and $7.5 x$, but blocked when $r_{\text {gap }}$ was increased by $10 \mathrm{x}$. These simulations suggest that conduction leading to block within a $\left[\mathrm{K}^{+}\right]_{0}$ gradient is not only sensitive to the degree of cellular uncoupling, but also dependent on the direction of propagation.
36

FLEXIBLE, POLYIMIDE-BASED MICROELECTRODE PLAQUE DESIGN FOR IONIC AND ELFCTRICAL CHARACTERIZATION OF ISCHEMIC HEARTS BE Billard, TA Johnson, RB Ash, HT Nagle, E Lindner, VV Cosofret, and RP Buck, Experimental Cardiology Group, University of North Carolina at Chapel Hill

Regional myocardial ischemia, electrically and jonically manifest as altered activation sequence, slowed conduction velocity $(\mathrm{CV})$ and disrupted transmembrane cation gradients, promotes the formation of reentry circuits leading to cardiac arrhythmias and ventricular fibrillation (VF) Technical limitations of ionic and electrical measurements have been surmounted using unique, easily modified, 2D, flexible, polyimide-based microelectrode plaque designs. The current plaque has 144 non-polarizable $\mathrm{Au} / \mathrm{Ag} / \mathrm{AgCl}$ electrical

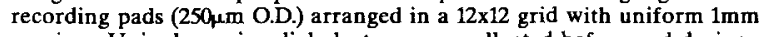
spacing. Unipolar, epicardial electrograms collected before and during acute, regional ischemia in in situ pig hearts demonstrate high amplitude, low drift and good SNR using standard laboratory amplification and filtering techniques $(50 \mathrm{X}, 0-500 \mathrm{~Hz} @-1 \mathrm{~dB})$. Simultaneous unipolar and bipolar digital recordings $(2 \mathrm{KHz} / \mathrm{site})$ were used to verify local activation time as $-d \mathrm{~V} / d \mathrm{t}_{\text {max }}$ in the unipolar electrogram and confirm the accuracy of bipolar signal generation by adjacent unipolar subtraction. Computational algorithms have been developed and implemented that permit beat-to-beat animation of raw data using AVSTM software and statistical quantification of the activation wavefront using spectral analysis. Calculations of epicardial longitudinal CV using these plaque designs show that CV falls from a control of $50 \mathrm{~cm} / \mathrm{sec}$ to $20-25 \mathrm{~cm} / \mathrm{sec}$ after $10 \mathrm{~min}$ of no-flow ischemia. Finally, the deposition of ion-selective membranes on the polyimide substrate for extracellular $\mathrm{K}^{+}$(valinomycin) and $\mathrm{H}^{+}$(TDDA) measurement and their adaptability for transmural implantation permits the disclosure of ionic and electrical correlates essential to ischemia-induced VF.

37

THE PROTOTYPE OF A KNOWLEDGE BASED SYSTEM FOR MONITORING AND CLASSIFICATION OF ECG SIGNALS

K.J. Siddiqui, E.C. Greco, N.N. Kadri, S. Mohiuddin, M.H. Sketch

The Cardiac Center of Creighton University

A bottleneck in the process of building the knowledge base of an intelligent system is the retrieval of the appropriate problem-solving knowledge from the human expert. The expert knowledge is usually available in highly unstructured form which is often difficult to transform in computer readable form. Some of these difficulties can be resolved by using decisiontheoretic methods. These methods can be developed using primitive knowledge components theoretic methods. These methods can be developed using primitive knowledge components
extracted from physical observations. Methods of knowledge acquisition and representation extracted from physical observations. Methods of knowledge acquisition and representation
from the fields of signal processing, pattern recognition and artificial intelligence can be used to acquire the pertinent knowledge components and then to use them to determine the to acquire the pertinent knowledge components and then to use them to determine the
characteristics of the individual pattern classes. This unified approach will not only accelerate the knowledge acquisition and organization process, but will also formalize and structure the decision making process by reducing the biases of experts. Using this approach, a Knowledge Based ECG Monitoring and Classification System is designed to identify and interpret the ECG signals. The system consists of five primary components, namely, Fact Gathering,

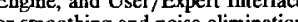
The fact gathering subsystem, 1) preprocess the signals for smoothing and noise elimination, 2) extracts a large feature set from them, and 3) collects the a priori knowledge about the source subject. The facts, a priori knowledge, and the features are organized into a knowledge clustering axt susth. The last subsystem is the Inference Engine which using pattern clustering algorithm. The last subsystem is the Inference Engine which using pattern ecogniton algorithms classifes the analycical knowledge by traversing through the tree and of the proposed system and outlines the implementation details along with some preliminary results. 
39

\section{DISTRIBUTED MEASUREMENT OF VENTRICULAR FUNCTION \\ Waldman, LK}

University of California, San Diego, La Jolla, CA

Over the past ten years, my collaborators and I have developed new methods to measure nonhomogeneous function in the beating heart using implanted markers, biplane $\mathrm{x}$-ray and various strain analyses. Transmural variations of finite strains are measured using closely-spaced columns of beads spanning the wall thickness. Homogeneous strain analysis of tetrahedra has been generalized to a nonhomogeneous finite element technique and used to study mechanical function in the left ventricles of normal canine and porcine hearts as well as pathophysiology associated with acute and chronic ischemia and infarct, pressure and volume-overload hypertrophy and abnormal activation. Epicardial measurements have been extended with surface mapping techniques in which arrays of 25 to 50 markers are sutured to the epicardium and, again, visualized arrays of 25 to 50 markers are sutured to the epicardium and, again, visualized
with $x$-ray. Here, segment shortening from pairs of markers or ultrasonic crystals and two-dimensional homogeneous strains from triplets have been generalized to the study of continuous distributions of two-dimensional strains across substantial regions of the epicardium $\left(16\right.$ to $\left.20 \mathrm{~cm}^{2}\right)$. This technique has been used to study normal function in the left ventricle of pig and dog and the right ventricle of dog and to examine changes in function associated with acute ischemia in pig and its relation to the perfusion border. More recently, this method has been extended further by incorporating a bipolar electrode in each marker. This allows for the simultaneous measurement of both electrical activation and deformation providing a more comprehensive capability to study the effects of delayed activation on the clectro-mechanical function of interacting muscle groups.

\section{0}

INTERACTIONS BETWEEN MYOCARDIAL MECHANICS \& CORONARY PERFUSION Karen D. May-Newman, Jeffrey H. Omens and Andrew D. McCulloch

Institute for Biomedical Engineering, University of California San Diego, La Jolla, CA 92093

Myocardial stress and strain affect the resistance and capacitance of the coronary circulation, and hence the distribution of regional blood pressure and flow. Conversely, alterations in coronary perfusion can affect myocardial wall mechanics in systole and diastole. We suddied the regional mechanics of this interaction in the dog left ventricle (LV). Transmural distributions of 3D strains were measured using columns of radiopaque markers implanted in 8 isolated potassium-arrested hearts, subjected to LV loading duning buffer perfusion at $0-110 \mathrm{mmHg}$.

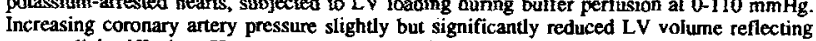
Increasing coronary antery pressure slightly but significantly reduced LV volume reflecting
myocardial stiffening. However, whereas radial and crossfiber strains during filling were myocardial stiffening. However, whereas radial and crossfiber strains during filling were
significanty reduced by perfusion, there was no change in fiber strain. Since most coronary significantly reduced by perfusion, there was no change in fiber strain. Since most coronary
microvessels are parallel to the myofibers, this may reflect changes in microvascular diameter. microvessels are parallel to the myofibers, this may reflect changes in microvascular diameter.
Deformation during perfusion referred to the unperfused state at matched $\mathrm{LV}$ pressure indicated Deformation during perfusion referted to the unpertused state at matched $L V$ pressure indicated
an increase in ventricular wall volume that was transmurally uniform at $50 \mathrm{mmHg}$ perfusion pressure (5\%) but became nonuniform at $110 \mathrm{mmHg}$ with the greatest increase at the endocardium (15\%). Thus regional coronary capacitance in the intact unloaded myocardium was significandly greater $(p<0.05)$ at the subendocardium $\left(0.0016 \mathrm{~mm} \mathrm{Hg}^{-1}\right)$ than the subepicardium $\left(0.00085 \mathrm{~mm} \mathrm{Hg}^{-1}\right)$. This gradient may permit the endocardium to recruit more coronary volume and flow than the epicardium. Similar measurements in the intact hearts of two anesthetized open-chest dogs hefore and after adenosine infusion were consistent with the findings in the isolated heara. To relate these observations to microvascular architecture and
mechanics, we measured capillary diameter and number density at three transmural sites in mechanics, we measured capillary diameter and number density at three transmural sites in
arrested canine hearts perfusion-fixed at coronary artery pressures of 50,80 and $110 \mathrm{mmH}$. arrested canine hearts perfusion-fixed at coronary artery pressures of 50,80 and $110 \mathrm{mmHg}$.
There was no transmural gradient of capillary density or diameter at low pressure but mean There was no transmural gradient of capillary density or diameter at low pressure but mean
values were higher at the subenoocardium than the subepicardium at the highest perfusion pressure. These data forn the hasis of a micromechanical model of perfused myocardium. Supported by NIH grant HL41603 and NSF grant BCS-9157961

41

\section{TORSION EXPERIMENTS ON PAPILLARY MUBCLES}

I Dadmun, N Bichot, JD Humphrey and wC Hunter Dept of Biomedical Engineering, Johns Hopkins University Dept of Mechanical Engineering, University of Maryland Baltimore, Maryland

Excised papillary muscles are convenient for in vitro biomechanical experimentation, but commonly performed uniaxial tests are not sufficient for the complete characterization of the 3D constitutive relation. We show, however, that combined extension and torsion tests provide sufficient multiaxial information to infer a $3 D$ constitutive relation for papillary muscles directly from data. We also describe a new computer-controlled experimental system that we developed for performing these tests on papillary muscles taken from small animals. Finally, we report validations of both the theory and experimental system that were achieved using cylindrical rubber specimens.

\section{2}

MODELS FOR GROWTH AND MORPHOGENESIS IN THE DEVELOPING HEART I-En Lin and Larry A. Taber

Department of Mechanical Engineering, University of Rochester, Rochester, NY 14627

Growth and morphogenesis of the embryonic heart are influenced by biomechanical forces. In the embryonic chick ventricle, for example, increasing ventricular pressure increases the rate of heart growth [1], but the nature of the biomechanical interaction is poorly understood. We developed theoretical models for the embryonic heart to study two developmental mechanisms: the morphogenetic process of cardiac looping and stress-modulated tissue growth. The model for looping (bending of the tubular heart) is a bilayered pseudoelastic beam with one layer representing a longitudinal strip called the dorsal mesocardium (DM), and the other layer is passive myocardium. The DM is given a specified initial tension. As the DM detaches from the body of the embryo during the initial stages of looping, the tension causes the beam to bend, with the myocytes actively changing shape to relieve the tension and the wall stresses. The growth model is a pressurized thick-walled cylinder. Tissue growth is modeled by letting the rate of change of the local zero-stress configuration depend linearly on the Cauchy stresses. The growth response of this model is studied for various constitutive parameters and loading conditions, and experimental data is used to determine growth coefficients. These models provide a starting point for the development of more realistic models for growth and morphogenesis in the embryonic heart. Reference

1. Clark EB, Hu N, Frommelt P, Vandekieft GK, Dummett JL, Tomanek RJ (1989). Effect of increased pressure on ventricular growth in the stage 21 chick embryo, Am J Physiol $257: \mathrm{H} 1-\mathrm{H} 7$

\section{3}

DEVELOPMENT OF LV STRAIN IN HYPERTROPHIC CARDIOMYOPATHY A. A. Young, C. M. Kramer, V. A. Ferrari, L. Axel, and N. Reichok Hospital of the University of Pennsylvania, Philadelphia, PA.

Hypertrophic cardiomyopathy produces markedly heterogeneous patterns of left ventricular wall thickness and myofibrillar disarray. To evaluate regional dynamics during contraction, we have reconstructed the 3D motion of the left ventricle during volunteers, using magnetic resonence tag cardimyopathy ( $\mathrm{HCM}$ ) and twelve nor generated at end-diastole (ED), using spatial modulation of tagging stripes wore autoratically tracked through four or five frames to end-systole (ES) with an active automatically tracked through four or five frames to end-systole (ES) with an
contour model. Once tracked, each stripe point provided a one-dimensional contour model. Once tracked, each stripe point provided a one-dimensional
displacement constraint: the projected distance from the deformed position to the displacement constraint: the projected distance from the deformed position to the
original tagging plane. A 3D finite element model of the LV was used to combine this information from tagging planes of three or four different orientations and thus reconstruct 3D displacement and strain. Strains were calculated at two times relative to ED: one at earty systole (25-33\% from ED to ES) and one at ES. While radial motion (towards the long axis) was nomal in earty systole and slightly reduced at ES, the longitudinal motion of the base towards the apex was markedly reduced at both times. The normal anticlockwise rotation about the long axis during early systole was preserved at the apex $\left(5.6 \pm 2.8^{\circ} \mathrm{HCM}\right.$ vs. $5.2 \pm 1.4^{\circ}$ control) but abolished at the base in HCM patients $\left(-0.1 \pm 1.1^{\circ}\right.$ vs. $\left.2.4 \pm 1.2^{\prime}\right)$. Torsion (defined as rotation about the long axis relative to that of the base) was increased at both early and end-systole, especially in the apical posterior wall $\left(7.3 \pm 3.1^{\circ}\right.$ vs. $3.1 \pm 1.3^{\circ}$ at earty systole). The least principal strain (associated with grealest contraction) was slightly increased at early systole, but depressed at ES in the mid-ventricular and basal septum $(-0.18 \pm 0.05$ vs. $-0.22 \pm 0.02$ in the basal septum at ES). In conclusion, principal contraction was preserved at earty in the basal septum at ES). In conclusion, principal contraction was preserved at earty
systole but reduced in the septum at ES. Longitudinal shortening was reduced and lorsion enhanced throughout systole.

44

ALTERING THE FILLING BEHAVIOR OF AN IN VITRO EXCISED PORCINE heart PREParation D Melvin, H Schima, U Lossert, H Stöhr, H Siegl, L Huber, E Wolner. Center for Biomedical Research, Ludwig Bolzmann Institute and 2nd Dept. of Surgery, Univ Vienna, Vienna, Austria, and Dept. of Surgery, Univ Cincinnati, Cincinnati, $O H$. Mechanical 're-powering' of a failing heart with devices or skeletal muscle could potentially circumvent blood-pump lining problems. Such techniques are difficult to explore experimentally. There is no close physicgl simulation of a dilated, end-stage, human heart to use for testing and refining procedures prior to in vivo trials. The complex requirements (effective support while preserving valve closure and coronary llow, avoiding wall coaptation
and still allowing both rapid low impedance re-filling and independent left and right pressures) demand a testing preparation with detailed features not readily acbievable with prosthetic models. Transplant recipient hearts (the exact target population) are not usually excised with all valves and vessels intact. Pig hearts are anatomically similar to nomal but not to dilated human hearts, and their compliance falls rapidly after death. Most fixatives fruther decrease compliance. Physiologically adequate in vivo animal failure preparations have not reproduced patbologic geometry. Mechanical and/or chemical alteration of ventricular anatomy and material characteristics was thus attempted in 76 hearts from $90-120 \mathrm{~kg}$ swine with particular attention to filling mechanics. Fresh slaughter-house hearts were dilated after cardioplegia at $0-4,18-20$ or $35-38^{\circ} \mathrm{C}$ using direct ventricular fluid inflation or one of several balloon designs. After unacceptable stiffness with formaldehyde and inadequate preservation with glutaraldehyde perfusion, a series of 16 balioon-dilated hearts with stent-protected valves were perfused with ethyl alcohol. Dimensions were assessed by ultrasound and mechanical behavior by continuous pressure/volume relationship during 24 to 72 hours of cyclic filling. Geometry (apptoximate ellipsoid, full volume $120-160 \mathrm{ml}$ ), valve function (no visible leak in 4 of 5 tested at $100 \mathrm{mmHg}$ ) and low-volume compliance $(2100 \mathrm{ml}$ filling from a somewhat flattened state with $\leq 5 \mathrm{mmHg}$ pressure increment) appear sufficiently similar to failing human hearts to be useful for in vitro appraisal of filling and valvular function during mechanical wall activation methods. 
45

DISSOCIATION OF LEFT VENTRICULAR AND INTRAMYOCARDIAL PRESSURES IN ISOLATED RABBIT HEARTS

Hiroshi Yamada and Frank C.P. Yin

Johns Hopkins Medical Institutions, Baltimore, MD

Because LV cavity pressure (LVP) is a boundary condition for the myocardial wall, intramyocardial pressure (IMP) is thought to be related to LVP. The heterogeneous material, complex structure, and nonlinear mechanical behavior of the tissue components at the microscopic level, however, suggest that this concept may be an oversimplification. To further test this, we measured IMP in isolated rabbit bearts in diastole and during barium contracture with a servo-null system using glass pipettes $<10$ microns in diameter. In diastole $(\mathrm{n}=6)$ at constant coronary crystalloid perfusion pressure of $47 \mathrm{mmHg}$, IMP in the unloaded and highly loaded states (LVP of $1.2+0.5$ and $31.8 \pm 2.2 \mathrm{mmHg}$; mean \pm SEM) was $6.7 \pm 0.9$ and $10.5 \pm 1.4 \mathrm{mmHg}$. In contracture $(n=5)$ at the same perfusion pressure, IMP in the unloaded and loaded states (LVP of $2.4 \pm 0.8$, and $31.1 \pm 4.2 \mathrm{mmHg}$ ) was $21.7 \pm 4.1$ and $24.7 \pm 4.9 \mathrm{mmHg}$. To further show that changes in IMP do not require changes in LVP, in two hearts we held LVP at nearly zero pressure and changed coronary perfusion pressure from 50 to $70 \mathrm{mmHg}$. IMP was 6.9 and $8.1 \mathrm{mmHg}$ at these perfusio prescures, Since IMP was higher than LVP in both unloaded distolic and systolic stares of LVP, intramyocardial pressure can not be directly related to left ventricular pressure.

46

STATUS REPORT ON THE DEVELOPMENT OF A RIGHT-

VENTRICULAR-VOLUME-MEASUREMENT INSTRUMENT

Peter J. Yim, Timothy A. Johnson, Ph.D., Carol L. Lucas, Ph.D. The Department of Biomedical Engineering, The University of North Carolina at Chapel Hill

An instrument based on sonomicrometry is needed for measuring the heart's right ventricular volume in animal experiments studying the elasticity and contractility of the right ventricle (RV). The feasibility of determining the RV volume based on several ventricular dimensions was assessed using five casts of lamb RV's. The product of the anteriorposterior dimension and the septal-free-wall dimension of the lamb heart was found to have a good correlation with the RV volume $(r=0.954)$. Using seven excised porcine hearts, a method for controlling the RV volume, for use in validation studies, was developed that relies on a frequently calibrated volumepressure relation. Dimensions of the porcine hearts were measured with sonomicrometry (based on transit-time between two crystals) but were considered unrealistic due to rigor mortis of the hearts. 5-MHz-planar-crystal transducers were unreliable in in vitro testing due to insufficient beamwidth. Tests of transducer beamwidth conducted in water indicate that 2-3-MHzplanar-crystal transducers will have better performance. We conclude that the critical choices in the design of this instrument are which dimensions to measure and which transducers to use to make those measurements.
47

A THREE DIMENSIONAL SIMULATION MODEL OF SYSTOLIC FLOW FOR EVALUATION OF LEFT VENTRICULAR FUNCTION

Erick A. Gonzalez and Richard T. Schoephoerster

Mechanical Engineering Department, Florida International University, Miami, FL 33199

Left ventricular (LV) dysfunction due to coronary artery occlusion is traditionally evaluated through global geometric measurements such as stroke volume and ejection fraction. We have previously computed the LV chamber 2-D systolic velocity distribution using as input to the model the time-varying LV wall motion obtained from right anterior oblique (RAO) ventriculograms, and introduced a quantitative parameter, Central Ejection Region (CER) coefficient, which will characterize global left ventricular function based on the simulated flow dynamics computed from and sensitive to regional, time-dependent LV wall motion. The CER coefficient provides a qualitative and quantitative measure of global function that regional wall motion analysis cannot provide, and is a parameter which is sensitive to regional and temporal abnormalities and the resulting compensatory actions which cannot be detected by global parameters. In order to provide more realistic and functional LV systolic flow dynamics, as well as the ability to localize and evaluate the condition of the three dimensional for the developed. The thee dinensies of six two dimensional echocardiographic apical long axis views obtained by rotating the ultrasound transducer through 30 degree intervals. The continuity and three dimensiona Navier-Stokes equations are discretized using the finite analytic method and the solution obtained using boundary conditions computed from the regional wall motion. A parametric study was performed comparing the resulting CER from a "perfectly" contracting elliptical LV with several simulations of "abnormal" wall motion. Results indicate that the CER is parameter which will be sensitive to the three dimensional motion of the LV wall and effective in evaluating LV function as a means of assessing the efficacy of anti-thrombolytic therapy. 
49

CORONARY MICROVASCULAR RESPONSES TO PRESSURE AND FLOW. Lih Kuo, Michael J. Davis, and William M. Chilian.

Department of Medical Physiology and Microcirculation Research Institute, Texas A\&M University Health Science Center, College Station, TX 77843.

The goals of this study were to examine pressure-and flow -induced microvascular responses and to delineate the mechanisms of these responses in terms of the involvement of endothelium and its derived vasoactive substances. Porcine subepicardial arterioles and venules, $60-100 \mu \mathrm{m}$ internal diameter, were isolated and cannulated with two glass micropipettes and then pressurized via independent reservoir systems while measuring vessel diameters using video microscopy. T study the vascular responses to intraluminal pressure (IP) changes, i.e. myogenic responses, IP was altered by moving both reservoirs in the same direction. To activate flow-dependent responses, lumenal flow was increased by simultaneously moving the reservoirs in equal but opposite directions, thus generating a pressure gradient across the vessel without changing the mean IP. In the absence of flow, all vessels developed spontaneous tone. Varying IP within the ange 20 to absence of flow IP increases and decreases, respectively. In contrast, coronary venules responded passively to changes in pressure $\left(2-40 \mathrm{cmH}_{2} \mathrm{O}\right)$. Pressure-diameter relationships of arterioles and venules were not altered after disruption of the endothelium. Both coronary arterioles and venules dilated in response to the increases in lumenal flow. Flow-induced rasodilation wa completely abolished after endothelial removal. Bioassay studies, using intact and denuded arterioles connected in series, indicated that a transferable substance was released from the intact endothelium in response to flow. Flow-induced dilation was not affected by indomethacin, a prostaglandin synthesis inhibitor, but was abolished by $\mathrm{N}^{\mathrm{G}}$-monomethyl-L-arginine, a selective and competitive inhibitor of nitric oxide synthesis. Our results indicate that coronary anterioles possess stronger myogenic responses than those in venules, and these responses ane endotheliumindependent nitrovasodilator, rather than a prostanoid, from endothelial cells.

50

EFFECTS OF MAGNETIC RESONANCE CONTRAST AGENTS IN MYOCARDIAL TISSUE Robert M. Judd, Elliot R. McVeigh, Anne Bazille, Dikoma C. Shungu, Elias A. Zerhouni Departments of Radiology and Biomedical Engineering, The Johns Hopkins University

Magnetic resonance (MR) can in principle be used to measure regional myocardial flow, vascular volume, extracellular volume, and capillary permeability. However, there is a lack of uderstanding concerning the relationship between contrast concentration and the resulting myocardial MR signal. We have developed a model in which this relationship can be studied at a fundamental level. An isolated canine interventricular septum is pertused at room temperature with cardioplegic solution producing a viable but non-beating specimen. A surface $R F$ coil is placed on the septum to directly detect the myocardial MR signal every $300 \mathrm{msec}$ during a train of $90^{\circ}$ RF pulses, allowing us to calculate $T 2^{\circ}$ and measure $T 1$ enhancement simultaneously for each RF pulse. Contrast agents are injected as boluses directly into the cannulated septal artery while coronary pressure and flow are continuously monitored. The $T 1$ effects of albumin-GdDTPA (A) and Gd-DTPA (B) are shown in the left-hand Figure. T2* for these same injections are shown in the right-hand Figure. The T1 curves are similar to classical indicator-dilution curves in that large molecules, such as albumin, remain intravascular while smaller molecules, such as DTPA, extravasate on the first pass through the coronary circulation. The $T 2^{\circ}$ curves, however, contain additional information about the
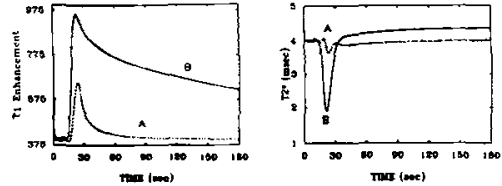
vasculature.

51

EXTRAVASCULAR COMPRESSIVE PRESSURE PREDICTED FROM EPICARDIAL CORONARY FLOW DYNAMICS

S. Sideman, D. Manor, R. Shofti and R. Beyar

Technion-Israel Institute of Technology, Haifa, Israel.

The forces exerted by the myocardium on the intramyocardial circulation, defined here as the extravascular compressive pressure (ECP), can be predicted from epicardial coronary flow measurements under constant coronary perfusion pressure using an appropriate perfusion tine model. We have characterized the shape of the ECP under these conditions and compare it with the shape of direct high fidelity measurements of left ventricular pressure (LVP) and midwall intramyocardial pressure (IMP) waveforms. Coronary arterial flow was measured in 10 mongrel dogs at constant perfusion pressure. A non-linear analog capacitive-resistive coronary perfusion model was used to determine the ECP function. The calculated ECP phasic waveforms are mirror images of the corresponding measured flow signals. Typically, the ECP wave shows a continuous systolic increase peaking close to end-systole. The shape of the phasic ECP waveform resembled the shape of the experimentally measured IMP waves, but was different from the LVP waveforms. A decrease in LVP by preload manipulation affected both the ECP shape and the amplitude, while a change in the perfusion pressure modified only the ECP amplitude but not its shape. It is suggested here that the ECP function, calculated based on measurements of epicardial flow under conditions of constant perfusion pressure, represents an index reflecting the effective compression of the contracting myocardium on the intramyocarcial circulation. The ECP differs markedly in shape from the LVP and resembles the IMP measurements.
52

INTRACORONARY PRESSURE DURING RADIOGRAPHIC CONTRAST INJECTION Maria Siebes', Kimberly A. Herman ${ }^{1}$, James D. Rossen ${ }^{2}$ Departments of Biomedical Engineering ${ }^{1}$ and Internal Medicine ${ }^{2}$, The University of lowa, Iows City, IA 52242

Coronary arteriography is widely regarded as the 'gold standard' for the quantitative assessment of coronary atenosis severity. Known pitfalls in the analysis include effects of vasoactive agents, poor contrast mixing, and changes in intraluminal pressure. Little attention has been paid to possible fluid mechanical effects caused by the necessary injection of contrast material into the coronary artery. Transfemoral coronary angiography was performed in healthy pigs. A $1.5 \mathrm{~F}(0.5 \mathrm{~mm}$ o.d.) catheter-mounted micro-tip pressure transducer was placed inside an $8 \mathrm{~F}$ ( $2.01 \mathrm{~mm}$ i.d.) coronary injection catheter and advanced into the left circumflex coronary ortery to measure intracoronary pressure during administration of $8 \mathrm{ml}$ of radiographic contrast material. Pressures were mensured at the tip of the injection catheter and 1,2 and $2.5 \mathrm{~cm}$ distal to the tip. Power injections at rates of $2,3,4$, and $6 \mathrm{ml} / \mathrm{sec}$ as well as hand injections were carried out repeatedly. lnitial results show a substantial elevation in coronary pressure during the period of contrast administration. The pressure incressed progressively with incteasing rate of injection, up to a rise of $30 \mathrm{mmHg}_{\mathrm{g}}$ for hand injections. A similar relationship was found with increasing distance from the tip of the injection catheter. However, the data at larger distances were obtained at higher pre-injection pressures, at which the arterial wall is less compliant and therefore, intracoronary pressure is more responsive to rapid volume losding. In conclusion, these results anggeat that the intracoronary injection of radiographic contrast material gives rise to a temporary increase in coronary pressure, which appears to depend on the rate of injection and the elastic properties of the artery. Since this artificial elevation of coronary pressure oceurs daring the primary phase of image acquisition, it may very well lead to subsequent erroneous estimates of coronary dimensions by quantitative angiogram analysis.

\section{3}

SYSTOLIC-DIASTOLIC CHANGES IN INTRAMYOCARDIAL VASCULAR VOLUME Kevin K. Yam, Robert M. Judd, and Frank C. P. Yin

Johns Hopkins Medical Institutions, Baltimore, MD

Coronary inflow and outflow are out of phase during systole, indicating a net decrease in coronary vascular volume during contraction. The magnitude of this diastolic/systolic variation in vascular volume provides an important insight into the coupling between the vasculature and muscle. However, direct measurements of intravascular volume have not been made. We used our previously described digital subtraction angiography (DSA) method to measure this variation in 3 isolated, perfuser canine interventricular septa. Every $200 \mathrm{msec}$ intramyocardial vascular volume was calculated from the change in pixel intensity within myocardial tissue due to an intravascular contrast agent (perfluorooctylbromide). The figure shows phasic septal flow and corresponding vascular volume during cardiac contraction in one specimen. On average, vascular volume fell from $14.2 \pm 4.3$ to $7.4 \pm 5.1 \mathrm{~m} / 100 \mathrm{~g}(51 \pm 24 \%)$.
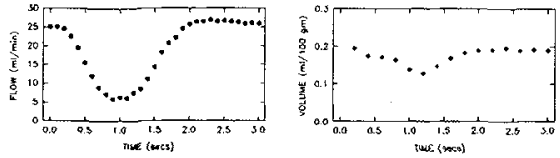

The data show that cardiac contraction can expel a significant fraction of the intramyocardial volume. While the magnitude of these volume changes may differ from those in-vivo, these changes in intramyocardial volume suggest that cardiac contraction directly affects the microvasculature.

54

MYOCARDIAL BLOOD FLOW AND REGIONAL CARDIAC FUNCTION: EFFECTS OF EXERCISE TRAINING. J. L. Hall, P. A. Harris, C. H. Lorenz, M. H. Laughlin, and K. A. Overhalser. Vanderbilt Univ., Nashville, TN 37235, and Univ. of Missouri, Columbia, MO, 65211

Magnetic resonance imaging (MRI) was used to study regional cardiac function and distribution of coronary blood now in two Yucatan miniature swine before and after 16-week period in which one pig remained sedentary while the other underwent a treadmill exercise training program. Tagged (grid pattern) cine imaging was used to determine left ventricular (LV) myocardial surain. The exercise-trained pig showed a $48 \%$ increase in mean $\mathrm{LV}$ wall displacement after the training period, while the sedentary animal showed an $11 \%$ increase, suggesting an effect of exercise training on ventricular wall function. Regional myocardial perfusion was studied with dynamic contrast-enhanced MRI. A T1-weighted turboFLASH imaging sequence was used to track signal intensity changes induced by intravenous injection of $0.1 \mathrm{mmol} / \mathrm{kg} \mathrm{gd-}$ DTPA. Image acquisition time was approximately $0.5 \mathrm{sec}$ acquisition continued for $30 \mathrm{sec}$. Signal intensity-time curves (normalized to baseline) were calculated for each of 36 myocardial segments and integrated. The area under each curve was considered to be related to blood volume in a sector. It was found that exercise training led to a higher normalized area ( $53.9 \pm 7.8$ post-training vs $45.0 \pm 2.6$ pre-training) and to a more heterogeneous myocardial flow, elevated in inferior wall and septal regions. The zome of higher blood volume corresponded to the sectors of greatest increase in ventricular wall function, suggesting simultaneous, localized, training-induced alteration in coronary flow and mechanical work. Supported by HL-36531 and HL07411 NRSA. 
55

SELF-EXCITED OSCILLATIONS IN COLLAPSIBLE TUBES: EFFECTS OF TUBE TENSION AND DOWNSTREAM RESISTANCE HT Low, YT Chew, SH Winoto, " ${ }^{\circ} \mathrm{CN}$ Lee and JW Wang Department of Mechanical \& Production Engineering

National University of Singapore, * Gleneagles Medical Centre, Singapore

Flow instability in collapsible tubes may be of interest in relation to that in the natural veins, and the Korotkoff sound generation in collapsed arteries. The mechanism initiating self-excited oscillation has been associated with the downstream characteristics, critical flow, separated flow and panel flutter, but is not yet fully understood. This paper studies the effect of tube tension and downstream resistance on the pressure-flow behaviour of collapsible-tubes and its' self-excited oscillations. One of the main features of the present experiments is that the various transmural pressures are varied systematically whilst keeping other parameters fixed, so that the effects of each parameters are clearly interpreted. The tube of diameter $10 \mathrm{~mm}$ and length 120 $\mathrm{mm}$ was attached to rigid ducts at both ends and surrounded by a pressurized chamber. The initial tube tension corresponded to $0-25 \%$ strain, and the external pressure was kept fixed at $40 \mathrm{~cm}$ water. The upstream transmural pressure was varied at $\pm 3 \mathrm{~cm}$ water and the downstream transmural pressure varied down to $-20 \mathrm{~cm}$ water. Results are presented of the pressure-drop versus flow behaviour for various values of upstream transmural pressure. For positive upstream transmural pressure there is a negative pressure-flow gradient, indicating flow instability. The effect of tube tension is similar to an increase of transmural pressure. A low downstream resistance has the effect of initiating tube oscillation; measurements were made of the frequency and amplitude of the flow and pressure oscillations. The various tube oscillation modes are presented through video-recording.

56

\section{TWO-DIMENSIONAL VELOCITY FIELD MEASUREMENTS USING COLOR DOPPLER ULTRASOUND}

\section{R.S.C. Cobbold, T.A. Maniatis, M. Ojha, and K.W. Johnston} Institute of Biomedical Engineering,

University of Toronto, Toronto, M5S 1 A4, Canada

Color flow Doppler ultrasound images from complex vessel geometries can be difficult to interpret, thereby limiting the effectiveness of the technique to correctly assess geometric abnormalities. and to quantitatively relate the images to the underlying flow field. This paper describes progress in calculating and displaying two-dimensional velocity vectors from an end-to-side anastomosis model under steady flow conditions at various Reynolds numbers. A linear array Doppler system enables color flow images to be recorded from three incident beam directions in the plane of symmetry of the model. The velocity vector field within the plane of view is then calculated from the digitized images. It is shown that the calculated velocity magnitude image provides a relatively simple interpretation of the flow field. In addition, velocity vectors superimposed on one of the Doppler images provide a means of understanding the complex color-coded flow pattern. The secondary velocity field was calculated and compared to the theoretical values for flow in curved vessels. Finally, results are discussed in relation to flow visualization observations of the three-dimensional velocity field in similar anastomosis models at comparable Reynolds numbers.

\section{7}

INTEGRATED EXPERIMENTAL AND MUMERIGAL SIMULTATION APPROACH TO LEFT ATRIAL BIODYNAMICS+

Urmila Ghia, Ph.D., Li-H. Chen, and Brian D. Hoit, M.D. University of Cincinnati, Cincinnati, $O H$

With the objective to acquire an improved understanding of left atrial (LA) fluid dynamics, LA flow is represented by the complete unsteady Navier-stokes equations and simulated numerically using a novel temporally deforming adaptive grid. The system geometry was idealized to consist of an ellipsoidal LA chamber, a composite pulmonary ve in (PV) and a rigid mitral valve. Preliminary results have been obtained for the configuration with the mitral valve in the fully open position and clearly show a circulatory instantaneous flow pattern in the atrial chamber. The approach is capable of modelling unsteady flows, including the effects of temporal variations in geometrical dimensions as well as inflow and outflow boundary conditions. Accordingly, in a parallel effort, experimental data have been acquired for canine LA flow by instrumenting animals with a high fidelity LA micromanometer, pairs of sonomicrometers on the long and short axes of the LA and on the PV, and a PV flow probe. Computational results are presently being obtained for these measured temporally varying conditions. This research has the potential for general cardiac flow analys is and therefore may a id in development/improvement of cardiac prosthetic devices.

+ Supported in part by a UC Biomedical Engineering Seed Grant: resources in part provided by the Ohio Supercomputer Center.
58

Interpretation of Flow Velocity Measurements from a $12 \mathrm{MHz}$ Doppler Guidewire Downstream of a Coronary Stenosis Model Helene Leclerc ${ }^{*}$, Steven A. Jones ${ }^{+}$, Neal A. Scott ${ }^{\dagger}$ - Georgia Institute of Technology, Atlanta, GA, + Johns Hopkins University, Baltimore, MD,

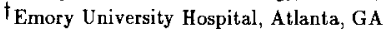

The shape of a Dopples ultrasound spectrum depends on the transducer beam pattern, and this can be altered by the acoustic properties of blood and tissue. To account for this, a model of a coronary artery stenosis has been constructed such that the blood analogue fluid and the model material have identical acoustic impedance. Thus, alterations of the beam pattern are minimized. The resulting Doppler power spectra are interpreted in terms of known fluid mechanics downstream of a stenosis. Three stenoses were studied with cross-sectional area reductions of $50 \%, 75 \%$ and $90 \%$. Reynolds numbers were 240 and 400 .

The separation region downstream of the stenoses exhibited three peaks in the Doppler spectrum: one at high frequency correspondind to the velocity of the jet; one at low negative frequency, corresponding to the reverse flow; and one at low positive frequency, corresponding to the forward flow part of the recirculation region. The relative sizes of these peaks changed with axial distance downstream of the constriction. For example, the power of the high frequency peak diminished in amplitude with distance from the stenosis. This reduction in power was gradual for the $50 \%$ stenosis, but its rapid occurrence for the $75 \%$ and $90 \%$ stenoses probably reflects transition to turbulence.

The Doppler spectra could be readily interpreted in terms of known fluid mechanical phenomena caused by a stenosis only when the acoustic impedance of the model and the blood-analogue fluid were matched. In vivo, where acoustic impedance match is not guaranteed, it may be necessary to compensate for acoustic phenomena to correlate Doppler measurements with fluid mechanics.

59

In-Vitro Measurements of Wall Shear Stress in a Nearly Symmetric Left Coronary Artery Bifurcation Model

Leigh A. Miller ${ }^{*}$, Steven A. Jones ${ }^{+}$, Don P. Giddens ${ }^{+}$, Chris K. Zarins ${ }^{\dagger}$, Seymour Glagov ${ }^{\dagger}$ -Georgia Institute of Technology, Atlanta, GA, ${ }^{+}$Johns Hopkins University, Baltimore, MD, † University of Chicago, Chicago, IL

The geometry of the left coronary artery bifurcation varies greatly from individual to individual. To establish general relationships between artery wall shear stress and atherosclerosis or intimal hyperplasia, one must know how this variability affects wall shear stress. Thus shear stress estimates were obtained by laser Doppler anemometry from a nearly symmetrical left coronary bifurcation model and compared to earlier measurements by Tang in a similar, but non-symmetric model. The models have the same branch diameters, and exhibit physiological branch curvature, and bifurcation angles. They include the left main, left anterior descending and left circumflex arteries.

For both models, wall shear stress was generally higher along the epicardial surface than along the myocardial surface and higher along the inner walls of the branches than along the outer walls. A larger turning angle into the circumflex artery of the non-symmetric model caused the shear stress along the outer wall of this branch to be approximately half as large in the non-symmetric model as in the symmetric model. Recirculation in the symmetric model only, along the myocardial wall midway through the left main is attributed to a sharper left main entrance from the ostium in the symmetric model.

If artery wall shear stress is inversely related to intimal thickening, then both models imply increased thickening along the myocardial wall and along the outer walls of the branches. However, the model with the higher turning angle into the circumflex artery should be more prone to intimal thickening along the outer wall of this branch. Since the curvature in the left main did not differ greatly between the $t$ wo models, the presence of recirculation in one and not in the other indicates a strong influence of ostium geometry.

60

EXPERIMENTAL, STUDY OF STEADY FLOW IN RIGID MODELS OF ABDOMINAL AORTIC ANEURYSMS

C.L. Asbury', J. Ruberti', R.A. Peattie', and E.I. Bluth ${ }^{2}$

${ }^{1}$ Dept. of Biomedical Engineering, Tulane University, and ${ }^{2}$ Dept. of Radiology, Alton Ochsner Medical Foundation, New Orleans, La 70118

It has been estimated that abdominal aortic aneurysms occur in as much as $2-3 \%$ of the population, and that their rupture produces a mortality rate between $78-94 \%$. As an investigation into the mechanical factors which lead to aneurysm nupture, flow field measurements for steady flow are presented for a range of aneurysm sizes and Reynolds numbers. Seven rigid models with elliptical aneurysm geometry were constructed with uniform lengths of $4 d$ and of diameters that ranged from 1.5 to $3.0 d$, where $d$ is the inner diameter of the undilated entrance tube. Color Doppler flow imaging was used to qualitatively characterize the flow fields and to provide information regarding the onset of, and complete transition to, turbulent flow. In addition, Laser Doppler Velocimetry was used to quantify the flow field velocities and to estimate wall shear stress values for each of the models. It was found that over a range of Reynolds numbers corresponding to in yive flow rates, the flow can be either laminar or turbulent. Both visualization images and velocity profiles are presented, and conditions for transition to turbulence are discussed. This investigation represents an initial step in a study of flow fields within abdominal aortic aneurysms, the results of which will have significant implications for clinical decisions concerning the treatment of aortic aneurysms.

Acknowledgments - The authors gratefully acknowledge the support of the Louisiana Educational Quality Support Fund and the Whitaker Foundation. 
61

BLOOD VOLUME SHIFT FROM MICROCIRCULATION AS THE MAJOR COMPENSATION TO MACROVASCULAR BLOOD VOLUME LOSS BY HEMORRHAGE J. S. Lee and L. P. Lee

Dept. Biomed. Eng., Univ. Va. Charlottesville, VA 22908

We measured the changes in blood and plasma density to assess macrovascular volume compensation resulting from hypovolemia via fluid restitution from transcapillary exchange and blood volume shift from micro- to macrocirculation. Rabbits conscious (CONS) and then anesthetized (ANES) were subject to a cyclic hemorrhage protocol with $\Delta V$ as the volume
variation and a cyclic period of 2 minute. With the consideration that transcapillary exchange variation and a cyclic period of 2 minute. With the consideration that transcapillary exchange plasma density the variation in the volume of fluid restitution. This volume was found to be $7 \%$ of $\Delta \mathrm{V}(\mathbf{r}=0.98)$. Calculated from the blood and plasma density variation at $10 \%$ hemorthage, the hematocrit variation was $1.19 \pm 0.04 \%$. In view that the addition of restituted fluid to the circulating blood could only produce a hematocrit variation of $0.25 \pm 0.03 \%$, difference from the observed hematocrit variation was used to determine the microvascular volume shift based on the consideration that the microcirculation had a microvascular hematocrit lower than the hematocrit of arterial blood. This volume shift was $58 \%$ of $\Delta V$ ( $r=$ 0.99 ). These two volume relations were not altered by anesthesia. Despite different mechanisms controlling the arterial pressure for CONS rabbits with and without the infusion of hexamethonium (HEX) and ANES rabbits, we estimated from the measurement of total peripheral resistance and a two resistor circulation model that microcirculatory pressure could be similar. Thus the volume variations of fluid restitution and microvascular shift were linearly related to the variation in microcirculatory pressure with the relations unaltered by $\mathrm{HEX}$ or ANES. From these results, we conclude that the microvascular volume shift is the major short term mechanism compensating the hemorrhaged volume. The mechanism is not by direct neural control or local regulation but by a passive elastic microcirculation responding to microvascular pressure reduction. Supported in part by NHLBI grant HL 40893 .

62

HEPATIC CAPACITANCE RESPONSE TO HEMORRHAGE IN THE CAT.

C.V. Greenway

Dept of Pharmacology, Univ. of Manitoba, Winnipeg Canada.

Cats anesthetized with pentobarbital were hemorrhaged $\left(1 \mathrm{ml}^{-m^{-1}} \mathrm{~kg}\right.$ body $\left.\mathrm{wt}^{-1}\right)$ until arterial pressure declined to $55 \mathrm{~mm} \mathrm{Hg}$ while hepatic volume was recorded by plethysmography. Time controls showed that 3 hemorrhages at $1 \mathrm{~h}$ intervals gave comparable responses in which the liver contributed $20 \%$ to a mean hemorrhage of 20 comparable responses in which the liver contributed $20 \%$ to a mean hemorrhage of 20
$\mathrm{~m} / \mathrm{kg}$. Twelve series of experiments were done in which hepatic or splanchnic nerves were cut, venous or arterial pressures were manipulated, or afferent (carotid, vagal) reflex mechanisms controlled. No evidence for a role for the hepatic nerves was found; and liver volume responses did not correlate with changes in portal or hepatic venous pressures. An explanation for the results is not obvious. However when the experimental procedure changed the volume of hemorrhage required to reduce arterial pressure to $55 \mathrm{~mm} \mathrm{Hg}$, the liver volume response changed proportionately and the hepatic contribution remained $20 \%$ $\left(P<0.0001 ; r^{2} 0.6 ; n=163\right)$. The data and some possible interpretations will be presented (Supported by MRC of Canada and Manitoba Heart and Stroke Foundation).

63

BLOOD VOLUME DISTRIBUTION OF INTESTINAL MICROCIRCULATION AND ITS CONTROL BY THE BARORECEPTOR REFLEX SYSTEM A. A. Shoukas and E. B. Haase, Dept of Biomedical Engineering, The Johns Hopkins University School of Medicine, Baltimore, Maryland.

Recent publications have shown that the sympathetic nevous system and the carotid sinus baroreceptor reflex system have the ability to regulate the pressure-diameter relationships of the small veins and venules of the intestine of the rat. In order to relate the changes in the pressure-diameter relationships of individual vessels to changes in blood volume of an organ it is necessary to have a complete architectural description of the intestinal vascular bed as well as knowledge of the responsiveness of the arteries and arterioles. We obtained the transient arteriolar pressure-diameter relationships, the steady-state arteriolar diameter relationships during activation of the baroreflex system and morphological data on the rat intestinal vascular bed. These data were used to allow for a quantitative prediction of the changes in intestinal microcirculatory total blood volume. The results revealed that venules hold $70 \%$ of the total microcirculatory volume and are responsible for approximately $80 \%$ of the total blood volume shift when the baroreceptor reflex is activated. Arteriolar constriction results in a $14 \%$ decrease in microcirculatory blood volume. Thus, volume changes within the venules play an important role in intestinal capacitance.
64

MODEUNG ACUTE HEMORRHAGE IN THE HUMAN CARDIOVASCULAR SYSTEM Stefanie Neumann

University of Pennsylvania, Department of Bioengineering

For exsanguinating emergency patients, the critical factor determining the time available for preoperative diagnosis and intervention is the patients' rate of bleeding. Over time, massive blood loss may induce shock and ultimately death. The author's aim is to develop a computationally efficient, firstprinciples model of acute cardiovascular (CV) response to exsanguinating trauma and fluid replacement to aid physicians in making crucial decisions. The model is unique in two respects: 1) No published models consider acute hemorrhage; 2) Because precise values for significant CV parameters are unavailable in an Emergency Center, the model will be a mixed quantitative/ qualitative $(\mathrm{Q} / \mathrm{Q})$ model, capable of using qualitative estimates of those parameters. Qualitative reasoning allows system simulation when not all system pararneters are known precisely. The accuracy of the mixed $Q / Q$ model's results improves monotonically with the accuracy of its input.

The purely quantitative model depicts the pulsatile nature of blood flow throughout a closed system, and includes major systemic compartments, such as arterial and venous pulmonary and systemic partitions, and left and right hearts. System relationships which cannot be precisely defined are converted into a set of constraints, and parameter domains and landmark values are specified, producing the $Q / Q$ model. This model operates as a continuous, timedependent cycle that forms expectations by projecting statistics into the future and uses differences between expected and actual (i.e., monitored) observations to update the model. The updates ensure that the expectations become increasingly accurate, providing a solid basis for clinical decisions and activity.

65

INFLUENCE OF NITROGLYCERIN ON SPLANCHNIC VOLUME AND CARDIAC OUTPUT M.A. Morse and D.L. Rutlen Section of Cardiology, Medical College of Georgia, Augusta, GA

The present study examined the importance of changes in spianchnic capacity, as assessed by scintigraphy, in the regulation of cardiac output (CO) during nitroglycerin in 16 anesthetized pigs after denervation. With nitroglycerin $(0.5 \mathrm{mg} \mathrm{min}-1$ i.v.) for $5 \mathrm{~min}$, BP decreased from $115 \pm 7$ to $95 \pm 7 \mathrm{mmHg}(P<.0001)$, portal vein pressure decreased from $9.0 \pm 0.5$ to $8.5 \pm 0.5 \mathrm{mmHg}(P<.0001)$, portal flow increased from $637 \pm 49$ to $668 \pm 60 \mathrm{ml} \mathrm{min}^{-1}(P=.09)$, and transhepatic resistance decreased from $7.5 \pm 1.5$ to $6.5 \pm 1.0 \mathrm{mmH}$ min $7^{-1}$ $(P<.01)$, but $C O$ was unchanged $\left(1929 \pm 126\right.$ to $\left.1890 \pm 138 \mathrm{ml} \mathrm{min}^{-1}\right)$. Total splanchnic intravascular volume $\left(V_{j}\right)$ increased $1.6 \pm 1.0 \%$ $(P<.05,14 \pm 10 \mathrm{ml})$. This increase was due to an increment in mesenteric $V_{i}(12.9 \pm 1.9 \%, P<.0001)$, since splenic $V_{j}$ decreased $(9.6 \pm 2.8 \%, P<.0001)$ and hepatic $v_{i}$ did not change. After splenectomy, nitroglycerin at $0.5 \mathrm{mg} \mathrm{min}^{-1}$ and $2 \mathrm{mg} \mathrm{min}^{-1}$ was associated with increases in total splanchnic $V_{i}$ of $3.7 \pm 1.2 \%$ $(P<.0001,30 \pm 10 \mathrm{ml})$ and $7.6 \pm 1.7 \%(P<.001,59 \pm 10 \mathrm{ml})$ due entirely to increases in mesenteric volume of $9.9 \pm 2.7 \%$ $(P<.0001)$ and $16.5 \pm 1.9 \%(P<.0001)$, respectively, but $c 0$ was unchanged. Evisceration did not alter the co response. Thus, nitrogiycerin is associated with an increment in total splanchnic $V_{j}$ due to active mesenteric venodilation and an increase in portal flow. The splanchnic $V_{j}$ changes do not affect $c 0$.

66

VASCULAR CAPACITANCE IN EXPERIMENTAU HYPERTENSION M.J. Brunner, G.G. Bishop, K. Shigemi, Department of Surgery, University of Maryland at Baltimore and Department of Biomedical Engineering, Johns Hopkins University

We investigated carotid sinus baroreflex control of vascular capacitance in $1 \mathrm{~K}-1 \mathrm{C}$ hypertensive and sham operated anesthetized dogs using $a$ constant flow cardiopulmonary bypass. Arterial compliance was a nonlinear function of arterial pressure and not different in hypertensive dogs. Carotid baroreflex-induced changes in arterial compliance, total peripheral resistance and zero-flow arterial pressure were not diminished in the hypertensive group. There were no significant differences in total systemic, arterial and venous compliances found between normotensive and hypertensive groups. In both groups, $13 \mathrm{ml} / \mathrm{kg}$ was mobilized by reflex venoconstriction, and $5 \mathrm{ml} / \mathrm{kg}$ of this was taken up by the arterial compartment, resulting in $8 \mathrm{ml} / \mathrm{kg}$ of blood mobilized to the reservoir. There were equivalent reflex changes in capacity in the normotensive and hypertensive groups, indicating that the ability to reflexly mobilize blood volume is not diminished in the face of the elevated arterial pressures in hypertension.

(Supported by NIH Grant HL-38316). 
67

THEORETICAL ADAPTATIONS OF THE SYSTEMIC CIRCULATION DURING EXERCISE

S. Magder and A. Deschamps. Critical Care Division, Royal Victoria Hospital, McGill University, Montreal, Canada.

During exercise, adaptations must occur in the systemic circulation to provide the venous return necessary to maintain a high cardiac output. Based on the two-compartment model of Krogh, Caldinj et al (Circ. Res. 1974) developed an equation for venous return (VR) which is based on the distribution of flow $(F)$, stressed vascular volume (V), venous resistance $(\mathrm{Rv})$ and venous compliance (Cv) in the splanchnic and peripheral circulations. We used values for these parameters that we previously obtained from canine studies on the hemodynamic effects of baroreceptor tone, heat stress and the infusion of neuropeptide $Y$ to predict changes in the circulation which would occur during exercise in a $70 \mathrm{~kg}$ man. An increase in the $F$ to the peripheral bed from $63 \%$ at rest to $90 \%$ during exercise would increase VR at zero right atrial pressure to $10.7 \mathrm{l} / \mathrm{min}$ from a resting value of $5.5 \mathrm{l} / \mathrm{min}$. If $\mathrm{V}$ also increased by $10 \mathrm{ml} / \mathrm{kg}$, VR would rise to $17.5 \mathrm{l} / \mathrm{min}$, but the pressure in muscle capillaries would rise considerably. If the venous resistance of the periphery decreased by $50 \%$, Q would increase further to $24.5 \mathrm{l} / \mathrm{min}$, and the rise in muscle capillary pressure would be less but still calculated to be 44 $\mathrm{mmHg}$. A decrease in the splanchnic Rv would increase VR to $28.6 \mathrm{l} / \mathrm{min}$ and produce a further rise in the peripheral capillary pressures. In conclusion, complex adjustments must occur in the systemic circuit to allow the high cardiac output during exercise. The high flow to muscles increases muscle capillary pressures which must be reduced by muscle contractions.

\section{8}

A NEW HEMODYNAMIC INTERPRETATION OF THE MUSCLE PUMP

John $V$. Tyberg and Sandra E. Baker

The University of Calgary, Calgany, Alberta

Evidence suggests that the circulatory phenomenon of exercise known as the muscle pump affects systemic capacitance (CAP) as well as systemic vascular resistance (SVR). However, it is difficult to measure these effects independently; indeed, to develop a conceptual framework to provide the basis of such an analysis.

When there is no flow through the systemic circulation, arterial pressure $\left(P_{2}\right)$ and venous pressure $\left(\boldsymbol{P}_{\mathrm{v}}\right)$ must be equal and, by definition, equal to mean circulatory pressure $\left(P_{m c}\right)$. $P_{m c}$ is a measure of the "fullness of the circulation" and reflects total CAP. As Lew and others have shown, increasing flow through the systemic circulation increases $P_{a}$ and decreases $P_{v}$ i these $P_{z}$ - and $P_{v}$-flow relations may be taken as properties of the systemic circulation, since they are functions of arterial and venous CAP and SVR. Analysis of recent experimental evidence (Sheriff et al, 1993) suggests that exercise may displace this relation righward (ie, the same values of $P_{\text {, and }} P_{*}$ appear to occur at higher flow values during exercise).

This result suggests a new hemodynamic model of the circulation during exercise. The exercising muscle may be equated to a pump connected between the aorta and the vena cava which pumps blood out of the arteries into the veins. Since this pump would be in parallel to the heart (obviously, the exercising muscle is also situated in parallel to the gut, kidney, etc), the pump flow would negate part of the cardiac output so plots of $P_{a}$ and $P_{v}$ versus cardiac output might be "artefactually" shifted rightward because flow through the non-exercising systemic circulation equals cardiac output minus musclepump flow. Correction for such a horizontal shift might make it easier to estimate the true change in $P_{m c}$ (by extrapolation of the $P_{a}-$ and $P_{v}$-flow curves to flow $=0$ ) and thereby, the effect of exercise on CAP.

69

EVAUUATION OF A FLW-DIFFERENTIN TECHNQUE POR THE RECORDING OF RECTONAL BLOOD VOUUMECHANGES.

J. R. McNeill and F.X. Ramapandian

Department of Pharmacology and Cardiovascular Risk Factor Reduction Unit, University of Saskatchewan, Saskatoon, SK. Canada, S7N OWO.

Baroreflexes play a major role in the regulation of regional blood volume changes but, unfortunately, anestherics and surgical stress alter baroreflex function. Therefore, we sough to develop and evaluate a method for the recording of regional blood volume changes in the conscious unrestrained animal by recording the integral of the difference between the inflow (arterial blood flow) and the outflow (venous blood flow) to a particular region using ultrasonic transit-time flowmeters (Transonic Systems, Ithaca, NY). As a first step, we attempted to validate the technique in the anesthetized cat by comparing the changes of splenic blood volume using the flow-differential technique to that recorded by an independen technique, namely a gravimetric method in which splenic weight was recorded by placing the organ in a cradle attached to a strain gauge transducer. Intra-arterial and intravenous infusions of norepinephrine were associated with dose-dependent decreases in splenic blood volume while isoproterenol induced increases in splenic blood volume. The peak changes recorded by the two methods at various doses of the two vasoactive agents showed a high degree of correlation $(r>.9)$. Moreover, the time course of the change of a single response using the flow-differential method was similar to that recorded by the gravimetric technique. The results suggest that the flow-differential method is a reliable method for the continuous recording of changes in spleen volume. However, in the conscious cat, the re-establishment of a collateral circulation to the spleen was a major problem limiting the usefulness of this approach, at least for this organ in the conscious animal. Other regions may not face the same revascularization problem. (Support by the MRC and the Heart and Stroke Foundation)
70

VASOCONSTRICTOR AGENTS AND MEAN CIRCULATORY FILLING PRESSURE Pang, C. C. Y.

Dept. Phamacol. \& Ther., The Univ. of British Columbia, Vancouver, B.C. V6T 123, Canada

We have used the mean circulatory filling pressure (MCFP) method to study the effects of drugs on venous tone in conscious, unrestrained rats. MCFP is the circulatory pressure that occurs after blood flow is instantaneously stopped and all the pressures are brought to an equilibrium. We investigated if central venous pressure (CVP) and portal venous pressure (PVP) equilibrate after the circulation is stopped by the inflation of a balloon implanted in the right atrium. In conscious rats, CVP was similar to PVP during circulatory arrest under conditions of normal, as well CVP was similar to PVP during circulatory arrest under conditions of normal, as well as drug-induced, elevated or reduced vascular tones. Pressor doses of vasopressin
$\left(5 \times 10^{-11}\right.$ to $1 \times 10^{-9} \mathrm{~mol} \mathrm{~kg}^{-1} \mathrm{~min}^{-1}$, i.v. infused) and $\mathrm{N}^{G}$-nitro-L-anginine methyl ( $5 \times 10^{-14}$ to $1 \times 10^{-9} \mathrm{~mol}_{\mathrm{kg}^{-1}} \mathrm{~min}^{-1}$, i.v. infused) and $\mathrm{N}^{G}$-nitro-L-arginine methyl
ester (nitric oxide synthase inhibitor, $2 \times 10^{-6}$ to $6 \times 10^{-5} \mathrm{~mol} \mathrm{~kg}$, i.v. injected) have minimal effects on MCFP. I.v. infused norepinephrine $\left(3 \times 10^{-10}\right.$ to $8 \times 10^{-9} \mathrm{~mol}^{-1} \mathrm{~kg}^{-1}$ $\left.\mathrm{min}^{-1}\right)$ and angiotensin $\|\left(2 \times 10^{-10}\right.$ to $\left.1 \times 10^{-8} \mathrm{~mol} \mathrm{~kg}^{-1} \mathrm{~min}^{-1}\right)$ dose-dependently elevated mean arterial pressure (MAP) and MCFP, part of angiotensin's venous effect was mediated via the release of catecholamines from the sympathetic nerve ect was mediat vil terminals. Endothelin-1 $\left(8 \times 10^{-11}\right.$ to $2 \times 10^{-9} \mathrm{~mol} \mathrm{~kg}^{-1}$. i.v. injected $)$ dose dependently increased MAP, but it only slightly raised MCFP in the resting condition, however, its venous effect was markedly potentiated by sympathetic activation (verapamil-induced hypotension). The MCFP method provides a reproducible and non-traumatic means for the estimation of drug effects on venous tone.

Supported by the Heart and Stroke Foundation of Canada.

71

ROLF OF GASTROINTESTINAL BED VASCULATUAE DURING MEASUREMENT OF MEAN CIRCULATOAY FILLING PAESSURE.

C. F. Rothe and R. Maass-Moreno.

Dept. Physiology, Indlana University School of Medicine, Indianapolis Indiana

The gastrointestinal (Gl) vascular responses to hemorrhage, transfusion (10 $\mathrm{ml} / \mathrm{kg}$ ) and sympathetic blockade by hexamethonium plus atropine (H\&A) was studied in fentanyl-pentobarbital anesthetized dogs. The Gl vascular compliance (Cgi) averaged $1.82 \pm 0.68$ (SD) $\mathrm{ml} / \mathrm{mmHg}$ and was not significantly changed by transfusion or hemorrhage, but sympathetic blockade increased Cgi $0.57 \pm 0.37$ $\mathrm{ml} / \mathrm{mmHg}$. During the mean circulatory filling pressure (Pmcf) maneuver the portal venous pressure (Ppv), assumed to be equal to the Pcv $7 \mathrm{sec}$ after stopping the heart, averaged $0.83 \pm 0.10 \mathrm{mmHg}$ higher than the Pcv. Furthermore, at this time the portal venous flow ( $F p v$ decreased to a plateau value about $25 \%$ of the pre-Pmcf flow. The Ppv-Pcv difference and Fpv-at-7-sec were not appreciably changed by transfusion, hemorrhage or reflex blockade. The product: Cgi ${ }^{\star}(\mathrm{Ppv}-\mathrm{Pcv})$, an estimate of the extra distending volume, was $1.3 \pm 1.2 \mathrm{ml}$, or about $0.07 \pm 0.07$ $\mathrm{ml} / \mathrm{kg}$ BW. From this source, the error in estimating Pmci, though finite, is physiologically insignificant. Some extra vascular distension of the liver during the Pmcf maneuver is possible, but unlikely if the normal distending pressure of the hepatic veins is appreciably less than the portal venous pressure.

(Research supported by NiH grant HL07723.)

\section{2}

ROLE OF CRITICAL CLOSING PRESSURE AND ARTERLAL RESISTANCE IN THE CONTROL OF THE CANINE VASCULATURE. S. Magder and Ian Shrier. Critical Care Division, Royal Victoria Hospital, McGill University, Montreal, Canada.

In most vascular beds, arterial flow stops even though arterial pressure is greater than venous pressure. This can be explained by the presence of arterial compliance and a vascular waterfall effect the critical closing pressure (Pcrit) at the level of the arterioles. We eliminated compliance effects by ramping flow to zero over $2-10 \mathrm{sec}$. and studied the effects of various physiological factors on Pcrit and the upstream resistance ( $\mathrm{Ra}$ ). We found that baroreceptor mediated increases in sympathetic activity, increased both Pcrit and Ra. Increases in the perfusion pressure increased Pcrit which suggests that there is a myogenic effect at the site of Pcrit. However, the arterial resistance decreased. Blockers of EDRF (L-nitro arginine) increased Pcrit and $\mathrm{Ra}$ and also the myogenic response, but the same pattern occurred with phenylephrine, an $\alpha$-agonist. Both agents eliminated the fall in $\mathrm{Ra}$ with increases in perfusion pressure. Maximal vasodilatation markedly decreased Pcrit, but it was still present (approx. 10 $\mathrm{mmHg}$ ); the myogenic response was also present but markedly reduced. A calcium channel blocker, nifedipine, reduced Pcrit but did not eliminate it. In conclusion, the pressure and flow of the hindlimb are controlled by alterations in both a Pcrit and $\mathrm{Ra}$, and the changes do not necessarily occur in the same direction. This allows tight regulation of hindlimb blood flow. 
73

\section{AN OPTIMIZED INDEX OF HUMAN CARDIOVASCULAR ADAPTATION TO SIMULATED WEIGHTLESSNESS \\ Mao Wang, Larry Hassebrook, Joyce Evans and Charles Knapp \\ Center for Biomedical Engineering, and Department of Electrical Engineering University of Kentucky, Lexington, KY 40506}

Prolonged exposure to weightlessness is known to produce a variety of cardiovascular changes some of which may influence the astronaut's performance during a mission. In order to find a reliable indicator of cardiovascular adaptation to weightlessness, we analyzed data from 9 male subjects after a period of simulated weighlessness produced by 2 hours in a launch position followed by 20 hours of $6^{\circ}$ head down tijt plus phamacologically induced diuresis with furosemide. Heart rate, arterial pressure, thoracic fluid index, and radial flow were analyzed. Autoregressive spectral estimation was used to estimate the spectra of each variable for pre and post simulated weightlessness. We found a significant decrease in heart rate power and an increase in thoracic fluid index power in the high frequency region $(0.2$ to $0.45 \mathrm{~Hz})$ and significant increases in radial flow and arterial pressure powers in the low frequency region $(<0.2 \mathrm{~Hz})$ in response to simulated weightlessness. However, due to the variability among subjects, no single variable provided a reliable measure for every subject. The backward elimination algorithm was then used to select the best discriminatory features from these spectral components and Fisher's linear discriminant and Bayes quadratic discriminant were used to combine the selected features to obtain an optimal index of adaptation to the exposure to simulated weightessness. Results showed that both techniques provided improved discriminant performance over any single variable and thus have the potential for use as an index to track adaptation and prescribe countermeasures to the effects of weightlessness. (Supported by NASA Grant NAG9-298, and the University of Kentucky Clinical Research Center Grant R01-2602.)

\section{4}

ANALYSIS OF MECHANISMS UNDERLYING THE CARDIOVASCULAR RESPONSE TO LOWER BODY NEGATIVE PRESSURE USING A MATHEMATICAL MODEL

E.H. Karam, R.S. Srinivasan, and J.B. Charles

Space Biomedical Research Institute and KRUG Life Sciences

NASA Johnson Space Center, Houston, TX 77058

This paper discusses a possible mechanism underlying the cardiovascular response to lower body negative pressure (LBNP) up to presyncopal levels. The analysis is based on simulation results obtained using a mathematical model of the cardiovascular system capable of accurately simulating LBNP response up to $-40 \mathrm{mmHg}$. In order to explain the response over a wider range of LBNP, the following hypothesis is made: The dynamics associated with left ventricular filling, which involve flow through pulmonary circulation and left atria, will change significantly as more and more blood is shifted away from the central region toward lower extremities during LBNP. This hypothesis was tested by comparing model's responses with and without changing the filling time constant. Experimentally observed presyncopal response could be obtained only with an inereased time constant at higher levels of LBNP. Although the actual process which determines the change of the filling time constant is not yet identified, the results indicate a high sensitivity of the response to increased time constant, which stems from its influence on the nonlinear left ventricular function. With the filling pressure already decreased due to decreased venous return caused by LBNP, an increased filling time constant further reduces the end-diastolic pressure. This reduction, although small, produces a significant drop in end-diastolic volume with consequent increase in heart rate and a sharp decline in mean arterial pressure at higher levels of LBNP. Thus, the model suggests that the presyncopal response is due, at least in part, to a leftward shift of the operating point on the passive left ventricular pressure-volume curve caused by the increased filling time constant during LBNP.

75

\section{DOES CONTROLLED RESPIRATION ENHANCE VAGAL MODULATION} OF HEART RATE ?

"Patwardhan, A.R., "J.M. Evans, 'D.L. Eckberg, and "C.F. Knapp.

*Center for Biomedical Engineering, University of Kentucky, Lexington, Kentucky, and

'Hunter Holmes McGuire V.A. Medical Center, Richmond, Virginia.

Breathing to a metronome has been reported to increase high frequency (HF) power in heart rate (HR) spectra, suggesting enhanced vagal modulation of HR [Malliani et al. Circulation, 84:482492, 1991]. To investigate the effects of controlled breathing on HR spectra we recorded ECG and respiration (nose thermistor) during supine (SUP), $50^{\circ}$ head up tilt (HUT) and $48 \mathrm{~mm} \mathrm{Hg}$ lower body negative pressure (LBNP). The tests were conducted during spontaneous breathing and controlled breathing, with the metronome set at each subject's spontaneous mean respiratory rate measured previously during SUP, HUT and LBNP. Breath durations were computed from the nose thermistor signal and probability density estimates for spontaneous breathing frequencies were estimated. Spectral estimates were computed using autoregressive (AR) models and wer decomposed to give components of the spectrum. The HF power in HR was quantified as the sum of powers of all the components within the respiratory frequency bandwidth rather than using the power of the largest component within the respiratory bandwidth. Results $(\mathrm{N}=8)$ showed no significant differences in either LF or HF powers between spontaneous and metronomic breathing for SUP, HUT or LBNP. Thus, when the variability in respiratory frequencies during spontaneous respiration was accounted for by summing all the spectral power in the respiratory bandwidth, HF powers were similar for spontaneous and metronomic breathing. Our results suggest that increased HF power in HR observed by others could be because, during controlled breathing all the respiratory components are coherent. This does not necessarily indicate enhanced vagal modulation of $H R$.
76

TIME-FREQUENCY AND SPECTRAL REPRESENTATIONS OF HEART RATE VARIABILITY OF SPACE SHUTTLE CREW DURING RE-ENTRY AND LANDING. Patwardhan, A.R., S. Vallurupalli, J.M. Evans, C.F. Knapp, "M.M. Jones, and "J.B. Charles. Center for Biomedical Engineering, University of Kentucky, and "Cardiovaseular Laboratory, JSC, NASA Houston TX.

To investigate whether the autonomic function in cardiovascular regulation changes due to microgravity exposure, and because heart rate spectra have been shown to indicate changes in autonomic function, we conducted spectral and time frequency analysis of heart rate from 11 astronauts before, during and after 5 to 6 day long spaceflight. Surface ECG was recorded for 5 to 6 minutes in the sitting (SIT) and 5 to 6 minutes in the standing position (STND) approximately 10 days preflight (L-10) and one hour after landing (LND). ECG was also recorded continuously during the last 5 to 6 minutes in microgravity environment, through re-entry, landing and subsequent egress. Instantaneous heart rates were computed from the ECG. Spectral estimates using autoregressive models were computed for SIT and STND position and from a 5 to 6 minute data record acquired in microgravity $(0 \mathrm{~g})$. The models were decomposed to obtain components of the spectra, which were binned into low (LF), mid (MF) and high (HF) frequency regions (.01-.06, .06-.15, and .15-.5 Hz). To track changes in heart rate variability during re-entry and landing (i.e. during the transition from inicrogravity to earths $1 \mathrm{~g}$ environment) we conducted a non-stationary time-frequency analysis using Smoothed Discrete Wigner Distribution (SDWD). In the SIT posture, (LF+MF)/HF powers during $\mathrm{L}-10$ and $0 \mathrm{~g}$ were similar but were significantly elevated during $\mathrm{LND}$. The total powers in heart rate spectra were elevated during $0 \mathrm{~g}$ relative to $\mathrm{L}-10$ and $\mathrm{LND}$. The orthostatic response (decrease in $\mathrm{HF}$ and $a$ increase in $L F$ and MF powers) was blunted during $L N D$ relative to that during $L-10$. The time-frequency analysis showed a increase in LF and MF and decrease in $\mathrm{HF}$ powers during landing. These spectral changes suggest that the cardiovascular regulatory system re-adjusts after 5 to 6 days in microgravity such that during $0 \mathrm{~g}$ the autonomic balance is similar to that during $\mathrm{L}-10$, but after re-entry, the balance shifts towards increased sympathetic and decreased parasympathetic activity.

77

COMPARATIVE HEMODYNAMICS: ALLOMETRIC ANALYSIS OF CARDIOVASCULAR VARIABLES

\section{J.K-J.Li, Y. Zhu, G. Drzewiecki and A. Noordergraaf}

Cardiovascular Research Labs., Biomedical Engineering, Rutgers University, NJ and Cardiovascular Studies Unit, Bioengineering, University of Pennsylvania, PA.

Hemodynamic variables that are commonly used to assess cardiac function and the arterial system are either directly measured, or derived from models. Some of these parameters vary considerably among mammals of differing body sizes. For comparative analysis, normalization process is often necessary. We investigated this aspect by applying allometry to a generalized left ventricle-arterial system model. Results show that pulse transmission parameters such as pulse wave velocity and reflection coefficient, aortic propagation constant times length, are independent of body sizes. This is also true for ratios of geometric dimensions, such as arterial and ventricular wall thickness to radius ratios. Stroke volume and arterial compliance are proportional to body weights and ventricular elastance is inversely proportional to cardiac muscle volume. Normalization is then simply obtained based on these relations. Allometry thus provides a powerful tool for comparative hemodynamic studies.

78

POST-PRANDIAL HEART RATE SFECTRAL CHANGES IN YOUNG NORMAI S.

Tushar Desai, Jerry Collins, Richard Shiavi, Italo Biaggioai, and David Robertson. Vanderbilt University, Depts. of Biomedical Engineering and Medicine.

The effects of a standard meal on heart rate (HR) spectra and systemic blood pressure (BP) was evaluated in 20 young normals ( $10 \mathrm{M}$ and $10 \mathrm{~F}$; ages 20-29). After abstaining from caffeine for $24 \mathrm{hrs}$ and an overnight fast, 8 subjects (4 M, 4 F) were asked to lie supine for $60 \mathrm{~min}$ before meal and 60 min after. A second group of $12(6 \mathrm{M}, 6 \mathrm{~F})$ lay supine for $30 \mathrm{~min}$ before and $90 \mathrm{~min}$ after a meal. While the subjects were supine, ECG was recorded continuously and BP was measured at 5-min intervals. For each 10-min interval, a smoothed time series of instantaneous HR was generated, on which Welch's method of averaging modified periodogram was used to calculate power spectre in the range of 0.02 to $0.4 \mathrm{~Hz}$. Spectral power was divided into two bands-low-frequency (Lf, $<0.15 \mathrm{~Hz}$ ) reflecting primarily sympathetic control of HR and high-frequency $(\mathrm{Hf},>0.15 \mathrm{~Hz}$ ) reflecting vagal control of HR. No significant postmeal and high-frequency (Hf, $>$ ofences were found in BP between genders or over either the 60-min or 90-min intervals by differences were found in BP between genders or over either the 60-min or 90-min intervals by
repeated-measures ANOVA. While females had significantly higher average premeal HR, postmeal HR showed significant increases for both sexes, with the most change occurring in the first $60 \mathrm{~min}(60 \pm 8 \mathrm{bpm}$ to $66 \pm 8 \mathrm{bpm}, \mathrm{n}=20, \mathrm{p}<0.0001)$ and persisting over $90 \min (62 \pm 10$ bpm to $66 \pm 7 \mathrm{bpm}, n=12, p<0.0001$ ). The Lf/Hf matio also showed significant increases postmeal for both $60 \min (3.4 \pm 2.3$ to $4.4 \pm 2.2, n=20, p=0.005)$ and $90 \min (4.1 \pm 2.6$ to $5.0 \pm 3.1, n=12, p=0.0008)$ intervals. Contrast analysis was performed on the Lf/Hf ratio $5.0 \pm 3.1, n=12, p=0.0008)$ intervals. Contrast analysis was performed on the Lf/Hf ratio
averaged over 30 -min premeal and either two $(n=20)$ or three $(n=12)$ postmeal intervals. The $\mathrm{Lf} / \mathrm{Hf}$ ratio did not increase significantly for any two adjacent time intervals, but the increases between any two non-adjacent intervals (i.e. premeal to second or third 30 min postmeal or first $30 \mathrm{~min}$ to third $30 \mathrm{~min}$ postmeal) were significant. Meals, therefore, caused increases in HR and Lf/Hf ratio in normal subjects persisting over a 90-min postmeal interval, consistent with an increase in sympathetic autonomic HR control at the expense of vagal control. 
79

\author{
APPAOACHES TO HIGH-LEVEL EXPRESSION OF HEMOGLOBIN IN \\ TRANSGENIC PIGS" \\ R. Kumar, A. Sharma, A. Khoury-Christianson, J. Okabe, and M. Martin \\ DNX, Princeton, NJ
}

The production of transgenic pigs expressing integrated hemoglobin transgenes (Swanson ot. al. Bio-Technolegy 10:557-559, 1991) has been heralded as a breakthrough in the development of a cost-effective and safe hemoglobin based oxygen carrier (Moffat, Science, 253:32-33, 1991). The cost of hemoglobin will be determined in large part by the level of expression of the human globin transgenes in the transgenic pigs. We have initiated a multi-faceted effort to optimize the elficiency of production of human hemoglobin in transgenic pigs. We have addressed both the transcriptional and translational control aspects of gene regulation in the design of constructs that allow high-level expression of human regulation in the design of constructs that allow high-level expression of human globin genes in pigs. Other aspects of protein synthesis and assembly, such as folding and sub-unit association are also being considered in our design. The results of these experiments and their impact on the bio-engineering of a blood
substitute will be discussed.

80

KINETICS OF NADH-METHEMOGLOBIN REDUCTASE IN LIPOSOMEENCAPSULATED HEMOGLOBIN

S. Zheng, D. Webster and R. Beissinger, Illinois Institute of Technology, Chicago, Il

Modified film hydration and double emulsion approaches resulting in improved encapsulation efficiency have been developed for encapsulation of hemoglobin in lipid vesicles for use as a red blood cell substitute. Recent work has demonstrated using either of these approaches that administration of liposome-encapsulated hemoglobin (LEH) in rats was efficacious. The importance of inhibition of the generation of methemoglobin (metHb; the nonfunctional form of hemoglobin which cannot carry oxygen) during the processing of LEH is demonstrated in our current studies. The enzymatic activity of metHb reductase as well as its effect on methlb concentration in precursor Hb solution compared to liposomeencapsulated $\mathrm{Hb}$ solution was measured spectrophotometrically. The enzyme kinetics were studied using metHb as an electron acceptor. NADH was used as the enzyme cofactor. Stroma-free Hb solution was used as the crude enzyme source of metHb reductase, which was encapsulated in lipid vesicles. Results showed for stroma-free $\mathrm{Hb}$ solution stored for up to 20 months at $-20^{\circ} \mathrm{C}$ that the enzyme system was active (specific activity $=0.54 \mu \mathrm{mol}$ metHb/min-mg total protein). Encapsulation of this $\mathrm{Hb}$ solution in liposomes using modified film hydration processing to form the LEH resulted in somewhat reduced enzymatic activity $(0.36 \mu \mathrm{mol}$ metHb/min-mg total protein). The effect of NADH-metHb reductase on metHb reduction in LEH samples was then studied. The metHb concentration of the liposomeencapsulated $\mathrm{Hb}$ solution, which was formed using modified film bydration processing, was $9.5 \%$ of the total $\mathrm{Hb}$ (without NADH added to the precursor $\mathrm{Hb}$ solvtion) and more than $50 \%$ less, i.e. $4.5 \%$ (with NADH), both at day 2 , respectively; metHb was $13.2 \%$ (without NADH) and $5.6 \%$ (with NADH) at day 15 . However, LEH made using either reverse evaporation or double emulsion processing, both of which included the use of organic solvents, showed no significant reduction in the metHb concentration of the encapsulated $\mathrm{Hb}$ solution for LEH sample containing NADH compared to that without NADH.

81

CARDIOVASCULAR EFFECTS OF BEMOGLOBIN SOLUTIONS:

CHARACTERIZATION AND MECHANISMS

T. Estep, Baxter Healthcare Corporation, Round Lake, IL

In-vivo testing has demonstrated that highly purified hemoglobin solutions exhibit cardiovascular effects which are not explicable simply on the basis of oxygen transport or colloidal properties. Of particular interest is a rapid increase in blood pressure which is observed in both normovolemic and bypovolemic animals immediately after hemoglobin infusion. This pressor response is self-limiting in that the blood pressure increase does not exceed 40 to $60 \%$ of baseline values in normotensive animals, regardless of the dose of hemoglobin administered. While associated with an increase in total peripheral resistance, the administration of hemoglobin results in increased blood flow to many organs, especially in hypovolemic animals. Studies of potential mechanisms for this organs, especially in hypovolemic animals. Studies of potential mechanisms for this
cardiovascular response implies that hemoglobin interacts with endothelin, $\alpha$-adrenergic and nitric oxide pathways. Although the precise interrelationship between these interactions is unclear, the net result is a redistribution and selective enhancement of blood flow which is beneficial in several preclinical models of important human indications.
82

THE EXPRESSION OF TUMOR NECROSIS FACTOR BY MACROPHAGES EXPOSED TO LIPOSOME ENCAPSULATED HEMOGLOBIN

Alan S. Rudolph ${ }^{1}$, Richard O. Cliff, Andreas M. Spirig ${ }^{3}$, and Rueven Rabinovici ${ }^{3}{ }^{1}$ NAVAL RESEARCH Laboratory, Code 6900, Washington, DC 20375-5348, ${ }^{2}$ Geo-Centers, Inc., 10903 Indian Head Highway, Ft. Washington, MD 20744, ${ }^{3}$ Department of Surgery, Thomas Jefferson University, Philadelphia, PA 19107-5083

We have demonstrated that the encapsulation of hemoglobin results in biodistributions which show significant involvement of the reticuloendothelial system. Previous studies with moderate doses of radiolabeled liposome encapsulated hemoglobin (LEH) in small animals have demonstrated that the principal site of accumulation is tissue resident macrophages of the liver and spleen over the course of $24-48$ hours. Histopathology of the liver and spleen after injection of LEH shows enlarged Kuppfer cells and trapping of LEH in the spleen. Based on these findings, we have explored the physical and biochemical responses of cultured and explanted macrophages with LEH. Murine alveolar, peritoneal, and splenic macrophages as well as adherent human peripheral blood monocytes have been incubated with LEH and the expression of tumor necrosis factor (INF) measured by ELISA and by a cell based $L 929$ kill assay. The results indicate that macrophages do not express TNF when incubated with varying concentration of LEH for up to 12 hours. We have also examined the ability of cultured explanted macrophages to express TNF in response to an endotoxin challenge following incubation with LEH. Macrophages exposed to LEH for 12 hours and then challenged with LPS for 24 hours show reduced expression of TNF compared to macrophages exposed to LPS alone. This is in contrast to increased serum TNF levels measured in rats injected with LEH followed 90 minutes later with a bolus injection of LPS. We are currently exanining the time course of these effects and this response in ex vivo macrophages from mice injected with LEH. These results will also be presented in the context of possible direct interactions between endotoxin and components of LEH.

83

MECHANISMS OF HEMOGLOBIN TOXICITY

R.M. Winslow, MD, K.D. Vandegriff, PhD, and R. Motterlini, PhD

Department of Medicine, School of Medicine, and Department of Bioengineering (AMES), University of California, San Diego, 3350 La Jolla Village Drive, San Diego, CA 92161.

Several cell-free hemoglobin products, modified to prolong vascular retention and to minimize renal toxicity, are now in buman safety trials. While some of the problems with early solutions have been overcome by rigorous purification and better product characterizations, problems still remain. Chief among these is vasoactivity, possibly mediated by the known property of hemoglobin to bind NO, an endothelium-derived relaxing factor. Altematively, NO might be destroyed by $\mathrm{O}_{2}$ radicals formed in the presence of hemoglobin. This obstacle has challenged our understanding of endothelial cell biology, of certain fundamental properties of hemoglobin, such as the heme-globin linkage, and of the pathways of heme metabolism. Some chemical modifications of bemoglobin lead to a relative loosening of the heme-globin linkage, and free heme can also bind NO. Heme can be taken up by endothelial cells in culture, as demonstrated by stimulation of heme oxygenase. It is possible that a better understanding of the globinheme linkage and the effects of modification on it might lead to safer products in the future. Altematively, encapsulation of hemoglobin into liposomes could limit hemeendothelium interaction and could provide additional safety, provided toxicity from liposomes is minimal. Finally, increasing the molecular size of hemoglobin by polymerization or conjugation to another molecule might reduce interstitial hemoglobin "leak", and could provide increased safety, provided significant globin-heme dissociation does not occur. 
85

DEVELOPMENT OF A PULSATLE, VENTRICULAR ASSIST DEVICE FOR INFANTS Juretich, J., Holfert, J., Bums, G., Taylor, T. Pantalos, G., Koppert E. Olsen, D.

University of Ulah, Anificial Heart Research Laboratory.

The infant patient population has been among the fastest growing segments of cardiac surgery over the past ten years. Increasing numbers of corrective procedures and transplantations have been successfully accomplished at a growing number of centers. As the number of cardiovascular interventions grow, and as the field expands, the number of complications associated with these surgeries are bound to increase accordingly. Presently, there is no reasonable option available for temporary mechanical circulatory support for the infant popularion.

In response to this need, the University of Utah's Artificial Heart Research Lab has been developing a $10 c c$ infant ventricular assist device (IVAD). This device is an extracorporeal, pneumatically powered ventricle which can be used for either single or biventricular support.

Design and development of the device over the past two years has focused primarily on the following four areas: 1) ventricle design, 2) valve design, 3) cannulae design, and 3) driver design. Each area is being evaluated for applicability to the anticipated patient population. Considerations for the ventricular design have included cardiac output, flow pattem characteristics, manufacturability, reliability and functionality. Vaive design parameters include minimizing thrombosis and hemolysis, simplicity, manufacturability, reliability, and assembly consideration. Cannuiae design is focusing on matching flow requirements with patient vessel size limitations and the resulting effect on blood interacrions. The driver development is currently underway, and determination of control parameters, conorol modes, alarm systems, etc. are being

The IVAD system configuration and design integration has focused on simplicity for use in a clinical setting. Ease of implantation, monitoring, and evaluation will all be factors in assessing the usefullness of the IVAD as a safe and effect tool in the treatment of patients with cardiac illness in the infant population.

86

SCALE EFFECTS IN THE DESIGN OF A PULSATTLE VENTRICULAR ASSIST DEVICE (VAD) FOR INFANT

S. P. Sutera, B. B. Daily and T. W. Pettitt

Washington University, St. Louis

Steady flows of an incompressible viscous fluid through two geometrically similar regions are dynamically similar, i.e., have the same relative magnitudes of inertial and viscous forces, if the Reynolds numbers (Re) are the same. If the flows are transient or periodic, Re equality is not by itself a sufficient condition for dynamic similitude. In such flows the transient growth of viscous boundary layers, wakes, and regions of flow separation and stasis must also be reckoned. A pulsatile VAD designed to be geometrically similar to an existing device for adult patients, but scaled down to give a stroke volume of $-10 \mathrm{cc}$, will have a length scale $\sim 1 / 2$ that of the adult prototype. The limited availability of valve types and sizes will generally preclude geometrically similar scaling of inflow and outflow ports. This reality will complicate the determination of corresponding velocity scales as will the controlled variability of beat frequency. In general it must be expected that the flow in a scaled down VAD will have a lower $\mathrm{Re}$ indicating a greater influence of viscous effects and also boundary layers that are thicker relative to the flow passages. The magnitude of wall shear stresses inside the smaller ventricle can be comparable to that of the adult-sized unit but will depend strongly on the size of the valve ports.

87

HYDRODYNAMIC PERFORMANCE OF A PEDIATRIC VENTRICULAR ASSIST DEVICE Timothy W. Pettitt, M.D., Salvatore P. Sutera, Ph.D., Bill B. Daily, M,D., Ph.D. Washington University, St. Louis, Missouri

Despite the success of ventricular assistance in selected adult patients and the clinical need for similar assistance in neonates and infants, no such pediatric device is currently available. The development of a pediatric ventricular assist device (PVAD) has been hindered by the lack of small, commercially available valves. A scaled down version of the Pierce-Donachy VAD, with specially designed ball valves, has been fabricated by Sarns $3 \mathrm{M}$, Inc. Animal studies, performed at The Pennsylvania State University, demonstrated a tendency for thrombus to form on the blood sac surface with peripheral microthromboembolism. Except for the valvular apparatus, the adut and pediatric devices are geometrically similar and made from the same materials. Therefore. we and peciate de of less effective washing of the sac surace. We suspect that the maximal wall shear stresses are substantially lower in the PVAD because of lower flow rates imparted by the smaller size of the device and restrictive nature of the small, hemodynamically inferior $6 \mathrm{~mm}$ ball valves. We tested the hydrodynamic peremance of this PVAD in viro mock circulation studies using two different valve/cannulae systems: (1) the original $6 \mathrm{~mm}$ inner diameter ball valves and (2) commercially available $12 \mathrm{~mm}$ porcine tissue valve conduits. Over a physiologic range of flows and pressures and for an operational range of drive parameters (beat rate, drive air pressure, systolic duration) we found the mean pressure gradients $(\Delta P)$ and energy losses $(\Delta E)$ were significantly reduced

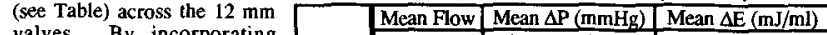
valves. By incorporating larger, hemodynamically superior valves, we expect to improve washing of the blood sac surface by increasing the kinetic energy of the inlet as it impinges on the blood sac bes on the blood sac surface.

\begin{tabular}{|c|c|cc|cc|} 
& $(\mathrm{ml} / \mathrm{min})$ & $6 \mathrm{~mm}$ & $12 \mathrm{~mm}$ & $6 \mathrm{~mm}$ & $12 \mathrm{~mm}$ \\
\hline \multirow{3}{*}{ Inlet } & 1000 & 7.36 & 2.34 & 1.04 & 0.35 \\
& 1500 & 14.94 & 3.72 & 2.10 & 0.56 \\
& 2000 & 28.10 & 5.10 & 4.04 & 0.78 \\
\hline \multirow{3}{*}{ Outlet } & 2000 & 16.88 & $\mathbf{8 . 0 2}$ & 2.75 & 1.43 \\
& 3000 & 34.45 & 13.51 & 5.43 & 2.26 \\
& 4000 & 58.67 & 19.00 & 9.26 & 3.08 \\
\hline
\end{tabular}

88

PEDIATRIC BLOOD PUMP DEVELOPMENT

Kenneth .C. Butler, Timothy .R. Maher, Harvey S. Borovetz, Robert L. Kormos and Bartley P. Griffith

Nimbus, Inc., Rancho Cordova, CA (KCB, TRM) and University of Pittsburgh (HSB, RLK, BPG)

One problem facing cardiac surgeons who operate on thousands of neonates and pediatric aged patients annually or who wish to provide pre-operative support for congerutal cardiac anomalies is lack of suitable devices to provide temporary cardiac support. Existing devices available for such purposes (ECMO and centrifugal pumps) are too large relative to the size of the patient. With this deficiency in mind Nimbus and the University of Pittsburgh (UOP) Department of Surgery are working to develop a new, miniaturized rotary pump intended solely for these special young patients. The current prototype generates 0.3 to $3.0 \mathrm{~L} / \mathrm{min}$ flow at a speed range of 2500 to 4000 RPMs. The calculated average index of hemolysis obtained for in vitro tests with bovine blood equals 0.27 grams/day which is a factor of 11 below reported results for currently available centrifugal pumps. The key innovation is that the Nimbus/UOP neonatal/pediatric pump is $1 / 5$ to $1 / 3$ the size of existing centrifugal pump with a very low priming volume of only $15 \mathrm{cc}$. The feasibility of this pump concept is being addressed through in vitro studies of flow performance, hemolysis generation and lip seal integrity. In vioo tests will demonstrate the hemodynamic performance, biocompatibilty and general suitability of this pump for neonates and pediatric aged patients. If ultimately successful, the Nimbus/UOP VAD would be the first product which is intended solely for cardiac support of neonates and pediatric aged patients.

89

THE PNEUMATIC PEDIATRIC VENTRICULAR ASSIST DEVICE-

PENN STATE UNTVERSITY ANIMAL, STUDIES

Sapirstein JS, Pae WE, Wirt DF, Marlotte JA, Pierce WS

Department of Surgery, The Pennsylvania State University and Sarns/3M, Inc.

Despite improvement in the results of pediatric cardiac surgery, certain patients develop inadequate cardiac output after operation. Because such procedures usually do not involve ischemic myocardiun, the potential for functional recovery from profound post-cardiotomy cardiogenic shock with mechanical ventricular support should be greater than observed in the adult population. The Pennsylvania State University and Sarns/3M have developed a scaled-down model of the Pierce-Donachy pneumatic assist device for use in the left or right ventricles of pediatric patients. An ultrasmooth, seamless $18 \mathrm{ml}$ blood-sac of segmented polyurethane is contained within a two-piece rigid housing. Air pulses from a pneumatic drive unit provide for filling and emptying, while umiquely designed ball valves assure unidirectional flow. A stroke volume of $12 \mathrm{cc}$ provides a maximum output of $1500 \mathrm{cc} / \mathrm{min}$, yielding appropriate flows of $100 \mathrm{cc} / \mathrm{kg} / \mathrm{min}$ for patients ranging from newbom to approximately six years of age. Short-term chronic experiments of teft ventricular assistance were conducted in 18 baby goats and 10 young calves. Pumping duration averaged 19 days (range $=1.29$ days). Mechanical failures occurred in $18 \%$, and thrombus formation associated with the pump or cannulae was noted in $64 \%$ of animals despite aggressive anti-platelet activation and heparin regimens. Embolic events were documented at autopsy in $18 \%$ of animals. The Pennsylvania State University is now planning further in vitro and animal studies to address these problems. 
91

BIOMEDICAL ENGINEERING CONSIDERATIONS FOR CHRONIC DEVICE PATIENTS

J.M. Pristas, S. Winowich, CJ. Capretta, R.L. Kormos, H.S. Borovetz

University of Pittsburgh Medical Center, Pittsburgh, PA 15213

As the cardiac transplant waiting time continues to increase, current generation mechanical circulatory support (MCS) systems are being used to support patients for longer periods of time. There are several biomedical engineering issues that must be addressed when designing the next version of these systems. Currently, at the University of Pittsburgh Medical Center (UPMC), a biomedical engineer (BME) is available in-hospital 24 hours a day to provide technical support to the medical and nursing staff. When the patient's condition permits, a training program is initiated to teach the patient and family about the support system. This teaching is essential to the progression to our out-of-hospital facility, Family House (FH). We intend to initially keep a BME on duty at FH, but slowly wean them from that position as the patient and family become more comfortable with the MCS system. Should a technical emergency arise, the patient and/or their significant other becomes directly responsible for implementing the proper emergency procedures. As the patient moves farther away from UPMC, their role becomes even more critical and training is more intense, and may be extended to include community hospitals. Other issues that our BME staff have discussed
ind extended to include community hospitals. Other issues that our BME staff have discussed
include system hardware components, such as display monitors, backup controllers and adequate battery support. The patient should carry a portable cellular telephone so that they can contact 911 or the medical/technical staff directly if they have a medical or technical problem. A BME may need to perform occasional system checks on the equipment to ensure continued safe operation. Alarm systems must be designed into the system to alert the patient, or perhaps a BME at UPMC, of conditions that require immediate attention. Messages on the device monitor should instruct the patient as to the resolution of the alarm. Also, as required by the FDA, data collection must be maintained, either by the patient, the MCS itself, or through remote monitoring via modem. All of these BME issues must be considered when designing the future chronic MCS systems.

92

DEVELOPMENT OF A WEARABLE CONTROLLER FOR THE NOVACOR LVAS P. Miller, J. Billich, D. LaForge, J. Lee, A. Naegeli, J. Jassawalla, P. Portner Novacor Division, Baxter Healthcare Corporation

The Novacor left ventricular assist system (LVAS) has been utilized in an ongoing clinical trial as a bridge to cardiac transplantation since 1984, using an implanted blood pump and electromechanical driver, and an extracorporeat control console. Since patients supported by the LVAS typically become rehabilitated and highly mobile, a wearable control system configured expressly for the ambulatory patient was developed. This controller was ergonomically designed for portability, convenience and appearance, offering the recipient improved mobility and enhanced self-image.

The wearable control system is comprised of a compact controller and rechargeable battery packs. The microprocessor-based controller provides all of the functions validated during 15 patient-years of clinical experience with the control console, including synchronous operation based on internal sensing of pump filling rate. The main battery, capable of supporting pump operation for up to seven hours, includes a "smart" monitoring circuit, providing a display of battery charge level and a two-stage low charge alarm. The smaller reserve battery provides a redundant source of power and supports pump operation while a depleted main battery is being replaced. A monitor unit, based on a portable PC-type computer, provides the operator interface for device monitoring and adjustment. It displays patient ECG and pump volume waveforms, along with numeric values of rate, stroke volume and output. The monitor also supplies power (from AC or internal battery) to the wearable controller in lieu of the main battery pack.

The wearable control system has been tested extensively in vitro and in vivo, with demonstrated multi-year reliability. A clinical trial of the system is currently underway.

93

IMPLANTED ROTARY BLOOD PUMPS - DEVELOPMENT CHALLENGES

K. Butler, T. Maher, H. Borovetz*, J. Antaki*

Nimbus, Inc. and *The University of Pittsburgh

A number of investigators, including ourselves, are developing rotary blood pump technology for implanted cardiac assist systems. Our motivation to pursue such devices stems from inherent advantages rotary pumps offer relative to existing pulsatile blood pumps. In particular, there is the potential to very significantly decrease the size, weight, complexity, and cost of implanted systems if rotary blood pumps having the necessary biocompatability, durability, and reliability characteristics can be developed. We think that indeed this is possible and that rotary blood pumps may evolve to the point where implanted heart assist systems become a widely used form of treatment for end-stage heart disease.

Along with those important advantages, rotary blood pumps have their own development challenges which must be met if our evolutionary vision is to be fulfilled. The main ones lie with (1) achieving blood seal integrity, (2) verifying pump reliability, (3) providing pump speed control, and (4) demonstrating long-term physiological compatibility of diminished pulse flow. The systems Nimbus and the University of Pittsburgh are jointly developing utilize axial flow blood pumps. We have sufficient in vitro and in vivo experience with these devices to indicate that our unique system concept, as well as our basic development approach, will succeed in effectively addressing all of those challenging issues.

\section{4}

PERCUTANEOUS ACCESS (PA) AND VENTRICULAR ASSIST DEVICES (VADs) John C. Woodard

Novacor Division, Baxter Healthcare Corporation

The current generation of ventricular assist devices are extemally powered or extracorporeal and therefore require chronic penetration of the integument for either power/control or vascular access. As VAD implant durations increase and device recipients begin to be cared for out of the hospital environment, the PA device and the clinical management of the exit site will out of the hospital environment, the PA device and the clinical man
become critically important for the survival of these VAD patients.

An ideal PA device would provide a durable seal at the skin surface functioning as impervious barrier to bacterial penetration while requiring little or no maintenance in the form of dressings or cleansing. Unfortunately, none of the current PA devices fulfill these criteria. The design of a number of these currenily-used VAD PA access devices will be reviewed and comparative photographs of the associated exit sites will be shown. Clinical experience gained from the Investigational Device Exemption trials of the Novacor Left Ventricular Assist System (LVAS) indicates that al though wound dehiscence may occur at the exit site and infection occurs in a small percentage of patients, infection has always been resolved and no life-threatening events have been precipitated.

A common feature of the Novacor LVAS and many other VAD PA devices is the use of a long transcutaneous cuff fabricated of low-pile Dacton velour. This is in contrast to the short, high pile subcutaneous Dacron cuffs on the Tenckhof Chronic Ambulatory Peritoneal Dialysis catheter. Other implants that cross external membranes such as dental implants and fixation posts for auricular prostheses utilize titanium rather than polymeric materials. Long survival and posts for auricular prostheses utilize titanium rather than polymeric materials. Long survival and improved by both design and material changes.

95

ONGOING DEVELOPMENT OF COMPLETELY IMPLANTED HEART ASSIST AND HEART REPLACEMENT SYSTEMS

A. J. Snyder, G. Rosenberg, W.J. Weiss, W.E. Pae, W.S. Pierce

The Pennsylvania State University

We have for over ten years been engaged in the development of completely implanted, long-term systems for assistance of the left heart and for orthotopic hear replacement. Both are based upon a smooth-surface sac type pump actuated by brushless motor and rollerscrew electromechanical energy converter. These pumping units have been successfully coupled with implantable electronic controls, and transcutaneous energy and telemetry subsystems, enabling tether-free, intact-skin
operation in experimental animals.

Our experience with animals indicates that the devices can function for extended periods without hemolysis requiring red cell replacement or clinically relevant changes in end-organ function. Although long-term durability cannot be demonstrated except through real-time testing, devices have repeatedly operatedc

Both systems are now reaching their final physical configurations, in preparation for durability procedures, long-term availability of materials and components, and human factors.

Human factors considerations weigh heavily in the design of the extracorporeal equipment (energy transmission primary package, battery package, and battery charger), but also affect other parts of the design, such as the required capacity of the implanted battery. We have drawn here upon our experience with patients undergoing bridge to transplant procedures with pneumatic devices. This experience indicates that the majority of patients are easily made to feel comfortable with the equipment, tend to be highly confident in their devices, understand the relative importance of various alarms and indicators without extensive training, and are eager for mobility. Thus, the system configuration requires that the user attend to it periodically (typically every four hours) and assumes
a modest amount of training in its use. In retum, the user is granted a high degree of mobility, and, we hope, a sense of normality.

96

CLINICAL EVALUATION OF THE CARDIOWEST C-70 TOTAL ARTIFICIAL HEART

R. G. Smith, MSEE, CCE

University Medical Center, Tucson, Arizona

The purpose of an Investigational Device Exemption (IDE) application is to collect specific information to support the safety and effectiveness of a device. This information includes system description, manufacturing, in vitro testing, in vivo testing, and data from the clinical investigation. The goal is for this data to support a premarket approval application (PMA).

The CardioWest C-70 Total Artificial Heart (TAH) system is similar to the old Symbion J7-70 TAH system. During the study design, having data on over 140 past bridgeto-transplant patients provided many advantages. For example, additional entrance criteria were based on limitations observed with previous patients. Study exclusion criteria now address small size, elevated cytotoxic antibody levels, currently on a ventricular assist device, and chronic steroid therapy, because all were associated with poor outcomes in the previous study. Data collection was structured to minimize different interpretations in a multicenter study. For example, "irreversible kidney function" as a contraindication to implant was replaced with "no dialysis for the past seven days and a serum creatinine less than or equal to five." Study success criteria and risk analysis were also based on previous human studies. Prospective controls and investigator bias were addressed in the new study. The learning experiences of past studies have allowed a more focused approach to the new clinical evaluation. This should minimize variability that would extend the study and require more data to meet PMA requirements. 
97

AN ENTERIC POUCH TRANSFORMER FOR CIRCULATORY SUPPORT DEVICES: ANALYSIS OF A NEW TRANSFORMER CONFIGURATION

A Helmicki, T Benderson, D Melvin, D Glos, D Nebrigic;

Colleges of Engineering and Medicine, University of Cincinnati.

Circulatory support devices for indefinite heart failure treatment require 10 to 20 watts of power. Transmission of this power via transcutaneous electrical induction was demonstrated over 30 years ago [Scuder et al, 1961]. Three such devices now approach requirements for over 30 years ago [Scuder et al, 1961]. Three such devices now approach requirements for
human use (Novacor, Thermedics, and Ottawa). All of these devices are air-cored with the
secondary implanted subcutaneously and the primary worn externally. In 1989 work began at the University of Cincinnati on a transcutaneous electrical induction device utilizing bigh permeability core material. This configuration offers significant advantages in two respects: First, the use of high permeability core material concentrates the magnetic field thereby resulting in greater efficiency of power transfer and less heat dissipation in surrounding tissues. Second, the use of high permeability core material allows the device dimensions to be reduced to the point where a novel method of implantation utilizing an isolated intestinal pouch is possible. This, in turn, helps to alleviate problerns with existing approaches in the areas of device positioning and alignment, and patient comfort due to tactile sensitivity of skin. A first generation device has been developed employing this principle. This device uses a high permeability cored primary mounted axially within a toroidal secondary. Preliminary test results are given in a companion paper appearing in this conference. The purpose of this paper is to report on the development of a second generation device which employs high permeability core materials in both the primary and secondary. This new configuration has resulted in even further increases in operating efficiency and decreases in device size. The specific research reported herein includes an analysis of this new configuration together with validation of this design based on: (i) simulation results obtained from computer modeling using the MSC/EMAS CAD package, and (ii) preliminary results of calorimetry studies of device energy transfer and heat loss.
98

AN ENTERIC POUCH POWER TRANSFORMER FOR CIRCULATORY SUPPORT DEVICES: HEAT DISSIPATION D Melvin, D Glos, D Nebrigic, A Helmicki, T Henderson, D Duplain, J Saikali, S Hunter; Colleges of Medicine and Engineering, University of Cincinnati.

Circulatory support devices for indefinite heart failure treatment require 10 to 20 watis of power. Transcutaneous wires or tubes risk infection while neither battery nor skeletal muscle power has to date been quantitatively sufficient. Effective transcutaneous electrical induction was demonstrated over 30 years ago[Scuder et al 1961] and three such devices now approach re destulated that power requirements for human use (Novacor, Thermedies and onta). We postion induction from an isolated intestinal pouch might have advantages in position stability, heat dissipation, comfort, and device size. Studies began in 1989. Acceptable energy transfer and negligible tissue waming data has been published. Our earlier reports involve trials with smal groups of many design iterations. In this study, six transformers of a fixed design, were buil and implanted in dogs for periods from 18 to 28 days. A cylindrical $(26 \mathrm{~mm}$ long by $22 \mathrm{~mm}$ diameter) ferrite-cored primary coil is placed within a small ileal pouch and a torroidal (31.5 mm ID, $9.5 \mathrm{~mm}$. thickness/width) secondary coil surrounds it. Primary wires exit through a miniature stoma or Witzel tunnel: Secondary wires traverse a cervical exit site to apparatus for measurement and dissipation of transmitted power. Al! survived to elective ternination without complications. The full power transfer goal $(15 \pm 2 \mathrm{~W}$ at $10 \mathrm{~V}, 163 \mathrm{KHz})$ was achieved in all and maintained for 7 to 16 days. Coil heat loss at this power level by calorimeter is $1.75 \pm 0.25 \mathrm{~W}$. Tissue warming (thermistor fixed on inner surface of secondary) was 0 to $1.2^{\circ} \mathrm{C}$ over peritoneal reference. Mucosa showed no substantial histologic damage. Despite a design not yet optimized for efficiency, these data support the postulated surgical feasibility and effective heat dissipation of a power transformer, positioned within a small enteric pouch, transmitting a quantity of power useful for circulatory support. The goal of current study is optimization of core material, and of mechanical and electrical design, based on computer inductance modeling. 
99

GEOMETRIC CONSIDERATIONS IN THE DESIGN OF MICROCAPSULES FOR A HYBRID ARTIFICIAL PANCREAS

Michael H. May, Michael V. Sefton

Department of Chemical Engineering and Applied Chemistry, University of Toronto, Toronto, Canada.

A Thiele modulus/effectiveness factor analysis and process control modeling were used to assess microencapsulated islets used as a hybrid artificial pancreas. Comparisons were made on the basis that an ideal antificial pancreas would respond in near physiological time, would consist of a minimum number of islets and would occupy a minimum volume. The first requirement is related to the function of the device, the second to the limited availability of islets and the third to the simple constraint on the volume that can be implanted. Results indicated that a spherical capsule, with an outside diameter only slightly larger than the islet it surrounds, and with a membrane insulin permeability that is an order of magnitude larger than the current estimate for our hydroxyethyl methacrylatemethyl methacrylate (HEMA-MMA) microcapsules would satisfy the basic requirements of an artificial pancreas. For $150 \mu \mathrm{m}$ diameter islets, this corresponds to a capsule approximately $200 \mu \mathrm{m}$ in diameter, with a $25 \mu \mathrm{m}$ thick membrane wall (i.e., a negligible stagnant aqueous layer around the islets) and a membrane insulin permeability of $3.5^{*} 10^{-8} \mathrm{~cm}^{2} / \mathrm{s}$. The effects of geometry, size, membrane thickness, membrane permeability and the number of islets per capsule were also assessed.

100

XENOGENEIC TRANSPLANTATION OF MACROENCATSULATED ISLET TISSUE KE Dionne, OD Hegre, DW Scharp ${ }^{\dagger}$, PE Lacy ${ }^{\dagger}$

CytoTherapeutics Inc; twashington University

Immunoisolation provides a mechanism to test the utility of xenogeneic endocrine tissue transplantation therapy in models of Type I Insulin Dependent Diabetes Mellitus. Encapsulation devices are configured in a variety of geometries including hollow fiber and flat sheet with membranes of various cutoffs. Each device is of a size which can contain large numbers $(>100)$ of islets or insulin producing cells and be handled easily during surgical implantation and removal. The physical nature of the membrane surface coming in contact with recipient tissue is controlled to optimize biocompatibility in a site dependent fashion.

Membrane transport properties and device configuration are modified to achieve a pattern and time course of encapsulated insulin release in vitro that is similar to that of unencapsulated islets. In successful devices, delays do not exceed $5 \mathrm{~min}$ following change in glucose stimulus.

Noninsulin treated, non-immunosuppressed streptozotocin diabetic mice have been reversed of hyperglycemia using xenogeneic rat islets encapsulated in either flat sheet or hollow fiber devices, the latter implanted in either the subcutaneous or intraperitoneal site. Success rates exceed $80 \%$ at elective termination at sixty days. In similar experiments, normoglycemia has been maintained out to $8+$ months. Insulin independence has also been achieved in BB rats and NOD mice.

With consistent reproducible success in large animal models, immunoisolation therapy by macroencapsulation may prove efficacious in the clinical treatment of human diabetes; overcoming the constraint of human islet tissue supply, providing a retrievable (replaceable) therapy for reestablishing normal carbohydrate metabolism without immunosuppression.

101

POLY(ETHYYENE GI YCOL) COATINGS OF ISLETS OF LANGERHANS Cruise, GM, Sawhney, AS, Pathak, CP, Luther, KM, Hubbell, JA

Department of Chemical Engineering, University of Texas, Austin, TX, 78712

The potential of treating certain diseases, such as diabetes, with allogenic or xenogenic tissue transplantation is high. However, the transplanted tissue is often rejected by the host's immune system. Microencapsulation of the transplanted tissue in a biocompatible, permselective membrane shows promise for alleviating the problems of graft rejection. Nutrients, wastes, transport proteins and all other low MW compounds would freely permeate the membrane, but higher MW compounds such as immunoglobulins and complement proteins would be unable to diffuse through the membrane.

We have developed a method of photopolymerizing a thin layer of polyethylene glycol (PEG) directly on the surface of islets to act as a permselective membrane for transplantation. This method has several
advantages over conventional microencapsulation, namely reduced diffusion barriers and transplantation volumes. The islets are exposed to an eosin $Y$ solution and then suspended in a $23 \%$ w/w PEG solution with triethanolamine and vinyl pyrrolidinone and irradiated with an argon laser. The excess PEG solution is washed away, leaving islets with a conformal, 20 micron coating of PEG.

Coated islets show no significant difference from control islets in viability using ethidium bromide and fluorescein diacetate staining. No significant difference was observed between coated and control islets' ability to secrete insulin in repsonse to glucose using a static glucose stimulation procedure. Additionally, the microstructure of coated islets is normal using transmission electron microscopy. Presently, the permeability of conformal coatings with various PEG compositions are being investigated.
102

THE NEOVASCULARIZED BIOARTIEICIAL PANCREAS ORGANOID RL Foumiert JG Sarver*, RJ Mellon+, PJ Goldblatt*, JM Homero SE DeMernitie, TL Pharese, SH Selman's

Dept. of Chemicd Enginearing, Uiviversity of Toledo; Depl. of Puthutugy". Dept. of Fediaticse, Dept. of Urology/Surgerys, Medical College of Ohio, Toledo, Ohio

A neovascularized bioartificial organoid is being investigated as a vehicle for the transplantation of therapeutic cells. For example the islets of Langerhans for the treatment of diabetes. In the organoid approach a fibroblast growh factor is used to facilitate the growth of capillaries into an inert support matrix adjacent to a membrane enclosed compartment that contains the cellular material. This approach provides an environment with a nonclotting blood supply in which the transplanted cells can perform their therapeutic function without the danger of immune rejection. Several techniques are being used to quantify the blood supply and to characterize the mass transfer characteristios of the device. Blood flow rates to the device have been measured using radioactive microspheres. A tracer molecule technique has been developed to measure the mass transfer rate from the cellular compartment of the device into the body fluids. Results will also be presented showing the level of glucose controf achieved using porcine islets in diabetic rats.

103

BLOOD GLUCOSE REGULATION USING A SLIDING SCALE ALGORITHM S. M. Abraham, J .H. Strickland Jr., MD., M. J. MoCutcheon, Ph. D. BME Dept., The University of Alabama at Birmingham, Birmingham, AL 35294

Many of the current algorithms for closed-loop regulation of blood glucose level (BGL) consist of mathematical equations which vary insulin infusion as a function of glucose kinetics. As a result, these equations can become quite complex since they are based on the assumption that the current insulin infusion rate is a combined function of current and prio glucose and insulin levels, infusion rates and time. Also, they may introduce observer bias because of human preconceptions of how gluoose-insulin interactions occur in-vivo. The algorithm developed for this study uses a simple, self-correcting bok-up table to continually adjust the insulin infusion rate in direct proportion to the BGL and concentration change with time. Thus, the algorithm implements a simple proportional-derivative controller based on what physicians call a sliding scale. Observer bias is minimized because the adjustments to the infusion rate are based upon the "experience" which the controller gains from historical glucose level changes. The algorithm was used to predict the insulin infusion rate required to clamp BGL in a set therapeutic range $(125-175 \mathrm{mg} / \mathrm{dl})$ in computer simulations and (15) patient clinical trials on Type I and II diabetics. Current results show the miean clamp level at the end of the 12 bour study specification to be $172 \pm .707 \mathrm{mg} / \mathrm{dl}$, and the mean clamp level during the last 4 bours to be $155 \pm 13.5 \mathrm{mg} / \mathrm{dl}$. Total algorithm code size is $21 \mathrm{~K}$. These results support the hypothesis that a sliding scale scheme can be used to achieve efficient glucose clamping. Applications of the algorithm are a microcomputerbased controller or possibly an implantable artificial pancreas closed loop system. 
105

IN VITRO AND IN VIVO STUDIES ON QUANTITATIVE MAPPING OF TURBULENT STRESSES DOWNSTREAM OF MECHANICAL AORTIC HEART VALVES

H. Nygaard, P. K. Paulsen, J. M. Hasenkam, E. M. Pedersen, P. G. Walker and A. P. Yoganathan

Georgia Institute of Technology, Atlanta, GA and Aarhus University Hospital, Aarhus, Denmark

Comparative in vitro and in vivo studies of velocity fields downstream of different designs of artificial aortic valves have shown similar quantitative results. However, quantitative turbulence measurements in vivo are virtually non-existent, mainly due to the lack of necessary intraoperative techniques and instrumentation for accurate measurements.

The purpose of this study is to compare quantitative in vivo turbulence measurements obtained intraoperatively using a new perivascular ultrasound Doppler technique, with in vitro measurements obtained with a two dimensional laser Doppler anemometer system.

The in vitro and in vivo turbulent stress measurements indicate that the magnitude of the stresses are large enough to cause sub-lethal and/or lethal damage to blood elements, such as red cells and platelets.

106

INLET-OUTLET VALVE HEMODYNAMICS IN THE NOVACOR LEFT VENTRICULAR ASSIST SYSTEM (LVAS)

Harvey S. Borovetz, Richard D. Schaub, Frank D. Shaffer, and John C. Woodard

University of Pittsburgh, Pittsburgh Energy Technology Center, and Novacor Division, Baxter Healthcare Corporation

This work evaluated residence times and flow characteristics in regions local to the inflow and outflow valves of the Novacor LVAS (Novacor Division, Baxter Healthcare Corporation). The influence of valve housing geometry and pump hemodynamics on the flow characteristies in these areas was of specific interest in this study. Residence times were measured under clinically relevant hemodynamic conditions in the inflow valve region of four different valve housing geometries: $21 \mathrm{~mm}$ triple sinus, $21 \mathrm{~mm}$ no sinus, $21 \mathrm{~mm}$ shallow sinus and $25 \mathrm{~mm}$ shallow sinus. Velocities were sampled at six times during the pump cycle corresponding to different phases of pump systole and diastole.

Key results of this study are: i) significant differences ( $p<0.05$ ) in residence times in the inflow valve region were obtained for the various housing geometries tested; ii) both flow rate inflow valve region were obtained for the various housing geometries tested; ii) both flow rate through and motion of the valve leaflets influence the magnitude of velocities measures in the areas of interest; iii) higher velocities were measured adjacent to the outflow (versus inflow)
valve; iv) the magnitudes of the measused velocities varied as a function of time during the pump cycle. These data suggest that global hemodynamic factors such as pump output and frequency strongly influence the local hemodynamirs in the areas of interest. Not unexpectedly the geometry of the inflow valve housing governs, in part, the flow characteristics local to the inflow valve. These findings imply the possibility of improved "valve washing" with modifications to the valve housing design and operation of the pump at increased frequencies and pump outputs.

107

COMPARISON OF CAVITATION FIELDS FOR MONOLEAFLET MECHANICAL HEART VALVES C.S. Lee, K.B. Chandran

Department of Biomedical Engineering, University of lowa, lowa City

Three types of heart valves having mono-leaflet tilting disc as an occluder were investigated to clarify the cause of cavitation bubbles developing at valve closure and to delineate the effect of valve design on the cavitation field. Simultaneous recordings of the pressure waveforms and photographs of the inflow surface of the valve were attempted using the flow chamber simulating the mitral valve closing event under the controlled ventricular pressure rise rate (dp/dt). Vapor bubbles were observed around the inflow metal strut (stopper) of the valves. Pressure waveform measured at the same region showed an abrupt fall below liquid vapor pressure $(-700 \mathrm{mmHg})$ at the beginning of the impact, followed by pressure oscillations about the liquid vapor pressure for less than $0.5 \mathrm{msec}$. MedtronicsHall and Jomed valves, having the stopper at the major orifice area, were found to cavitate far below the physiological loading rate (estimated to be $750 \mathrm{mmHg} / \mathrm{sec}$ ) while Bjork-Shiley valve having the stopper near the central diameter of the orifice did not show cavitation bubbles up to $1500 \mathrm{mmHg} / \mathrm{sec}$. Medtronics-Hall valve with larger stopper showed larger intensity of bubbles compared to Jomed valve. On the other hand, the Jomed valve was found to have lower threshold loading rate for cavitation incipience. The results of the study indicate that cavitation in prosthetic heart valves is caused by the flow field generated by the squeezing of fluid between the stopper and occluder due to the impact of the two at the instant of valve closure. Intensity of cavitation field is found to depend on the location and size of the stopper. Flexibility incorporated in the disc material of the Jomed valve is not found to have a considerable effect on the cavitation incipience.
108

DECELERATION OF BILEAFLET MHV AT CLOSING AND TRANSIENT PAESSURE REDUCTION

S.K.Wang, Z.J.Wu, Y.Wang and N.H.G.Hwang

Department of Biomedical Engineering, University of Miami, Coral Gables, FL 33124 .

Early results from this laboratory have shown that the leaflet impact velocity at closing of a mechanical heart valve (MHV) may hold the key to valve sound and to in vivo cavitation of MHV. The contribution of the transient squeeze flow in the gap space between the closing occluder and the valve housing has been reported elsewhere.

MHV leaflets were found to rapidly decelerate inmediately before impact the valve housing. The drastic deceleration is believed to generate a transient pressure reduction (Ptr, or commonly termed as "water hammer pressure") on the in-flow side surface of the MHV occluder. Based on the measurements using the laser sweeping technique (LST), the final stage MHV leaflet closing decelerations (LCD) were calculated for $29 \mathrm{~mm}$ St Jude Medical (SJM) mitral MHV and a new equivalent size 1205 bileaflet MHV. The LCD measurements were found to vary from beat to beat, but were distributed within a finite range. The mean values of $L C D$ increase with the heart beat rates for each MHV tested. The leaflet closing deceleration at 120 beats/min of the $1205 \mathrm{MHV}$ was measured to reach $-5000 \mathrm{~m} / \mathrm{sec}^{2}$, while that for the SJM was $-14000 \mathrm{~m} / \mathrm{sec}^{2}$. Assuming a stroke volume (SV) of $66 \mathrm{~cm}^{3}$ at $120 \mathrm{bpm}$, and a dynamic closure volume of $5 \%$ SV for both valves, the estimated magnitudes of Ptr for the two MHVs were -25 $\mathrm{KPa}$ (or $-190 \mathrm{mmHg}$ ) and $-70 \mathrm{KPa}$ (or $-530 \mathrm{mmHg}$ ) respectively.

109

IMPACT AND REBOUND OF MECHANICAL HEART VALVE(MHV) LEAFLETS AT CLOSING

Z.J. Wu, S, Menon and N.H.C Hwang

Department of Biomedical Engineering, University of Miami, Coral Gables, FL33124

The terminal closing behavior of MHV leaflets was monitored using a laser sweeping techniqug(LST) which measures the angular displacement of the MHV leaflet with microsecond precisions. The test valve was installed in the mitral position of a physiological mock circulatory loop which mimic the geometries of human left ventricle and aortic root. The system includes a computer-controlled preload driven by a textbook left ventricular volume curve, which allows variations of stroke rate, stroke volume and systolic/diastolic ratios; and an adjustable afterload to generate physiological ventricular and aortic pressure waveforms.

This communication elucidates the optical arrangements and technical considerations involved in the application of the LST technique for measuring the leaflet closing velocity in the mock circulatory loop. The measured results and the characteristics of the leaflet movements at closing in two types of commercially available MHVs are presented. The impact and rebound of the leaflets in a bileaflet St. Jude Medical $29 \mathrm{~mm}$ mitral valve and a monoleaflet Medtronic Hall $29 \mathrm{~mm}$ mitral valve are compared.

110

EFFICACY OF THE GORLIN FORMULA FOR THE ASSESSMENT OF PROSTHETIC VALVE FUNCTION

Flavio B. Souza-Campos and Richard T. Schoephoerster
Mechanical Engineering Department, Florida International University, Miami, FL 33199

Natural and prosthetic valve function is commonly assessed in cardiac catheterization laboratories by effective valve area estimates using the Gorlin equation and measurements of mean valve flow and pressure drop. However, large errors have been found using the Gorlin formula for the prediction of effective opening area of prosthetic valves. The Gorlin formula is derived from hydraulic equations of conservation of mass and energy and contains a constant empirical discharge coefficient to adjust the equation for energy losses in the wake region of the valve. We have previously shown that this discharge coefficient varies with Reynolds number as well as the geometry of the valve itself. In this study, we test the efficacy of the Gorlin formula for the prediction of effective orifice area of several designs of valve prostheses (bileaflet, tilting disc, and tissue) in states ranging from normal to mildly to severely stenotic. The valves were mounted in the mitral position of a flexible, in vitro model of the left ventricle. The pumping action is produced by a piston driven by a step motor where the velocity of the piston is controlled with a microprocessor to produce physiologicat mitral and velocity of the piston is controlled with a microprocessor to produce physiological mitral and aortic flow. The left ventricular model is incorporated in a mock flow loop containing characteristic and peripheral compliance and resistance to produce physiological pressure wavefoms. The system was run al heart rates ranging from 50 to 140 beatshin and cardiac outpuls of 3 thersimin and measurenenss of miral valve flow, pressure drop, and actual projected orifice areas were preliminary results indicate a strong dependence of discharge coeficient with valve flow rate. geometry, and degree of stenosis. Based on these preliminary results, the efficacy of the
Gorlin formula for the evaluation of prosthetic valve function is quite suspect at best. 
111

HEMODYNAMIC BEHAVIOR OF VENOUS VALVE: IN VITRO QUANTIFICATION BY LASER OPTICS

Y.C.Qiu, S.K.Wang, N.H.C.Hwang

Department of Biomedical Engineering, University of Miami, Coral Gables, FL 33124.

The dynamic performance of the glutaraldehyde treated bovine jugular vein valves (VV) was studied using both stented and unstented models. The experiment was carried out in a hydraulic system which provides a steady energy source for desired discharge rates. An adjustable oscillatory pulse was superimposed to the downstream of the testing valve to simulate the intrathoracic pressure changes. A $10 \mathrm{mw} \mathrm{He}-\mathrm{Ne}$ laser beam was expanded and projected along the axial direction to cover the entine valve surface area with uniform intensity. After passing through the opened valve area, the laser ligbt was collected by a photodetector to allow quantification of the valve opening area. A second 5 mw He-Ne laser beam was optically dispersed by a cylindrical lens to form a parallel light stee: that orthogonally intersects the extercal diameter of the compliant valve conduit to allow continuous monitoring the diameter change. A cannula type electromagnetic flow probe was placed upstream of the testing valve to measure VV flow rate. Using an aqua-glycerol solution ( $33 \%$ by volume), the experiment was carried out to quantify the relationships between the steady pressure drop, discharge rate, and VV opening area. The experimental Reynolds number (Re) was defined by the equivalent circle diameter of the opening area. For the stented VV, the cusps were found extremely sensitive to the flow rate that the opening area reaches $95 \%$ of total opening at $R e=397$. The pressure-drop coefficient, $C_{i}$, reaches peak value of 5.8 at $\operatorname{Re}=88$, and gradually decreases to a near constant 0.3 when $\operatorname{Re} \geq 1050$. For the unstented VV, diameter dilation was monitored together with the pressure and flow. The soft VV conduit diameter dilates at closure and at sudden opening. The outflow field was observed to change with the oscillatory pressure gradient. LDA measurements indicale no reversal motion in the central core.

\section{2}

FINITE ELEMENT MODELING OF THE AORTIC ROOT AND VAI.VE KJ Grande, KS Kunzelman, RP Cochran, TL Richards, "TE David, ED Verrier Bioengineering, Cardiothoracic Surgery, \& Radiology, $U$ of Washinglon, "U of Toronto

We are using magnetic resonance imaging (MRI) to develop a morphologically exact 3-D FEM of the aortic root/valve system for evaluation of nomal and pathological valve function. Previously, finite element modeling (FEM) has only been applied to the valve (native or prosthetic) and assumed geometric shapes have been used. Moreover, no mechanical FEM's have included the aortic root in their analyses.

MRI was performed to obtain axial serial sections of the ex vivo calf aortic root and valve. The resulting MR images were analyzed digtaliy to oblain cross-sectional outlines of the root and valve (pressurized and fixed in position by infusing silicone nubber). Each outline was processed further to obtain 3-D coordinate points, which will be used to recreate the aortic root shape for finite element optimization of root replacement designs. Finite element analysis solves the matrix oquation $E=K D$ where $E$ is the force vector, $D$ is the displacement vector, and $K$ is the stiffness matrix. Following solution, the entire stress profile of a discretized object may be examined. For our model, we will input the nondeformed geometry of the aontic root, physiological For our model, we will input the nondeformed geometry of the aortic root, physiologica
pressures, and the material properties of the aortic root and valve. Model results will be compared with MR images of physiologically pressurized aortic roots. Surgical repair and replacement techniques, which are integrally related to the aortic root dynamics, will also be examined within the FEM.

An additional goal of our investigation is to determine the optimal size, conformation, and material type for the development of a new aortic root vascular gratt. This graft is being designed for use in a surgical technique which would leave the native aortic valve intact. Ideally, the graft will perform the same function as the aortic sinuses in contributing to aortic valve closure.

\section{3}

COMPUTATIONAL ANALYSIS OF AORTIC VALVE DYNAMICS IN NATURAL AND PROSTHETIC AORTIC ROOTS

Vinod B. Makhijani ${ }^{1}$, H. Q. Yang ${ }^{1}$ and Mano J. Thubrikar ${ }^{2}$

('CFD Research Corporation, Hunisvilje, AL 35805. ${ }^{2}$ Heineman Medical Research Center, Charlote, NC 28203)

We study aortic valve performance in the natural aortic root (sinus diameter $=1.64 \mathrm{x}$ valve annulus diameter, Do) at different left ventricular dp/dt by using a computational, twodimensional, fluid-structure interaction model for aortic valve and blood flow dynamics. We also use the model to study valve dynamics in the absence of the sinuses in aortic root prostheses by simulating valve motion in two, tubular, aortic root grafts (1.12 Do and 1.25 Do diameter). Physiologic ventricular (peak pressure $115 \mathrm{~mm} \mathrm{Hg}$ ) and aortic $(115 / 75 \mathrm{~mm}$ $\mathrm{Hg}$ ) pressure boundary conditions ( 75 beats/min) are used in the calculations. Fully coupled, time-accurate, cycle-independent solutions for leaflet configuration and flow field are obtained at $2 \mathrm{msec}$. intervals during an entire flow cycle. In the natural aortic root, when the average ventricular dp/di dusing valve opening is decreased from 1432 to $907 \mathrm{~mm}$ $\mathrm{Hg} / \mathrm{s}$, valve opening time increases from 22 to $34 \mathrm{msec}$, and peak aortic flow velocity decreases from 80.5 to $42 \mathrm{~cm} / \mathrm{sec}$. In the cubular graft simulations, we observe severe flutter near the fully open leaflet tip in the smaller graft. Also, regurgitation is higher $(6.15 \%)$ in this case as compared with the natural aortic root $(2.02 \%)$. In the larger graft, leafle flutter is absent. Also, vortex formation in the smaller region above the leaflet results in slightly early valve closure (by $4 \mathrm{msec}$ ) as compared with the natural aortic root. Hence, regurgitation is lower $(1.66 \%$ ). In both grafts, the peak fluid shear stresses above and below the valve surface are significantly higher than in the presence of the sinuses. These preliminary results indicate that: a) valve function in the natural aortic root could be adversely affected by the weakened contractility of the cardiac muscles; b) absence of the sinuses in tubular grafts could compromise aortic valve performance.
114

EFFICACY OF THE NEW ST-JUDE MEDICAL SMALL SIZE AORTIC VALVE O.Salat and N.H.C.Hwang

Department of Biomedical Engineering, University of Miami, Coral Gables, FL 33124

The hydraulic performance of a snall size (19mm) St.Jude Medical (SJM) aortic heart valve prosthesis was investigated in an experimental mock circulatory flow loop using aquaglycerine solution ( $33 \%$ by volume) as the blood analog solution. The new bileaflet valve (HP stands for Hemodynamic Plus) has the suture ring redesigned in such a way that the orifice diameter is increased by $2 \mathrm{~mm}$. This allows a $25 \%$ larger flow area as compared to the traditional SJM model. The $19 \mathrm{~mm}$ HP model was tesled for a heart rate of 70 beats $/ \mathrm{min}$ and for different flow rates (ranged from 2.5 to 4 liters $/ \mathrm{min}$ ) at constant pressure levels. The traditional SJM 19 valve was used as the study control.

The first results indicated that the mear pressure drop foberined by integrating the differential pressure between the left ventricle and the aorta) in the HP modlel reduced by a measurable amount as compared to the traditional SJM model, under the same flow rates and pressure levels for all three pulse rates $(70,90$ and 120 beats per minute) tested. These results were in accordance with the mean pressure drops measured in clinical implants made on the traditional model.

115

A LONGER BODY MECHANICAL HEART VALVE FOR IMPROVED HYDRODYNAMIC PEAFORMANCE.

S.Menon, W.Li, Z.J.Wu, A.Haubold, N.H.C.Hwang

Department of Biomedical Engineering, University of Miami, Coral Gables, FI 33124 "Carbon Implants, Inc., Austin, Tx 78754

Investigations of the hemodynamic performance of the new 1205 bileaflet mechanical heart valve (BMV) have shown that the profile of the valve has a signiticant effect on its hydrodynamic performance. Conventional BMV manufacturers prefer 'low profilg' valve bodies, although thers has been no documented evidence that suggest such a configuration enhances the hemodynamic performance. The 1205 BMV has a larger annular valve body formed with an interior sidewall which defines central passageway in the downstream direction. The valve leaflets are mounted within the valve body and open to a maximum angle of $90^{\circ}$, to provide a low resistance to forward flow. The leaflets close at $45^{\circ}$; which manifest a significantly smaller excursion compared to the current BMV.

The energy loss through a MHV is a result of frictional and form losses through the valve orifice, however the latter often dominates. The geometry of the new 1205 valve body offers a significantly lower form loss than conventional MHV designs thus reducing the overall energy loss of the valve. The longer valve body provides the 1205 with improved operating characteristics as indicated by the resuits of studies on leaflet closure velocity. The studies have demonstrated that the 1205 ENV has a significantly lawer terminal closing velocity than the well established St. Jude MHV in the ranges studied. These results suggest that the new 1205 BMV provides superior hydrodynamic characteristics than currently available MHV while also reducing the potential for inception of cavitation.

\section{6}

AN $I N$ VITRO INVESTIGATION ON THE EFFECTS OF OFF-AXIS POSITIONING OF HANCOCK I AORTIC BIOPROSTHESES

Jeffrey M. Gross

Heart Valve Division, Medronic Inc., Irvine, Caljomia

An in vitro study was conducted to determine possible structural, wear, and hemodynamic effects of implanting a bioprosthetic valve at an angle relative to the flow field in the aortic position. The non-coronary leaflet of four $25 \mathrm{~mm}$ Hancock I Aortic valves were mounted super annularly at $0^{\circ}, 3.3^{\circ}, 6.7^{\circ}$, and $10^{\circ}$. Each valve's hemodynamic performance was evaluated using a physiologic pulse duplicator and steady flow system prior to fatigue testing (1450 Beats/min) and at every subsequent 40 million cycles. Visual inspections were conducted initially and every 40 million cycles to document structural and wear characteristics of the valves.

No statistically significant differences exjsted with regard to hemodynamic performance for any valve over time $\left(p<0.05\right.$ ). Wear on the $3.3^{\circ}$ and $6.7^{\circ}$ mounted valves exhibited normal patlerns as did the control valve. The $10^{\circ}$ valve showed significant accelesation in wear patterns beginning at 120 million cycles, i.e. abrasions and boles resulting from tissue contact with the bias strip and tears in the free margin. In addition, the non-coronary leaflet was flattened at the tunica, and the free margin curled over that of the other two leaflets.

Based on this initial study, it hypothesized that the permanent deformation of the noncoronary leaflet and excessive wear patterns experienced by the $10^{\circ}$ valve may be due to the altered force structure seen by the non-coronary leaflet as a result of the off-axis mounting. It is important to note that the wear characteristics as a function of the off-axis position for a particular bioprosthetic valve cannot be generalized to all bioprostheses and must be experimentally determined for each valve design. Future studies will seek to validate this hypothesis for different valve designs in statistically significant numbers. 
117

FLOW DYNAMICS IN FONTAN CONNECTIONS: PRELIMINARY IN VITRO STUDIES

Young H. Kim, Peter G. Walker, Amold Fontaine and Ajit P. Yoganathan Cardiovascular Fiuid Mechanics Laboratory, School of Chemical Engineering, Georgia Institute of Technology, Atlanta, GA 30332-0100, U.S.A.

The Fontan operation is performed for surgically correcting congenital right heart defects such as pulmonary/tricuspid atresia or unj-ventricular defects. Flow visualization and pressure mapping studies with anatomically correct Pyrex models were performed in vitro under steady flow conditions to study the flow dynamics in atrio-pulmonary connections. Since the graft conduit is connected with a curvature between the right atrium and the main pulmonary artery (MPA), swirling flow is the main flow characteristic. Flow separation was limited and the axial flow in the MPA became skewed toward the left wall. With a 50:50 flow-split-ratio between the left and right pulmonary branches, more significant secondary flow was observed in the right pulmonary artery (RPA). The proximal model whose conduit was made between the right atrium and the proximal location of the pulmonary valve has more swirling flow in the MPA. From the pressure mapping studies, it was seen that the major pressure drop occurred at the junction of the conduit and across the branches. The proximal model had large pressure drops compared to the distal model and the The proximal model had large pressure drops compared to the distal model and the
left pulmonary artery (LPA) had larger pressure drops than the RPA. This left pulmonary artery (LPA) had larger pressure drops than the RPA. This
preliminary study suggests that the geometry of the conduit plays an important roie in the flow dynamics of Fontan connections and it should be optimized to minimize pulmonary flow resistance.

\section{8}

PROXIMAL ISOVELOCITY SURFACE AREA CALCULATIONS IN EXTREME ENVIRONMENTS: THE LEANING PISA IN CROSSFLOW Edward G. Cape and Gerard J. Boyle

University of Pittsburgh, Chldren's Hospltal of Pittsburgh, PA

Ventricular septal defocts (VSD) are present in $30 \%$ of all cases of congenital heart disease, decreasing the efficiency of the heart and leading to pulmonary vascular disease been achleved. A simplified approach using proximal isovelocity surface areas (PISA) has been effective in correlating valvular regurgitant flows with catheterization grades. Although PISA's are readily obtained in pls with VSD's, this simplified calc itation has no been successful since the PISA is interposed into left ventricular outfliow (LVO), causing a skewed isovelocity surface and misapplication of the mass balance between the PISA and lesion. In vitro modeling and clinical studies were used to address the hypothesis that the PISA calculation can be modified for PISA/crossflow interaction, allowing noninvasive assessment of VSO's. In an in vitro model designed to isolate the interaction between converging VSO llow and LVO, the basic PISA vs $Q_{\mathrm{p}} Q_{\text {, correlation fell into two }}$ groups. Under resting conditions, PISA gverestimated $Q_{p}-Q,(10-110 \%, r=0.96)$. In high outpur states, PISA cosely estimated actual flows $\gamma=0.92 X+9.78, r=.95)$. In clinical studies, $O_{p}-O$, was determined by catheterization in 12 pts (age 1 month -4 yrs) with Isolated perimembranous VSD's. Peak PISA flow correlated with but overestimated $Q_{p}-O_{\text {s }}$ $(\gamma=5.77 X+0.667, r=.88)$. Correcting for the time velocity integral of $V S D$ velocity improved correlation $(\gamma=4.135 X-0.36, r=0.90)$ but still overestimated. Based on the in vitro studies, a dimensionless correction factor was applied and markedly improved agreement $(p<0.01$ ), indicating prospective clinical studies. Conclusions: These studies explain overestimations using the basic PISA calculation and give insight to potential correction factors which may be incorporated into routine echo exams.

COMPUTATIONAL VALIDATION OF THE PROXIMAL ISOVELOCITY SURFACE AREA METHOD FOR ESTIMATTNG MTTRAL, REGURGITATION IN THE PRESENCE OF VENTRICULAR OUTFLOW

Joanne Hopmeyer, Young H. Kim, Ehud Schwammenthal, Robert A. Levine, Ajit P. Yoganathan.

Georgia Instiate of Technology, Atlanta, GA and Massachusetts General Hospital, Boston, MA

Recent studies have shown that the proximal isovelocity surface area (PISA) method can quantify valvular regurgitation. This method assumes that the fluid converges radially and uniformly cowards an orifice creating concentric isovelocity layers. Using color Doppler flow mapping, and adjusting the Nyquist limit, the velocity will alias as the fluid accelerates towards the orifice. From the color flow map, the radius ( $R$ ) can be measured as the distance where the velocity aliases. The purpose of this study was to determine whether this method could be applied accurately in the presence of ventricular outflow, which can distort the PISA field. Finite difference solution of the Navier-Stokes equations was therefore performed using Fluent in an anatomically correct 3-dimensional ventricular model. Flow was driven through 4-6 $\mathrm{mm}$ diameter regurgitant orifices by a $100 \mathrm{mmHg}$ pressure gradient. Each orifice size was located centrally, or near the outflow tract or posterior wall. Regurgitant flow rates $(Q)$ ranged from 41 to $101 \mathrm{ml} / \mathrm{sec}$. For each aliasing velocity $(V)$ and PISA radius $(R)$ along the centerline of the orifice, the flow rate was calculated by $Q c=2 \pi^{2} V$, To account for underestimation near the orifice and overestimation far from it (finite chamber and outflow effects), a polynomial was finted to plots of $Q c$ versus $R$, and $Q c$ at the point of inflection was taken as the best estimate of actual $Q$. The results suggests that $Q c$ at the point of inflection agrees well with actual values (mean error $=4.3$ $\mathrm{ml} / \mathrm{sec}$, or $6.1 \%$ ), successfully accounting for potential overestimation, particularly nearest the outflow. This computational study confirms that ventricular outflow can distort adjacent PISAs, but that flow rate can still be calculated accurately by analysis of the proximal flow field in a way that could potentially be automated.
120

NUMERICAL MODEL FOR BLOOD FLOW AND AORTIC VALVE DYNAMICS. Vínod B. Makhijani ${ }^{1}, H$. Q. Yang ${ }^{1}$ and Mano J. Thubrikar ${ }^{2}$

$\left({ }^{1} \mathrm{CFD}\right.$ Research Corporation, Huntsville, AL 35805. ${ }^{2}$ Heineman Medical Research Center, Chariotte, NC 28203)

A numerical, two-dimensional, planar, fluid-structure interaction model is developed for studying aortic valve and blood flow dynamics. The computational domain comprises of the ventricular outfow tract, sinuses (diameter $=1.64 \times$ valve annulus djameter) and part of the ascending aorta (one diameter length). The domain walls are assumed rigid. The leaflet is modeled as a thin, non-linear elastic beam. A radial strip of the leaflet from base to nodule of Arantius is taken as the representative section. Uniaxial stress-strain curves are used to describe its structural properties. Blood flow is modeled as laminar, incompressible, Newtorian flow. Leaflet configurations are obtained from the variation in bending moment along their length due to the fluid-imposed load. The unstressed leaflet configuration is assumed to lie between the diastolic and systolic positions. The finite volume, computational fluid dynamics code, CFD-ACE, developed at CFD Research Corporation, is used to solve the Navier-Stokes equations. Structure dynamics and fluidstructure interactions are embedded within the iterative procedure of the code. A.60 x 34 computational grid is used. The leaflet length is sub-divided into 16 elements. Physiologic ventricular (115 $\mathrm{mm} \mathrm{Hg}$ peak pressure) and aortic $(115 / 75 \mathrm{~mm} \mathrm{Hg})$ pressure waveforms (75 beats/min) are imposed at the boundaries. Fully coupled, cycle-independent, time accurate solutions of the flow field and leaflet deformation are obtained at intervals of $2 \mathrm{msec}$. over an entire cardiac cycle. The model predicts the formation of vortices in the aoric sinuses. Model predictions also show fairly good agreement with published in vivo data on: 1) radial leaflet configurations, 2) leaflet displacement, 3) valve rapid opening ( $22 \mathrm{msec}$ ) and closing $(20 \mathrm{msec})$ times, 4) peak aortic flow velocity $(80.5 \mathrm{~cm} / \mathrm{s})$, and 5) regurgitant flow fraction $(2.02 \%)$. The model can be used to study valve performance under various flow conditions.

121

THE SQUEEZE FLOW FIELD OF A MECHANICAL HEART VALVE AT CLOSING

$$
\begin{gathered}
\text { D. Bluestein and N.H.C. Hwang } \\
\text { Department of Biomedical Engineering } \\
\text { University of Miami, Coral Gables, FL } 33124
\end{gathered}
$$

In vivo structure damage to a few clinical Edwards Duromedics mechanical heart valves (ED-MHV) have been reported recently. Electron micrographs of the damaged Pyrolytic carbon (PC) explants indicated surface morphology similar to that of cavitation erosion. Upon examining the parameters which may lead to in vivo cavitation inception, it was established that the closing velocity of the leaflet is the key to MHV cavitation potential. During the final phase of valve closing, the fluid mass in the gap space between the closing leaflet and the valve body is squeezed into motion by the approaching boundaries. The flow pattern created by this motion (termed "squeeze flow"), is related to the geometry of the approaching boundaries, and the terminal velocity of the closing leaflet. The closing bebaviour of the $29 \mathrm{~mm}$ ED-MHV "old spec" was studied by mounting the valve in the mitral position of a physiologic mock circulatory test chamber. The leaflet motion was monitored by a laser sweeping techniques (LST) with precision of 5 microsec interval. The leaflet velocity and the valve boundary geometry were used as the input to determine the squeeze flow field in the gap space. Preliminary computations were made using the FIDAP software package. With $₫$ constant leaflet closure velocity of $4 \mathrm{~m} / \mathrm{s}$ assumed, the two dimensional, time dependent model flow field show that transient flow velocity in the gap space can reach values as high as $30 \mathrm{~m} / \mathrm{s}$ in regions near the edge of the inflow surface of the ED-MHV leaflet. This high speed stream ejected from the gap channel can create the conditions that characterize cavitation. The location of the isolated high speed region can be correlated to the surface erosion that was observed in number of damaged ED-MHV explants.

\section{2}

ROLE OF VALVE DYNAMICS IN BLOOD DAMAGE

T.M. Lamson, L.A Garrison, J.A. Frangos, S. Deutsch", D.B. Geselowitz and J.M. Tarbell

Bioengineering Program and *Applied Research Laboratory

The Pennsylvania State University, University Park, PA 16802

Hemolysis and blood cell activation in flow through prosthetic heart valves are associated with three phases of the valve flow cycle: forward flow through the open valve, regurgitant back flow through the closed valve, and valve closure. The relative contributions of these phases to the overall hemolysis rate of mechanical disk valves will depend on the valve closure dynamics which affect cavitation and possibly other mechanisms of cell damage associated with valve closure.

We have studied single leaflet tilting disk valves in several novel in vitro flow loops to estimate the contributions of the three phases of valve flow to the hemolysis of fresh porcine blood. We have used high fidelity pressure measurements near the inlet of a mitral valve to determine the intensity of cavitation in blood under various operating conditions which alter valve closure dynamics.

The results indicate that regurgitant back flow can make a contribution to hemolysis which is comparable to that of forward flow even though athe regurgitant flow rate is much lower than the forward flow rate. Cavitation can cause significant hemolysis which correlates with certain characteristics of the power spectrum of the pressure signal. 
123

\section{BIOMEDICAL ENGINEERING'S ROLE IN THERMAL BALANCE \\ Hugh N. Tucker, Ph.D.}

Clintec International, Inc; Deerfield, IL 60010

The first law of thermodynamics states that energy can neither be created nor destroyed. Various forms of energy (thermal, chemical, electrical, mechanical) are interconvertible, but the total energy of any closed system is immutable. Man is not exempt. From a thermodynamic standpoint, the body does not expend energy, but merely converts it from one form to another. The final form into which energy is converted is heat, which cannot be used for work in a biological system and ultimately leaves the body to return to the environment. It has become evident that under nourishment, for even short periods, has an effect on the central nervous system's ability to respond to internal and external stimuli. Thermal regulation is no exception. Critical care medicine is now expanding the concepts of fuel utilization and fuel mix to include heat production. The overall concept of the body as a continuous energy exchange device may be represented as a "black box" with the entry of energy as food and its exit as heat. The associated gas exchange represents two matched balances that must proceed simultaneously - substrate balance that determines body composition and thermal balance that determines body heat content. The exchange of heat between the human body and its environment is accomplished at a rate and direction to preserve an almost constant internal body temperature. Given the critical importance of thermal regulation to the survival of organisms, it is surprising that the knowledge of mechanisms for controlling thermal balance is just beginning to emerge. Studies of calorimetry and indirect calorimetry have focused on the internal combustion of protein, carbohydrate, and lipid fuels with measurement of the resulting carbon dioxide and water. In the future attention will be directed to mechanisms and control of the entire fuel energy heat cycle. Thermal monitoring capabilities combined with multi-compartmental computer modeling will be required to begin to describe the complexities of thermal homeostasis.

\section{4}

NUTRITIONAL THERAPY IN THE HIV INFECTED PATIENT

Terry Yamauchi, M.D.; University of Arkansas for Medical Sciences and Arkansas Children's Hospital

Nutritional variations have been tried in the treatment of the patient infected with human immunodeficiency virus (HIV). Nutritional supplements including vitamins and trace metals bave also been explored. While no single therapy has been universally successful, the improvement in quality of life for these individuals is documented.

125

ETHICAL, LEGAI, AND NUTRITION CONSIDERATIONS FOR FEEDING THE TERMINALLY ILL PATIENT.

Carol O. Mitchell, Ph.D, R.D., LDN.

Department of Consumer Science and Education, Memphis State University, Memphis Tn.

Modern technology, including parenteral and enteral nutrition has had a great impact on the delivery of health care. Patients are surviving catastrophic illnesses that were previously fatal. This has led to much discussion concerning the ethical and legal issues and responsibilities of health care professionals in initiating and withdrawing of nutritional support. As our technology becomes more advanced, this dilemma will become even more complex. This presentation will address some of the ethical, legal, and nutrition issues involved in feeding the terminally ill patient. The primary consideration in any decision must be the wishes of the competent, informed patient or his/her surrogate decision maker, and each situation must be resolved individually. However, guidelines for deciding appropriateness of providing or withholding nutrition support have been developed by several professional organization, and these will be reviewed.
126

DETERMINING EFFICACY OF ELECTRONIC MONTTORING DEVICES.

E. A. Tolley, G.W. Somes, E.S. Bryant and S J. Phelps.

University of Tennessee, Memphis, TN 38163.

Electronic monitoring has become a widely used tool for clinical and diagnostic purposes. Two types of monitoring technologies are continuous recorders and real time systems. Frequently automatic warning systems, primarily visual and auditory alarms, are installed on medical equipment. Consequently, many technologies used to detect physiological abnormalities are programmed by imposing an arbitrarily chosen cutoff point on a continuous scale. Thus, continuous monitoring involves recording of sequential test results over varying lengths of time for each monitored individual. Choice of cutoff to discriminate between normal and abnormal states may alter the efficacy of the device. Furthermore, during monitoring the true disease state of the individual may change between tests. We point out the connection between sequential testing by a electronic monitoring device and repeated screening tests by laboratory-based clinical chemistry. We suggest a practical and flexible method for determining the efficacy of monitoring devices based on the Mantel-Haensze estimator of an odds ratio. We point out the benefits of this method for estimating diagnostic lest indices. From resutting sensitivities and specificities, we produce receiver operating characteristic (ROC) curves to compare performance of two or more monitoring devices.

127

CLOSED-LOOP TESTING OF A CONTROLLED ENVIRONMENTAL LIFE SUPPORT SYSTEM (CELSS) FOR A SPACE-BASED PLANT CULTIVAR

Taylor, H.W. and G. Zrilic

New Mexico Highlands University School of Science and Engineering

We have designed and built a $1.0 \mathrm{~m}^{3}$ cabin enclosure for a Phototron ${ }^{\text {Th }}$ plant growth chamber donated by Pyraponic Industries. The chamber is monitored by an array of temperature and air velocity probes and the cabin enclosure is monitored with $\mathrm{CO}_{2}$. $\mathrm{O}_{2}$, relative humidity and pressure sensors. A condensing heat exchanger provides temperature, ventilation and relative humidity control supervised by a Motorolla $68 \mathrm{HC} 11$ microprocessor. A Hewlett Packard 9000 series 382 instrument controller runs the UNIX software responsible for data collection and archiving as well as highlevel control functions such as photoperiod regulation, atmospheric composition and nutrient delivery. The automated nutrient delivery system provides a constant pressure head in the solid plant growth media as well as an hour each day of soil aeration.

A large number of engineering and life science undergraduate students have been supported in this research by the NASA Joint Venture and NASA Space Technology Development and Utilization Programs and by the NSF Coalition to Increase Minority Degrees. Closed-loop tests have focused on the response of chili cultivars to variations in atmospheric $\mathrm{CO}_{2}$ in order to determine the feasibility of space-based applications. Novel control schemes explored include fuzzy processing of over-sampled delta-sigma data streams.

128

HAZARD ANALYSIS OF THE ESCAPE FROM THE POST-PARTUM SUPPRESSION OF MENSTRUAL CYCLES USING TIME-SERIES NURSING AND ETHNOGRAPHIC DATA Taylor, H.W. and M. Vazquez-Geffroy New Mexico Highlands University School of Science and Engineering

Full or nearly full breastfeeding on demand has proven to be the key factor in the balanced maintenance in human population ecology for more than 100,000 years. Even today, breastfeeding provides significantly more women-years protection from pregnancy than all other natural and artificial methods of regulating fertility combined. With support from the NIH Minority Biomedical Research Support program, we have begun a four-year study of postpartum amenorrhea among the women of rural northeastern New Mexico. The study combines computer cataloging of ethnographic data and a novel approach to discrete event recording using programmable scientific calculators.

A pilot study of highly motivated mothers aware of the fertility-suppressing effect of breastfeeding showed a significant effect of the pattern of infant suckling on the timing of the first cvulation after childbirth. The present study seeks to extend the range of infant feeding behavior in order to assess the potential contraceptive effect of lactation in a geographically isolated population within the United States. Time-series data are used within a Cox proportional hazards model. Time-varying covariates are employed to identify a range of behaviors associated with the delay of postpartum ovarian activity and menstruation. 
129

MINORITY CENTER OF EXCELLENCE PROGRAM: UT MEMPHIS COLLEGE OF PHARMACY.-A NATIONAL MODEL

Eoff, J., University of Tennessee, Memphis, TN 38163

The UT Memphis College of Pharmacy Center of Excellence (COE) is funded in part by the U.S. Bureau of Health Professions Division of Disadvantaged Assistance of the Health Resources and Services Administration. The overall aim of the COE is strengthening the capacity of the College of Pharmacy to train minority students in the health professions. The grant will fund programs to improve minority student recruitment; improve minority student performance and retention; promote faculty/student research regarding minority issues; promote the recruitment, training and retention of minority faculty, and improve information resources and curriculum regarding minority health issues. The UT College of Pharnacy will stage a series of recruitment, instructional, enrichment and research activities over a three year period to accomplish the stated objectives.
131

THE EFFECT OF REGULATORY AFFAIRS ON BIOMEDICAL ENGINEERING DEVELOPMFANTS

DE Ott, MD

Mercer University, School of Engineering

The development of medical devices is a regulated activity. It is international in scope. The type and direction of research and development in biomedical related technologies is influenced by regulatory affairs. This influence of regulation is laced with the guige of credibility under the mantle of public safety. its goal, influenced by a civil service interested in propitiating their positions, is tantamount to asking the fox to guard the chicken coup. The Safe Medical Devices Act (SMDA) of 1990 and the revisions of the International Organization for Standardization (ISO) 9000 series make good medical practice (GMA standards more universal but more cumbersome in application. These will lead to total quality management for device development and production. More science and less politics needs to be the outcome of any Center for Devices and Radiological Health ( CDRH) evaluation and recommendation. Without knowledge of the tangled web of regulatory affairs, biomedical engineering developments are doomed to failure or regulatory procedural prolongation creating paralysis and compromise in research, development and application.
130

ASSESSMENT OF MENTORING AND TEACHER INDUCTION MODELS E. Dean Butler

Director of Research, Center for Research in Educational

Policy, College of Education, Memphis State University

The presentation will focus on two models of beginning teacher induction and mentoring implemented in post-baccalaureate teacher education programs at Memphis State University. Specific features of the induction models used in year-long teaching internship will be described and evaluative results summarized.

Institutional and personal factors that contributed to successful mentoring of beginning teacher interns will be identified. Influential personal factors included mentor status within the school, willingness to forge emotional bonds with the interns, gender and race similarities, previous experiences in mentoring, years of experiences in the classroom, and willingness of mentor to allow interns to attain authority and respect.

Institutional factors included vaxiations in work

assignments, locations of teaching stations within the schools, and role definitions. 
132

MAGROMOLECULAR TRANSPORT ACROSS ENDOTHELIUM AND INTERNAL ELASTIC LAMINA OF NORMAL AND INJURED RAT AORTA

M. S. Penn, S. Rangaswamy, G. M. Saidel and G. M. Chisolm

Dept. of Cell Biol., Cleveland Clinic Fndn.; Dept. of Biomed. Engr., Case Western Reserve Univ., Cleveland, $\mathrm{OH}$

Endothelial cell (EC) injury accompanying atherosclerosis and angioplasty may influence lipid accumulation and transport of growth factors and cytokines. We have studied the permeabilities of the EC layer and the internal elastic lamina (IEL) in vivo in rat aorta in normalcy and after injury by lipopolysaccharide (LPS) hypertension and oxidized LDL (OLDL), in an attempt to understand macromolecular accumulation in the intima and media. We used the enzyme activity of horseradish peroxidase (HRP) as a macromolecular probe because of its intermediate size (44kD) between some growth factors (PDGF, 33kD) and albumin $(69 \mathrm{kD})$. We quantified intimal and medial HRP concentration profiles 15 minutes after IV injection of HRP $(50 \mathrm{mg} / \mathrm{kg})$. Using a novel model of arterial wall transport that defined distinct permeability coefficients for endothelium and IEL, we found the IEL to be responsible for more than $45 \%$ of the barrier function of the normal blood-tissue interface. Using LPS-induced EC injury, we observed that increased HRP transport preceded EC death and proliferative response. LPS caused a transient apparent increase in IEL permeability at $24 \mathrm{hr}$, but a monotonic increase in EC permeability over a $120 \mathrm{hr}$ period oLDL infusion and chronic hypertension both increased EC permeability to HRP. However, chronic hypertension did not alter IEL permeability to HRP. These data suggest the importance of the balance between EC and IEL permeabilities in regulating intimal accumulation and they suggest that the IEL may play a significant role in controlling paracrine communication between intimal and medial cells.

133

MONOCYTE DISTRIBUTION ON THE AORTIC SURFACE IN RELATION TO FOCAL HIGH PERMEABILITY SITES

Susan M. Lessner and Clark K. Colton

MIT, Department of Chemical Engineering, Cambridge, MA

En face studies of the rabbit aortic endothelium indicate a relationship between focal sites of protein accumulation ("high permeability sites") and adherent monocytes We used biotinylated bovine serum albumin (BSA) as a macromolecular tracer to identify high permeability sites in the aortic wall, and non-specific esterase (NSE) staining to visualize adherent monocyles. The distribution of monocytes on the rabbit aortic wall is spatially very heterogeneous, with the highest densities of adherent cells occurring in the aortic arch and the upper descending thoracic aorta (up to 69 cells/sq $\mathrm{mm}$ on average). The number density of adherent cells is also relatively high along and distal to the flow dividers of ostia. In the aortic arch, adherent monocytes are associated with up to $41 \%$ of all high permeability regions. In selected aortic regions chosen for more detailed analysis, the areas of highest monocyte density correspond topographically to regions where high permeability foci are abundant.

134

CHARACTERIZATION OF ELEVATED LOW DENSITY LIPOPROTEIN PERMEABILITY SITES AT LESION-PRONE AREAS OF THE NORMAL RABBIT AORTA Robert A. Herrmann, Richard A. Malinauskas, and George A. Truskey, Department of Biomedical Engineering, Duke University, Durham, NC 27708-0281

En face autoradiography of the endothelium (Circ Res 1992; 71: 883-897) was used to quantify the distribution, size, and permeability of sites of enhanced endothelial permeability to 125 I-low density lipoprotein (125I-LDL) around the intercostal arteries, celiac artery, and at the iliac bifurcation of normal rabbit aortas. Permeable sites were more frequent distal to the intercostal branch orifices, and lateral and distal to the orifice at the celiac branch. At the intercostal branch orifices, these sites were larger with a lower permeability and higher frequency than away from the branch. The mean regional permeabilities, which were an average of low and high permeability sites, were the same at and away from the intercostal branches. At the celiac flow divider, the sites were more permeable and larger than away from the flow divider. Within $0.25 \mathrm{~mm}$ and $1 \mathrm{~mm}$ of the celiac flow divider, the mean regional permeability was, respectively, eight and two times higher than away from the flow divider. Few sites of elevated permeability were found distal to the aorto-iliac bifurcation and the permeabilities at the medial and lateral walls of the iliac arteries were not different. Mitotic cells accounted for $13 \pm 7 \%$ of all sites of elevated permeability and were not increased at branch sites, suggesting that other mechanisms were responsible for increased LDL permeability in the rabbit. The results indicate that the permeability and frequency of occurrence of sites of elevated permeability around the celiac and intercostal branches is associated with the distribution and severity of early lesions in rabbits fed a hypercholesterolemic diet.
135

ROLES OF HEMODYNAMICS AND ENDOTHELIAL CELL TURNOVER IN THE FOCAL NATURE OF MACROMOLECULAR TRANSPORT Shu Chien, Kenji Sakakibara, and Terue Sakakibara Institute for Biomedical Engineering, Univ. Calif., San Diego, La Jolla, CA., and Hamamatsu Univ., Hamamatsu, Japan.

Rabbit thoracic aorta was subjected to systematic topographic mapping of endothelial cell nuclear morphology (used as an index of local blood flow direction and wall shear stress), mitosis and permeability of Evans Blue labeled albumin (EBA). The endothelial nuclear orientation indicated the presence of straight laminar flows along the longitudinal axis of the thoracic aorta and also spirohelical secondary flows. Endothelial nuclei with high shape indices (more spherical) were located mainly in regions with secondary flows, whereas those with low shape indices were primarily found in regions with straight laminar flow. Almost all mitotic cells were located within EBA leaky spots, and the locations of EBA leaky spots and mitosis were found preferentially at sites of high shape indices which corresponded to regions of secondary flow. Around the orifices of intercostal arteries, endothelial nuclei morphology indicated a complex flow pattern, and mitotic cells and EBA leaky spots were found to exist in high frequencies. Characteristic 'V-shaped' topography of mitotic cells and EBA leaky spots around these orifices, similar to that of lipid accumulation in cholesterol-fed rabbits, can be explained by the local flow pattern. These findings show a correlation among the local hemodynamic pattern, endothelial ceil turnover and macromolecular leakage. The results are in support of the thesis that local hemodynamic factors may cause regional enhancements of endothelial cell turnover and permeability, which in turn lead to the focal nature of atherogenesis. (Supported by NHLBI Research Grant HL19454).

136

THE ASSOCIATION OF HEMODYNAMICS WTTH NTIMAL WHFTE BLOOD CELLS AND INCREASED LDL PERMEABILITY AT THE AORTO-CELIAC JUNCTION IN THE NORMAL-RABBIT

Richard A. Malinauskas, Kevin M. Barber, Robert A. Herrmann, Pasha Sarraf, and George A. Truskey Department of Biomedical Engineering, Duke University, Durham, NC 27708-0281

The objective of this study was to examine the relationship between arterial hemodynamics, endothelial cell (EC) nuclear orientation, increased EC permeability to radiolabelled low density lipoproteins (125I-LDL), and intimal white blood cells (WBC) at the normal rabbit aorto-celiac junction. Flow visualization studies using a $0.5 \mathrm{~mm}$ wide laser sheet to illuminate $75-90 \mu \mathrm{m}$ diameter particles were conducted in an exact transparent aorto-celiac model. Under steady mean physiologic flow conditions, paired spiral vortices on the order of $1 \mathrm{~mm}$ in diameter were localized to the lateral-distal flow divider region of the celiac orifice. The size of the vortices increased with the celiac flow rate and extended towards the dorsal wall of the aorta. Häutchen EC preparations were used to create en face aortic maps of the distributions of EC nuclear orientation, intimal WBC, including macrophages identified using RAM-11 immunostaining, and increased 125I-LDL permeability. Around the celiac artery branch, intimal WBC and sites of $125 \mathrm{I}$-LDL permeability were highest along the lateral-distal flow divider, where lesions preferentially originate in hypercholesterolemic rabbits. The EC nuclear shape and orientation were consistent with the time-averaged blood flow patterns, and were rounder with highly variable orientation along the flow divider. These results suggest that regions of time-averaged low wall shear stress are associated with intimal WBC and altered EC permeability in the normal rabbit and may have implications in atherogenesis.

137

SHEAR STRESS-INDUCED REORGANIZATION OF THE SURFACE TOPOGRAPHY OF LIVING ENDOTHELIAL CELLS IMAGED BY ATOMIC FORCE MICROSCOPY

Kenneth A. Barbee*, Peter F. Davies*, and Rameshwar Lal

*Department of Pathology and tDepartment of Medicine

University of Chicago, Chicago, IL

Hemodynamic forces affect endothelial cell structure and behavior. The three-dimensional shape of the apical surface of the endothelium will deternine the local flow pattems and therefore the distribution of shear stress acting on the cell membrane. The structure and compliance of both the membrane and the underlying cytoskeletal structure will determine the deformation responses and the distribution of forces transmitted throughout the cell. We have imaged the surface geometry and submembranous structures of living endothelial cells by atomic force microscopy. Cells in confluent monolayers were polygonal with smooth, rounded surfaces undulating with an amplitude of 2-4 $\mu \mathrm{m}$. Occasionally, a fine fibrous mesh was detected. Cells exposed to shear stress $\left(12 \mathrm{dyn} / \mathrm{cm}^{2}\right.$ ) for 24 hours were partially aligned, and the cells that had become elongated in the direction of the shear stress had height variations of less than $2 \mu \mathrm{m}$. In contrast to the unsheared cells, sheared cells exhibited a prominent surface structure characterized by aligned arrays of submembranous cytoskeletal fibers. An overall cell compliance was estimated by comparing images obtained at different imaging forces. In addition, a high resolution map of spatial variations in stiffness due to the presence of discrete cytoskeletal structures was constructed. This study represents the first observation of surface features in living endothelial cells and demonstrates significant changes in surface topography as a result of hemodynamic shear stress. 
138

\author{
DISTRIBUTJON OF ATHEROSCLEROTIC LESIONS IN THE HUMAN AORTA \\ Rong Xue, J. Fredrick Cornhill, Edward E. Herderick, Cynthia J. Roberts \\ Biomedical Engineering Center, The Ohio State University
}

Atherosclerosis is the most common disease that afflicts Western society. Preliminary investigations show that there is a very specific and well behaved topographical distribution on
localization of atherosclerotic lesions in the aorta of young people. An automated image processing system and statistical analysis techniques were developed to study the distribution processing system and stalistical analysis techniques were developed to study the distribution pattern of atherosclerotic lesions in the human thoracic and abdominal aorta.

The first study subjects were 106 males, blacks and whites, 15 to 29 years of age, who had died of violent causes. Techniques included image capture from color slides of the aot transformation of raw images to standard templates, image segmentation (lesion identification) computation of probability-of-occurrence maps and lesion percentage calculation. Regions of interest (boxes) were defined precisely on the probability-of-occurrence maps. The percentage of Iesion intimal surface and their mean values were calculated out within eact box and areas of high and low probability were identified. The linear regression model and the paired-i test were performed to investigate the symmetrical property of lesion distribution between the left and right halves of the human aorts.

Secondly, the spatial localization of both sudanophilic (fatty streaks) and raised lesions were characterized using similar image processing and statistical analysis techniques in the human thoracic and abdominal half aorta. Their inter-relationship were further evaluated. The study subject included 1378 males and females, blacks and whites, aged 15 to 34 years, who died of trauma. Raised lesions with high probability were demonstrated to be co-localized with region of high probability of fatty streaks in the abdominal aorta. However, not all regions of high probability of fatty streaks were associated with regions of high probability of raised lesions in young people.

\section{9}

A MODEL FOR THE GROWTH OF LDL LEAKAGE SITES IN ARTERIAL INTIMA Y. Huang ${ }^{1}, D$. Rumschitski ${ }^{2}$, S. Chien ${ }^{3}$ and $S$. Weinbaum ${ }^{1}$

Department of Mechanical ${ }^{1}$ and Chemical Engineering ${ }^{2}$, The City College of CUNY; ${ }^{3}$ AMES-

Biongineering, University of Califormia at San Diego

Recent experiments on the spread of HRP and LDL from cellular level endothelial leakage sites have revealed a remarkably rapid initial growth and subendothelial concentration levels that can not be explained by models in which the interstitial matrix of the intima and media are assumed to have nearly the same permeability properties, Yuan et al (1991). In this paper a new approach is adopted in modeling the intimal matrix. Direct observations of proteoglycan and collagen intimal matrix components obtained from the rapid freeze etching electron micrographs of rabbii intima by Frank and Fogelman (1989) are first used to determine the hydraulic conductivity and diffusivity of intimal matrix using random fiber matrix theory. This theory leads to the unexpected prediction that the permeability coefficients in the intima are nearly two orders of magnitude larger than in the media. These permeability properties are then used in a Brinkman equation to determine the lateral convective velocity in the intima in the vicinity of a leakage site. A combined convective-diffusive model for the intima intemal elastic lamina (IEL) and media is then solved to determine spot size growth and intimal concentration profiles in the vicinity of the leakage site. The resuls of this combined model reveal that HRP spots can growe vicinity of the dia af the leakage sites grow to roughly $3000 \mathrm{\mu m}^{2}$ after the few minutes and then do not spread further. The model also predicts that the average LDL concentration in the region of elevated concentration is roughly one half the lumen concentration and thus at least six fold higher than could be accounted for if one assumed that the available volume for LDL were similar to the measured ayailable volume for albumin in the intima. The latter predictions are in close agreement with the experimental observations of Truskey et al. (1992). Strong additional suppport for this clear dichotomy between intimal and medial matrix has been obtained from immunological studies with monoclonal antibodies which show that the matrix in the intima and the fenestral openings of the IEL differs markedly from medial matrix., Lark et al . (1987)

140

\section{ARTERIAL FLUID FLOW AND ENHANCED PERMEABILITY IN THE RABBIT AORTA Abdul I. Barakat and Clark K. Collon \\ Department of Chemical Engineering, MIT, Cambridge, MA}

We have previously reported the existence of sites within the normal rabbit aorta that are highly permeable to horseradish peroxidase (HRP). These sites have also been shown highly permeable to horseradsh peroxidase (HRP). These sites have also been shown to exhibit preferred spatial and angular orientations and to correlate to some extent with terotemic rabbits. In order to investigate whether or not the distribution of the HRP sites correlates with specific fluid mechanical phonomena, the detailed flow field in the rabbit aorta has been studied both ex-vivo using cinematographic techniques in natural aortas rendered transparent to allow flow visualzation and in-vivo using high resolution pulsed Doppler ultrasound velocimetry. The cinematographic studies in the thoracic aorta demonstrated the existence of induced clockwise helical flow along the ventral aspect of the aoric arch as well as replone of boundary layer separation along the lower curvature of the arch and at the proximal lipe of both arch branches. The region of tlow separation was occupied by a recirculation zone which periodically appeared and disappeared with flow pulsatility in the case of the brachiocephalic artery and by fonwand helical motion in the case of the lower curvature of the arch and the left subclavian artery. In the abdominal aorta, fluid streamines deflected oft the flow dividers of the major branches and reciculated arean the latel walls of the e thid mechanical in theractions, and consecuently more complex flow fields, were observed in the case of vessels in close anatomical proximity. The complex flow field in the aorta loads to sham spatial and temporal aracients in wall shear stress. The pulsed ine aorta vivo io shap spatial and temporal gracients in wall shear stress. The pulsed Doppler invivo studies demonstrated that anterial wall motion lends to decrease the extent of flow separation observed in the aorta and that flow near cortain ostia reverses direction (from the branch into the aorta) curing the dastolic phase of the cardiac cycle. This reverse flow has important implications on the resulling wall shear stress distribution in the aorta. The regarding similarities and diftenences are drawn.
141

LOW SHEAR, OSCILLATORY FLOW, AND ATHEROGENESIS: WHAT HAVE WE LEARNED FROM CELL CULTURE STUDIES?

Gabriel Helmlinger, Olivier Thoumine, and Robert M. Nerem Georgia Institute of Technology, Allanta, GA 30332

In vivo intimal thickening correlates with regions characterized hemodynamically as low shear. Such regions also exhibit oscillatory flow, and the enhanced intimal thickness is believed to represent a nidus for the early events leading to the development of atherosclerosis. Through cell culture studies much has been learned in the last decade about the influence of hemodynamics on vascular biology and the role of the endothelium in the early atherosclerotic events. Although most of such studies have been for steady flow conditions, our group has furned its attention in the last few years to a systematic investigation of pulsatile flow. These experiments have systematically studied reversing and non-reversing pulsatile flows, as well as purely oscillatory flow, all characterized by a $1 \mathrm{~Hz}$, sinusoidal waveform. Measurements have been made of cell morphology, properties of the extracellular matrix, and signaling mechanisms. From initial results it is now clear that endothelial cells express differences due to these different pulsatile flow environments. Most notably, an endothelial monolayer in the presence of a purely oscillatory flow, with no mean flow component, is not the same as a similar monolayer for a static, no-flow condition. This suggests that in vivo the vascular endothelium in oscillatory flow regions may possess different characteristics from those exhibited in regions of stasis.

\section{2}

END-STAGE CLINICAL ATHEROSCLEROSIS: ROLE OF HEMODYNAMICS IN THE RUPTURE OF THE PLAQUE CAP

David N. Ku, J. *Micah Downing, Barbara McCord and Sophie Biz. Georgia Institute of Technology, Atlanta, GA, *Armstrong Laboratory/OEBN, WrightPatterson AFB, OH

Advanced human atherosclerosis is characterized by a high-grade stenosis covered with a fibrous plaque cap. The end-stage event usually comes when the plaque cap ruptures and exudes the contents of the atheroma into the bloodstream. The plaque rupture produces local thrombus occlusion or embolization of the contents downstream. The mechanism for rupture may be a direct consequence of the unusual hemodynamics associated with the highgrade stenosis. We have shown that the low fluid pressures in the throat can cause a collapse of the arterial wall, both theoretically and experimentally. This collapse subsequently leads to strong oscillations in wall motion, high frequency compression of the fibrous cap, and choking of the flow rate through the stenosis. The plaque cap can fatigue and eventually fracture from these unusual loading conditions. This mechanical mechanism of plaque rupture provides a new explanation for the catastrophic clinical consequences of end-stage atherosclerosis. 
143

DISTRIBUTION PATTERNS OF ATHEROSCLEROTIC LESIONS AND ENDOTHELIAL INIURY IN AORTA.

Knut Pettersson, Cardiovascular Pharmacology, Astra Hăssle AB, Mờndal, Sweden.

Atherosclerosis is not unifomly distributed over the anterial surface. The preferential location to areas close to branching points and curvatures suggests that hemodynamic strain may be involved in the initiation of atherogenesis Virchow (1856). Endothelial cells in predilection sites for atherosclerosis often lose their capacity to exclude anionic dyes, used in cell culture for evaluation of cell viability. These cells also contain $\operatorname{lgG}$, and the presence of IgG could be used as a marker for endothelial injury. IgG-containing endothelial cells in culture were detached from the endothelial mono-layers while beeing undermined by surrounding cells, leaving no defect in the endothelium. These cells can thus be used as markers for non-denuding injury in the endothelium

In normal rabbits, few injured cells are found in the descendent thoracic aorta, except for the areas around the ostiae of branching intercostal arteries, where the occurrence of injured cells is 30 -fold higher. This iocalisation corresponds to where the first intimal lesions occur in response to an atherogenic diet. We have observed that risk factors for atherosclerosis also cause non-denuding endothelial injury, e.g. smoking, hypercholesterolemia and sympathetic activation. We suggest that this form of endothelial injury can be a localizing, if not initiating, factor for atherogenesis. The distribution of such injury to arterial branch points and curvatures suggests that hemodynamic factor(s) can cause such injury, thereby leading to the development of atherosclerosis.

144

Mechanical Activation of Endothelial Cells

Scott L. Diamond, Fred Sachs, and Wade J. Sigdurson

Depts. of Chem. Engr. and Biophysics, S.U.N.Y., Buffalo, NY 14260.

Measurements of intracellular calcium were conducted in furst passage human umbilical vein endothelial cells (HUVEC) exposed to laminar fluid shear stress or mechanical perturbation using a micropipette. Gentle pushing on an individual cell using the side walls of a micropipette caused transient increases in calcium each time the cell was poked ( $\mathrm{n}>3$ pokes in over 27 cells using 5 separate cell harvests). Some cells responded more than 6 times without dye leakage or membrane blebbing. Gentle pushing on the cell did not cause membrane puncture as indicated by (i) the absence of rapid dye leakage, (ii) the regulated nature of the calcium response, and (iii) the absense of membrane blebbing. Similar responses were seen in passaged bovine aortic endothelial cells. Using positive pressure of 50 to $100 \mathrm{~mm}-\mathrm{Hg}$ in a micropipette, we created very narrow fluid streams (with wall shear stresses of -0.001 to $0.1 \mathrm{dyne} / \mathrm{cm}^{2}$ ) tangentially over the cells. However, these low shear streams had no effect on intracellular calcium. Interestingly, normally impinging flows which caused slight depressions of the cell surface induced a calcium mobilization. The firs poke-induced change in calcium was not affected by the stretch channel blocker gadolinium $\left(\mathrm{Gd}^{3+}\right)$, or by low extracellular calcium $(10 \mu \mathrm{M})$, although subsequent poke-induced calcium transients were greatly attenuated by these actions. In the absense of extracellular calcium, however, the ability of HUVEC to respond to the first poke was not present, indicating the requirement of some extracellular calcium as a mediator of signalling or as the major source of calcium during mobilization.

145

MECHANO-SIGNALING IN VASCULAR CELLS EXPOSED TO CYCLICSTRAIN Ira Mills and Bauer E. Sumpio

Dept. of Surgery, Yale University School of Medicine, New Haven, CT

Endothelial cells (EC) subjected to cyclic strain exhibit marked changes in morphology, proliferation and secretion of macromolecules including endothelin, prostacyclin and tissue plasminogen activator (t-PA). We have begun to delineate the signal transduction pathways that may be involved in coupling mechanical forces to cellular responses. Acute cyclic strain causes activation of phospholipid turnover leading to the production of both inositol phosphates and diacylglycerol in EC. The elevation of inositol phosphates is an acute, monophasic response whereas generation of diacylglycerol production is biphasic and sustained. These events most likely reflect activation of both phospholipases $C$ and D. Biphasic stimulation of diacylglycerol formation by cyclic strain is associated with biphasic activation of protein kiuase $\mathrm{C}$ in $\mathrm{EC}$. Activation of protein kinase $\mathrm{C}$ in $\mathrm{EC}$ by cyclic strain constitu ted a translocation of enzyme activity from a cytosolic to a membrane fraction. Strain-induced protein kinase $C$ membranous activity was primarily found in the perinuclear region of EC. Adenylate cyclase is also elevated acutely by cyclic strain in EC. Cyclic strain-induced activation of adenylate cyclase is associated with the transien loss of inhibitory $G$ protein $\left(G_{i x, 2,2}\right)$ immunoreactivity that is thought to involve a posttranslational protein modification at the carboxyl terminus of the protein. In addition, cyclic strain-induced activation of adenylate cyclase leads to the accumulation of cyclic AMP and activation of protein kinase A in EC. These data support the hypothesis that cyclic strain activates both the adenylate cyclase/cyclic $A M P$ /protein kinase $A$ and the $\mathrm{Ca}^{2+} /$ phospholipid/protein kinase $\mathrm{C}$ signal transduction patbways in EC. Recent studies demonstrate that cyclic strain causes the generation of transcription factors AP-1, NF-kB and CREB in EC. Ongoing studies in our laboratory are being performed to delineatc the coupling between mechanical perturbation and specific gene activation in EC.
146

PRESSURE INDUCED WALL STRESS AND ATHEROSCLEROSIS IN THE CAROTID ARTERY BIFURCATION

Robert S. Salzar ${ }^{\dagger}$, Mano J. Thubrikar $r^{*}$ Richard T. Eppinkt

Department of Civil Engincering and Applied Mechanics, The University of Virginia, Charlottesville, Virginia

Heineman Medical Research Laboratories, Carolinas Medical Center, Charlotte, North Carolina

A possible correlation between the locations of atheroscleretic lesions in the carotid artery bifurcation and the regions of tissue experiencing high pressure induced wall stress was investigated. Six cadaver specimens were obtained to determine the common areas of investigated. Six cadaver spocimens were obtained to determine the common areas of element analysis models, both the quantitative, as well as the qualitative behavior of the artery under internal pressure was investigated. In all six carotid specimeas, two distinct areas consistently exhibited substantial lesion growth. The most severe lesions occurred along the sinus bulb, with lesions ranging from a general thickening of the artery wall to the almost total blockage of the jumen. The second area was at the point of bifurcation (crotch), where the lesions were highly localized and did not appear to threaten the blood flow. Through careful measuremeats of two representative artery specimens, including the overall dimensions as well as wall thicknesses, two finite element models were created. By applying the in vivo equivalent internal pressure as well as boundary conditions to the models, two areas of elevated pressure induced stress were discovered. The highest stress concentration occurred at the point of bifurcation, with stresses 9 to 14 times the first principal stress in the common carotid. This bifurcation, with stresses 9 to 14 times the first principal stress in the common carotid. This
stress was distributed over a very small region of the model. The other area of high stress occurred along the sinus bulb. This stress was 3 to 4 times that of the common carotid, but was distributed over a much greater area, encompassing the whole of the sinus bulb. Thus, there appears to be a correlation between both the magnitude and distribution of high wall stress and the locations of atherosclerotic lesions.

147

\section{SIMULTANEOUS STRAIN AND MORPHOLOGIC MEASUREMENTS IN HUMAN AORTIC CROSS-SECTIONS \\ R. VITO* ${ }^{*}$ S. GLAGOV**, C. XU ${ }^{* *}$, M. WHANG*, D. BEATIE* *GA. INSTITUTE OF TECHNOLOGY, **U. CHICAGO}

The influence of mechanical factors such as the state of strain on the adaptation of the vessel wall may be related to atherosclerosis. In this work, we examine mechanical and histologic correlates in cross-sections of human thoracic and abdominal aorta obtained at autopsy. In previous studies of vascular mechanics, the distribution of strain through the thickness is assumed axis-symmetric and the wall homogeneous. However, it is well known that variations of microstructure occur both within a cross-section and along the aortic tree. We measured the deformation of $5 \mathrm{~mm}$ long segments of human aorta by tracking $10 \mu$ selfadherent particles scattered through the thickness at posterior, anterior and side locations. The segments are placed over a cylindrical latex tube which is inflated so that pressure exerted on the vessel lumen is in the physiologic range. Specimens are then pressure fixed and prepared for light microscopy. Several correlations were observed. In particular, the average peak strain was inversely correlated with the intimal area fraction which could imply a role for strain level in intimal thickening.

\section{8}

PROTECTIVE ADAPTATION TO CHRONICAL MECHANICAL INJURY AS THE ETIOLOGY OF ARTERIAL OCCLUSIVEDISEASE

Kenneth R. Kensey ${ }^{1}$ and Young $t$. Cho ${ }^{2}$

1. Kensey Nash Laboratories, Exton, PA

2. Dept. of Mechanical Engineering, Drexel University, Philadelphia, PA.

The aim of this investigation is to identify one underlying cause for arterial occlusive disease. Presented is a hypothesis implicating an inherent protective adaptation process of the arterial wall 10 mechanical injury as the initiating event for arterial occlusive disease. Computer simulation and historical human pathological data are used to support this hypothesis. An amplified protosystolic pulse (AMPPULSE) is compared to a less amplified pulse to quantitatively study the effect of AMPPULSE circulation on arterial injury patterns. Most commonly observed clinical presentations of atherosclerosis previously not explained nor understood can be explained using the present protective adaptation hypothesis. In general, to avoid rupture artery walls adapt to excessive tensile stress by thickening and stiffening, thus reducing vessel compliance and lumen size. Consequently, shear stresses in site-specific areas increase in magnitude at high shear regions and oscillale in directions more rapidly in low shear regions. It appears that the two types of shear stress cause injuries to the intima under amplified protosystolic pulse (AMPPULSE) circulation. Adapting to this injury, AMPPULSE circulation occurs and the heart must work excessively to circulate a particular blood volume. A vascular system that requires excessive work by the heart to perfuse has a high propensity for injury and thus eventual occlusion. 
149

ENDOTHELLAL ORIENTATION AS A REFLECTION OF STRESS

J.D. Deck

Department of Anatomy and Cell Biology, University of Virginia Health Sciences Center, Charlottesville, Virginia 22908.

Vascular endothelial cells are generally thought to be aligned with the long axis of the vessels they line by shear stresses associated with blood flow. One would expect the endothelium of aortic valve leaflets to be particularly affected by the surge of systolic blood llow. Inspection of leaflet sufaces with the scanning electron microscope shows, however, that endothelial cells are aligned circumferentially on the leaflet, parallel to its free edge and perpendicular to the direction of blood flow. Endothelial cells on the aortic face of the leaflet are stretched out along the circumferential ridges of fibrous tissue which otherwise characterize this surface. Except over the leaflet nodule near the center of the leaflet's free edge, the same orientation of endothelial cells is found on the ventricular face of the leaflet, the surface first encountered by ventricular outflow. It therefore appears that shear stress acting on the leaflet endothelial cells is countered by other, stronger forces, such as the stress of leaflet closing and suspension during diastole, which stress seems to dictate the directionality of principal fibrous tissues within the leaflet. It would be interesting to observe whether endothelial cells at arterial brane leaflet. also subject to differing types of stresses, likewise depart from the expected longitudinal orientation.

150

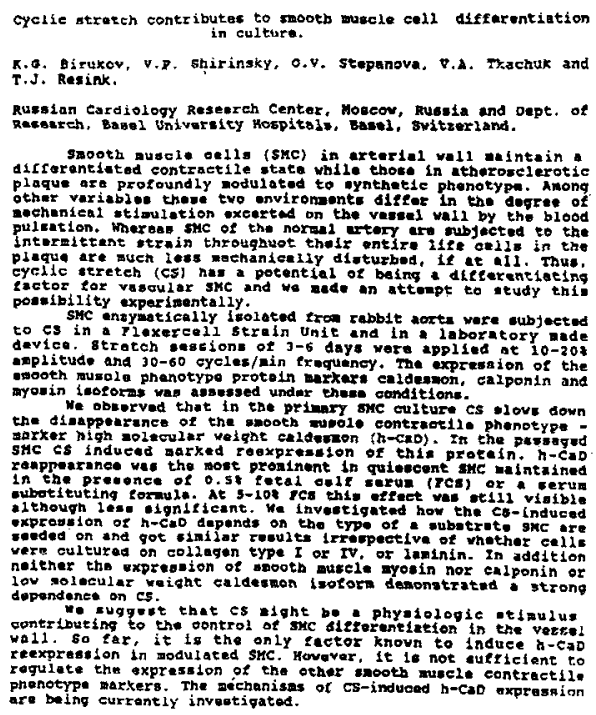

151

ABSENCE OF ATHEROSCLEROSIS IN INTRAMYOCARDIAL CORONARY ARTERIES AND ITS RELATIONSHIP TO MURAL STRESS.

Francis Robicsek, M.D., Mano J. Thubrikar, Ph.D.

Heineman Medical Research Laboratory, Carolinas Medical Center, Chariotte, N.C.

The occurrence of atherosclerotic lesions was studied in both epicardial and intramyocardial coronary arteries in 150 patients undergoing coronary bypass surgery. Most patients showed diffuse coronary arteriosclerosis. In 17 patients the circumflex coronary patients showed diffuse coronary arteriosclerosis. In 17 patients the circumflex coronary
artery gave off branches whose distal portions ran entirely intramyocardially. Of these, 15 had no atherosclerosis in the intramyocardial branch whereas the same anteries epicardially had lesions, 1 had a calcified pleque, and 1 had a recanalized thrombus. To study this phenomenon further intramyocardial pressures (IMP) were measured in 8 canine hearts. The pressures were measured with a micro-tip catheter which had a tiny recessed pressure sensor. The IMP was measured on the lateral wall of the left ventricle ( $\mathrm{LV}$ ) and at the apex. At each location the pressure was measured within the myocardium at 3 depths: Near the epicardium, in the mid wall, and near the endocardium. The myocardium developed significant pressures within the muscle mass throughout the cardiac cycle. The IMP in the epicardial region was the lowest, in the mid wall it was higher, and in the endocardial region it was the highest. Subepicardial pressures were as high as $30-80 \%$ of the LV cavity pressure which was also measured simultaneously using a fluid filled catheter. Subendocardial pressures were generally higher than the LV cavity pressures. High IMP indicates that the pressure gradient across the intramyocardial coronary artery wall is significantly reduced, and sometimes the gradient may even be eliminated or reversed. Hence, the intramyocardial arteries experience less pressure gradient and therefore lower mural stress compared to the epicardial arteries. In conclusion, the absence of atherosclerosis in the intramyocardial coronary arteries appears to be due to reduced mural stress, i.e. the lowering or absence of the transmural pressure gradient.
152

ARTERIAL TISSUE MECHANICS AND ATHEROSCLEROSIS Renu Virmani, M.D.

Armed Forces Institute of Pathology

A comparative morphologic study of aortic changes with aging will be presented in different populations with an attempt to separate the effects of hypertension and atherosclerosis. chinese and hypertension and atherosclerosis. Chinese and to have a high prevalence of hypertension and atherosclerosis, respectively. Aging resulted in an increase in aortic circumference with the greatest increase in ascending aorta in the Chinese and in the abdominal aorta in the occidental. Intimal thickness increased with age and was maximal in the abdominal aorta and was greater in the occidental population. In the Chinese population, isolated systolic hypertension resulted in greatest increase in the ascending aorta whereas idiopathic systolic hypertension caused the largest increase in the abdominal aorta. Intimal thickness was less in ascending aorta in isolated systolic hypertension and greatest in essential hypertension in abdominal aorta. These morphologic changes may account for the finding of increased aortic pulse wave velocity observed with advancing age and may be responsible for the systolic hypertension seen in aging population.

153

PSYCHOSOCIAL INFLUENCES ON ATHEROSCLEROSIS: EFFECTS AND MECHANISMS IN NONHUMAN PRIMATES.

J.R. Kaplan and S.B. Manuck

Department of Comparative Medicine, Bowman Gray School of Medicine of Wake Forest University, Winston-Salem, NC 27157-1040

Psychosocial factors are often implicated in the pathogenesis of human atherosclerosis and coronary heart disease (CHD). Experimental manipulation of animal models has shown that both environmental perturbations and individual behavioral characteristics potentiate atherogenesis and, occasionally result in manifestations of CHD. The data to be presented here provide initial evidence concerning the influence of behavioral factors on atherosclerosis as well as concerning the pathophysiological mechanisms or processes mediating such behavioral effects. These data support four preliminary conclusions: 1) psychosocial factors influence atherosclerosis and impair vascular responsiveness in monkeys, two processes that contribute to the development and expression of CHD in humans; 2) clear analogies exist between the psychosocial factors that potentiate atherogenesis and vasculat dysfunction in monkeys and those that are believed to influence the development of CHD in humans; 3) recurrent activation of the sympathetic nervous system is likely the common patbway mediating the influence of various psychosocial factors on vascular abnormalities and CHD in monkeys and, hence, possibly also in humans; and 4) such sympathetic nervous system activation apparently damages the coronary arteries via hemodynamic or kinetic rather than metabolic (systemic) factors.

154

DETERMINANTS OF MECHANICAL PROPERTIES OF ATHEROSCLEROTIC ARTERIES Professor G.V.R. Born,

The William Harvey Research Institute,

St. Bartholomew's llosnital Medical College,

Charterhouse Square London, FCIM 6BQ.

One determinant is calcium. which increases with age in normal arteries and accumulates excessively in atherosclerotic arteries. Another determinant is connective tissue which thickens the intima. Elastin has opposing effects: it contributes to stiffening through association with t,Dr, whereby hydrating water is decreased, and to so tening through disintegration in the internat elastic lamina. Thickening and hardening decrease elasticity and distensibility and increase pulse wave velocity. Recent analyses have elucidated the dependence of plaque fissure (the immediate cause of coronary thrombosis) on the mechanical, biochemical anc cellular properties of the lesions. 
155

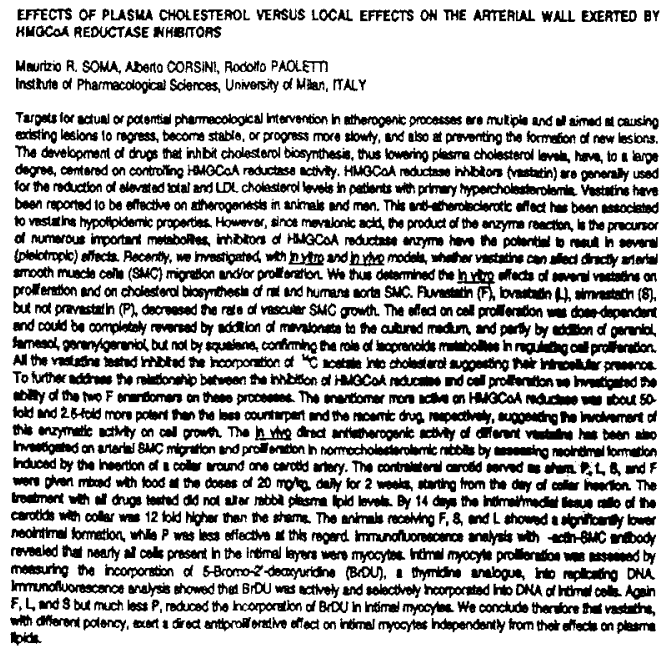

156

PATHOPHYSIOLOGICAL ALTERATIONS OF CORONARY MICROVASCULAR RESPONSES IN ATHEROSCLEROSIS

Lih. Kuo, William M. Chilian, and Michael J. Davis.

Department of Medical Physiology and Microcirculation Research Institute, Texas A\&M

University Health Science Center, College Station, TX 77843.

Atherosclerosis is known to alter the mechanical properties and pharmacological reactions of large conduit arteries. Recent studies suggest that such changes may also occur in microvessels which do not exhibil anatomical lesions. In this study, we hypothesized that in addition to pharmacological alterations, cndothelium-dependent vasomotor responses to a physiological stimulus, flow, are also altered in coronary resistance arterioles downstream from atherosclerotic lesions. To test this hypothesis, subepicardial arterioles $(40-70 \mu \mathrm{m})$ from normal and atherosclerotic pigs were isolated, cannulated with micropipettes and connected to two independent reservoir systems. Initially, the cannulated vessels were set to the baseline condition, $60 \mathrm{cmH}_{2} \mathrm{O}$ intraluminal pressure (IP) without flow, for development of spontaneous tone. Pharmacological responses of these vessels to the endothelium-dependent agonists, serotonin (5HT), histamine and ADP, were assessed by studying the dose-diameter relationships. Vascular responses to physiological stimuli, flow, were assessed by studying the flow-diamete relationships. Flow was initiated by simultaneously moving the reservoirs in equal but opposite directions without changing IP. In arterioles from normal pigs, 5HT, histamine, and ADP produced dose-dependent, endothelium-mediated dilation. Flow-induced dilation was only observed in vessels with a functional intact endothelium. Conversely, in arterioles from atherosclerotic pigs, endotheiitum-dependent dilation to 5HT, histamine, ADP, and to flow was attenuated, but nicroprusside produced equal vasodilation of arterioles from both groups. L arginine $(3 \mathrm{mM})$, a thological precursor for nitric oxide synthesis, restored the impaiment of endothelium-dependent vasodilatory function in response to $5 \mathrm{HT}$, histamine, ADP and flow in vessels from atherosclerotic pigs. We speculate that the synthesis and/or release of nitric oxide from L-arginine in coronary arterioles downstream from atherosclerotic lesions is impaired, altering pharmacological reactivity of the arterioles to endothelium-dependent agonists and compromising flow-induced vasodilation.

\section{7}

HEMODYNAMIC FORCE-INDUCED DIFFERENCE OF INTERENDOTHELIAL JUNCTIONAL COMPLEX OF RABBIT AORTA

Yoshida Y, Okano M, Wang S, Kobayashi M

Department of Pathology, Yamanashi Medical University

It has been proposed that hemodynamic forces play important roles on localization of atherosclerosis. We reported that at surgically coarctated abdominal aortas of cholesterol-fed rabbits, endothelial cells (ECs) were long fusiform at the coarctation, which were nonsudanophilic and expected to be exposed to laminar and high shear stress. On the other hand, ECs were ellipsoidal downstream from the coarctation, which were sudanophilic and imposed by low shear stress. In this regard, to reveal why the difference of EC permeability could occur. We compared the morphology of tight (TJ) and gap (GJ) junctions of $\mathrm{EC}$ in regions of interest using a freeze-fracture method. Surgically coarchated abdominal aortas of normo- and hyperlipidemic rabbits were fixed by infusion of glutaraldehyde and cut into small pieces from [1] - [7] regions and the uncoarctated abdominal aortas $[8]$ [9] as controls. TIs consisted of both continuous (zonular type) and focal, discontinuous (macular type) strands with free ending elements. The zonular / macular ratios were $1.91-2.86$ at [1][2][8][9], maximal at [3] (3,00-420), significantly small at [6] $(0.29-0.35)$ and [7] (0) $0.58)$. At [4] and [5] regions, TJs were scarce with some macular types. The average numbers of strands at [3] regions $(1.89-1.96)$ were larger than others. GJs were classified into two types: regular, with smooth perimeters, and irregular, characterized by bizarre shapes. The regular / irregular ratios at [5] $(0.64-0.70)$ and [3][4][6][7] $(0.80-2.00)$ were significantly less than [1][2][8][9] (2.56 - 5.60). It might be concluded that low shear stress modulates the ultrastructure of junctional complexes to become more permeable to macromolecules and subsequently to bring vulnearable properties to the vessel wall in atherogenesis.
158

ANTIOXIDANT-, CA ${ }^{++}$ANTAGONISTIC-, AND ANTI-HYPERINSULINEMIA PROPERTIES OF AMLODIPINE SUPPRESS ATHEROSCLEROSIS RC Sharma, DM Kramsch, HN Hodis, CR Liu, G Izumi, P Liu, and A Sevanian.

University of Southern California, Los Angeles, CA, USA

Site-specific progression of atherosclerosis was assessed in aorta of 26 cynomolgus monkeys at baseline, 6 and 12 months using B-mode duplex pulsed Doppler: 10 monkeys fed a highbutter atherogenic diet $(A D), 11 \mathrm{AD}$ monkeys treated with 2 $\mathrm{mg} / \mathrm{Kg}$ body weight amlodipine (ADA) and 5 control animals (standard chow). Both AD groups showed comparable rises in (mg/dl mean $\pm \mathrm{SD}$ ) total cholesterol (C), LDL-C and triglycerides (AD: $487+180,308 \pm 129,109 \pm 81$; ADA $403 \pm 117,286$ $\pm 93,75 \pm 19$, respectively). Plasma oxidized LDL-C (LDL) and fasting insulin (I) also rose significantly from baseline in $A D$ monkeys (LDL ${ }^{-}$: 1.8 to 11.1 ; 1 : 19.9 to 36.5 ) but not in ADA animals $(\mathrm{p}<0.001)$. Arterial stiffness index beta increased significantly while blood flow arrival time decreased significantly in the AD but not the ADA group. At sacrifice AD monkeys showed marked atherosclerosis in aorta as well as in coronary arteries. By contrast, in ADA atherosclerosis was absent in 5 animals, mild in 4 and marked only in 2 monkeys. conclusion: amiodipine, by preventing LDL oxidation and hyperinsulinemia, appears to intensify atherosclerosis suppression of the $\mathrm{Ca}$ channel blocker without reducing elevated plasma levels of total $\mathrm{C}$ and LDL-C.

\section{9}

APPLICATION OF A MICROSTRUCTURAL DAMAGE THEORY TO MODEL ARTERIAL ANEURYSM FORMATION

David A. Vorp, KR Rajagopal, M.L. Raghavan, and Marshall W. Webster

University of Pinsburgh, Deparment of Surgery, Pitsburgh, PA 15261

A marked disparity is found in the microstructure of the wall of arterial aneurysms from that of the nonaneurysmal portion of the vessel, suggesting differences in the elastic properties. We have adapted a recent theory describing the constitutive behavior of a nonlinearly elastic material undergoing deformation-induced microstructural damage to model the changing mechanics of the undergoing deformation-induced microstructural damage to model the changing mechanics of the arterial wall as it dilates. The theory is based on the idea of "elastic mixtures" to describe
inelastic deformations. Assume that a scgment of artery undergoes a deformation that satisfies inelastic deformations. Assume that a segment of artery undergoes a deformation that satisfies original material and a newly formed material. Assuming that both behave as "power-law neoHookean" materials, the Cauchy stress $T_{i j}$ within the elastic mixturc is given as

$$
T_{r}-p \delta_{1}+\xi \mu\left[1+\frac{b}{n}\left(l_{1}-3\right)\right]^{n-1} B_{l 0}+(1-\xi) \mu\left[1+\frac{b}{n}\left(l_{1}-3\right)\right\}^{n-1} B_{k 1}
$$

where $p$ is the hydrostatic pressure, $\xi$ is the mass fraction of the original material still present in the elastic mixture, and $\mu, b, n$ and their "hatted" counterparts are elastic constants of the original and newly formed materials, respectively, $B$ is the left Green-St.Venant stretch tensor, and $I_{1}$ its first invariant. Subscripts ' $o$ ' and ' 1 ' refer to the configuration of the original reference (stressfrrst invariant. Subscripts 'o' and 'Tefer to the configuration of the original reference (stress-
free) state and when the new material is formed. Twenty-six (26) tissue specimens were obuined during elective repair of aneurysms of the abdominal aorta, thoracic aorta, popliteal artery, on iliac during elective repair of aneurystrs of the abdominal aorta, thoracic aorta, popliteal artery, or iliac
artery. These were loaded under uniaxial tension in an Instron ${ }^{2}$ machine providing stress-stretch artery. These were loaded under uniaxial tension in an Instron ${ }^{\text {m }}$ machine providing stress-stretch
data pairs. The model parameters were obtained using nonlinear regression asalysis on the data pairs. The model parameters were obtained using nonlinear regression analysis on the
experimental data. For all specimens, the deformation at which irreversible damage occurred was experimental data. For ali specimens, the deformation at which irreversible damage occurred was
also determined. Our results uphold the hypothesis that marked mechanical differences exist also determined. Our results uphold the hypothesis that marked mechanical differences exist
between the normal and aneurysmal tissue. Further, we demonstrate that our model makes it possible to predict a critical stress level (or diameter) at which irreversible microstrucnural damage, and hence aneurysm formation, will occur. 
160

ENHANCED MCP. 1 AND VCAM-1 EXPRESSION IN HUMAN AORTIC ENDOTHELIAL CELLS EXPOSED TO PROLONGED LOW SHEAR STRESS

Eugene A. Sprague

University of Texas Health Science Center, San Antonio, Texas 78284

Enhanced monocyte recruitment is a hallmark of focal arterial areas prone to develop atherosclerotic lesions. These sites are coincident with sites of low hemodynamic shear stress. Using a parallel plate flow system, we have previously demonstrated that cultured bovine aortic endothelial cells preconditioned to prolonged low shear (LS, 0.5 dynes $/ \mathrm{cm}^{2}$ ) in vitro exhibit significantly increased monocyte recruitment and adhesion relative to cells preconditioned to either high (HS, 30 dynes $/ \mathrm{cm}^{2}$ ) or no shear (NS)stress. In the present studies, cultured human aortic cells (HAEC) were preconditioned to LS, HS, or NS for $48 \mathrm{~h}$. After preconditioning, HAEC were either fixed for immunocytochemistry or extracted for RNA isolation and analyses. Northern blot analyses of total cellular RNA revealed that HAEC exposed to LS for $48 \mathrm{~h}$ exhibit a greatly enhanced expression of mRNA for both monocyte chemotactic protein (MCP-1) and vascular cell adhesion molecule (VCAM-1) relative to HS and NS exposed HAEC. Immunocytochemical analyses confirmed increased VCAM-1 protein on LS treated cells. These results suggest a mechanism through which hemodynamic flow pattern may influence the focal initiation and distribution of atherosclerosis.

161

REGULATION OF ENDOTHELIAL CELL MIGRATION BY PLASMA LIPOPROTEINS Fox, P.L., Chisolm, G.M., Murugesan, G., and Sa, G.

Department of Cell Biology, Cleveland Clinic Research Institute, Cleveland, OH 44195.

Endothelial cell migration is a critical and initiating event in the formation of new blood vessels, in the repair of injured vessels, and in the endothelialization of synthetic vascular grafts and other implants. We have investigated the role of lipoproteins in regulating the woundhealing response of vascular endothelial cells (EC) in vitro. Confluent cultures of bovine aortic healing response of vascular endothelial cells (EC) in vitro. Confluent cultures of bovine aortic as the number of cells and mean distance. Compelling evidence suggests that oxidized low density lipoprotein (oxLDL) is present in atherosclerotic lesions, but its role in lesion formation has not been defined. Oxidized LDL reduced migration up to $75 \%$ in a dose- and
oxidation-dependent manner. The inhibition was not due to cell toxicity since the inhibition of oxidation-dependent manner. The inhibition was not due to cell toxicity since the inhibition of migration was completely reversed $16 \mathrm{~b}$ after removal of the lipoprotein. The inhibitor molecule was shown to be a lipid since organic solvent extracts inhibited migration to nearly the same extent as the intact particle. These data suggest that oxLDL may limit the bealing response of endothelium after a wound injury. We also examined the effect of plasma bigh density lipoprotein (HDL) on migration of EC in vitro. Physiological levels of HDL increased EC migration by up to $100 \%$ compared to controls. At an optimal concentration, HDL was at least as stimulatory as basic fibroblast growth factor (FGF), and siger consistent with a signa tran consistent with a signal transdluction mechanism. Treatment of EC with optimal doses of both dictive agents utilize discinct signaling palhways. To deternine the chemical nature of the stimulatory molecule, all was fraction contained essentially all of the prom slightly stimulatory. The promigratory activity of HDL may accelerate regeneration of the endothelium after a denuding injury in vivo. These results suggest novel mechanisms by which lipoproteins may be eicher causative or protective agents in the development of cardiovascular disease.

162

\section{WALL SHEAR STRESS AND GRAFT ORIENTATION ON INTIMAL} HYPERPLASIA IN TAPERED GRAFTS

"David N. Ku, Tarek A. Salam, Alan B. Lumsden, William D. Suggs, "Georgia Institute of Technology and Emory University. Atlanta, GA

Vascular grafts often fail from the development of cellular stenoses at the anastomotic ends. The smooth muscle cell thickening may be stimulated by the local hemodynamic stresses, both in magnitude and direction. This study was designed to evaluate the effect of wall shear stress and direction of graft taper of anastomotic neointimal hyperplasia independent of flow rate in tapered polytetrafluoroethylene (PTFE) grafts. Twenty 4-7 min tapered PTFE grafts were inserted in the femoral and carotid locations bilaterally in an end-to-side fashion in five mongrel dogs. The grafts were harvested after 16 weeks using a pressure perfusion technique. Mean thickness of anastomotic neointimal hyperplasia was measured by computer image analysis.

At the time of implantation, the calculated mean shear stress averaged $18 \mathrm{dyne} / \mathrm{cm}^{2}$ at the $4 \mathrm{~mm}$ end and $3.4 \mathrm{dyne} / \mathrm{cm}^{2}$ at the $7 \mathrm{~mm}$ end $(p<0.0001)$. Seventeen of twenty grafts were patent at the time of harvest. Mean anastomotic neointimal thickness was significantly greater at the $7 \mathrm{~mm}$ end compared to the $4 \mathrm{~mm}$ end of these grafts $(0.29 \mathrm{~mm}$ vs. $0.18 \mathrm{~mm}, p<0.05)$. The amount of intimal thickening can be statistically correlated to the inverse of the local mean shear rate. This data shows that the development of thicker anastomotic neointima is primarily governed by low wall shear stress in tapered PTFE grafts even with varying flow conditions and orientation.
163

GROWTH FACTOR IMMOBILIZATION ONTO BIOMATERIALS TO INDUCE TRANSMURAL ANGIOGENESIS AND ENDOTHELIALIZATION

HP Greisler, DU Kim, JW Winkles, and WH Burgess

Endothelial recruitment to the blood-contacting surface can theoretically be enhanced by immobilization of specific endothelial cell chemoattractant and mitogenic substances. We have evaluated several immobilization schemes and find optimal in vitro and in vivo efficacy using fibrin glue (FG) suspension containing FGF -1 and heparin. These suspensions were impregnated into $60 \mu$ internodal distance ePTFE grafts $30 \mathrm{~cm} \times 8 \mathrm{~mm}$ ID. Two control groups were either untreated or treated with FG without FGF-1. Grafts were implanted into canine thoracoabdominal aortas for 10,30 or 140 days ( $n=3 /$ time/group) and harvested 10 hours IM injection of tritiated thymidine $(0.5 \mu \mathrm{Ci} / \mathrm{kg})$. Specimens were studied histologically and by autoradiography. Inner capsule collagen was quantitated and reverse transcriptase-PCR used for detection of al tered gene expression for FGF-1, FGF-2, TGF- $\beta_{1}$, FGF receptor-1. Results showed no thromboses or anastomotic intimal hyperplasia. Abundant transmural angiogenesis was present by one month in the experimental group yielding a confiuent endothelialized surface present also at 20 weeks, not seen in either negative control group at any time point. Cross-sectional autoradiography showed an increased mitotic index at 30 days in the FGF-1 group only, returning to baseline at 140 days. Inner capsule thickness and total collagen content did not significantly differ among the groups at any single time point. No FGF-1 induced modulated gene expression was found. The application of growth factor affixation to biomaterials surf aces may promote spontaneous endothelialization in man.

\section{4}

IN SITU PHOTOPOLYMERIZATION OF THIN HYDROGEL FILMS FOR PREVENTION OF THROMBOSIS AFTER VASCULAR INJURY JL Hill-West, SM Chowdhury, AS Sawhney, CP Pathak, MJ Slepian, JA Hubbell Department of Biomedical Engineering, University of Texas, Austin, TX 78712

In situ photopolymerization of very thin biodegradable, hydrogel films on the lumen surface of injured rat carotid arteries significantly reduces thrombosis and increases long-term vessel patency. Films were formed by interfacial polymerization of a water-soluble, biodegradable, diacrylated polyethylene glycol (PEG) derivative within an artery which had the photosensitive dye eosin $\mathrm{Y}$ adsorbed to its lumen surface. Polymerization occurred upon illumination with an argon ion laser at low intensity, producing a cross-linked hydrogel Environmental scanning electron microscopy (ESEM) revealed the thickness of these films to be approximately $10 \mathrm{\mu m}$, and the films to be adherent to the vessel wall following exposure to prolonged blood flow. This material degrades in vivo within several days. These thin films function as biocompatible mechanical barriers to platelet interactions with the injured vessel. Platelets do not adhere to PEG as it is very resistant to cell adhesion and protein adsorption. Efficacy in locally preventing thrombosis following a vessel injury was evaluated in a rat carotid artery following crush injury. The gels completely inhibited thrombosis at two hours post-surgery. At two weeks, control vessels were consistently obstructed, while treated vessels were completely patent and did not exhibit residual effects of injury. Future work will focus on the effects of the hydrogel material on vascular wound healing and on the effects of preventing thrombosis on intimal thickening.

\section{5}

BIPHASIC ANISOTROPIC MODEL OF CELL-MATRLX INTERACTIONS Victor $H$. Barocas and Roben T. Tranquillo

Department of Chemical Engineering and Materials Science, University of Minnesota

The interaction between fibroblasts and the surrounding extracellular matrix (ECM) is the driving force behind matrix restructuring in various physiological processes, in particular during the contraction phase of dermal wound healing. The fibers that compose the matrix are rearranged by the traction force exerted by fibroblasts, causing changes in local matrix concentration and orientarion. The changes in the ECM in turn affect the magnitude and orientation of the cell traction and migration.

A biphasic anisotropic model of the cell-ECM system has been developed based on previous monophasic model (Moon and Tranquillo, 1993) for cell-ECM interactions and an existing biphasic isotropic model (Dembo and Harlow, 1986) for cytomechanics. The and an existing biphasic isotropic model (Dembo and Harlow, 1986) for cytomechanics. The
model is biphasic in that the ECM is treated as distinct fibril and medium phases; it is model is biphasic in that the ECM is treated as distinct fibril and medium phases; it is
anisotropic in that the migration and the traction force of the cells are dependent on direction anisotropic in that the migration and the traction force of the cells are dependent on direction
and degree of orientation of the fibrils. The orientation distribution of the fibrils is and degree of orientation of the fibrils. The orientation distribution of the fribrils is and migration is determined by integrating over the disrribution.

The model bas been used to simulate in vitro fibroblast populated collagen microsphere (FPCM) assays. Using literature and experimental data, we predict the rate of compaction of the FPCM assay based on the traction parameter $\tau_{0}$, an intrinsic measure of the cell traction force. We compare observed reorientation of the collagen fibrils yith that predicted by the modei. Finally, we have used the modei to study a model in vivo wound. 
166

ADSORPTION OF ALBUMIN ONTO POLY (ETHER URETHANE)

J.S.Jeon 1 , R. Sperline 2 and S. Raghavan

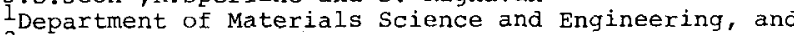

${ }^{2}$ Department of Chemistry, The University of Arizona, Tucson, AZ 85721

Adsorption of bovine serum albumin onto a Pellethane $2393 \mathrm{film}$ was investigated using an ATR FT-IR tech-

nique as a function of solution $\mathrm{pH}$ and albumin concentration. The extent of adsorption was calculated from the amide II peak area with the assumption of a steptype adsorption profile at the interface. At a $\mathrm{pH}$ of 7, the adsorption of albumin increased linearly with solution concentration and at a physiological concentration of $45 \mathrm{gm} / \mathrm{liter}$ the adsorption density was calculated to be $3.9 \mu \mathrm{g} / \mathrm{cm}^{2}$. The extent of adsorption was sensitive to solution $\mathrm{pH}$ and exhibited a maximum near neutral pH value.
169

EXTRINSIC INFRARED LABEIS FOR PROTEIN ADSORPTION

K.K. Chittur, S.A. Orr, J.M. Harris

University of Alabama in Huntsville, Huntsville, AL 35899 MN 55311-3648

The FT-IR/ATR technique for studying protein adsorption to solid surfaces allows us to get both quantitative and qualitative information. Using this technique we can correlate protein conformation (fibronectin) with cellular spreading and growth. We will present examples. A major problem with the technique is the difficulty of working with protein mixtures. While infrared alone can provide limited differentiation between the different plasma proteins, our studies have indicated that this is clearly not enough. Often the protein of interest is adsorbed to a surface that will have perhaps hundreds of other proteins. Can we "extract" the spectrum of the protein from the mixture? Once a spectrum is obtained, we can analyze the spectrum for changes in conformation. We have covalently linked infrared active groups (-CN) to albumin and gammaglobulin. This group can be clearly identified in a region free of interferences from water and other proteins. Our talk will highlight recent results from this study. We will present adsorption experiments with labeled albumin and IgG from single protein solutions and from mixtures. Experiments illustrating protein self-exchange on surfaces will also be presented.

170

KINETIC STUDY OF PROTEIN ADSORPTION BY FT-IR REFLECTION SPECTROSCOPY

P. Buscemi

Science Director, SCIMED Life Systems, 6655 Wedgewood Road, Maple Grove, MN 55311-3648

While the adsorption of plasma proteins, both singly and in multiple combinations has been well studied by internal transmission techniques, less work has been committed to the study of surfaces by reflection spectroscopy. This is interesting in that while probing beams may penetrate some distances through protein coatings providing information on several layers, it is the outermost surface that is in contact with tissues. In this work we attempt to monitor amounts and types of proteins adsorbed from both diluted and whole human plasma onto biodegradable surfaces. While the surfaces monitored have been aged in physiological buffers for periods of time up to several months, plasma solutions are exposed only for periods ranging from seconds to hours. This is relevant in that degrading surfaces may remove older layers thus exposing new surfaces to fresh plasma. Results are compared to previous works.

\section{8}

INTEGRATED OPTICAL WAVEGUIDE ATR SPECTROMETRY AND RESONANCE RAMAN SPECTROSCOPY OF ADSORBED CYTOCHROME C

D.S. Walker and W.M. Reichert

Department of Biomedical Engineering, Duke University, Durham, NC 27708-0281

Cyt c strongly adsorbs $514.5 \mathrm{~nm}$ light, has a well characterized resonance Raman spectrum, and its orientation at surfaces has been the subject of recent study [Pachence et al, Biophys. J., 58:379 laser i Fraalje et al, Biophys $J$. $57: 965$ (1990 horse heart cyt $c$ adsorbed to the surface of micror-thick siliconoxynitride adsorbed to the surface of micron-thick siliconoxynitride integrated optical waveguides (IOW), Measurements were performed in a custom built flow cell device using both TE (horizontal) and TM (within $4^{\circ}$ of vertical) polarizations of the incoupled laser light. IOW AlR measurements determined the dB/cm propagation loss attributed to the adsorbed protein film by imaging the guided mode intensity before and after protein adsorption. IOW resonance Raman spectra of the adsorbed protein monolayer were collected using a high throughput single grating spectrograph with a holographic filter for enhanced laser rejection. The waveguide Raman spectra are the first reported of a protein film adsorbed to a nonconducting surface. ATR and resonance Raman measurements were used to calculate adsorbed amount and infer orientational information.
171

PENETRATION OF CIPROFLOXACIN INTO $P$, aeruginosa BIOFILMS P.A. Suci, M.W. Mittleman, P. Yu, G.G. Geesey

Montana state University, Bozeman, Montana 59717

Ciprofloxacin is among the newer quinolone antimicrobial agents whose primary action is inhibition of DNA gyrase. Its penetration into the base of Pseudomonas aeruginosa biofilms was investigated using ATR/FT-IR methodology. The technique collects IR spectra originating from an interfacial region approximately one micron thick. Lateral colonization of the IRE surface was monitored by computing IR difference spectra in the frequency range from $1800 \mathrm{~cm}^{-1}$ to $850 \mathrm{~cm}^{-1}$. After a $50 \mathrm{~h}$ colonization period $100 \mu \mathrm{g} / \mathrm{ml}$ ciprofloxacin was introduced into the flow chamber for 21 minutes followed by reintroduction of culture media for 16 minutes. During this time interval spectra were acquired every 13.8s. Computed difference spectra revealed IR bands of the antibiotic. Rate of penetration and final concentration delivered to the base of the biofilm were estimated. spectral changes in both the antibiotic and the biofilms were observed from the interaction. Viability of biofilm bacteria with and without exposure to the antibiotic was determined. Biofilm morphology was characterized by standard microscopy and thin film sectioning. 
172

EFFECT OF CRYSTALLINITY OF CALCIUM PHOSPHATE COATINGS ON DISSOLUTION IN SALINE, PROTEN. FT-IR/ATR STUDY

Joo Ong (1), Linda Lucas (1), K.K. Chittur (2)

1) Biomedical Engineering, University of Alabama in Birmingham

2) Chemical Engineering, University of Alabama in Huntsville

Calcium-phosphate (Ca-P) coatings such as hydroxyapatite (HA) on dental and orthopaedic implants are primarily used to induce bony in-growth and to provide rapid fixation to bone. However, Ca-P coatings are not inert and their rate of dissolution is dependent on many factors such as chemical composition and structure. From our previous study in saline solution, the amorphous $\mathrm{Ca}-\mathrm{P}$ coatings $(0.6 \mu \mathrm{m}$ thick) were completely dissolved within 4 hours whereas the crystalline Ca-P coatings of similar thickness took a minimum of 9 weeks to dissolve. We will describe our recent study where we are using FTIR-ATR to evaluate the dissolution of crystalline and amorphous Ca-P coatings in saline, $\alpha-\mathrm{MEM}$, and $\alpha$-MEM with proteins.

\section{3}

IMAGING OF DNA BY STM AND AFM

R. J. Warmack, T. Thundat, and D. P. Allison

Oak Ridge National Laboratory, Oak Ridge, TN 37831-6123

Although imaging of biomolecules by scanning probe microscopy has been a challenge due to probe-sample interactions, examinations by both scanning tunneling and atomic force microscopes have now been made routine. Biomolecules do not ordinarily bind well to mounting substrates flat enough to permit topographic contrast. For STM, this resulted in only very rare and unstable sightings of the nonconducting, uncoated DNA molecules. We have successfully used spectroscopic (dI/dV) imaging to distinguish equally rare artifacts from poorly conducting adsorbates. Subsequently, a chemical treatment for gold substrates was developed that successfully immobilizes the DNA'. Now, large relaxed plasmids are seen at the expected density, so that artifacts are no longer a problem.

For AFM, large DNAs from kinetoplasts, lambda phages, and cosmids can be mounted upon mica and routinely imaged. Instrumental effects caused by cantilever buckling, tip adhesion, and tip size have been studied. Lateral forces have been calibrated to obtain micro-friction measurements. Attachment of proteins at specific sites would allow high resolution mapping. The current challenge for AFM of DNA is to image under physiological conditions so that protein interactions could be observed in vitro.

${ }^{1}$ D. P. Allison, L. A. Bottomley, T. Thundat, G. M. Brown, R. P. Woychik, J. J. Schrick, K. B. Jacobson, and R. J. Warmack, Proc. Not. Acad. Sci. 89, 10129-33 (1992)

Research sponsored by the Office of Health and Environmental Research, U.S. Department of Energy under contract DE-AC05-84OR21400 with Martin Marietta Energy Systems, Inc.

\section{4}

\section{VON WILLEBRAND FACTOR STUDIED BY AFM UNDER AMBIENT AND AQUEOUS CONDITIONS \\ C. Siedlecki, S.J. Eppell and R.E. Marchant,}

Case Western Reserve University, Cleveland, Ohio

Protein adsorption on biomaterials and subsequent surface-induced conformational changes are determined by interactions which depend the properties of the material surface and the plasma protein. We report on a properties of the material surface and the plasma protein. We report on a
molecular-level study by atomic force microscopy (AFM) of von Willebrand Factor (vWF) on hydrophilic and hydrophobic surfaces. In our early experiments on hydrated vWF deposited on mica, the protein molecules were casily swept across the surface by the lateral force applied by the probe tip. Removal of interfacial water by drying increased protein-surface adhesion and permitted molecular level imaging in air, in which the distinctive globular domains of vWf were resolved. Surface features down to $20-40$ nanometers in the $x-y$ plane of the protein were resolved, compared with nanometer resolution in the height direction. In recent experiments, we have successfully imaged vWF under aqueous buffer conditions, by using a surface modified by an octadecyltriclorosilane (OTS) selfassembled monolayer on glass. The hydrophobic OTS monolayer increased the protein-surface adhesion permitting the vWF to be imaged. A sub-monolayer protein-surface adhesion permitting the vWF to be imaged. A sub-monolayer globular domains. Analysis of the structural information obtained under aqueous conditions showed a close similarity with our previous data on vWF obtained in air. Our results demonstrate that globular proteins can be imaged by AFM under aqueous as well as ambient conditions. Larger scale images can be used to determine organization and coverage of a surface, while high resolution images can be used to measure molecular dimensions of the adsorbed protein.
175

TOTAL INTERNAL REFLECTION FLUORESCENCE MICROSCOPY (TIRFM): TOPOGRAPHICAL MAPPING OF RELATIVE CELL/SUBSTRATE SEPARATION DISTANCES

J.L. Yarbrougb, J.S. Burmeister, G. A. Truskey, and W. M. Reichert (Department of Biomedical Engipeering, Duke University, Durham, NC 27706)

Total internal reflection fluorescence microscopy (TIRFM) is a technique developed to excite fluorescent regions of cells in closest apposition to the substrate surface. Using the theory of Truskey et al., J. Cell Sci. 103: 491-499, 1992, TIRFM permits relative quantitation of the cell-substrate separation distances. The contact area between cells and substrate is an important parameter when measuring the strength of adhesion of cells to surfaces. Phase contrast and TIRFM images of bovine aortic endothelial cells (BAEC) plated onto glass microscope slides were collected. Calculated cell/substrate separations from theoretical fits to the TLRFM image brightness were used to construct a topographical map of cell separations over the ventral surface of the cell membrane The results indicate that ITRFM can provide quantitative information about changes in the cell/surface contact area.

176

ROLE OF ADSORBED PROTEINS IN ANIMAL CELL ADHESION TO FOREIGN SURFACES

Thomas A. Horbett

Chemical Engineering and Bioengineering, University of Washington, BF-10, Seattle, WA 98195

Most cells in animals are adherent throughout their lives, e.g., fibroblasts and myoblasts. Even those that are not normally adherent such as the platelets and white cells in blood must undergo an adhesion step to perform their ultimate biological function. Many of the adhesion reactions of animal cells are mediated by membrane bound receptor proteins that bind reactions of animal cells are mediated by membrane bound receptor proteins that bind
specifically to specialized adhesion proteins such as fibronectin, vitronectin, and fibrinogen. specifically to specialized adhesion proteins such as fibronectin, vitronectin, and fibrinogen.
Therefore, the focus of this presentation will be on the adsorption behavior of the adhesion Therefore, the focus of this presentation will be on the adsorption behavior of the adhesion proteins, the properties of the receptor proteins, and the interaction of these elements in the adhesion of cells to surfaces containing adsorbed adhesion proteins. The presentation will largely concern cell adhesion to foreign surfaces.
177

CHARACTERIZATION AND INITIAL BIOCOMPATIBILITY STUDIES OF NOVEL TINbZ AND ZNNb MATERIALS

S.M. Slack, Y. Yang, G. Lewis, J. Davidson, K. Daigle, and V.T. Turitto, Dept. of Biomedical Engineering, Memphis State University, and Smith \& Nephew Richards Inc., Memphis, TN

Initial studies of the potential biocompatibility of two novel Ti13Nb13Zr (Ti) and oxidized $\mathrm{Zr} 2.5 \mathrm{Nb}(\mathrm{Zr})$ materials were conducted. Using ${ }^{125} \mathrm{I}$-fibrinogen, the amount of fibrinogen adsorbed to these materials from both pure solutions and human plasma was determined; in addition, the ability of these materials to support activation of the intrinsic coagulation pathway was measured using chromogenic assays for the presence of Factor $X a$ and kallikrein, one of the initial products of contact activation. The amount of fibrinogen adsorted from pure solutions to both materials ranged from 20 to $300 \mathrm{ng} / \mathrm{cm}^{2}$ as the fibrinogen concentration varied from 0.001 to $0.3 \mathrm{mg} / \mathrm{ml}$. From plasma, fibrinogen adsorption increased with increasing plasma concentration on both materials and was greater on $\operatorname{Zr}\left(50 \mathrm{ng} / \mathrm{cm}^{2}\right)$ than $\mathrm{Ti}\left(25 \mathrm{ng} / \mathrm{cm}^{2}\right)$ at a plasma concentration of $10 \%$. The amount of functional Factor $X a$ adsorbed to $T i$ and $Z$ determined from a chromogenic assay using the Spectrozyme substrate, was similar to that measured on the negative controls (silicone ruzber) and was several times loss than that on less than that measured using glass. Finally, kallikrein generation by $\mathrm{Ti}$ and $\mathrm{Z}$ surfaces previously exposed to human plasma was approximately half of that observed wh materials are poor activators of the intrinsic coagulation cascade and that they adsort fibrinogen in amounts sils as silicone rubber, which are currently used in clinical settings. Based on these results, the $\mathrm{Ti}$ and $\mathrm{Zr}$ materials appear to be promising candidates for use in bloodcontacting devices and merit further investigation. 
178

INTEGRIN/CYTOSKELETON INTERACTIONS IN MIGRATING FIBROBLASTS ARE DYNAMIC, ASYMMETRIC, AND REGULATED Schmidt, C.E. ${ }^{*}$, A.F. Horwitz ${ }^{\dagger}$, D.A. Lauffenburger ${ }^{*},+$

Depts. of ${ }^{*}$ Chemical Engineering and ${ }^{\dagger}$ Cell \& Structural Biology, Univ. of Illinois at Urbana-Champaign

Integrin adhesion receptors are key components involved in cell migration over extracellular matrix proteins, transmitting intracellular motile force into traction on the surroundings. The mechanism by which integrin interacts with cytoskeletal elements, permitting force transmission, is presently not understood. We have applied nanovideomicroscopy and laser optical trapping methods to explore integrin/cytoskeleton interactions by following the dynamics of small particles coated with anti-integrin antibodies following attachment to the dorsal surface of fibroblasts. We have further employed site-directed mutants of the integrin $\beta 1$ chain cytoplasmic tail to investigate the role of this domain in regulating these interactions. We find that integrin/cytoskeleton association is reversible, that it requires some part of the $\beta 1$ chain cytoplasmic domain, that it is affected by mutations mimicking or preventing phosphorylation events on certain serine and tyrosine residues. Significantly, we also find that these interactions are asymmetric across the dimension of a migrating cell with stronger interactions in the front than at the rear. These findings help develop an improved picture of the role of integrin in cell migration, with implications for control of cell migration by extracellular matrix molecules.

179

MODEL STUDIES OF RECEPTOR-MEDIATED CELL ADHESION USING RAT BASOPHILIC LEUKEMIA CELLS.

D.A. Hammer, L. A. Tempelman and L. Chu

School of Chemical Engineering, Comell University, Ithaca, NY 14853.

We have developed a model system to study how cell and substrate chemistry affect receptor-mediated cell adhesion. In a flow chamber, we measured the adhesion of rat basophilic leukemia (RBL) cells to polyacrylamide gels covalently derivatized with the ligand dinitrophenol (DNP). The RBL cells possess $\mathrm{Fc}_{\mathcal{E}}$ receptors, which are filled with different numbers of $\mathrm{IgE}$ molecules that recognize DNP. In this system, we control the number of DNP binding sires that recognize DNP. In this system, we control the number of and the DNP surface density, as well as fluid shear rate. We on the cell surface and the DNP surface density, as well as fluid shear rate. We
quantify adhesion by measuring the surface density of adherent cells on the gel quantify adhesion by measuring the surface density of adherent cells on the gel
surface as well as the percentage of cells which have entered the chamber which surface as well as the percenteg
ultimately adhere to the gel.

There is a narrow range of ligand density, IgE surface density, and shear
Thel. rates for which the percent adhesion can be modulated. By prebinding $\operatorname{IgE}$ to the gel surface, and using the slower kinetic recognition between IgE and $\mathrm{FCE}_{\mathrm{E}}$ receptor, we show that low reaction rates and high shear rates lead to similar spatial patterns and extents of adhesion, consistent with a single dimensionless scale of reaction rate to shear rate which controls attachment. Adhesion in all cases exhibits a kinetic variability in which cells can adhere anywhere up to a thousand cell diameters from the start of the DNP portion of the gel.

We complement these studies with centrifugation assay measurements of adhesion strength of IgE-sensitized RBL cells to DNP-coated gels after short $(\leq$ 20 min) incubation times. When DNP and IgE densities are low, single IgE-DNP binding events mediate adhesion; centrifugation fields of $100-200 \mathrm{~g}$ reverse this adhesion and give an estimate of $2-4$ microdynes as the strength of the DNP-IgE$\mathrm{Fc}_{\mathcal{E}}$ receptor bond. Under most DNP and IgE densities, adhesion can be reversed with forces $\leq 13$ microdynes, suggesting very few bonds mediate adhesion.

180

CULTURE OF HUMAN BONE MARROW PROGENITOR CELLS IN AN ARTIFICIAL CAPILLARY PERFUSION CULTURE DEVICE William Kidwell, Cellco, Inc., Germantown, Maryland.

Mononuclear cells derived from human bone marrow have been cultured in an artificial capillary culture device. The mononuclear cells secrete an autocrine factor that inhibits the growth of the producer cells. In the culture device, cells are plated on the outer periphery of the capillaries where the cells grow and receive their nutrients and oxygen from recirculating medium flowing through the lumen of the artificial capillaries. When one uses capillaries having ultrafiltration properties, the inhibitor accumulates around the cells and blocks cell growth. However, when the capillary wall "pore" is large, as is the case with microporous hollow fibers, the inhibitor diffuses away from the cells and is diluted to noneffective concentrations, permitting cell growth. In addition to pore size, bone marrow cell growth is affected by co-cultivation with stroma, by the media perfusion rate and by coating the fibers to enhance cell attachment. Under optimum conditions, as much as 10,000 fold increases in cell number have occurred. The culture method may be useful for the production of large numbers of bone marrow cells for regenerating immune function following myeloablative chemotherapy.
181

BIOFOULING OF MEMBRANES

Kimberly Ward Anderson and Sue H. Strange

Department of Chemical Engineering, University of Kentucky, Lexington, KY 40506

Biofouling, or the adsorption and growth of bacteria on membranes is a topic of great industrial concern. Bacteria which adhere to membranes grow and reproduce and may eventually form a biofilm, a combination of cells and extracellular polymeric substances that hold the matrix of cells together. The development of the biofilm results in decreased membrane flux. To this date, the precise mechanisms by which bacteria adhere to surfaces is unknown. In this investigation, the parallel plate flow chamber is used to quantify the adhesion of bacterial cells to membranes. A thin layer of the desired membrane is placed onto the glass slide of the chamber. A dilute suspension of bacterial cells is placed in the chamber and individual cells are allowed to settle onto the membrane for various times ranging from 5 minutes to 2 hours. The membrane with adherent cells is then perfused with culture medium at various flow rates to determine the number of cells that detach as a function of shear stress. Two strains of bacterial cells that were studied include Escherichia coli and Staphylococcus epidermidis. The effects of surface properties of the bacteria, membrane surface properties and growth phase of the bacterial cells on the adhesion properties will be discussed.

182

EFFECT OF PEPTIDE CONFORMATION ON THE STRENGTH OF ADHESION OF ENDOTHELIAL CELLS TO IMMOBILIZED-RGD SURFACES Yao Xiao and George A. Truskey, Center for Biochemical Engineering and Department of Biomedical Engineering, Duke University, Durham, NC 27708-0277

The objective of this study was to determine the effect of ligand conformation on the strength of endothelial cell adhesion. Two different conformational forms of the fibronectin cell binding domain peptide, Arg-Gly-Asp (RGD), were used, a higher affinity cyclic RGD and a linear RGD. The peptides were covalently immobilized on non-adhesive glycophase glass stides. Bovine aortic endothelial cells were dissociated from tissue culture flasks with EGTA. Cells were incubated with the surfaces in serum-free Dulbecco's modified Eagle's medium containing $10 \%$ bovine serum albumin for $15 \mathrm{~min}$ followed by exposure to laminar flow for 5 seconds in a variable height flow chamber. Shear stress ranged from $10-80 \mathrm{dyne} / \mathrm{cm}^{2}$. At a ligand density of about $5 \mathrm{fmol} / \mathrm{cm}^{2}\left(3 \times 10^{9}\right.$ molecules $\left./ \mathrm{cm}^{2}\right)$, the critical shear stress at which $50 \%$ of the cells were detached was $50 \%$ higher on surface containing cyclic RGD peptide than the linear RGD peptide. This difference corresponded to approximately 13 fold higher affinity of cyclic RGD to the receptor than that of linear one. At higher ligand densities, no effect of peptide conformation was observed, presumably because a sufficient number of bonds were formed to tesist the hydrodynamic forces.

183

THE EFFECT OF BIOMATERIAL SURFACE AND SHEAR ON THROMBIN GENERATION AND PLATELET ACTIVATION IN WHOLE BLOOD

J. Jato, P. Kao and R. Beissinger, Illinois Inst. Tech., M. Telfer and C. deLannoy, Michael Reese Medical Center, Chicago, IL. and V. Shankey and J. Fareed, Loyola University Medical Center, Maywood, IL.

Our recent work has suggested a role for the local action of thrombin at a biomaterial surface (fluorinated ethylene propylene copolymer) based on the platelet deposition results found during simple shear flow of whole blood. The objective of this study was to determine if thrombin was generated in the bulk fluid and if platelets were activated as a function of shearing conditions. Rat whole blood was defibrinated (and therefore anticoagulated) invivo with Ancrod, the purified coagulative fraction of Malayan pit viper venom. Whole blood samples were collected and sheared in a cone-and-plate viscometer to shear rates up to about $5000 \mathrm{~s}^{-1}$, which corresponded to shear stresses of $150 \mathrm{dyne}^{\prime} \mathrm{cm}^{2}$. Although thrombin activity was found, it was shown to not be a function of the shear imposed on the blood samples in the bulk fluid. These preliminary results suggested, based on the value known for maximum thrombin potential in human whole blood $(17.5 \mathrm{NIH}$ units $/ \mathrm{ml})$ compared to that found in this study for rats $(6.5 \mathrm{NIH}$ units/ml), that the potential for additional thrombin generation had not yet been saturated. However, addition of tissue thromboplastin to the Ancrod-defibrinated rat whole blood samples did not result in any further thrombin generation, suggesting possibly that thrombin was being generated due to the method of blood removal from the rat. Currently, we are evaluating if human whole blood, which is defibrinated in-vitro using Ancrod, results in shear-induced thrombin generation and platelet activation in the bulk fluid. Activation of platelets is being measured using flow cytometric analysis of CD-62 monoclonal antibody (GMP-140) expression on CD-61 monoclonal antibody (GP IIb/IIIa) positive platelets. 
184

LYMPHOCYTE ADHESION TO ENDOTHELIAL CELLS UNDER FLOW CONDITTONS Larry V. McIntire ${ }^{\dagger}$, David A. Jones ${ }^{\dagger}$, Louis J, Picker*, and C. Wayne Smith ${ }^{\ddagger}$

Cox Laboratory for Biomedical Engineering, Rice University ${ }^{\dagger}$ Houston, TX, 77251-1892. Baylor College of Medicine ${ }^{\ddagger}$, Houston, TX; University of Texas Southwestern Medical Center*, Dallas, TX

The process of tymphocyte extravasation is believed to consist of specific receptormediated adhesion of lymphocytes to endothelial cells followed by activation of the lymphocytes and transmigration into the extravascular space. We have developed an in vitro model system for quantitative study of these processes. Appropriate flow conditions are produced using a parallel-plate flow chamber in which isolated peripheral blood T-cells are passed over a monotayer of human umbilical vein endothelial cells (HIVEC) or $T$-cells are pell lines. cell lines. Adhesion is quantified using phase-contrast video microscopy coupled to digital adherent, and diapedesed lymphocytes as well as measure rolling velocities.

Or initial studies focus on the interactions between lymphocytes and endothelial cells mediated by receptors of the selectin and integrin families. These results suggest that as previously seen with neutrophivendothelial cell interactions, selectins are most important in arresting flowing lymphocytes from the free stream and mediate a rolling interaction. Integrins, on the other hand, appear unable to arrest freely flowing lymphocytes, but can mediate shear-resistant firm adhesion once initial selectin-mediated interactions have slowed the lymphocytes. These findings are based on experiments using tissue culture conditions we have developed to induce expression of particular endothelial cell adhesion receprors, using cell lines transfected with particular adhesion receptors, and using fluorescence-activated cell sorting to isolate particular $T$-cell subsets.

185

LEUKOCYTE MIGRATION IN A NOVEL DISPERSED CELL FIBRIN GEL ASSAY Prabhas V. Moghe and Robert T. Tranquillo

Department of Chemical Engineering and Materials Science, University of Minnesota

In yizro assays of cell migration in response to chemotactic factors (CF) are of crucial importance to clinical diagnoses and scientific investigations. Meaningful parameters of population-level cell response in these assays can be measured in terms of phenomenological coefficients, $\mu$ and $\chi$, the random motility and chemotaxis coefficients. of a constitutive cell flux equation characterising intrinsic cell movement in response to $C F$. The traditional under-agarose and Boyden filter assays, while popularly used, involve a concentrated cell source. These high cell densities invalidate assumptions of an isolated cell response to a well-defined CF field implicit to the theory behind definition of $\mu$ and $x$.

In response to the obvious need for a simple assay that removes these high cell density-associated complications, we have developed a novel dispersed cell assay. It involves
starting with human blood cells dispersed uniformly at a suitably low density throughout a starting with human blood cells dispersed uniformly at a suitably low density throughout a
reconstituted fibrin or collagen gel followed by presentation of a CF diffusion gradient. reconstituted fibrin or collagen gel followed by presentation of a CF diffusion gradient. Automated image analysis and video microscopy are used for measurement of depart
cell density from the intial uniformity due to chemotaxis to yield values of $\mu$ and $\chi$

The focus of this paper is the quantitation of cell migration by direct observation
sity from the intial uniformity due to chemotaxis to yield values of $\mu$ and methods in the dispersed cell assay using time-lapse video microscopy and cell tracking. This is the first quantitative characterization of neutrophil chemotaxis for a large cell population in three-dimensional gels. Intermediate magnification two-dimensional projected tracking and high magnification three-dimensional tracking algorithms are used to generate cell tracks which are then processed to yield population level $\mu$ and $\chi$, as well as single cell movement indices, cell speed and persistence time, as a function of CF concentration and gradient stecpnesses. These porsistence time, as a function or $C F$ concentration and gradient stecpnesses. These powerful techniques should be valuable in studying the extent and the geis used for tissue engineering.

186

EFFECT OF CELL AGE ON TISSUE FACTOR ACTIVITY IN MACROPHAGE CELL CULTURES SUBJECTED TO FLUID SHEAR Lee J. Carmack, Steven M. Peckham, Yale Nemerson*, and Vincent $T$. Turitto (Department of Biomedical Engineering, Memphis State University, Memphis, TN 38152, and *Mount Sinai Medical Center, New York, NY 10029)

We have investigated the effects of fluid shear on the coagulative behavior of cultured macrophages derived from peripheral blood monocytes. Using a parallel plate perfusion chamber cells cultured 7 to 14 days on Thermanox slides were exposed to wall shear rates of 6 to $31 \mathrm{~s}^{-1}$ for up to 2 hours in $0.01 \mathrm{M}$ Hepes buffer containing $100 \mathrm{nM}$ factor $X$ (FX) and $10 \mathrm{nM}$ factor VIIa (FVIIa). The conversion of FX to FXa by tissue factor was determined by means of a chromogenic assay used to measure the concentration of FXa exiting the chamber. Monocytes can be stimulated in vitro by bacterial endotoxin and calcium ionophore A23187 on the day of collection to convert blood coagulation factor $X$ to FXa. Our preliminary studies with mature macrophages show that shear of cells with a solution of calcium ionophore A23187 $(10 \mu \mathrm{g} / \mathrm{ml}$ ) enhances the conversion of blood coagulation factor $\mathrm{X}$ to factor $\mathrm{Xa}$ on cultures subjected to a shear rate of $31 \mathrm{~s}^{-1}$. However, exposure to endotoxin ( $1 \mathrm{\mu g} / \mathrm{ml}$ for 4 hours) had little or no effect on this procoagulant activity in these older cell cultures.
187

EFFECT OF SHEAR FORCES ON ICAM-1 EXPRESSION IN HUVECS. R. Sampath, L.V. McIntire, S.G. Eskin ${ }^{1}$, and C.W. Smith ${ }^{2}$ Cox Laboratory for Biomedical Engineering, Rice University, Houston, TX $\mathbf{1}_{\text {Cell Biology Division, Texas Biotechnology Corporation, Houston, TX }}$ ${ }^{2}$ Dept. Of Pediatrics, Baylor College of Medicine, Houston, TX .

Extensive monocyte recruitment is an early phenomenon associated with the development of atherosclerotic lesions, suggesting an active role for the adhesion molecules, ICAM-1 and VCAM-1 expressed by endothelial cells, which bind monocytes. Shear stress has previously been shown to selectively modify protein secretion and message for RNA
(mRNA) levels of cerain molecules like tissue plasminogen activator (tPA) known to be (mRNA) levels of certain molecules like tissue plasminogen activator (LPA) known
associated plaque formation and fibrin deposition. In this study we describe the associated plaque formation and fibrin deposition. In this study we describe the
contribution of hemodynamic shear forces in regulating the monocyte adhesion process in contribution of hemodynamic shear forces in regulating the monocyte adhesion process
the presence or absence of other stimuli. Human umbilical vein endothelial cells (HUVECs) were subjected to arterial levels of shear $\left(\approx 25 \mathrm{dynes} / \mathrm{cm}^{2}\right)$ and analyzed either for surface expression of ICAM-1 using flow cytometry or for shear induced changes in ICAM-1 mRNA levels by Northern blot technique. Results were compared to basal levels obtained from unstimulated cells, and positive controls from lipopolysaccharide (LPS) treated cells. Preliminary results from the fluorescence analysis show no effect of prolonged shear stress on ICAM-1 expression. However, a transient increase in the expression of ICAM-1 was seen after 12 hours of exposure to arterial levels of shear that returns to basal levels within 24 hours, quite different from the well described time dependent response of ICAM-1 expression to LPS. Similar studies are underway to dependent response of ICAM-1 expression to LPS. Similar studies are underway to function of time and the effect of stress magnitude (venous vs arterial levels) on the time history of ICAM-I expression.

188

FLUORESCENCE FLOW CYTOMETRIC ANALYSIS AND MATHEMATICAL MODELING OF THE KINETICS OF NEUTROPHIL AGGREGATION. S.I. Simon, T.R. Lucero ${ }^{+}$, L.A. Sklar ${ }^{+}$, and, M. Dembo*. Dept. Pediatrics. Baylor College of Medicine, Houston TX 77030. ${ }^{+}$Dept. of Pathology. Univ. of New Mexico, Albuq. NM. 87131. 'Theoretical Biol. T-10, Los Alamos Natl Labs, Los Alamos NM. 87545.

Human neutrophil suspensions shear mixed in a test tube exhibit reversible aggregation in response to formyl peptide stimulation. Singlet recruitment to form larger particles is most rapid over the first minute, at which time formed aggregates remain attached for several minutes until disaggregation rapidly ensues. Mathematical modeling of this phenomenon enables the prediction of particle kinetics in terms of unambiguous rate constants. The rate constants provide insight into several aspects of the process including the adhesion efficiency of cell encounters and the dependence on cell concentration. Recently, we have elucidated two adhesion receptors, B2-integrin and L-selectin, as molecular requirements for aggregation. In these studies we employed fluorescence flow cytometry to identify two populations of cells, one labeled with a red fluorescent dye the other labeled green. In this way it was possible to measure the aggregation kinetics of red-red, green-green, and red-green cell populations. By treating individual populations with lipopolysacharide endotoxin which alters the expression of adhesion molecules, we explored the change in adhesive avidity. We formulated a model to predict the aggregation kinetics between mixed cell populations. The rates of reaction are expressed in terms of three association rate constants $\left(k_{a R R}, k_{O G G_{T}}\right.$ and $\left.k_{\Delta R G_{G}}\right)$, and three dissociation rate constants $\left(k_{d R R}, k_{d C G}\right.$, and $k_{d R O}$ ). Differential equations were set up to describe 49 reversible reactions between 29 possible aggregate species from singlet to quartet combinations of red and green cells. The model was successful at fitting particle kinetics between control and endotoxin treated cell populations. 
189

SENSTIVTYY AND RESOLVABIUTY OF PHASED ARRAY SPECTROSCOPY K.A. Kang and $B$. Chance

Dept. of Biophysics, Univ. of Pennsyivania, Philadelphia, PA 19104

NIR Phase Modulated Spectroscopy (PMS) characlerizes a system by measuring the mean transport delay (between a source and a detector) of a sinusoidally modulated light wave in terms of the phase delay of the wave. In a homogeneous media, the wave propagation of PMS is concentric from the source position. Therefore, by correlating the information of the phase and the $A C$ light intensity, the absorption and the scattering of a system can be characterized. Two sources with the same AC amplitude and different initial phases, 0 and $\pi$ radians, may create a phase jump of $\pi$ radians and a null intensity in the center plane of the two sources in a homogeneous system. In the presence of an absorber, the in and anti-intensity compensation loses its balance and the null plane becomes distorted to the direction of the absorber.

In this talk, basic principles of in- and anti-phase sourced PMS (Phased array) are explained. The detectability of a single heterogeneity by using phased array is discussed. and the resolvability of this instrument for multiple absorbers is correlated with system and instrumental parameters.

190

DETECTION OF TUMORS WITH BACKSCATTEREO LIGHT

I.J. Bigio, J. Boyer, T. Johnson, J. R. Mourant, and T. Shimada. Los Alamos National Laboratory, CLS-5, MS-E543, Los Alamos, NM 87545 D.J. Costello, Lovelace Scientific Resources

R Ley, and D. Conn, Lovelace Medical Foundation

R. Glickman, D. Gritz, K. Storey-Held, Dep. of Ophth., UTHSCSA.

Los Alamos National Laboratory has been developing a versatile instrument, referred to as the Optical Biopsy System (or OBS) for noninvasive, real-time diagnosis of tissue pathologies. The OBS employs a small fiber-optic probe that is amenable to use with any endoscope or catheter, or to direct surface examination (e.g.. as in skin cancer, superticial lesions or cervical cancer). The probe is designed to be used in optical contact with the tissue under examination and has separate illumination and collection fibers. Backscattered light, which carries information about the elastic scattering properties of the tissue's cellular structure, (and fluorescence if desired) is examined over the wavelength range 250-800 nm. Differences in the measured spectrum indicate differences in tissue pathology as we have demonstrated in several in vitro measurements. We have also conducted in vivo clinical testing in various organ areas. For example, we have shown that in vivo measurements on human conjunctiva reliably distinguish lesions from normal conjunctiva. Recently. we have also performed in vivo measurements on skin cancers in animal models. a wider range of superficial leslons of the eye, and bladder cancer in humans. Data from in vivo experiments on both animals and humans will be presented.

191

UTILITY OF THE BEER-LAMBERT LAW IN NIR SPECTROSCOPY

Liszka HA and Ultman JS, Penn State University.

Hall $M$, Comfort BJ and Piantadosi $C A$,

Duke University Medical Center.

One strategy for employing near infrared spectroscopy (NIR) is to irradiate a tissue region with a laser source and to detect scattered photons at an angle of $30^{\circ}$ relative to the incident light beam. A relative calibration of this method assumes that the Beer-Lambert Law is valid for the 3-dimensional transport of photons. This calibration might be fully quantified by utilizing the pathlength of photons originating from a pulsed laser source. To determine if this is feasible, the intensity-time distribution of photons from a pulsed $775 \mathrm{~nm}$ laser diode were detected in $10^{\circ}$ increments around the perimeter of a twelve-inch diameter cylindrical container filled with FC-43 fluorocarbon emulsion (The Green Cross Corp). While the integrated photon intensity was greatest at a detection angle of $180^{\circ}$ and decreased to a minimum at $\pm 90^{\circ}$, the mean time-of-flight of photons was smallest at $180^{\circ}$ and increased to a maximum at $\pm 90^{\circ}$. This behavior could be explained by a Monte Carlo simulation that incorporated a high degree of forward scattering. Photon absorption is now being added to the simulation so that calibration by the Beer-Lambert Law can be tested.
192 NEAR IR FOURIER SPACE GATE AND ABSORPTION SHADOWGRAM IMAGES
THROUGH RANDOM MEDIA

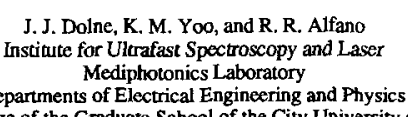

Departments of Electrical Engineering and Physics
The City College of the Graduate School of the City University of New York New York , NY, 10031

Shadowgram images of objects hidden behind random intralipid solutions media were studied using a Fourier spatial filter system using visible and NIR lights. Three laser diodes operating at $670 \mathrm{~nm}, 830 \mathrm{~nm}$, and $1300 \mathrm{~nm}$ were used to illuminate the samples. These three wavelengths fall in the therapeutic window where light can penetrate deep into the human body and reach intermal structures. Clear images are obtained by eliminating the diffuse light and letting only the ballistic/ sriake light through. This is achieved by placing a spatial fitter in the Fourier plane that cuts spatial frequencies higher than its cut-off frequency. Some of the diffuse light thas comes out paraxial to the ballistic/ snake light may be eliminated by the absorption of the random medium. The decrease in scattering and the increase in absorption of these media at longer wavelengths were found to significantly improve the image quality at longer wavelengths. The image contrast at $1300 \mathrm{~nm}$ was significantly better at $1300 \mathrm{~nm}$ than $830 \mathrm{~nm}$ and $670 \mathrm{~nm}$.

We thank Mediscience Technology Corp, and NSF for partial support of this research

193

A MEASUREMENT OF ABSOLUTE ABSORPTION SPECTRA IN A MULTIPLY SCATTERING MEDIUM

Joshua B. Fishkin, Peter T. C. So, Sergio Fantini, Maria Angela Franceschini, and Enrico Gratton.

University of Ilinois at Urbana-Champaign, Department of Physics, Laboratory for Fluoreacence Dynamica, Urbana, IL 61801.

By combining visible intensity modulated illumination with frequency domain detection methods, we have used a diffusion model to determine the absolute absorption and scattering coefficients of a strongly scattering medium. This medium consists of a uniform mixture of horse hemoglobin, Liposyn ${ }^{\star}$ III $20 \%$ (an intravenous fat emulsion), and water. The spectra were obtained using a rhodamine 6G dye laser that was pumped by a mode-locked Nd:Yag laser (frequency doubled to $532.8 \mathrm{~nm}$ ). The wavelength range covered was $560 \mathrm{~nm}$ to $620 \mathrm{~nm}$, in $5 \mathrm{~nm}$ increments. The absorption spectra obtained of horge hemoglobin in this multiply scatterering medium were compared with horse hemoglobin in a sodium phosphate buffer. The values of absorption of the relatively nonscattering medium were obtained by applying the Beer-Lambert equation to steady-state measurements. The absorption apectra obtained for the multiply scattering media (using the diffusion model) and the relatively non-scattering media (using the Beer-Lambert equation) were similax. These results illustrate the power of time resolved methods in obtaining absolute absorption spectra from multiply scattering media, and they point toward the possibility of using frequency domain methods for the spectroscopy of thick tissues. This work was performed at the Laboratory for Fluoregcence Dynamics at the University of Illinois at Urbana-Champaign (UIUC), which is supported by the Nation Institutes of Health (NIH), grant RR03155 and by UIUC. This work is also partially supported by grant CA57032 from the NIH. 
194

OPTICAL INDICATOR DILUTION MEASUREMENT OF LUNG PERMEABILITY-SURFACE AREA (PS) IS EQUIVALENT TO RADIOISOTOPE PS AFTER ALLOXAN INJURY IN THE DOG.

L.E. Olson, S. DeMarino, A. Pou, R.L. Galloway and T.R. Harris.

We developed a multiple indicator dilution (MID) technique to measure lung PS using optically absorbing tracers instead of radioisotopes. We obtained optical MID studies by measuring the change in optical density of blood at selected wavelengths. The optical tracers we used were sulfhemoglobinated erythrocytes (S) measured at $620 \mathrm{~nm}$, indocyanine green-bound albumin (I) measured at $820 \mathrm{~nm}$, and 1,2-propanediol (P) measured at $9.55 \mu \mathrm{m}$. Previously, we reported that PS values using both optical and radioisotope MID methods increased after alloxan infusion in the anesthetized, cannulated dog (FASEB J. 7(3):A2924, 1993). We discovered an overlap signal of in vivo $\mathbf{P}$ injections onto $S$ and measurement channels and an in vitro transfer function between I (or S) measurement and $\mathrm{P}$. Correction of optical MID studies for these overlaps gave optical PS values at baseline and after alloxan injury which, when regressed against radioisotope PS values, gave an $\mathrm{r}^{2}$ of 0.93 and a slope of 0.99 . We conclude that optical MID is an acceptable alternative to radioisotope MID. This work was supported by HL39155.

195

OPTICAL FIBERS AND FLUORESCENCE SPECTROSCOPY USED IN THE BACTERIA IDENTIFICATION OF OTITIS MEDIA Lou Reinisch

Department of Otolaryngology, Vanderbilt University Medical Center,

Nashville, Tennessee.

The management of acute otitis media in children continues to be hampered by two fundamental problems: First, the prevalence of bactericidal resistant species has been increasing (frequent misdiagnosi leads only to enhancement of the growth conditions for the resistan species). Second, the identification of resistant species requires a painful invasive procedure--tympanocentesis. To develop a rapid and non invasive identification technique, we have investigated the fluorescence profiles of four common pathogens: $P$. aeruginosa, $S$. aureus, $B$. catarrhalis and $H$. influenzae. The steady state auto fluorescence spectra of bacteria are measured as a function of the incident light from 200 to 700 $\mathrm{nm}$. The spectra for each bacterium are combined into a fluorescence profile or fluorescence finger print. Each bacterium produces a unique in vitro fluorescence profile when measured in a saline suspension. The profiles are reproducible, and independent of the bacteria growth stage. Suspensions of a bacterial strain, where the identification is not known can be identified using a linear least squares fitting algorithm of the known fluorescence profiles. Thus, we have established the precept for a fluorescence based bacterial diagnosis technique to be used in otitis media.

196

DETECTION OF FLUORESCENT LIFETIMES FOR NON-INVASIVE CHARACTERIZATION OF TISSUE METABOLIC STATE

C.I. Burch and E.M. Sevick

Local tissue environment (i.e., pH, glucose, NADPH levels and oxygen tension) may be non-invasively probedin vivo with newly designed near-infrared fluorophore dyes and time-dependent measurements of photon migration. Time-dependent measurements consist of injecting a delta pulse of light into the tissue or scattering media of interest and recording the time dependence of the re-emergent light. The re-emergent light is peaked, yet broadened due to the optical properties of the tissue and the fluorescence lifetime of the fluorophore. From finite element simulations which utilize the diffusion equations describing light transport in tissue light transport in deconvolved frof tissue scattering properties. The development fron the noninfrared chact is infrared light is proposed. Supported by The (BCS, NYI award).
197

FLUORESCENCE SPECTROSCOPIC DIAGNOSIS METHOD FOR GYNECOLOGICAL CANCER AND NORMAL TISSUES

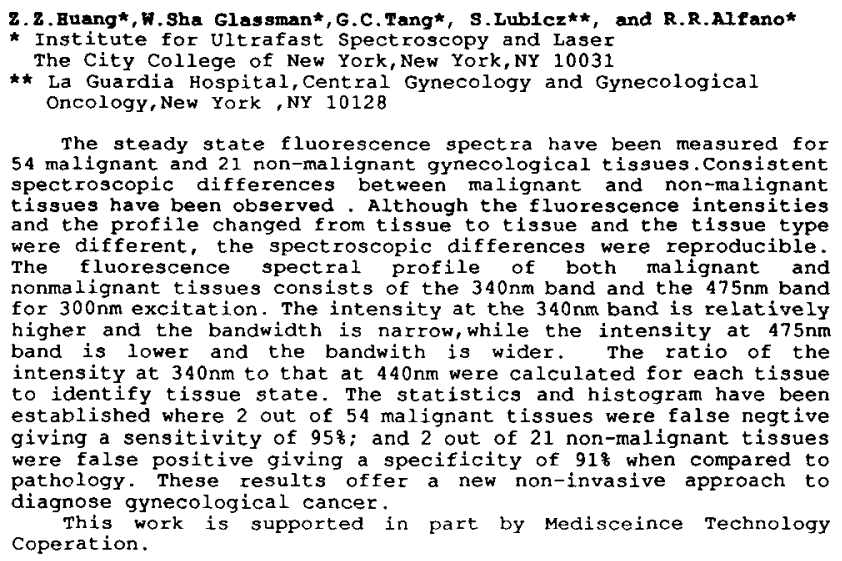

198

RAMAN SPECTROSCOPY FOR DIAGNOSING DISEASE

C.-H. Liu, W.L. Glassman, H.R. Zhu, D.L. Akins, S.S. Lubicz * L.I Deckelbaum $^{b}$, J. Cleary ${ }^{c}$, R. Prudente ${ }^{c}$, E. Celmer ${ }^{c}$, and R.R. Alfano. CCNY, " La Guardia Hospital, ' Yale Uni. School of Medicine, 'Beth Isreal Medical Center.

Near-infrared(NIR) Raman spectroscopic technique has an unique advantage to study fluorescence-free biological macromolecules. NIR Raman spectroscopy may provide a new histological diagnostic technique for monitoring tumor growth and to separate malignant from nonmalignant biomedical media. Raman spectral measurements were performed on human tissues of breast, normal and atherosclerotic aorta and GYN tracts. The differences of Raman frequencies and their relative intensities for biomedical tissues are evidence between cancer, benign and normal tissues. The key Raman lines at $1659 \mathrm{~cm}^{-1}, 1445 \mathrm{~cm}^{-1}$, and $1262 \mathrm{~cm}^{-1}$ are assigned to the stretching vibration of $\mathrm{C}=\mathrm{O}$ (Amide $\mathrm{I}$ ), the bending vibration $\mathrm{C}-\mathrm{H}$, and the open stretching $\mathrm{C}-\mathrm{N}$ ( Amide III) respectively. The data displayed in the studies suggest that in muscle tissues, the changes in the molecules occur mainly in the protein elastin and the lipoprotein collagen. The change in relative intensity between Raman lines and the number of the Raman peaks may be used to separate cancer from nomal or benign tissues. Separation of calcified plaque using $\mathrm{PO}_{4}$ vibration line. The "optical Raman pathology" is defined from Raman cross section times the number of molecules i.e., $\sigma_{\mathrm{i}} \mathrm{N}_{\mathrm{i}}$. This work at CCNY was supported by Mediscience Technology Corp. and NSF.

199

FLUORESENCE SPECTRAL METHODS TO DETECT COLON CANCER TISSUE

"Yang Yuanlong, "G.C.Tang, "M.Bessler,"R.Alfano

Ingtitute for Ultrafast Spectroscopy and Lasers, The City college of New York, New York Spectroscopy

"Columbia University and The Columbia Presbyterian Medical

Two fluorescence spectroscopic methods have been used to distinguish between cancer and normal colon tissues using $C D$ scan cancer colon specimens,

The first optical method uses the intensity ratio of $360 \mathrm{~nm}$ to $380 \mathrm{~nm}$ excited by $325 \mathrm{~nm}$ to dist inguish between cancer and norma tissues. The ratio for most cancerous colon tissue is above 7 . while normal samples lie below 7.5 . When the ratio value 7.5 was used as a criterion to distinguish between cancer and normal tissues a consistency of $89 \%$ for normal and $90 \%$ for cancer was obtained as compared with pathologic examination.

The second method compares the fluorescence spectrum of each $t$ issue excited by $325 \mathrm{~nm}$ with an average spectral curve obtained by averaging all the individual fluorescence spectra for cancer and for normal tissues separately. According to the value of standard deviation of each specimen from the average curve, cancer was distinguished from the normal with 858 and $87 \%$ accuracy for cancer and normal tissue, respectively.

Combining the excitation and fluorescence spectra results, the origin of these differences is attributed to amount of tryptophan, collagen and elastin content excited and emitted from cancer and

This work is supported by Mediscience Technology Corporation. 
200

INTRACELLULAR SORTING OF EPIDERMAL GROWTH FACTOR RECEPTORS IN FIBROBLASTS French, A.R.*, G.P. Sudlow*, H.S. Wiley", D.A. Lauffenburger $*+$,

Depts. of ${ }^{*}$ Chemical Engineering and ${ }^{\dagger}$ Cell \& Structural Biology, Univ. of Illinois at Urbana-Champaign; "Dept. of Pathology, Univ. of Utah

Epidermal growth factor is an important regulator of proliferation of tissue fibroblasts. Attenuation of the proliferation signal occurs by receptor downregulation and ligand depletion arising from receptor/ligand trafficking processes. One key step in trafficking is endosomal sorting of receptors and ligands to alternative destinations of lysosomal degradation or cell surface recycling. Using a series of site-directed epidermal growth factor receptor mutants, we provide evidence that sorting to degradation is a stoichiometrically saturable process, involving specific interaction of an endosomal retention component with the receptor cytoplasmic domain. We further show that this interaction requires occupancy of receptor by its ligand. Quantitative understanding of this sorting process will help provide improved methodologies for regulation of cell proliferation in applications such as wound healing and tissue regeneration.

201 RECEPTOR-MEDIATED TARGET-FINDING BY NEURONAL GROWTH CONES
Helen M. Buettner and Julius f. Fernandez

Department of Chemical \& Biochemical Engineering

Rutgers-The State University of New Jersey, Piscataway, 08855-0909

The growth of pioneer neurites along specific pathways during nerve development or regeneration depends on receptor-mediated interactions between the growth cone at the leading tip of the neurite and molecular cues in the extracellula environment. These molecules are often concentrated at discrete regions along the growth path, forming a series of intermediate targets for the neurite to follow to its ultimate destination. In many cases, the distance between intermediate targets is large relative to the growth cone dimensions and to the characteristic distance of short term motion, which can be described as a random walk with drift. The effective sampling area of the migrating growth cone is increased by filopodia projecting from the growth cone periphery, but the filopodia structure is sparse random and dynamic, leading to a probabilistic sampling pattern. In the event of target encounter, target recognition depends on the binding of cell-surface receptor and extracellular ligand, also a probabilistic process. To provide insight into the way in which these stochastic events combine to accurately reproduce the paths of pioneer outgrowth observed in the embryo, we have developed a simulation model for growth cone target encounter and recognition. Filopodia dynamics are superimposed on the growth cone trajectory to simulate the growth cone sampling pattern and determine the probability of encounter; recognition is modeled in term of the probability of ligand-receptor binding. The model serves as a general framework for investigating receptor-mediated mechanisms of neurite guidance. Supported by NSF Grant BCS 92-10540; I.J.F. was supported by an REU Supplement.

\section{2}

EFFECT OF RECEPTOR AGONISTS/ANTAGONISTS ON [ $\left.\mathrm{Ca}^{2+}\right]_{\text {i }}$ MOBILIZATION DIGITAL FLUORESCENCE IMAGING AND MONTE CARLO MODELING Patricia A. Mahama and Jennifer J. Linderman

University of Michigan, Department of Chemical Engineering

Binding of ligand to receptors may activate a signal transduction pathway, resulting in an increase in intracellular free calcium, $\left[\mathrm{Ca}^{2+}\right]_{i}$. Activation of the signal transduction pathway is a result of receptor-ligand binding and 2-dimensional (2-D) diffusion and interaction of membrane proteins. Thus, drugs which interfere with protein movement or protein-protein interaction as well as receptor antagonists, or blockers, may alter or prevent signal transduction initiated by receptor-ligand binding.

We have monitored $\left[\mathrm{Ca}^{2+}\right]_{i}$ changes in phenylephrine ( $\mathrm{PhE}$ )-stimulated single $\mathrm{BC}_{3} \mathrm{H} 1$ smooth muscle-like cells using fura-2 in a digital fluorescence imaging system. In addition, we have pretreated single cells with the receptor antagonist prazosin $(\mathrm{Pz})$ and then $\mathrm{r}$ peated $\mathrm{PhE}$ stimulation to determine the effect of the receptor blocker on $\left[\mathrm{Ca}^{2+}\right]_{i}$ changes. We find significant cell-to-cell variability in the calcium responses of single $\mathrm{PhE}$-stimulated $\mathrm{BC}_{3} \mathrm{HL}$ cells. For the population-averaged calcium responses, we find that both the frac tion of cells which respond to $\mathrm{PhE}$-stimulation and the speed of the calcium response are increasing functions of ligand concentration for both $\mathrm{PhE}$ and $\mathrm{Pz}-\mathrm{PhE}$ stimulation. plexes on the cell surface, we find that cells pretreated with $\mathrm{Pz}$ are much less responsive than those stimulated with PhE alone.

We have used Monte Carlo models of receptor-ligand binding and 2-D protein diffusion to determine the expected effect of a receptor blocker on G-protein activation. Our Monte Carlo models predict two regimes of G-protein activation: 1) when the time-scale of receptor-ligand binding is slow compared to $2-\mathrm{D}$ protein diffusion or when the fraction of ligand-bound receptors is high and 2) when the time-scale of receptor-ligand binding is fast compared to 2-D protein diffusion and the traction of receptor-ligand binding is fast compared to 2-D protein diffusion and the fraction of ligand-bound receptors is response only in the second regime. Other results of the Monte Carlo models will also be discussed.
203

STOCHASTIC MODEL OF RECEPTOR PHENOMENA UNDERLYING LEUKOCYTE CHEMOTAXIS

Prabhas V. Moghe and Robert T. Tranquillo

Department of Chemical Engineering and Materials Science, University of Minnesota

Mammalian white blood cells such as neutrophil leukocytes are known to bias the direction of their movement along concentration gradients of specific chemical ligands, a phenomenon called chemotaxis. On a molecular level, these cells sense the ligands (called chemoatractants or ' $\mathrm{CA}$ ') via specific receptors which bind CA molecules. This event triggers a complex signal transduction process eventually culminating in observable cell response via activation of cell motility. Previous theory bas demonstrated that statistical fluctuations in receptor binding arising from a finite receptor number, can lead to 'noisy' signals which may explain the imperfect chemotactic response to $\mathrm{CA}$ gradient.

The most recent stochastic model (Tranquillo and Lauffenburger, 1987) couples simple intracellular signal transduction and cell motility response with fluctuating receptor binding. However, this model employs several assumptions for receptor dynamics that are now known to be oversimplificatons. Extensions to the aforementioned model are sought in now known to be oversimplificatons. Extensions to the aforementioned model are sought in physiologically meaningful receptor distributions. These include: a transient G-protein shysiologically meaningful receptor distributions. These include: a transient G-protein signalling state, a cytoskeletally-associated state, and receptor internalization and recycling,
including statistical fluctuations in numbers of receptors among the various states. The including statistical fluctuations in numbers of receptors among the various states. The
stochastic model is solved to yield simulations of cell paths and their statistics for various CA
gradient steepnesses. More importantly, the strength of the model lies in its ability to examine the effect of stochasticity of various receptor dynamics schemes on orientation behavior and reveal, successfully, mechanisms of adaptation behavior of cells. Several parametric sensitivity studies of analytically derived persistence time and numerically estimated orientation bias for a simulated cell population will be presented to highlight this feature.

\section{4}

ION CHANNEL REGULATION OF INSULIN SECRETION IN THE PANCREATIC BETA-CELL

M.-L. Kohler and C. L. Stoke

Department of Chemical Engineering, University of Houston, Houston, TX

Oscillations of the membrane potential of the pancreatic beta-cell help regulate the secretion of insulin by controlling the influx of calcium ions through voltage-dependent calcium channels. Both the oscillations and insulin release are graded responses that depend on blood glucose concentration, and the calcium influx associated with the oscillations are necessary for secretion to occur. The goal of our studies is to understand the regulation of the oscillations in membrane potential as part of the stimulus-secretion mechanism. Several potassium and calcium channels are involved in producing the oscillations. We have conducted experimental measurements on these channels using patch and voltage clamp techniques at more physiological conditions than ever previously used (body temperature and bicarbonate/ $\mathrm{CO}_{2}$ buffer compared to room temperature and hepes buffer). We have found significant differences in channel characteristics such as the single channel conductance and open probability under these conditions. Such differences may call into question previous conclusions about the involvement of various ion channels in the membrane potential oscillations and regulation of insulin secretion. The results of these experiments and analysis of their importance will be presented.

\section{5}

A MODEL OF THROMBIN-MEDIATED CYTOSOLIC CALCIUM MOBILIZATION IN HUVECS RESPONDING TO FLOW

Theodore F. Wiesner, Gabriel Helmlinger, and Robert M. Nerem

School of Chemical Engineering, Georgia Institute of Technology, Atlanta, GA 30332

Important among the responses of endothelial cells to flow stimuli are cytosolic free calcium transients. These transients are known to be mediated by several factors, including blood-borne agonists, extracellular calcium, and possibly shear forces. A mathematical model has been developed which simulates these transients as a function of an agonist. It has been applied specifically to the response of human umbilical vein endothelial cells (HUVECs) to a thrombincontaining medium in a capillary tube flow chamber. The conservation equations for momentum and agonist in the perfusate are coupled to the kinetic equations for receptor binding and mobilization of cytosolic $\mathrm{Ca}^{2}+$. Simulations of the time course of cytosolic $\mathrm{Ca}^{2+}$ mobilization compare reasonably to experimental measurements in cultured HUVEC. Parametric studies with the model indicate distinct contributions to the time course by each step in the process. Other computations indicate a flow region where the transient moves from transportlimited to kinetically-limited. 
206

\section{STRETCH-SENSITIVE WHOLE-CELL CURRENTS ARE ALIVE AND WELL F. Sachs, H. Hu SUNY, Biophysical Sciences, Buffalo, NY 14214}

It has been claimed that stretch activated ion channels (SACs) represent an artifact of patch clamping 1 \}. This conclusion came from experiments in which the authors could demonstrate

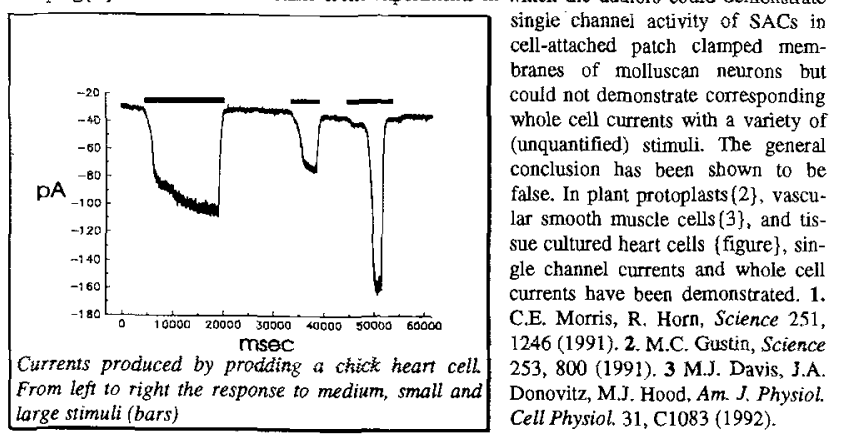

Supported by USARO DAAL0392G0014 and NSF 1BN9009675

207

MECHANOCHEMICAL TRANSDUCTION ACROSS THE CELL SURFACE AND THROUGH THE CYTOSKELETON: USE OF A TENSEGRITY MECHANISM.

Donald E. ingber, MD, PhD, James Butler, PhD, and Ning Wang, PhD.

Departments of Pathology \& Surgery, Children's Hospital/Harvard Medical School, and Respiratory Biology Program, Harvard Scbool of Public Health, Boston, MA 02115

How do cells sense physical forces and transduce mechanical information into a biochemical response? We have been exploring the possibility that the cellular response to force is based upon transfer of mechanical signals across specific transmembrane receptors that mediate cell attachment to extracellular matrix (ECM) and subsequent structural changes in the cytoskeleton. This concept is based on studies with cultured cells which demonstrate that cell shape and function can be controlled by varying the number of ECM attachment sites that can physically resist cytoskeletal tension. To test this hypothesis, mechanical stresses were applied directly to specific cell surface receptors without producing global cell sbape changes using a magnetic twisting device. The transmembrane ECM receptor, $\beta_{1}$ integrin, effectively transferred mechanical loads across the cell surface and supported a force-dependent cytoskeletal stiffening response whereas non-adhesion receptors did not. Force transfer correlated with recruitment of focal adhesion proteins and linkage of integrins to the actin cytoskeleton. Yet the cytoskeletal response to stress involved microtubules and intermediate filaments as well as microfilaments. Furthermore, the stiffness of the intact cytoskeleton increased in direct proportion as the level of applied stress was raised. This behavior was mimicked using "stick and string" tensegrity (tensional integrity) models that contain mechanically-interdependent structural elements that rearrange rather than deform locally in response to stress. These results demonstrate that integrins can act as cell surface mechanoreceptors and transmit mechanical signals to the cytoskeleton. Mechanochemical transduction, in turn, may be mediated simultaneously at multiple locations inside the cell by force-induced cytoskeletal rearrangements.
208

THE ROLE OF G-PROTEINS IN MECHANOCHEMICAL SIGNAL TRANSDUCTION

J. Nolan, S. Gudi, and J.A. Frangos

The Penn State University, University Park, PA 16802

It is now well established that numerous cells and tissues can transduce external physical forces into intracellular biochemical responses. It is not clear, however, how mechanochemical transduction occurs. While there have been several mechanisms proposed, few appear to completely account for all experimental observations. One proposed mechanism that accounts for transduction of physical signals, including shear stress, stretch, and electromagnetic fields, involves guanine nucleotide $(G)$ proteins and their associated membrane receptors. We have observed that pertussis toxinsensitive G-proteins mediate shear stress-induced gene expression, prostaglandin production and nitric oxide release in endothelial cells. We have also shown that these G-proteins are involved in mechanochemical signal transduction in osteoblasts. The role of $\mathrm{G}$-proteins and their associated receptors in mechanochemical signal transduction will be discussed.

209

THE INTRACELLULAR FREE CALCIUM RESPONSE IN ENDOTHELIAL CELLS SUBJECTED TO STEADY AND PULSATILE LAMINAR FLOW

Gabriel Helmlinger, and Robert M. Nerem, Georgia Institute of Technology, Atlanta, GA

There is indirect evidence suggesting that the intracellular free calcium ion concentration $[\mathrm{Ca} 2+]$ might play a second messenger role in the recognition of a fluid-imposed shear stress by endothelial cells (ECs). We measured $\left[\mathrm{Ca}^{2+}\right]_{\mathrm{i}}$ in cultured bovine aortic ECS (BAECs) subjected to various steady and pulsatile (non-reversing, reversing, and oscillatory) laminar flows, using different flow media (plain DMEM and DMEM $+10 \%$ calf serum), digital imaging and ratiometric techniques. Steady flows of various levels and non-reversing and reversing pulsatile flows elicited $\left[\mathrm{Ca}^{2}+\right]_{i}$ increases in $\mathrm{EC}$ monolayers, the amplitude and rising time of which were dependent on the flow and media conditions. For all media conditions, there was no $\left[\mathrm{Ca}^{2+}\right]_{i}$ increase in monolayers subjected to an oscillatory type of flow. Individual cell analyses revealed asynchronous $\left[\mathrm{Ca}^{2+}\right]_{i}$ oscillations for all flow conditions. The total number of such oscillations within a monolayer increased with the level of steady flow. It was of major interest to observe that oscillatory flow could induce, for any medium condition, asynchronous individual cell $\left[\mathrm{Ca}^{2+}\right]_{i}$ oscillations without yielding an overall monolayer response. These results show that ECs are able to respond to different flow environments in terms of $\left[\mathrm{Ca}^{2+}\right]_{\mathrm{i}}$ signaling. 
210

SCROLL WAVE DYNAMICS

James $P$. Keener

Department of Mathematics, University of Utah, Salt Lake City, UT 84112

Scroll waves are the three dimensional extensions of the rotating spiral waves seen in a variety of excitable media. Scroll wave dynamics are important to an understanding of ventricular tachycardia and sudden cardiac death syndrome. In this talk we describe some analytical and numerical features of scroll wave dynamics and their implications to understanding fatal cardiac arrhythmias. Results of numerical simulations will be shown in a video presentation.

\section{1}

MATHEMATICAL MODELS OF BLOOD FLOW IN THE MICROCIRCULATION T.W. Secomb

Dept. of Physiology, Univ. of Arizona, Tucson AZ 85724

In most organs, the microcirculation consists of an extensive and intricate network of very narrow tubes. Blood is a concentrated suspension, containing about $45 \%$ by volume of red cells, with unstressed diameters that are typically larger than the diameters of the smallest capillaries. For more than twenty- five years, mathematical models have been used to study the mechanics of blood flow in the microcirculation, and to predict physiologically important parameters such as flow resistance and oxygen transport. The basic mechanical properties of individual mammalian red cells are known and provide a starting point for models. A homogeneous viscous fluid interior is surrounded by a flexible membrane, which strongly resists area changes, but bends and shears easily. In capillaries, the flow of suspending fluid (plasma) in the narrow gaps between the cells and the vessel walls may be described using lubrication theory. Resulting predictions of flow resistance, assuming axisymmetric cell shapes, agree with determinations in narrow glass tubes. Red cells flowing in microvessels typically assume non-symmetric shapes with cyclic "tank treading" motion of the membrane around the interior. Mathematical models including these effects suggest that they have little effect on flow resistance in capillaries. At diverging microvascular bifurcations, uneven partition of red cells and plasma often results in different hematocrits in the two daughter branches. Simulations of blood flow in networks must take this effect into account, as well as including the effect of vessel diameter and hematocrit on flow resistance. Comparisons between results of such simulations and observations of blood flow in networks provide a critical test of current understanding of blood flow in the microcirculation. Supported by NIH Grant HL34555.
212

A DYNAMIC NUMERICAL METHOD FOR MODELS OF RENAL TUBULES E. Bruce Pitman, Dept. of Mathematics, SUNY at Buffalo, Buffalo, NY 14214 Harold E. Layton, Dept. of Mathematics, Duke University, Durham, NC 27706

In this report we describe the application of a modern numerical method for solving hyperbolic partial differential equations to calculating solute concentrations in a renal tubule. Specifically, we implement an ENO (Essentially Non-Oscillatory) method that is second-order accurate in space and time. Implementation of this method eliminates numerical instability arising from transient reversal of flow direction, a common problem with other methods. We present examples of computed flows and concentration profiles in representative model contexts. We indicate how model tubules may be conjoined and coupled to construct large-scale simulations of the renal counterfow system, including recently measured nephron permeabilities.

\section{3}

MODELING OF PLATELET AGGREGATION

Aaron L. Fogelson

Department of Mathematics, University of Utah, Salt Lake City, UT 84112

We will present an approach to modeling and simulating platelet aggregation in arteriolesized vessels. The model involves descriptions of dynamic mechanical and chemical interactions among platelets, a suspending fluid, a platelet activating chemical, and a reactive wall. The model tracks the motion and behavior of individual platelets, including their possible adhesion to reactive portions of the vessel/prosthesis wall and cohesion to one another. Initially-nonactivated platelets may become activated through contact with reactive sites on the wall, or through exposure to a sufficiently high concentration of the activating chemical in the fluid. Upon activation, a platelet becomes capable of cohering to other activated platelets, and it secretes additional activating chemical into the surrounding fluid. Mechanical interactions among platelets, the fluid, and the vessel wall are handled using the Immersed Boundary Method. This is a general computational technique for studying interactions between a viscous incompressible fluid and immersed elastic objects. In the aggregation context, it allows us to study two-way interactions between aggregate growth and fluid dynamics. In particular, it permits study of embolic events and thrombus growth up to and including lumen closure. We will show a video of our computational simulations. 
214

ANALYZING MULTI-PARAMETER MODELS USING VISUALIZATION Sorel Bosan, Thomas R. Harris

Department of Biomedical Engineering, Vanderbilt University, Nashville TN, 37235

A visualization method was developed for observing the effects of parameter variations on multi-parameter models. The method was based on a visualization tochnique which allows the viewing of n-dimensional surfaces as either 3-dimensional surfaces or 2dimensional images. For illustrative purposes, the method was used for analyzing the behavior of a three parameter effective diffusivity model of pulmonary capillary exchange. Each model parameter, as well as time, was assigned a dimension. Then, a 5-dimensional surface, consisting of model outputs as a function of time and the three model parameters was generated. Next, the 5-dimensional surface was mapped to and plotted as a 2-dimensional image. We repeated the above steps for different ranges of parameters. The resulting images enabled us to observe the model's behavior with respect to its parameters at a glance. Whereas, previously, information about the model's behavior was obtained only implicitly in the form of a mental picture, our method made this information explicitly and readily available. Furthermore, whereas the previous method of forming implicit mental pictures required the modellers to go through the process of running large numbers of simulations, guided only by intuition, our visualization-based method automated this process, greatly speeding up model analysis. Because of these advantages we conclude that our method, which enables the instant observation of a model's behavior with respect to its parameters, is a significant addition to the formal tools of model analysis.

Acknowledgment

This work was supported by NIH Grant RR06558-03

215

MODELING OF HYFEFTHOPITY DUE TO REDUCED MYOCARDIAL CONTRACTILTY G. Orzewiecki ano .J.K-J. Li

Cardiovascular Research Labs, Biomedical Eng. Dept., Rutgers University, NJ

A mathomatical mcdel of cardiac hypertrophy has been develoned. In this study, the model is applied to study cardiomyopathy. The model defines a myofibril unit as the basic building block of the left ventricle, whose function is specified. Myofibril units are arranged in parallel and series, assuming a cylindrically shaped chamber. The number of series and parallel fibril units and heart rate are then solved using the following criterion. The rate of myocardial oxygen consumption must be both minimal and in equilibrium with that supplied under chronic conditions. This model has successfully demonstrated the concentric and eccentric forms of hypertrophy that arise due to pressure and volume overloads, respectively.

Reduced myofibril contractility was introduced by lowering the value of the maximum stress-length slope. This is somewhat analogous to maximum elastance of the ventricle but at the cellular level. Blood pressure and cardiac output were required to be constant, i.e. fully-compensated hypertrophy. It was found that the concentric type of hypertrophy developed as the fibril unit contractility was progressively lowered. A $10 \%$ decrease in contractility resulted in a nearly $20 \%$ increase in wall thickness. In addition, heart rate was increased while stroke work, ventricular efficiency, and fiber stress were diminished. Further decreases in contractility produced excessively large heart rates, indicating loss of cardiac reserve and onset of failure. These results are consistent with clinical cardiomyopic hypertrophy.

\author{
A Mathematical Model of the Canine Circulation \\ D. C. Chung ${ }^{\dagger}$, J. W. Clark Jr. ${ }^{\dagger}$, A. Bidani ${ }^{\ddagger} \&$ D. L. Traber ${ }^{\ddagger}$ \\ Rice University, Houston, Texas $77251^{\dagger}$ and \\ University of Texas Medical Branch, Galveston, Texas $77550^{\ddagger}$
}

A lumped-parameter model of the canine circulation, which includes both the systemic and pulmonary circulations, has been developed. The model consists of the following components: the right heart (atrium and ventricle), pulmonary circuit (pulmonary arteries, capillaries, and veins), left heart (atrium and ventricle), aorta systemic arteries, systemic veins, and the vena cavae. The passive elements, the arteries and veins, are represented by resistive, compliant, and inertial segments similar to those of previous studies. In addition to the mathematical description of the arteries and veins of both the systemic and pulmonary circulations, this model makes use of the elastance (instantaneous pressure-volume relationship) of the ventricles. Adopting elastance functions for both the left and right ventricles, the model produces realistic left and right ventricular pressure and volume waveforms. Model parameters associated with the systemic and pulmonary circulations have been adjusted so that the pressure waveforms at various points in the circulation agree quite well with data from animal experiments. Furthermore, the model yields good estimates of cardiac output and ejection fraction for the normal dog. The model is capable of mimicing the response of the cardiovascular system to a number of experimental manipulations (e.g. response to vasoactive drugs).

This work is supported by the Biomedical Engineering Center at the University of Texas Medical Branch, Galveston, TX.
217

SURROGATE DATA ANALYSIS OF MICROVASCULAR BLOOD FLOW DYNAMICS D.R. Sainanitł, C.G. Ellist, T. Lookmane 'Dep'ts of Medical Biophysics, 'Electrical Engineering, 'Applied Mathematics The University of Western Ontario, London, Canada

Capillary red blood cell (RBC) flow in the extensor digitorum longus muscle in the hindtimb of male wistar rats was videotaped for periods of at least 10 minutes using intravital video microscopy. Time series analysis was used to analyse the dynamics of the $\mathrm{RBC}$ velocity records, and the autocorrelation function, correlation dimension and Lyapunov exponent were all computed for the data. While the results from these analyses demonstrate the existence of a strange attractor of finite dimension and small positive Lyapunov exponents, a clear characterization of the dynamics as Chaotic was not possible. It is possible that other types of dynamics could generate similar behaviour. Using a new method from the field of non-linear dynamics, an ensemble of "surrogate datasets" were constructed from the original in-vivo RBC velocity data. These surrogate datasets share some characteristics with the original data, such as the power spectrum, but are essentially randomly distributed data. These surrogate datasets permit us to distinguish whether the dynamics of a system are due to chaotic processes, linearly correlated noise, or non-linear transformations of a stochastic process. Time series analysis was again used to determine the dynamic characteristics of the data and the discriminating statistics used (dimension or Lyapunov exponent etc.) were compared for both the surrogate data and the original dataset. The results from the data analysed to date, point to a characterization of the dynamics of capillary red blood cell flow as non-linear transformations of stochastic processes in some cases, and in other cases as linearly correlated noise.

This study was supported by the Heart \& Stroke Foundation of Ontario

Dr. Ellis is a Career Investigator of the Heart \& Stroke Foundation

218

SHOCK WAVE PROPAGATION IN RENAL CALCUU WTTH LAYER STRUCTURE

Pei Zhong, C.J. Charles Chuong, Glenn M. Preminger

Division of Urology and Biomedical Engineering Program

The University of Texas Southwestern Medical Center at Dallas

To understand the mechanisms of stone fragmentation during extracorporeal shock wave lithotripsy, a theoretical model has been developed to analyze the transient impingement of cavitation microjets on renal calculi and the resuitant shock wave propagation inside the stones based on geometrical acoustics. [JASA, 1993. (in press)]

This model is used to calculate jet impact pressure at the impacting surface as well as stress and strain discontinuities at the propagating shock fronts in stones of various compositions. Shock reflection and transmission at interface of stone crystal and matrix were determined and the effects of stone size, layer thickness and compositions on the resultant shock propagation were examined. Model calculation under typical lithotripsy conditions predicted compressive stresses of $629 \sim 2,517 \mathrm{MPa}$ and shear stresses of 81 $\sim 324 \mathrm{MPa}$ at the impacting surface, and a tensile stress up to $7.0 \mathrm{MPa}$ at the distal surface of the stone. Comparing with corresponding failure strength of the stone material, the model calculations predicted cavitation erosion at anterior and spalling damage at posterior surface of the stone as observed from stone fragmentation experiments.

The modeling results also show that reflected tensile waves are generated when incident compressive waves reach the crystal-to-matrix boundaries. The intensity of the reflected tensile wave is generally higher for smaller stones and at layer interface closer to the anterior surface of the stone. These results suggest that stones with concentric layer structure are more prone to tensile failure (layer separation) and they are more fragile than homogeneous stones under the same shock wave intensity. These results may help to improve the treatment efficacy of stone removal using shock wave lithotripsy.

\section{9}

THE INFLUENCE OF MODELING ON FINITE ELEMENT RESULTS

C. McMahon, Dr. T. McGee, Dr. T. Rogge, G. Niederauer, R. Thielman. Iowa State University, Ames, Iowa.

Stress analysis of dental implants is often performed using a two dimensional (2-D) finite element analysis of models that represent cancellous bone as homogeneous. The models use a coarse mesh and often differ from one another without explanation in their choice of boundary constraints. This study uses a 2-D finite element analysis to investigate how cancellous bone modeling and the choice of boundary constraints affect the stress and strain distribution around a dental implant. The degree of mesh refinement required for an accurate 2-D analysis is also determined using an h-convergence test. Three 9500 node, 2-D, plane strain finite element models using 8-noded isoparametric quadrilateral elements were created from a scanned image of the central slice of a retrieved implant. Each model represented the cancellous bone in a different way: completely homogeneous, partially inhomogeneous and inhomogeneous with the entire cancellous structure being represented in the model. Preliminary results indicate that the choice of boundary constraints as well as modeling the cancellous bone as homogeneous versus modeling its true inhomogeneous structure results in significant differences in both the magnitude and distribution of stress and strain in the bone around the implant. The convergence test indicates that a $3000-4000$ node mesh is required for an accurate 2-D finite element analysis. 
220

EFFECTS OF BLOCKING THE PLATELET GP Ib INTERACTION WITH VON WILLEBRAND FACTOR (WWF) UNDER A RANGE OF SHEARING FORCES Christos P. Markou, Anne K. Lindahi*, I. M. Siegel, David N. Ku, Stephen R. Hanson* School of Mechanical Engineering, Georgia Institute of Technology, Atlanta, GA 30332 "School of Medicine, Emory University, Atlanta, GA 30322

Interactions between platelet receptors and adhesive plasma glycoproteins regulate the development of arterial platelet thrombl, and may be highly shear dependent. To study this process a recombinant $v W F$ fragment (VCL: Leu504-Lys728), which blocks the GP Ib binding domain for VWF was evaluated under different shearing conditions in vivo. Platelet deposition on collagen coated straight tubes and axisymmetric stenoses models exposed to nonanticoagulated blood $(100 \mathrm{ml} / \mathrm{min})$ in a baboon AV shunt system was measured by ${ }^{111}$ Indium-platelet imaging. Results after 20 min exposure were

Average wall Average wall $\quad$ Controls Platelets $\left(x 10^{9}\right)$ deposited $/ \mathrm{cm}^{2}$

$265(4 \mathrm{~mm})$ Controls

$630(3 \mathrm{~mm})$ $2.31 \pm 0.28$ $5.96 \pm 0.71$ $1.91 \pm 0.2$ Significance

$900(50 \%)$ $16.51 \pm 1.89$ $4.04 \pm 0.28$ $3.78 \pm 0.62$ $22.06 \pm 2.7$

$7.46 \pm 0.79$ $24.59 \pm 1.38$

$8.20 \pm 2.38$

$2400(75 \%)$ $33.71 \pm 2.21$ $7.00 \pm 0.84$

Values are mean $\pm S E ; n \geq 6$ in each group; ${ }^{N S}, p>0.05$ )

SE; $\geq 6$ in each group; $N 5, p>0.05$ ) Platelet deposition increased markedly with shear rate. For the $2 \mathrm{~mm}$ tubes and the $75 \%$
and $90 \%$ stenoses occlusion occurred within $25 \mathrm{~min}$. of blood exposure. VCL produced and $90 \%$ stenoses occlusion occurred within $25 \mathrm{~min}$. of blood exposure. VCL produced
profound inhibition of thrombosis at shear rates $>900 \mathrm{~s}^{-1}$; these effects persisted throughout the $2 \mathrm{hr}$ study period and no occlusions were observed. These results demonstrate that blocking the GP $\mathrm{lb}$ binding domain for $\mathrm{VWF}$ using a rvWF fragment in vivo can inhibit thrombus formation at sites of high shear, such as the apex of stenoses.

\section{1}

INHIBITION OF PLATELET THROMBUS FORMATION IN FLOWING BLOOD BY VON WILLEBRAND FACTOR AND GP Ib ANTAGONISTS

BR Alevriadou, J Dent, R McClintock, J Ware and ZM Ruggerj

The Scripps Research Institute, La Jolla, CA

Previous studies have demonstrated that a reduced and alkylated recombinant fragment of von Willebrand factor (vWF), comprising residues $445-733$ and corresponding to the binding site for the platelet receptor glycoprotein (GP) $\mathrm{Ib}$, inhibits platelet adhesion from flowing blood onto collagen coated surfaces under arterial flow conditions. Two cysteine residues (positions 509 and 695) form an intrachain disulfide bond within this region of vWF. Binding assays suggest residues 508 to 709 may be the minimal structure retaining affinity for GP Ib, and that formation of the 509-695 disulfide bond may be important in modulating the binding. In this study, we have used a parallel-plate flow chamber to study thrombus formation onto collagen-coated surfaces by perfusing fluorescently-labelled platelets in whole blood containing PPACK (a thrombin inhibitor) as anticoagulant. A laser scanning confocal workstation, consisting of an inverted epifluorescent microscope with an argon laser, was used for visualization with improved resolution of the thrombi formed. Surface topography software was employed to reconstruct the thrombi structures in $3 \mathrm{D}$ and measure their volume and surface coverage. A recombinant vWF fragment composed of residues 508-709 was expressed and purified in both the oxidized (with an intact 509-695 disulfide bond) and the reduced and alkylated states. Both forms inhibited in a dose-dependent manner platelet accumulation from whole blood onto collagen-coated surfaces with an $\mathrm{IC}_{50}$ of $\sim 3 \mu \mathrm{g} / \mathrm{ml}(\sim 130 \mathrm{nM})$ at $1,500 \mathrm{~s}^{-1}\left(60 \mathrm{dynes} / \mathrm{cm}^{2}\right)$. A monoclonal antibody against the GP lb-binding site in VWF inhibited platelet thrombus formation with an $\mathrm{IC}_{50}$ of $\sim 0.3 \mu \mathrm{g} / \mathrm{ml}(\sim 6 \mathrm{nM})$. When blood was incubated with both the receptor and the ligand antagonists, there was an additive effect on the inhibition of platelet accumulation onto collagen. These results provide information on the minimal structural requirements for VWF/GP Ib interaction, the initiation of arterial thrombosis, and may help design a new class of antithrombotic agents.

222

INHIBITION OF MURAL THROMBOSIS BY A CARBAMOYL-PIPERIDINE CONGENER Tabish N. Zaidi ${ }^{1}$, Larty V. Mcintire ${ }^{1}$ and R. Gollamudi ${ }^{2}$

${ }^{1}$ Cox Laboratory for Biomedical Engineering, Rice University, Houston, TX

${ }^{2}$ Health Science Center, The University of Tennessee, Memphis, TN

We have investigated the inbibitory potency of one of the most promising compounds in the list of carbamoyl-piperidine members, called A12, on type I bovine collagen induced mural platelet aggregate formation, over a wide range of whole blood concentrations and volumetric flow rates. A parallel plate flow chamber was employed to simulate kinematically a damaged blood vessel. The experimental conditions were monitored to be as close to physiological situation as possible. Heparinized human blood with fluorescently labelled platelers was perfused over collagen coated glass at $37^{\circ} \mathrm{C}$. Epifluorescent video microscopy and digital image processing enabled us to study the end point platelet accumulation as well as kinetics of indivioually growing thrombi with resolution of two components of thrombosis, viz. platelet-wall interaction (adhesion) and platelet-platele interaction (aggregation) made possible by the later technique. Both adhesion and aggregation were impaired in the presence of A12 in a dose dependent manner, reflecting that the compound acted comprehensively, presumably by stabilizing lipid membranes. At a wall shear rate of $800 \mathrm{~s}^{-1}$ the aggregation at the end of 2 minute perfusion was inhibited by $94 \%$ with $50 \mu \mathrm{M}$ concentration of $\mathrm{A} 12$ in who compound inhibited total platelet accumulation by $56.8+5.0 \%$, and by $79.2+4.3 \%$ at $50 \mu \mathrm{M}$, wall shear rate being $800 \mathrm{~s}^{-1}$. At blood A12 level of $21.5 \mu \mathrm{M}$, the percent inhibition with respect to controls was $52.3+6.2 \%$ and $61.1+6.8 \%$ for wall shear rates of $250 \mathrm{~s}^{-1}$ and $1500 \mathrm{~s}^{-1}$ respectively. This difference was, however, not statistically significant. Preliminary toxicity studies are underway for the carbamoyl-piperidine family, and results are very promising.
223

ROLE OF THE VWF-AI DOMAIN IN PLATELET ADHESION UNDER CONTROLLED FLOW CONDITIONS

G. A. Barabino, B. Zhang, S. Narins, M. A. Cruz, B. M. Ewenstein, R, J. Wise, and $R$. I. Handin

Department of Chemical Engineering. Northeastern University. Division of Hematology-Oncology, Brigham \& Women's Hospital, Boston, MA.

von Willebrand factor (vWF) serves as a molecular bridge between platelets and the subendothelium at sites of vascular injury. For stable platelet adhesion at high shear rates. vWF must bind to the glycoprotein lb/IX (GPIb/IX) receptor on the platelet surface. Further platelet spreading and attachment is facilitated by the platelet GPIIb/IIIa receptor. This work characterizes the attachment of washed human platelets to purified vWF molecules immobilized on glass under controlled flow conditions. Either recombinant wild type or plasma derived-vWF was coated onto glass slides which formed the base of a parallel plate flow chamber. vWF-coated slides were perfused with reconstituted blood at a shear rate of $1500 / \mathrm{sec}$ and platelet attachment was quantified using video microscopy. Both wild type recombinant and plasma vWF supported signtficant platelet adhesion, compared to BSA coated control slides $(6468 \pm 1010$ platelets $/ \mathrm{sq} \mathrm{mm}$ vs. $58 \pm$ 53 platelets $/ \mathrm{sq} \mathrm{mm}$ ). In order to assess the relative importance of the GPIb 53 platelets $/ \mathrm{sq} \mathrm{mm}$ ). In order to assess the relattve importance of the GPIb
binding domain, contained within the vWF-Al domain, we've expressed a binding domain, contained within the vWF-Al domain, we've expressed a
recombinant $\mathrm{VWF}$-Al fragment (residues 474-709) in Chinese hamster ovary (CHO) cells. This fragment purified to homogeneity by one-step of ton exchange chromatography had a molecular weight of 39,000. The vWF-AI fragment inhibited ristocetin-dependent platelet agglutination, but did not support platelet binding in the flow system, suggesting the possibility of its use as a potential anti-thrombotic agent.

\section{4}

MODEL SYSTEMS OF THROMBUS FORMATION IN FLOWING HUMAN BLOOD AT THE APEX OF ECCENTRIC STENOSES

Kjell S.Sakariassen and R.Marius Barstad

Nycomed Bioreg AS, Oslo, Norway.

Thrombus formation at ruptured stenotic plagues is often associated with life-threatening episodes. The mechanisms of thrombus formation at such lesions are not well understood, but the particular blood flow conditions introduced by the stenosis and the thrombogenic components of the fissure, e.g. collagens and tissue factor, are apparently playing important roles.

We have developed model systems to study thrombus formation in native human blood at the apex of eccentric stenoses. The thrombus formation is triggered by either collagen fibrils or tissue factor at wall shear rates varying from 2.600 to $32.000 \mathrm{sec}^{-1}$. The introduction of an eccentric stenosis enhances the thrombus formation two-fold when compared to thrombus formation at similar shear conditions without the inclusion of a stenosis. The deposition of fibrin is particularly pronounced in thrombi formed at the stenotic apex. However, thrombus formation in blood from patients with low levels of FVII coagulant activity $(1.3-4.5 \%)$ is significantly reduced. In contrast, aspirin ingestion by healthy volunteers has much less reducing impact on thrombus formation than deficiency of FVII.

Thus, it appears that a stenotic lurninal outgrowth enhances the thrombus formation significantly. Thrombin generation at the evolving thrombus may play a significant role in this event.

225

ROLE OF hEMOdYNAMICS IN PLATELET STIMULATION IN SEVERELY STENOSED CAROTID ARTERIES

Boreda, R., Fatemi, R.S., and Rittgers, S.E., Dept. of Biomedical Engineering, Univ. of Akron.

Recent clinical studies have shown a high incidence of thrombus formation around severe arterial stenoses. Thrombosis superimposed on the already existing atherosclerotic lesion is known to contribute to a majority of cardiac and stroke related deaths. Flow disturbances caused by high grade atherosclerotic lesions are thought to be contributing factors in initiating and accelerating a thrombus formation. The present study was undertaken in an effort to assess the role of abnormal hemodynamics existing in the vicinity of a severe stenosis in the stimulation of platelets

An in-vitro experiment was performed to study and quantify the flow patterns downstream of a stenosis using an ultrasound Doppler Color Flow Imaging (DCFI) system. Compliant arterial models were fabricated with different degrees $(51.2,63,72.5,71$ and $79.6 \%$ area reduction) of symmetric and asymmetric stenoses. These models were placed in a pulsatile flow loop and a blood analog fluid was circulated (Mean $R e=400, \alpha=5.3$ ) to simulate carotid artery flow. Flow images were recorded in real-time up to three diameters downstream of a stenosis using a 128 channel, 7.5/5 MHz linear array transducer. These images were digitized, uploaded and later decoded into velocities using a color image processing set up. The velocity data from jet region at peak systole was used to cbtain a) shear stress at each point in the fiow feild, and b) exposure time for particles. Shear stress was plotted against exposure time to assess the potential of different degrees of stenoses in activating platelets.

Results show that, shear stresses and particle exposure times vary with the degree and shape of stenosis. As the severity of stenosis increases, shear stresses attain higher values and exposure times become shorter. Based on the magnitude of shear forces known to activate platelets (Hellums ot al, Studies on Shear Induced Platelet Activation, Cerebral Ischemia and Blood Rheology, 87 ), it was found that in $71 \%$ or lesser degree of asymmetric stenosis and $79.6 \%$ or lesser degree of symmetric stenosis, shear stresses do not reach critical levels. Extrapolation showed that these parameters would attain critical levels when the degree of stenosis is in the range of $90-95 \%$ 
226

AUGMENTED MASS TRANSPORT IN SHEARED SUSPENSIONS OF RED BLOOD CELL GHOSTS

W. Cha and R. Beissinger, Illinois Institute of Technology, Chicago, IL 60616

Significant mass transport of macromolecules to surfaces in cellular suspensions undergoing laminar shear flow occurs in various biological systems. Both the dispersive and rotational motions of the suspended particles may augment the Brownian diffusivity of the macromolecular solutes. Shear-induced mass transport of albumin in a particle suspension of red blood cell (RBC) ghosts was determined by measurement under diffusion-limited conditions of macromolecular adsorption rates to the glass wall of our rectangular flow system. Adsorption was measured continuously using total internal reflection fluorescence techniques for suspension particle volume fractions $(\Phi)$ of $51070 \%$ and shear rate $(\gamma)$ of 200 to $1000 \mathrm{~s}^{-1}$. The effective diffusivity of albumin was found to be enhanced compared to the Brownian diffusivity value measured for the homogeneous protein solution. Augmentation of protein transport (defined as $D_{j} D_{s}-1$, where $D_{e}$ and $D_{s}$ are the effective diffusivities under flow and stationary condition, respectively) was found to increase from 17 to $1300 \%$ as $\Phi$ and $\gamma$ were increased to the maximum values used in the study. Augmentation normalized by the particle Peclet number was found to increase with $\Phi$ to a maximum at $45 \%$ (as a result of the normalization) for all shear rates tested. Shear-induced particle diffusivity ( $D_{p}$ ) was also determined from the augmented protein transport results. Based on a flow model, the effective diffusivity of the macromolecular solute was determined to be equal to $D_{s}$, i.e., the diffusivity of solute in suspension without flow, plus $D_{p}$, i.e., the shear-induced particle diffusivity. The shear-induced particle diffusivity $\left(D_{p}\right)$ was determined based on the augmented protein transport experimental results. Values of $D_{p}$ normalized by shear rate were observed to reach a maximum value $\left(2.2 \times 10^{-6} \mathrm{~cm}^{2} / \mathrm{s}\right)$ at a $\Phi$ of $45 \%$. The particle diffusivity values as correlated with $\Phi$ were found to be in good agreement with that in the literature.

\section{7}

PHYSICAL EXPLANATIONS OF NEAR-WALL EXCESSES OF PLATELETS E. C. Eckstein, University of Tennessee, Memphis, Dept. of Biomedical Engineering, 899 Madison Avenue, Suite 801, Memphis, TN 38163

The initial explanation for large near-wall excesses of platelets, which are observed at wall shear rates of 400 inverse seconds and above, involved a mechanism used to describe the shearthinning property of blood viscosity. At high shear rates, destruction of rouleaux was hypothesized to lead to a lessening of the available volume in the core region; as each red cell separated from the rouleaux, the available volume in the core would be reduced by an amoun equal to the excluded volume around the individual red cell. For small amounts of available core volume, there would be a high platelet concentration, leading to a movement to the wall region, which was assumed to be relatively unchanged at various wall shear rates. Close inspection of the explanation revealed two defects; the method of estimating the excluded volume pertained to dilute solutions, which is not appropriate for the near-wall excesses, and the statistical basis for the calculation was specially posed. These defects are specially problematic at high concentrations where near-wall excesses have also been observed. Their correction would lead to a model that is exceedingly difficult to evaluate, and therefore, relatively unsatisfying as a physical explanation.

A combination of other microrheological events, principally, alignment of the flexible red blood cells in shear flows and flow-induced lateral motion away from boundaries, may provide a suitable explanation. Such events suggest that the random motion would not be uniform. The effects of imposed forces or induced drift can be included in random walks. Including such phenomena leads to forcing terms such as were used in an extended convective diffusive equation (E.C. Eckstein, F. Belgacem, Biophys. J., 60:53-69, 1991). That model estimated the forcing (drift) temn from the steady state, fully developed concentration profile. Physically, the above mentioned microrheological events would act on the otherwise randomly moving particles to establish the fully developed profile.

\section{8}

PARTICLE TRAJECTORY PATTERNS AND RESIDENCE TIMES DOWNSTREAM OF A CRITICAL ARTERIAL STENOSIS

Cao, J., Fatemi, R.S., and Rittgers, S.E., Dept. of Biomedical Engineering, Univ. of Akron

Severe thrombotic events frequently occur with critical stenoses of human left anterior descending (LAD) coronary arteries and internal carotid arteries (ICA). Mechanical Stimulation of platelets at the narrowed region of these arteries in conjunction with the longer residence times downstream may lead to platelet adhesion and thrombus formation.

An in vitro flow visualization technique has been developed to quantify the flow pattems and compute platelet residence times downstream of a stenosis. Pulsatile LAD and ICA waveforms were applied to $75 \%$ and $95 \%$ area reduction asymmetric silastic models. Neutrally buoyant platelet analog particles (amberlite) were introduced into the flow field illuminated by a chopped laser (300mW Argon-ion). Particle trajectories were recorded by a high resolution monochrome CCD camera operating at frame integration interlaced mode, and a pseudo-colo display of the flow directions and platelet residence times were obtained by using a PC based image processing package. The software written in $\mathrm{C}$ language includes the procedures of thresholding, pattern recognition, gravity center calculation and pseudo-color display. Furthermore, $99 \%$ particle washout intervals were calculated by injecting particles upstream and counting them at the end of each pulse cycle over four diameters downstream.

Complex flow patterns including jetting and separation vortices were found downstream of the stenoses. Platelet residence times were higher for the LAD vs. the ICA and for the $95 \%$ stenoses vs. the 75\% stenoses, In addition, $99 \%$ particle washout intervals (PWI) illustrated that: PWI $(95 \% / L A D)>$ PWI $(75 \% / L A D)>$ PWI $(95 \% / I C A)>$ PWI(75\%/ICA $)$. These results indicate that coronary flow conditions and critical stenoses favor accumulation of platelets and formation of arterial thrombosis.
229

NUMERICAL SIMULATION OF THROMBUS FORMATION IN STENOSED CORONARY

ARTERIES,
Acharya S., Rittgers S.E., Mugler D.H., Dept. of BME, University of Akron.

Atherosclerosis in combination with thrombotic occiusion, is a major cause of deaths in United States. Even though these and related phenomena have been extensively studied, the exact chain of events in thrombotic occlusion, is not completely known. In particular, the details of how abnormal hemodynamics in stenosed arteries may lead to piatelet activation and consequent thrombogenesis have not been established. The purpose of this study is to evaluate the sthear stress - exposure time history and near wall residence times of platelets passing through a severely stenosed artery

A 2-D. Navier Stokes simulation of flow in an asymmetric stenosed artery (Area eduction $=75 \%, 90 \%, 95 \%$ ) was carried out on a commercially available finite element package for input coronary arterial conditions ( $\alpha=2.3, R_{m}=87, R_{p}=220$ ) and Newtonian fluia. Particle paths representing platelet trajectories were tracked through the stenosis. The shear stress exposure time history along a path was obtained. The data was then compared with critical shear - exposure times to determine if the platelet analogs would be stimulated [1]. Statistical indices for correlation between severity of stenosis and probability of platelet activation were calculated. Results of this study show complex flow patterns in the stenosed region with jetting at the throat. The jet bends towards the stenosed wall causing a wake of vortices. For the mor severe stenoses, we observed: (i) The shear stresses near the crown of the stenosis are increased and corresponding exposure times are decreased. (ii) Vortices have larger size and corresponding residence times are higher

The results of this study, are useful in determining the probability of platelet activation in stenosed coronary arterial conditions. They aiso establish the likeilihood for platelet adherence to the vessei wall and resulting thrombus formation downstream of the stenosis. This will be important in identifying a critical stenosis clinically

1. Hellums et al, Studies on Mechanisms of Shear Induced Platelet Activation, Cerebral Ischemia and Hemorheology, 1987.

230

INNNER CLOT PERMEATION CONTROLS RATE OF THROMBOLYSIS Sriram Anand, Jung He Wu, and Scott L. Diamond Bioengineering Laboratory, 907 Furnas Hall, S.U.N.Y., Buffalo, NY 14260

We have developed a model of fibrinolysis which predicts the diffusion and permeation of two chain tPA or urokinase (UPA), plasminogen (plg; glu 1 -plg or lys 77 -plg), and plasmin (plm) through fibrin clots as they undergo lysis under any $\triangle \mathrm{P} / \mathrm{L}(0$ to $60 \mathrm{mmHg} / \mathrm{cm}$-clot $)$. This simulation takes into account the kinetics of reversible fibrin adsorption, irreversible reactions in the plasma phase and on the fibrin. The model, which has no adjustable parameters, evaluates: (i) the time-evolving clot structure/binding site stoichiometry, and (ii) lysis fronts moving across clots for any fibrin structure (fine or coarse gels), fibrin density, and therapeutic regimes of uPA and/or tPA (bolus, constant dose, or mixed therapies). From simulations, we predict that:

1) Partitioning of tPA between the plasma phase and the fibrin phase is not at local equilibrium under conditions of fluid permeation across the clot. These nonequilibrium conditions tend to front load the clot with $\mathrm{PA}$

2) The $\Delta P / L$ controls the velocity of a lysis front. With inner clot permeation (Pe -50 to 500), significant clot lysis occurs in under $45 \mathrm{~min}$. However, in the absence of permeation, the model predicts that lysis proceeds at the rate of diffusion and requires many hours to achieve significant lysis. Small amounts of urokinase $(0.1$ to $1 \mu \mathrm{M})$ in conjunction with a tPA $(1 \mu \mathrm{M}) 5$-minute bolus are potent lytic cocktails due to the movement of urokinase with the permeation front, especially at $\mathrm{Pe}>50$. An in vitro perfusion system was used for testing the predictions of the model. In agreement with the computer simulation, a pressure drop across a fibrin clot to drive fluid permeation allows for lysis to occur over 10 fold faster than by diffusion-mediated transport alone.

231

A STUDY OF MICROSCALE FLUID FLOW IN TOTAL ARTERIAL OCCLUSIONS. E.T Ozawa', ${ }^{13}$, R.D. Kamm '3, M. Johnson', J. E. Brush' ${ }^{2}$, and J. Kalan'2. Department of Mechanical Engineering, M.I.T., Cambridge, MA 02139'; Cardiology Section, University Hospital, Boston MA 021182; and the Harvard-M.I.T. Division of Health Sciences and Technology ${ }^{3}$

The use of a catheter to remove or reopen thrombotic occlusions present in arteries is common and has made bypass surgery unnecessary in many cases. The procedure, known as percutaneous transluminal angioplasty (PTA), typically makes use of a balloon-tipped catheter to displace the occlusive material, thereby re-opening the vessel lumen. The flow properties of to displace the occlusive material, thereby re-opening the vessel lumen. The flow properties of
fluid through a chronic total occlusion were studied to improve methods of introducing fluid through a chronic total occlusion were studied to improve methods of introducing thrombolytic enzymes for the purpose of facilitating guide wire penetration during angioplasty. Chronic total occiusions were induced in male New Zealand rabbits by surgical constriction of the left intemal iliac artery. After 8 weeks, the arteries containing the mature occlusions were removed so that a variety of flow measurements could be performed. Baseline measurements of axial flow conductivity at constant pressure through the total occlusion in 22 specimens were followed by a variety of protocols which included 1) treatment with testicular hyaluronidase $(n=5), 2)$ perfusion with an Evans Blue solution $(n=8), 3)$ oscillatory pressure perfusion ( $n=6$,
near physiologic, at $30 \mathrm{~mm} \mathrm{Hg}$ and $100 \mathrm{~mm} \mathrm{Hg}$, and 4) perfusion at several different satic pressure heads $(n=6)$

The experimental results indicate that a possible path of flow past the occlusion is the annular region between the intact internal elastic membrane and the occlusive plug. This hypothesis is further supported by a mathematical model in which data from test (4) ${ }^{*}$ were used to predict the elastic properties of the artery wall, which was assumed to expand away from the to predict the elastic properties of the artery wall, which was assumed to expand away from the patbologic lesion opening an annular channel. Our experimental results were found to be in
approximate agreement with published measurements of arterial wall compliance. These approximate agreement with published measurements of arterial wall compliance. These findings may be relevant to the clinical setting since proteolytic enzymes could take advantage
of this dissecting mechanism to "core" out the occlusive lesion, thus facilitating its removal.

of this dissecting mechanism to "core" out the occlusive lesion, thus facilitating its removal.
Support from the National Science Foundation and the Division of Health Sciences and Technology at M.I.T. for E.T. Ozawa are gratefully acknowledged. 
232

EFFECT OF FLUID SHEAR ON THROMBUS FORMATION AND PLATELET FRAGMENTATION IN HEMODIALYZER

M. Kapadvanjwala, W. Jy, and M.K. Dewanjee

Departments of Biomedical Engineering, Hematology and Radiology, University of Miami, FL.

The effect of pulsatile blood flow on platelet thrombogenecity and platelet fragmentation (PF) in hollow-fiber hemodialyzer (HFD) was quantified with In-111 labeled platelets; 150 ml of blood was collected from Beagle dogs, Yorkshire pigs and human volunteers (nonsmoker). Platelets were labeled with In-111 tropolone $(300 \mu \mathrm{Ci})$ and sham-dialysis (SHD) was performed at $37^{\circ} \mathrm{C}$, with flow rates of $150-, 250-, 500$ - and $950 \mathrm{ml} / \mathrm{min}$. for three hours; after SHD, the washed HD-radioactivity was measured with ionization chamber. Platelet microparticles (PMP) was measured by flow-cytometry with GP IIb-llla murine monoclonal antibody. Procoagulant activity of PMP was measured by Russell Viper Venom test. Platelet deposition decreased significantly for 3 species at higher flow. Adherent platelet thrombus decreased from $(8.2 \pm 3.4)$ to $(3.1 \pm 1.0)$ with human blood as flow rate increased from 150 to $950 \mathrm{ml} / \mathrm{min}$.; platelet thrombus level also decreased significantly from $(20.3 \pm 6.2)$ to $(4.5 \pm 1.9)$ with canine platelets. Higher values were obtained for canine than human and porcine platelets. Platelet fragmentation increased with time and flow rate from 2.1-2.2\% to 10.2-11.3\% (human blood). Procoagulation time for PMP decreased from 61 $\mathrm{sec}$. to $19 \mathrm{sec}$. with time (normal value $52-65 \mathrm{sec}$.). Increase in platelet fragmentation provides more catalytic surface for coagulation and thus reduces the procoagulation time. The study of adherent thrombus, platelet fragmentation and procoagulation activity identified an important flow region for reduced thrombogenicity and acceptable fragmentation encouraging extracorporeal circulation at higher blood flow.

233

EFFECT OF STERICALLY-STABILIZED LIPOSOME-ENCAPSULATED HEMOGLOBIN AND SHEAR FLOW ON ACTIVATION OF COAGULATION AND THROMBOSIS

J. Jato, S. Zheng and R. Beissinger, Illinois Institute of 'Technology, Chicago, IL. 60616 and V. Shankey and J. Fareed, Loyola University Medical Center, Maywood, IL.

Recent work conducted in our laboratories on a hemoglobin-based red blood cell substitute has demonstrated that administration of liposome-encapsulated hemoglobin (LEH) in rats was efficacious. Also it was found that sterically-stabilized LEH was significantly less immunotoxic than conventional LEH. The potential of these two types of formulations for activation of coagulation and platelets following interaction of either hirudin-anticoagulated whole blood or platelet rich plasma (PRP) under controlled shear flow conditions is being investigated in this study. Intially, conventional LEH containing phosphatidylglycerol (PG) and sterically-stabilized LEH in which PG is replaced with polyethylene glycol-phosphatidylethanolamine (PEG-PE) are compared for their effects on platelet activation. Activation is measured using flow cytometric analysis of CD-62 monoclonal antibody (GMP-140) expression on CD-61 monoclonal antibody (GP IIb/IIIa) positive platelets. These studies are being carried out in a rotational flow system, i.e. a cone-and-plate viscometer, to assess shear flow effects on bulk interactions between platelets and LEH. Activation of enzymatic coagulation reactions is being investigated under controlled shear flow conditions with either whole blood or PRP flowing through a capillary system with LEH coated on the capillary wall. Results obtained in these studies thus far indicate that the sterically-stabilized LEH do not activate platelets.

\section{4}

FLOW CYTOMETRIC ANALYSIS OF SHEAR INDUCED PLATELET ACTIVATION

N.B. Purvis, Jr. and T.D. Giorgio

Department of Chemical Engineering, Vanderbilt Univ, Nasbville, TN

Past studies of shear induced platelet activation (SIPAC) have been limited to assays of averaged platelet population changes. Recent studies of chemically induced platelet activation have demonstrated that $15 \%$ to $30 \%$ of the platelet population does not participate in activation and aggregation. Interpretation of assays, such as the measurement of intracellular $\mathrm{Ca}^{2+}$ concentration ( $\left.\left(\mathrm{Ca}{ }^{2+}\right]_{j}\right)$, may be complicated since the results average both activated and non-activated platelets. Flow cytometry provides the means to distinguish between activated and non-activated platelets. Furthermore, flow cytometric measurements are made on individual platelets allowing greater sensitivity to changes that might otherwise be undetectable

Platet rich pasma (PRP) was exposed to a contled

Platelet rich plasma (PRP) was exposed to a controlled, uniform shear stress of 32 dynes $/ \mathrm{cm}^{2}$ in a cone and plate viscometer for 60 seconds. Sheared platelet samples were immediately fixed in $1 \%$ paraformaldehyde and subsequently labeled with the monoclonal antibodies (MoAbs), which recognize specific epitopes on platelet membrane proteins and receptors. Control and phorbol myristate acetate (PMA) treated platelets were identically fixed and labeled. The platelet samples were analyzed in a FACScan flow cytometer gating only positive platelet events. Fluorescence data was converted to the number of bound MoAb molecules per event and surface area normalized to decouple simultaneous changes in platelet size and receptor expression. CD62 binding density, a MoAb specific for $\alpha$ granule membrane protein GMP-140, was determined to be $75 \pm 13$ molecules per $j \mathrm{~mm}^{2}$ following a 1 minute exposure to 32 dynes $/ \mathrm{cm}^{2}$. CD62 binding densities of $59 \pm 16$ and $313 \pm 67$ molecules per $\mu \mathrm{m}^{2}$ were measured for control and PMA treated platelet samples, respectively, which corresponds to an average percent $\alpha$ granule release of $6.0 \%$. SIPAC induced binding of fibrinogen and von Willebrand Factor as well as changes in GP Ilb/Mla expression were also measured by flow cytometry. Additional assays were performed to monitor $\left[\mathrm{Ca}^{2+}\right]_{\mathrm{i}}$, dense granule release, lysis, and aggregation.
235

FLOW CYTOMETRIC STUDIES OF PLATELETS FROM NORMAL SUBJECTS AND STROKE PATIENTS SURJECTED TO SHEAR STRESS

K. Konstantopoulos ${ }^{1}$, J.C. Grotta ${ }^{2}$, M.M. Udden ${ }^{3}$, E.I. Banez ${ }^{3}$, K.K. Wu ${ }^{2}$, and J.D. Hellums ${ }^{1}$ $\mathrm{Cox}$ Laboratory for Biomedical Engineering, Rice Universityl; University of Texas Health Science Center ${ }^{2}$; Baylor College of Medicine ${ }^{3}$ The objective of this work was to evaluate quantitatively the effects of flow on platelet reactions using a flow cytometric technique. Whole blood specimens from normal subjects and
atherothrombotic stroke patients (up to 72 hours post stroke) were exposed to well-defined. atherothrombotic stroke patients (up to 72 hours post stroke) were exposed to well-defined, laminar shear stress in a cone-and-plate viscometer in the absence of added agonists. Specimens
were fixed with formaldehyde, and then incubated with two fluorochrome-conjugated monoclonal were fixed with formaldehyde, and then incubated with two fluorochrome-conjugated monoclonal
antibodies. Antibody $6 \mathrm{D} 1$, specific for platelet membrane glycoprotein Ib (GPIb), was used to antibodies. Antibody $6 \mathrm{D} 1$, specific for platelet membrane glycoprotein Ib (GPIb), was used to
identify, and enumerate platelets and platelet aggregates, on the basis of their characteristic forwand-angle light scattering and FITC fluorescence profiles. Antibody PAC1, specific for the activated form of GPIIb-IIL, was used to measure platelet activation. Blood specimens were then analyzed in a FACScan flow cytometer. The results on normal blood showed platelet activation and aggregation increase with the shear stress. The stroke patients blood was found to exhibit much bigher than nomal levels of platelet activation prior to exposure to shear stress: $11 \%$ \pm 1.7 (meantS.E.M) activated versus $6 \% \pm 0.5$ for nommals. Furthemore, the stroke patients \pm 1.7 (mean \pm S.E.M) activated versus $6 \% \pm 0.5$ for normals. Furthermore, the stroke patients
blood was much more susceptible to shear-induced platelet activation and aggregation. At a blood was much more susceptible to shear-induced platelet activation and aggregation. At a
stress level of 105 dynes $/ \mathrm{cm}^{2}$ stroke patients platelets formed $3000 \pm 300$ large aggregates per $\mu$ ("large" defined to be larger than leukocytes). In contrast, nommal specimens formed only $400 \pm 80$ large aggregates per $\mu$, The results show that shear stresses of the levels which occur in arteries partially occluded by atherosclerosis or vascular spasm strongly activate and aggregate platelets. and this response is much more pronounced in stroke patients.

\section{6}

PLATELET AGGREGATION AND SURFACE ADHESION IN PULSATILE SHEAR FLOW: EFFECTS OF RED BLOOD CELLS

J. H. Joist*, J. A. Bauman*, S. P. Sutera ${ }^{\dagger}$

${ }^{*}$ St. Louis University, †Washington University, St. Louis, MO

Red blood cells (RBC) are known to play an important role in the arrest of bleeding following small vessel injury. They also support platelet adhesion to endothelial cell matrix and artificial surfaces as well as platelet aggregation in shear flow in different in vitro flow systems. Experiments were designed to examine the effects of RBC on shear-induced platelet aggregation (SIPAG) and platelet adhesion (SIPAD). Suspensions of human platelets labeled with Mepacrine and suspended in citrated plasma were exposed to pulsatile shear stress of varying amplitude $\left(25-100 \mathrm{dyn} / \mathrm{cm}^{2}\right)$ in a cone-plate viscometer in the presence or absence of intact RBC or glutaraldehyde-fixed (GTA) RBC for 120130 seconds. SIPAG was expressed as percent loss of single platelets. SIPAD was assessed in terms of Mepacrine-related, fluorescent materials remaining on glass disks inserted in the bottom plate of the viscometer after washing with EDTA-saline. Intact RBC were twice as effective as GTA-RBC (which are depleted of ADP) in potentiating SIPAG at all shear stress levels. Potentiation of SIPAD by intact RBC was markedly less than that observed with GTA-RBC at stresses above $50 \mathrm{dyn} / \mathrm{cm}^{2}$. These and additional findings indicate that RBC potentiate SIPAG by both physical and humoral (ADP) mechanisms but support SIPAD largely if not solely by enhancement of platelet transport from the bulk flow to the bounding surfaces.

\section{7}

EFFECTS OF LOW FLUID SHEAR RATE ON TISSUE FACTOR ACTIVITY IN CULTURED HUMAN MONOCYTES Steven $M$. Peckham, Lee J. Carmack, Yale Nemerson*, and Vincent T. Turitto (Department of Biomedical Engineering, Memphis State University, Memphis, TN 38152, and *Mount Sinai Medical Center, New York, NY 10029)

The effects of fluid shear on the coagulative behavior of cultured macrophages derived from human peripheral blood monocytes (PBM) have been investigated. Using a parallel plate perfusion chamber cells cultured 7 to 14 days on Thermanox slides were exposed to wall shear rates of $6,12,24$, or $48 \mathrm{~s}^{-1}$ for up to 2 hours in $0.01 \mathrm{M}$ Hepes buffer containing $150 \mathrm{nM}$ factor $X(\mathrm{FX})$ and $10 \mathrm{nM}$ factor VIIa (FVIIa). The concentration of FXa exiting the chamber was determined by chromogenic assay and found to increase with increasing time and decrease with increasing wall shear rate. Omitting FVIla resulted in no FXa production over a 1 hour perfusion. Prior to culture, peripheral blood monocytes exhibited no tissue factor (TF) activity, as measured by this assay system. Thus, the culture of PBM to nearly confluent macrophages resulted in cells exhibiting TF activity; the extent of this activity depended on the local shear conditions in a manner quantitatively predicted by theoretical considerations of substrate transport to the cells. 
238

COMPARATIVE HEMORHEOLOGY OF ATHLETIC AND SEDENTARY MAMMALIAN SPECIES

A.S. Popel and M.V. Kameneva

Dept. of Biomedical Engineering, Johns Hopkins University, Baltimore, MD 21205 and Dept. of Surgery, University of Pittsburgh, Pittsburgh, PA 15213

Blood viscosity was measured with a Couette theometer in the range of shear rates 0.277 to 128.5 $\mathrm{s}^{-1}$ and with a capillary viscometer at $500 \mathrm{~s}^{-1}$ for samples of ovine, canine, antelope (pronghorn antelope, Antilocapra americana) and human blood. In addition, erythrocyte sedimentation rate (ESR) was measured using the Wintrobe method. Among these mammalian species, sheep is a sedentary species, and dog and antelope can be characterized as athletic, based on mass-specific maximum oxygen consumption, $\dot{\mathrm{V}}_{2 \max }$, a parameter used to characterize animals athletic ability. Humans are a special case in such classification, because of bipedal gait vs quadrupedal animals. Our data stow that the degree of red blood cell aggregation, as characterized by the viscosity at low shear rate and ESR, is higher in the athletic antelope and dog, as compared to sedentary strep. Comparative analysis can also be done in terms of parameters in the Quemada theological model. Analysis of data by others corroborate this finding: goat (sedentary) has similar low aggregation capacity as sheep, athletic horse has a high aggregation capacity, whereas in sedentary cow red blood cell aggregation is virtually absent. Hemodynamic analysis of resistance changes and pressure distribution throughout the microcirculation in skeletal muscle between resting conditions and strenuous exercise shows that a substantial increase in blood viscosity in the postcapillary circulation would serve to prevent capillary pressure from falling to very low values in transition from exercise to rest in athletic species, in spite of significant increases in precapillary resistance. We hypothesize that during evolution athletic species developed the capacity for red blood cell aggregation as a means of regulating postcapillary hemodynamic resistance that serves to maintain levels of capillary hydraulic pressure necessary for normal fluid balance. Supported in part by NIH grants HL 17421 and $\mathrm{HL} 18292$.

239

SURFACE SHEAR VISCOSITY OF RED CELL MEMBRANE FROM RHEOSCOPIC OBSERVATIONS

A. Millous, S. P. Sutera, G. I. Zahalak

Washington University, St. Louis, MO

Measurements of the deformation and membrane rotational frequency of individual red blood cells (RBC) steadily tank-treading in a Rheoscope are used to calculate the surface shear viscosity $\left(\eta_{m}\right)$ of the membrane. The calculation employs previously developed algorithms based on a mechanical energy balance plus an assumed membrane velocity field. The method yields either a surfaceaveraged value of $\eta_{m}$ corresponding to a limited interval of membrane shear rate or a constitutive relationship for $\eta_{\mathrm{m}}$ as a function of the second invariant of surface strain rate. In the latter representation of $\eta_{\mathbf{m}}$ is found to decrease with increasing strain rate (shear-thinning behavior). The range of $\eta_{\mathrm{m}}$ determined in this manner tends to be substantially below those deduced from observations of the transient recovery of RBC deformed by means of micropipette manipulation. The discrepancy raises questions about certain assumptions inherent in the micropipette recovery approach.

240

FLOW INDUCED MICROSTRUCTURE AND YIELD STRESS OF HUMAN BLOOD

George B. Thurston

Rheology Laboratory, Department of Mechanical Engineering

The University of Texas at Austin, Austin, TX 78712

The viscoelastic properties of human blood change with flow conditions, and the balance between elastic energy stored in the blood microstructure and the rate of dissipation of energy is altered [1]. While at low rates of flow the blood structure is isotropic on a macroscopic scale, it become highly anisotropic at high rates of flow. Under oscillatory flow at controlled frequency and amplitude (in the range of flow conditions in vivo), the transition in structure is observed to be progressive with increasing amplitude. Near unit strain a dramatic change occurs which limits the increase in elastic energy storage per unit strain. This critical point is identified by a maximum in elastic stress, marking the onset of formation of cell layers separated by plasma layers [2]. This elastic yield stress is identified both in measurements of the viscoelastic stress-strain relation and in the transmission of light through the blood [3]. Because the red cell dominates the viscoelasticity of blood, the structural yield properties and the transition to layered flow are dependent on both the dynamic deformability and the aggregation tendency of the cells. Examples are given which show how these factors affect the elastic yield stress. In order to understand blood flow in vivo and the shear force on vessel walls, the effects of layered flow must be considered.

[1] G. B. Thurston, Biophysical J. 12, 1205-1217 (1973).

[2] G. B. Thurston, Biorheology 26, 199-214 (1989).

[3] G. B. Thurston, Biorheology 27, 685-700 (1990).
241

\section{ULTRASOUND METHODS FOR INVESTIGATING BLOOD RHEOLOGY}

R.S.C. Cobbold, R.E.N. Shehada, L.Y.L. Mo and P.A.J. Bascom Institute of Biomedical Engineering,

University of Toronto, Toronto M5S 1A4, Canada.

Although a great deal of work has been devoted to studying the non-Newtonian characteristics of blood in small diameter vessels, limitations of current measurement techniques have not enabled detailed studies to be conducted on blood flowing in large diameter $(>1 \mathrm{~cm})$ tubes. It is our purpose to describe some recently developed ultrasound methods that enable quantitative information to be obtained from large diameter tubes.

A linear array $7 \mathrm{MHz}$ B-mode imaging system was used to measure blood echogenicity and velocity profiles simultaneously as they developed with axial distance for a steady flow of porcine whole blood $(\mathrm{Hct}=28 \%)$ in a long, large diameter $(2.54 \mathrm{~cm})$ tube. Velocity profiles were measured using cross correlation between successive digitized images with a known time separation, and from these shear rate profiles were calculated. Mean echogenicity profiles were also determined from the digitized images for selected locations along the flow axis. It was found that over a range of low shear rates, the echogenicity is enhanced similar to the previously reported influence on aggregation. The transient effects of abrupt flow stoppage were also studied and it will be shown how these results can provide useful insights on aggregation kinetics.

242

INFLUENCE OF NON-NEWTONIAN BEHAVIOR OF BLOOD ON FLOW IN AN ELASTIC ARTERY MODEL

A. Dutta and J.M. Tarbell

Department of Chemical Enginecring

The Pennsylvania State University

University Park, PA 16802

Two different non-Newtonian models for blood, one a simple power law model exhibiting shear thinning viscosity and the other a generalizod Maxwell model displaying both shear thinning viscosity and oscillatory flow viscoelasticity, were used along with a Newtonian model to simulate fully developed oscillatory flow of blood in rigid and elastic straight arteries.

When the spring elements were removed from the viscoelastic model resulting in a purely viscous shear thinning fluid, predictions of flow rate and wall shear stress (WSS) for a fixed pressure gradient waveform were unaltered, suggesting that elasticity of blood does not significantly influence blood flow under arterial flow conditions. Simulations using a power law model with a high shear rate Newtonian cutoff viscosity and a fixed pressure gradient waveform as input in both rigid and elastic tubes indicated that the mean WSS is quite insensitive to the fluid rheology but strongly dependent on the phase angle between the elastic wall motion and flow waveforms. The effect of elastic wall motion on WSS could be greatly reduced by matching the flow rate of the elastic and rigid models rather than the pressure gradient. 
243

OXYGEN DIFFUSION IN SICKLE CELL BLOOD

Charles Y. Liu, ${ }^{1}$ M. M. Udden, ${ }^{2}$ and J. David Hellums'

Cox Laboratory for Biomedical Engineering, Rice University, ${ }_{1}^{1}$ Houston, TX, 77251-1892;

Polymerization of sickle cell hemoglobin accompanies deoxygenation and is known to be the primary event in sickle cell crisis. The present study used a diffusion cell to examine the effect of HbS polymerization on the oxygen effective diffusivity in sickle erythrocytes. Permeability experiments were conducted on samples of normal hemoglobin $(\mathrm{HbA})$ solution, packed normal erythrocytes, and packed SS erythrocytes which contained varying amounts of fetal and $\mathrm{C}$ hemoglobin. In increasing $\mathrm{PO}_{2}$ experiments, the samples were fully oxygen saturated after a very brief transient. In decreasing $\mathrm{PO}_{2}$ experiments, the average oxygen tension decreased progressively over the time course of the experiment.

At full oxygen saturation, the oxygen effective diffusivity in the packed sickle erythrocyte samples was not significantly different from that in normal erythrocytes, and was in agreement with prior worker's measurements of unfacilitated oxygen diffusion. The effective oxygen diffusivities measured in the decreasing $\mathrm{FO}_{2}$ experiments on packed sickle erythrocytes were significantly different from those in normal erythrocytes. As the average oxygen tension decreased, the effective diffusivity in packed normal erythrocytes increased progressively to a maximum of $40 \%$ over its unfacilitated value, and then decreased. In contrast, in $\mathrm{SS}$ erythrocytes as $\mathrm{PO}_{2}$ decreased, the effective diffusivity increased only slightly, and then decreased dramatically. The results of decreasing $\mathrm{PO}_{2}$ experiments on sickle erythrocytes containing significant amounts of other hemoglobins ( $\mathrm{HbF}, \mathrm{HbC}$ ) were different from those of both the normal erythrocytes and sickle erythrocytes with dominant $\mathrm{HbS}$ fraction, showing the effect of hemoglobin composition on effective diffusivity. These results demonstrate a dramatic effect of $\mathrm{HbS}$ polymerization on the resistance to oxygen transport in sickle erythrocytes.

244

RED GELL DEFORMABILITY ANO ADHESION TO ENDOTHELIUM AS DETERMINANTS OF VASO-OCCLUSIVE EPISODES IN SICKLE CELL DISEASE

H. H. LIPOWSKY

Bioengineering Program, Penn State University, University Park, PA 16802

The onset of vaso-occlusive crisis in the sickle cell disease has been attributed to either diminished red blood cell (rbc) deformability, attendant to deoxygenation and polymerization of HbSS hemoglobin, or adhesion of rbcs to the endothelium (EC). To examine the role of rbc deformability in promoting microvascular stasis, measurements of the transit time of rbcs ( $\Pi_{r b c}$ ) hardened by glutaraldehyde were made in the microvasculature of cremaster muscle (rat). RBC deformability was evaluated by filtration, in terms of the ratio of resistance to flow through pores of a Nuclepore filter, to that of suspending medium alone, i.e. $\beta=R_{r b d} / R_{p o r e-}$ It was found that $T T_{r b c} / T_{p 1}$ (normalized with respect to plasma transit time) was invariant with $\beta$ for $2.5<\beta \leq 10$ due to shunting of less deformable rbcs through pathways of lesser resistance, and rose significantly with $\beta$ for $\beta>10$. In comparison, values for well oxygenated HbSS rbcs are on the order of $\beta \approx 8$ (Schmalzer, E. A. et al. J. Lab. Clin. Med. 113, 724-734, 1989), and upon deoxygenation, large decreases in rbe deformability preclude a determination of $\beta$. Thus, the sickle cell patient appears to be functioning near a critical level of deformability, beyond which further increases in $\beta$ may lead to microvascular stasis. To evaluate the potential for rbc-EC adhesion to exacerbate a low flow state, measurements of rbc velocity were made in nailfold capilfaries of patients with sickle cell disease while reducing flow velocities by occlusion of the digital artery with a pressure cuff. It was found that creeping velocities of rbcs persisted at calculated wall shear stresses on the order of $0.25-0.5 \mathrm{dyn} / \mathrm{cm}^{2}$ and that the inability to dispupt aggregates of rbcs appeared to result in capillary stasis in the nailfold. Thus rbc-EC adhesion may contribute less to vaso-occlusion than rbc deformability and aggregation.

Supported in part by NIH Grants HL-28381 and HL-39286.

245

AL.TERATION OF SICKLE CELL ADHESION TO ENDOTHELIAL CELLS BY ATA.

M. Natarajan ${ }^{1}$, M.M. Udden ${ }^{2}$, D. Sears ${ }^{2}$, J. Moake ${ }^{3}$ and L.V. McIntire ${ }^{1}$

Cox Laboratory for Biomedical Engineering, Rice University. Houston, TX ${ }^{1}$

Medical Hematology Section, The Methodist Hospital, Houston, $\mathrm{TX}^{2}$

Department of Medicine, Baylor College of Medicine, Houston, TX $^{3}$

Abnormal adherence of sickled erythrocytes to human endothelial cells correlates with the clinical severity of the sickle cell anemia disease. Earlier work by Wick et al. (Am. J. Hematol., 42:284-292 (1993)) showed that human vWF promotes sickle erythrocyte adhesion to endochelial cells. Endothelial cells secrete ultra-large vWF that is thought to bind to GPIblike receptors on erythrocytes. Our objective is to test the effects of aurin tricarboxylic acid, a polycarboxylic, polyphenolic compound that has been shown to interact with von Willebrand factor and inhibit its binding to platelet GPIb receptors (Blood, 81(5): 1263-1276 (1993)), on the adhesion of normal and sickled red blood cells to endothelial cells.

A parallel plate flow chamber and a phase contrast microscope with a computerized stage were used to evaluate the effects of ATA on this adhesion. Sickled reticulocytes were separated from whole blood, incubated with endothelial cell supematant (EC-supematant) for one hour at $37^{\circ} \mathrm{C}$ and perfused over a monolayer of endothelial cells. ATA was incubated with the erythrocyte-EC supermatant suspension for five minutes prior to its perfusion over the endothelial cell monolayer. The number of adherent cells were counted in sixteen random fields ranging over the entire flow chamber. Reticulocytes from donors with non-sickle hemoglobin served as controls for these experiments.

Preliminary results demonstrate concentration and incubation time effects of ATA on the athesion of red cells to endothelial cells. The effect of a recombinant vWF fragment from the GPIb binding domain on the adhesion of normal and sickled cells to endochelial cells will also be investigated.
246

RECEPTOR-MEDIATED ADHERENCE OF SICKLE ERYTHROCYTES TO VASCULAR ENDOTHELIUM TM Wick and JR Eckman, School of Chemical Engineering, Georgia Institute of Technology, Atlanta, GA and Division of Hematology/Oncology, Depe
Medicine, Emory University School of Medicine, Atlanta, Georgia.

Occlusion of microvessels by sickle erythrocytes results in the episodic pain events characteristic of sickle cell anemia. Microvascular occlusion is likely initiated or accelerated by adherence of sickle erythrocytes to the vascular endothelium. It is hypothesized that inhibition or reversal of adherence will reduce the frequency and severity of sickle cell pain events and lessen the accompanying organ damage. In vitro, adherence is dependent upon sickle red cell membrane alterations, sickle plasma factors, endothelial cell phenotype, endothelial cell activation state, and local hemodynamic conditions. Our group and others have recently described several receptor-mediated pathways of sickle cell adherence to large vessel and microvascular endothelium. Both integrin and non-integrin receptors on sickle enthrocyles and endothelium participate in adherence. Adhesion is greater to cytokine activated endothelium. Our group has recently reported that mechanism(s) of sickle cell adherence to capillary and post-capillary venule mechanism(s) of sickle cell adherence to capillary and post-capillary venule
endothelium are different (Brittain, et al. J Lab Clin Med 1992;19:538). These endothelium are different (Brittain, et al. J Lab Clin Med 1992;19:538). These observations indicated that adherence of sickle erythrocytes to microvascular endothelium is elaborate and heterogeneous. In this report, the evolution of vaso-
occlusion theory will be presented and the role of adherence in the pathophysiology of vaso-occlusion will be discussed. Potential therapeutic targets based on the adherence data will also be discussed.

247

TISSUE PERFUSION IN MICE CARRYING THE GENE FOR HUMAN SICKLE HEMOGLOBIN

Ingrid H. Sarelius. Patricia A. Titus, and Giles R. Cokelet.

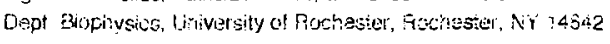

Seveial trarisyenic mouse models for sickle cell disease have been reported. We have established a colony of mice carrying the HS1-5 $\mathrm{H}^{5}$ gene; $58 \%$ of these animals express human sickle hemoglobin ( $\mathrm{HbS}$ ) as $40 \%$ of total hemoglobin. This experimental model provides an opportunity to directly explore microvascular function in circulations that have adapted to the disease process (e.g. as a result of anemia or from repeated perfusion impairment) by remodeiling or by changes in microcirculatory control mechanisms. Mice with the gene (POS) have higher blood density, higher spleen weight, and lower hematocrit by 14 weeks of age than non gene-carrying matched controls (NEG). Cremaster preparations in anesthetised mice $(7 \mathrm{mg} / 100 \mathrm{~g}$, i.p.) were used to compare capillarity, capillany cell flow variables and anteriolar responses. POS mice had fewer capillaries than NEG mice but almost all of these capillaries were perfused at rest, whereas NEG animals had a significant capillary reserve. Cell flow capacity was higher in POS mice ( $90.6 \pm 15.3$ (S.E.) vs $70.9 \pm 6$ ceils/sec in NEG); also, in POS, capillary cell content was higher $(25.5 \pm 1.8$ vs $20.1 \pm 1.2$ cells $/ 100 \mu \mathrm{m})$ while cell velocity was lower $(192 \pm 12$ vs $223 \pm 12 \mu \mathrm{m} / \mathrm{sec}$ ) and perfused capillary segments were shorter $(303 \pm 31$ vs $387 \pm 33 \mu \mathrm{m})$. These data show that there are differences in tissue blood flow characteristics in $\mathrm{HbS}$ mice which may reflect adaptational responses to changes in blood cell characteristics or oxygenation. 
LEUKOCYTE CAPILLARY OBSTRUCTION IN THE PERIPHERAL CIRCULATION.

G. W. Schmid-Schönbein

Institute for Biomedical Engineering, University of California, San Diego, La Jolla, California 92093-0412

Since the discovery of leukocyte entrapment in the capillary network of skeletal muscle during hemorrhagic shock, this phenomenon has been reported in the capillaries of the coronary, cerebral, hepatic, renal, pancreatic, dermal, mesenteric, and retinal circulation. Leukocytes are prone to such entrapment due to their large size, compared with the lumen dimensions of capillaries, their high cytoplasmic stiffness, and their ability to adhere to the endothelium. There are two mechanisms by which leukocytes may become entrapped in capillaries: by reduction of the microvascular perfusion pressure or after stiffening of their cell cytoplasm. Cell stiffening occurs after pseudopod formation and may be accompanied by an elevation of cell membrane adhesion via expression of membrane adhesion glycoproteins. In low flow conditions, reduction of the microvascular perfusion pressure may cause relaxation of the capillary lumen and protrusion of the endothelial nuclei, further facilitating leukocyte entrapment. The duration of leukocyte entrapment in capillaries depends on the perfusion pressure history, the degree of adhesion to the endothelium and membrane contact area, and may involve a wide range of times from temporary plugging at capillary entrances to permanent obstruction. Obstruction of capillaries after restoration of the perfusion pressure may lead to a capillary no-reflow phenomenon which is one of the complications that accompanies reperfusion after ischemia or shock. Supported by USPHS grant HL 10881 and HL 43026.

250

SEQUESTRATION OF NEUTROPHILS INDUCED BY INTRAVASCULAR COMPLEMENT PROTEIN FRAGMENTS. C.M. Doerschuk, H. Inano, N.A Doyle and W.M. Quinlan. H.B. Wells Center for Pediatric Research, Section of Pulmonology, Department of Pediatrics, Indiana University.

Infusion of zymosan-activated plasma, a source of active complement-derived proteins, induced a rapid decrease in the circulating neutrophil count due to sequestration of neutrophils within all organs, particularly the lung. In the pulmonary microvasculature, the major site of neutrophil sequestration was the capillary bed, preferentially the junctions of the capillary segments. Recent studies have focussed on the roles of neutrophil deformability and neutrophil-endothelial cell adhesion in sequestration. Neutrophil deformability, determined by measuring the pressure required to pass neutrophils through a filter with $5 \mu \mathrm{m}$ pores, decreased within 1 minute after exposure to complement fragments. This decrease was prevented by pretreatment with cytochalasin $\mathbf{B}$, an inhibitor of $\mathbf{f}$-actin polymerization, but not colchicine, an inhibitor of microtubule reassembly. Studies focussed on the role of adhesion molecules showed that chymotrypsin-induced depletion of L-selectin or inhibition of $\mathrm{CD} 11 / \mathrm{CD} 18$ did not prevent complement fragment-induced sequestration. However, CD11/CD18 was required for the neutrophils to remain sequestered for $>4-6$ minutes, despite continuous infusion of mediators. These studies suggest that neutrophil sequestration is a sequential process whereby an initial decrease in deformability and stiffening of neutrophils prevents them from passing through the pulmonary capillary bed followed by activation of adhesion molecules that is required for neutrophils to remain sequestered.

Supported by PHS HL 48160 \& a Career Investigator Award from Am. Lung Assoc.

251

NEUTROPHIL TRANSIT THROUGH THE PULMONARY MICROCIRCULATION

B.R. WIGGS*

MIT Fluid Mechanics Lab, Dept. Mechanical Engineering, Cambridge, MA

Several experiments have shown that neutrophils (PMN) are delayed with respect to red blood cells (RBC) as they pass thorugh the microvessels of the lung. The purpose of the present study was to develop a stochastic simulation of PMN transit to explore the role of cell deformability in the observed differences in PMN and $\mathrm{RBC}$ transit in the lung. The pulmonary capillary microcirculation was modelled using mesaurements of capillary segment length and diameter, the number of segments in an alveolar wall and the knowledge that PMN pass through an interconnected network of alveolar walls as they travel from arteriole to venule. The time required for PMN to enter the small capillary segments was based on an exponential relationship (Pflugers Arch:430:396, 1985) that used the cell/vessel as the time determinant. The model, when coupled to a large vessel simulation of the arterial and venous networks, predicts RBC transit times similar to those found experimentally but the delay between PMN with respect to RBC was not fully explained by this simple model based only on differences in cell deformability.

*Fellow of the American Lung Association
252

COMPUTER MODELING OF NEUTROPHIL TRAFFIC IN THE PULMONARY CAPILLARIES. W.W. Wagner, Jr., C.C. Hanger, R.L. Capen. Indiana University Medical School, Indianapolis, IN and The Colorado College, Colorado Springs, CO

To simulate neutrophil traffic through the pulmonary capillary bed, we developed a computer model of cells moving through a matrix of interconnected tubes. The matrix size, typically $27 \times 27$, was chosen so that the trans-matrix pathway was 60 segments long, the calculated length of an average pulmonary capillary pathway. Because previous work using in vivo video microscopy had shown that neutrophil movement through the capillary bed involved a combination of rapid movements interrupted by pauses at discrete sites, individual segments in the model were designated as sites that would impede the movement of passing neutrophils. We were particularly curious to determine what percentage of the segments had to be obstructed to impede $50 \%$ of the cells, because we have found that $50 \%$ of fluorescein-labeled neutrophils stopped in subpleural capillaries. Using runs of 10,000 cells for each percentage impediment, we were surprised to learn that obstruction of only $1 \%$ of the segments stopped $50 \%$ of the cells. This relationship held regardless of the matrix design (adjacent triangles, squares, hexagons) or whether the obstructions were placed in the segments or the junctions. When the size of each neutrophil and each capillary segment was chosen from histograms constructed from anatomical measurements (with both vessels and cells being rigid), all cells stopped many times (average 38, minimum 12). These data suggest that deformability plays an essential role in the ability of the neutrophil to transit the pulmonary capillary bed. Supported by Howard Hughes Medical Institute 71108503101 and NIH HL-36033.

253

EFFECTS OF LEUKOCYTE-CAPILLARY PLUGGING AND VENULAR ADHESION ON SKELETAL MUSCLE FLOW RESISTANCE.

T.C. Skalak', M.C. Mazzoni ${ }^{2}$, K.C. Warnke ${ }^{1}$

${ }^{1}$ Dept. of Biomedical Engineering. University of Virginia, Charlottesville, VA 22908, and 2Institute for Biomedical Engineering, Univ, of Califomia, San Diego, La Jolla, CA 92093

Leukocyte-capillary plugging has been suggested to play a role in the impairment of microvascular flow, particularly after leukocyte activation. On the other hand, leukocyte activation also leads to significant venular adhesion, which represents an additional hydrodynamic obstruction that plays a role in the overall microvascular flow impairment. A network model of an arteriolar-venous capillary unit in skeletal muscle was used to evaluate the relative effects of leukocyte-capillary entrance plugging and venular adhesion on overall network resistance. The capillary unit resistance was computed for specified arterio-venous pressure gradient, systemic leukocrit, leukocyte viscosity, and vessel dimensions. The conditions studied for impact of plugging alone were: control, hypoperfusion ( $45 \%$ decrease in pressure gradient), capilary narrowing ( $21 \%$ decrease in diameter), and leukocyte activation ( 10 fold increase in cytoplasmic viscosity). The effect of yenular adhesion on flow resistance was studied by reducing all venular segment diameters by $5 \mu \mathrm{m}$. In control the network resistance attributable to leukocyte capillary plugging was $1.8 \%$, and hypoperfusion produced no significant change. Capillary narrowing elevated the value slightly to $3.7 \%$, and leukocyte activation elevated it to $5.5 \%$. For conditions simulating hemorrhagic shock with hypoperfusion and capillary narrowing, the increase due to plugging is $2.1 \%$ for unactivated leukocytes and $12 \%$ for activated leukocytes. Under these conditions, the additional resistance change due to venular adhesion is $6 \%$. Thus, the relative impact of capillary plugging and venular adhesion on network resistance depends strongly on the activation state of the leukocytes. If the cells are activated with elevated viscosities, then plugging has greater impact than venular adhesion, whereas the converse is true for unactivated cells. Supported by NIH HL-39680.

254

LEUKOCYTE-CAPILLARY PLUGGING AND ADHESION TO ENDOTHELIUM AS DETERMINANTS OF MICROVASCULAR BLOOD FLOW IN THE LOW FLOW STATE H. H. Lipowsky and M. J. Eppihimer

Bioengineering Program, Penn State University, University Park, PA 16802

Obstruction of capillaries by leukocyte (WBC) sequestration has been implicated as a cause of microvascular flow degradation in the low flow state, and WBC adhesion to the endothelium (EC) of postcapillary venules has been shown to result in large increases in the resistance to blood flow. To examine the relative importance of these two mechanisms by which microvascular blood flow may be compromised, capillary plugging by WBCs and WBC-EC adhesion were quantitated in an induced low flow state in the cremaster muscle (hamster). A continuous infusion of sodium nitroprusside was used to reduce Newtonian wall shear rate, $\dot{\psi}$, from a norm of $773 \pm 380$ SD to $416 \pm 220$ SD sec ${ }^{-1}$ in the true capillaries, and from a norm of $760 \pm 450$ SD to $270 \pm 147$ SD sec in postcapillary venules. These reductions in $\hat{\gamma}$ resulted in a twofold rise in the number of WBCs adhering to the venular EC, which progressively increased with time as the low flow state persisted. Using the two-slit photometric technique, estimates of the percentage increase in resistance to flow were made at the capillary level by assuming that the number of patent capillaries, relative to post-capillary collecting venules, was determined by the ratio of the volumetric flow in venules to that obtained in capillaries. In the low flow state, the number of flowing capillaries fell to about $65 \%$ of that in the normal flow state. The increased resistance in postcapillary venules was calculated from in vivo correlations of resistance vs the number of WBCs adhered per $100 \mu \mathrm{m}$ of venule length. Calculations of the percentage increase in resistance to the total throughput of the network in capillary and venular segments, revealed that WBC-EC adhesion in venules contributed to a $20 \%$ greater increase in segmental resistance compared to capillary plugging by WBCs.

Supported in part by NIH Research Grants HL-28381 and HL-39286 
255

ALTERATIONS IN MICROVASCULAR NETWORK PROPERTIES OF RAT CREMASTER MUSCLE DURING THE DEVELOPMENT OF REDUCED RENAL MASS HYPERTENSION. A.S. Greene, D.M. O'Drobinak, P.J. Tonellato, M.J. Rieder, and J.H. Lombard. Medical College of Wisconsin, and Marquette University, Milwaukee, WI 53226

A mathematical model of the microcirculatory network in the rat cremaster muscle was defined based on morphological measurements from histologically prepared tissue whole mounts as well as from observations made in vivo. Measurements of microvessel density, capillary red blood cell ( $R B C$ ) velocity (video cross correlation), arteriolar flow rates, and diameters were performed during physiological salt solution and Adenosine $\left(10^{-4} \mathrm{M}\right)$ superfusion in reduced renal mass (RRM) and Sham rats at control, 3 days and 4 weeks following the onset of hypertension. Experimental results indicated a progressive microvessel rarefaction, but no change in average diameter of first, second, or third order arterioles at either 3 days or 4 weeks. Flow in the remaining third order arterioles of RRM was not significantly increased at 3 days but was slightly elevated by 4 weeks. Capillary RBC velocity was increased in RRM at 4 weeks. Integration of the experimental data into the model revealed an increased capillary flow heterogeneity and the presence of substantial changes in pressure distribution throughout the network of the RRM which was primarily the result of vascular rarefaction. Supported by NIH HL-25987 and AHA 92F-11.

\section{6}

ERYTHROCYTE DISTRIBUTION AT MICROVASCULAR BIFURCATIONS GR Cokelet, JS Gessert, IH Sarelius and WJ Federspiel Dept. Biophysics, Univ. of Roch., Rochester, NY 14642 Dept. Biomed. Eng., Boston Univ., Boston, MA 02215

The relationships currently used to predict red cell $(R B C)$ distribution downstream of arterial \& venous type bifurcations in simulations of microvascular network blood flows are inadequate for prediction of local parameters. A major factor in such relationships is the radial distribution of cells across the vessel lumen. In in-vivo networks, the distances between successive bifurcations are usually 100-300 microns; the effective $\mathrm{RBC}$ radial diffusivity (D) is so small that the luminal distribution of RBC established in a downstream vessel of a bifurcation is presented to the inlet of the next bifurcation. Thus, the relationship for the RBC distribution at a bifurcation is strongly influenced by the prior flow history of the blood. The value of $D$ has been determined in in-vitro venous-type bifurcations to be about 10 . $\mathrm{cm}^{2} / \mathrm{s}$. The vessel length required to establish the equilibrium luminal distribution of RBC from the convergent flows exceeds $0.75 \mathrm{~cm}$ in a bifurcation of 75 micron vessels. Both in-vitro and in-vivo data will be presented, to show the impact of flows in successive bifurcations on RBC distributions at individual bifurcations.

\section{7}

BLOOD FLOW RESISTANCE OF MICROVESSELS IN VIVO: IMPLICATTONS OF COMPARISONS BETWEEN PREDICTED AND OBSERVED NETWORK FLOWS

T.W. Secomb, ${ }^{1}$ A.R. Pries, ${ }^{2}$ T. Gessner ${ }^{2}$ and P. Gaehtgens ${ }^{2}$

iDept. of Physiology, Univ. of Arizona, Tucson AZ 85724;

${ }^{2}$ Dept. of Physiology, Freie Universităt Berlin, D-1000 Berlin, Germany.

The resistance to blood flow in the peripheral circulation depends on the architecture of microvascular networks and on the rheological behavior of blood. Studies of blood flow in narrow glass tubes have demonstrated that apparent viscosity decreases strongly with decreasing tube diameter, reaching a minimum in tubes of about $7 \mu \mathrm{m}$ diameter (Fahraeus-Lindqvist effect). In comparison, direct measurements in individual microvessels in vivo have yielded higher values of apparent viscosity, but have been limited by experimental difficulties. The present study is based on observations of six microvascular networks in the rat mesentery, in which flows (for three networks) and hematocrits were measured in all segments (between 328 and 913 per network). Flows and hematocrits were also predicted using theoretical simulations based on the architecture of the networks studied, assuming that apparent viscosity varies with diameter and hematocrit as observed in vitro (in-vitro viscosity law). Segment-by-segment comparisons showed substantial deviation between observed and predicted values. A significant part of this deviation could not be accounted for based on known sources of measurement and simulation errors. Therefore, further simulations were carried out assuming different viscosity laws. The lowest deviation between observed and predicted values was found assuming a law in which apparent viscosity, for diameters below about $50 \mu \mathrm{m}$, is substantially higher than in-vitro values and increases with decreasing vessel diameter. These results indicate that terminal blood vessels exhibit higher resistance to blood flow than would be assumed on the basis of rheological studies in glass tubes. Supported in part by NIH Grant HL34555.
258

WHOLE ORGAN PRESSURE-FLOW CURVES PREDICTED FROM MICROVASCULAR HEMODYNAMICS IN SKELETAL MUSCLE.

G. W. Schmid-Schönbein, D. Sutton, S.-Y. Lee, F. A. DeLano Institute for Biomedical Engineering, University of California, San Diego, La Jolla, California 92093-0412

The quantitative prediction of whole organ hemodynamics from the properties of individual microvessels, the microvascular anatomy, and from the flow of blood cells in the microcirculation represents an important objective in understanding the cardiovascular system. The microvasculature in skeletal muscle can be classified into arcade arterioles with several inflows from central arteries, transverse (terminal) arterioles, capillaries, collecting venules and arcade venules with multiple outflows into central veins. All classes of microvessels are distensible so that steady flow in resting skeletal muscle depends both on the arterial and venular pressure, vessel distensibility, plasma viscosity, and the initial radius and length of the vessels. After integration over all vessel hierarchies, the theoretical relationship between arterial flow and pressure can predict the measured flow of plasma with differen Newtonian viscosities and vessels distensibilities within experimental error. In contrast, the prediction with non-Newtonian viscous properties of the blood at different hematocrits or leukocyte counts, which is based on symmetric models of cell position in individual blood vessels, tends to underestimate the measured flows into the muscle. The measured flows are overestimated at all arterial pressures. The origin of this discrepancy between theory and experiment is currently unresolved and may be related to nonsymmetric cell positions in microvessels. Supported by USPHS grant HL 10881 .

\section{9}

INFLUENCE OF CAPILLARY UNIT STRUCTURE ON PREDICTION OF SKELETAL MUSCLE BLOOD FLOW.

T.C. Skalak and H.H. Hsiung

Dept. of Biomedical Engineering, University of Virginia, Charlottesville, vA 22908

Using accurate anatomical vascular models, a quantitative prediction of whole organ blood flow may be made if realistic descriptions of vessel mechanics and blood viscosity are known. While the active mechanical behaviour of vessels and the detailed blood cell distributions are the subject of intense current investigation, the anatomical arrangement of vessels in solid organs is an important determinant of overall flow resistance and is currently unavailable for any organ. In particular, the arrangement of vessels at one level of microcirculation may yield accurate flow predictions at that level, while whole organ flow predictions remain inaccurate due to imprecist description of microvascular unit interconnections. Three-dimensional reconstructions of arterio-venous capillary units in rat gracilis muscle were performed using serial 1 um sections. Five units were reconstructed for both WKY (normotensive) and SHR (spontaneously hypertensive) rats. Blood flow in the units was computed using measured vessel dimensions, an in vivo viscosity dependent on shear rate, and experimental perfusion pressures. Total unit flow averaged $0.33\left(10^{3} \mathrm{~mm}^{3} / \mathrm{sec}\right)$ for WKY and 0.58 for SHR. Experimental flow estimates from earlier work in rat skeletal muscle were 1.19 for WKY and Experimental flow estimates from earlier work in rat skeletal muscle were 1.19 for WKY and
1.17 for SHR. Comparison of the predicted flows with the experimental flows suggests that each tranverse arteriole supplies about 3.5 units in WKY and about 2 units in SHR. The notion that a transverse arteriole supplies two units (a unit pair) is consistent with many previous findings based on anatomical reconstruction of thin muscles or superficial units in thick muscle. However, the comparison also suggests that different anteriole/capillary unit arrangements exist in different strains, and must be known in order to quantitatively predict whole muscle flows using capillary unit structure as a fundamental building block. In turn, computed flow heterogeneity showed that muscle fiber type and arteriolar structure are significant determinants of flow distribution within units. Supported by NIH HL-39680.

\section{0}

FUNCTIONAL SIGNIFICANCE OF INTERCONNECTIVITY IN CAPILLARY NETWORXS A. G. Hudet z Dept. of Physiology, Medical college of Wiscongin, Milwaukee, wI

In most tissues arterio-venoug capillaries are interconnected by several anastomosing segments. The functional gignificance of this interconnectivity is, however, unclear. In planar polygonal (mesentery) and three-dimensional (brain) networks, arterio-venoug and anastomosing capillary segments are not easily discernible based on their branching orders. Ingtead, erythrocyte flow pathways may reflect the functional organization of the capillary network. Flow pathways may also provide a clue to understand how microvascular networks develop from primitive sinusoid plexuses. Computer simulations suggest that local adaptation of vessel diameter to flow (shear stress) alone is ingufficient to predict the developmental differentiation of interconnected microvascular network. clearly, capillary transport related andor angiogenic factors must be involved. Additionally, capillary interconnections may participate in intramural vessel-to-vessel communication which may coordinate adjustments of vascular caliber to flow. Another related question is whether interconnections play a role in maintaining network perfusion when capillary density is altered. Computer models predict a nonlinear relationship between network hydraulic resistance and the fraction of nonperfused capillaries. This suggesta that, due to interconnectivity, the surface area for transcapillary exchange may be regulated by capillary recruitment without an effect on arteriolar pressure or flow. Supported by the NSF grant BCS-9001425. 
261

A NOVEL BIOMECHANICAL MODEL FOR THE MYOGENIC RESPONSE IN SKELETAL MUSCLE ARTERIOLES

Shouyan Lee and Geert W. Schmid-Schönbein

Institute for Biomedical Engineering, University of California, San Diego, La Jolla, California, 92093-0412

The microcirculation in the skeletal muscle is controlled by several local regulatory mechanisms in which the myogenic response plays a central role. A biomechanical model for the myogenic response was developed based on two basic assumptions: 1) the arterioles maintain viscoelastic properties during active myogenic vasoconstriction; 2) the active myogenic vasoconstriction or dilatation is the result of a change in reference diameter of the vessel. It was assumed that the reference diameter is a function of the local transmural pressure.

A sequence of in vivo experiments was performed to test the model for various pressure histories on arterioles in the rat cremaster muscle using a modified "box method". The model was also tested against a range of data in the literature.

The results show that the theoretical predictions are in good quantitative agreement with both in vivo and in vitro experimental observations. Application of the model to the prediction of pressure-flow response in a single vessel shows that the myogenic model qualitatively produces typical experimental phenomena of autoregulation and reactive hyperemia. (This research was supported by NSF Grant DCB-88-19346)

\section{2}

LEUKOCYTE-CAPLLLARY PLUGGING AND NETWORK RESISTANCE IS INCREASED IN SKELETAL MUSCLE OF DIABETIC RATS

Anthony G. Harris', Thomas C. Skalak', and Diane L. Hatchell ${ }^{2}$

${ }^{1}$ Dept of Biomedical Engineering, Univ. of Virginia, Charlottesville, VA 22908

${ }^{2}$ Dept of Ophthalmology, Duke University Medical Center, Durham, NC, 27710

Although diabetic retinopathy is a leading cause of blindness, the mechanisms underlying the disorder remain unresolved. Recent studies have reported both an increase in viscosity and a decrease in filterability of the blood in diabetes, as well as increased activation of monocytes and granulocytes. These rheological effects have been implicated in capillary closure which is an early pathological change in diabetic retinopathy. The objective of this study was to quantitatively measure in vivo the resistance increase in capillary networks due to leukocyte-capillary plugging during diabetes. Intravital measurements of plugging durations and frequencies were made throughout capillary networks in the spinotrapezius muscle of anesthetized rats subjected to streptozocin (STZ) induced hyperglycemia. These data were used to estimate the increase in microvascular flow resistance due to leukocyte plugging. The increase averaged $13.0 \%$, which is significantly different $(P<0.05)$ than the $1.1 \%$ observed in previous experiments with notmal rats (Harris and Skalak, Am. J. Physiol., 264: H909-H916). Although the total white cell count was normal, the diabetic animals exhibited a significantly increased percentage of monocytes. A small but significant decrease in capillary diameter in the diabetic animals was also observed. Thus, leukocytes have a significant impact on microvascular hemodynamics in diabetic animals and leukocyte-capillary plugging may be an important mechanism of capillary closure and subsequent microvascular dysfunction in diabetic retinopathy.

Supported by NIH HL-39680 and EY-02903.

\section{3}

PREDICTION AND VALIDATION OF PARTICLE DISTRIBUTIONS IN SYNTHETIC AND NATURAL BLOOD ADMTXTURES IN BRANCHED NETWORKS Peter A. Russell1, B.S., Anthony J. McGoron ${ }^{2}$, Ph.D.,

S. Abdallah ${ }^{1}$, Ph.D., Ronald W. Millard ${ }^{2}$, Ph.D.

${ }^{1}$ Department of Aerospace Engineering

${ }^{2}$ Department of Pharmacology and Cell Biophysics

University of Cincinnati, Cincinnati, Ohic 45221

Fluorocarbon (FC) based blood substitutes (particle size $0.2 \mu \mathrm{m}, \rho=1.9 \mathrm{~g} / \mathrm{mi}$ ) mixed with whole blood (red blood cel] size $-5 \mu \mathrm{m}, \rho=1.06 \mathrm{~g} / \mathrm{ml}$ ) are postulated to display unique radial distributions in steady state laminar flow conditions within blood vessels in vivo. Preferential thargination predicted for FC particles would contribute to different admixtures with blood cells in "dant conduits. We aply computational techniques to solutions of three-dimensional Navier-Stokes equations using unique Cartesian finite-difference grids to analyze and predict flow outcomes in $90^{\circ}$ branched tubes. Numerical solutions of particle trajectories are then produced based on viscous forces associated with the determined flow field. Experimental companion studies are performed in vitro for $200 \mu \mathrm{m}$ to $3000 \mu \mathrm{m}$ diameter models and are used to validate the simulations.
264

PATHWAYS OF FLOW-INDUCED DILATION IN PIGLET ISOLATED CEREBRAL ARTERIES.

L. A. Shimoda, N. A. Norins and J. A. Madden.

Marquette University, The Modical College of WI and VAMC, Milwaukee, WI.

Cerebrovascular hemorthage ( $\mathrm{CH}$ ) is perhaps the most widely occuring neurologic disorder seen in neonatal intensive care units. It is widely accepted that $\mathrm{CH}$ is primarily caused by
fuctuations in cerebral blood flow, and increases in flow have been shown to cause dilation in fuctuations in cerebral blood flow, and increases in flow have been shown to cause dilation in
cerebral arteries. Since the mechanism by which CH occurs is unknown the purpose of this cerebral arteries. Since the mechanism by which CH occurs is unkmown the purpose
study was to examine possible pathways by which flow-induced dilation modulates cerebrovascular tone. Middle cerebral arteries from 2-14 day old piglets were mounted on glass cannulas and bathed in and perfused with physiologic saline solution (PSS) at $37^{\circ} \mathrm{C}$. An electronic system controlled pressure by changing oufflow resistance and a syringe pump provided constant flows. In several studies a micropipette was inserted into the lumen of the artery to verify luminal pressure. Vessel diameter was measured with a video system. The vessels equilibrated at $20 \mathrm{~mm}$ Hg for $60-90$ minutes. Flow rates were increased from 0 to 2.24 vessels equilibrated at $20 \mathrm{~mm} \mathrm{Hg}$ for $60-90$ minutes. Flow rates were increased from 0 to 2.24
$\mathrm{ml} / \mathrm{min}$ at $20 \mathrm{~mm} \mathrm{Hg}$ and vessel diameter measured (F/D curves). The F/D curves were repeated $\mathrm{ml} / \mathrm{min}$ at $20 \mathrm{~mm} \mathrm{Hg}$ and vessel diameter measured (F/D curves). The F/D curves were repeated
in the presence of nitro-L-arginine (NLA), indomethacin (IND), 6-hydroxydopamine (6-OHDA) in the presence of nitro-L-arginine (NLA), indomethacin (IND), 6-hydroxydopamine (6-OHDA)
and in PSS with $\mathrm{Na}^{+}$reduced or the $\mathrm{Ca}^{2+}$ removed. F/D curves were also done on arteries that did not respond to potassium chloride after being bathed in deionized water (non-physiologically active). Increasing flow at constant pressure resulted in a biphasic response; constriction at low flows and dilation at higher ones. Addition of NLA, IND and 6-OHDA did not eliminate the flow-induced dilation. Reduction of $\mathrm{Na}^{+}$and removal of $\mathrm{Ca}^{2+}$ also failed to eliminate dilation. In addition, non-physiologic anteries, in which the endothelial and/or smooth muscle cells were destroyed, exhibited a reduced dilation. These data indicate that nitric oxide, arachidonic acid metabolites, adrenergic nerve endings, $\mathrm{Na}^{+}$and $\mathrm{Ca}^{2+}$ do not appear to play a role in flowinduced dilation. That dilation at high flows was never eliminated leads to the hypothesis that induced dilation. That dilation at high flows was never eliminated leads to the hypothesis that
flow-induced dilation may be due to structural components of the vascular wall, such as flow-induced dilation may be due to structural components of the vascular wall, such as
deformation of the collagen/elastin matrix due to increases in shear stress with increased flow.

265

CHAOTIC TIME SERIES ANALYSIS OF HEMATOCRIT VARIATIONS PREDICTED BY A MATHEMATICAL MODEL OF MICROVASCULAR NETWORK FLOW A. M. Barsoumt, D. R. Sainanitt, C. G. Ellist, M. F. Kianie, G. R. Cokelet ${ }^{\circ}$ 'Dep'ts of Medical Biophysics, *Electrical Engineering, The University of Western Ontario, London, Canada, Dep't of Biophysics

The University of Rochester, Rochester, N.Y.

Hematocrit distributions in two vessels of a complex mesenteric capillary network were generated using a mathematical model. The model accounts for the non-linear flow properties of blood, the non-uniform distribution of cells in each vessel, and cell partitioning at the bifurcations. The movement of red blood cells in the network are tracked; hence the model is able to simulate the oscillations in local hemodynamic variables due to hemorheological mechanisms. Chaotic time series analyses consisting of the computation of the autocorrelation function and the correlation dimension were applied on each vessel to analyse the temporal hematocrit fluctuations over a seven minute time period. The results suggest low dimensional dynamics with a correlation dimension between 2 and 3 . Time series analysis was also done on "surrogate datasets." These datasets were constructed from the original hematocrit distribution data. They are essentially comprised of randomly distributed data, but share certain characteristics with the original data, such as the power spectrum. Comparison of the results of analysis done on the surrogate datasets with those of the original data results of analysis done on the surrogate datasets with those of the original data can be quantitatively differentiated from linearly correlated noise.

This study was supported by HL18208 and IF32HL08506, and the Heart \& Stroke Foundation of Ontario. Dr. Ellis is a Career Investigator of the H.S.F.O.

\section{6}

MATHEMATICAL MODELING OF THE EFFECTS OF PERIODICAL BOUNDARY CONDITIONS ON TEMPORAL VARIATIONS IN LOCAL FLOW PARAMETERS IN MICROVASCULAR NETWORK BLOOD FLOW M.F. Kiani, G.R. Cokelet and A.R. Pries

Department of Biophysics, University of Rochester, Rochester, NY 14642, and Department of Physiology, Freie Universitat Berlin, Berlin 33, Germany

A mathernatical model of microvascular network blood flow, which accounts for the non-linear flow properties of blood and the non-uniform distribution of cells in each vessel as well as cell partitioning at bifurcations is developed that is able to simulate the temporal variations in local flow parameters (e.g. $H_{d}$ and blood velocity) by tracking the movement of red blood cells in microvascular networks. The model was used to simulate the effects of periodical boundary conditions on local flow parameters in 4 rat mesenteric networks. Sinusoidal flow boundary conditions in the following form were imposed on all inflow boundary vessels: $Q=\left[1+A^{*} \operatorname{SIN}\left(F^{*} t\right)\right]^{*} Q_{m}$, where $Q$ is volumetric blood flow rate into the boundary vessel, $A$ is amplitude (from 0.2 to 0.5 ), $F$ is frequency (from 5 to 300 Cycles Per Minute, CPM), $t$ is time, and $Q_{m}$ is the average in vivo measured $Q$ into the boundary vessel. The results indicate that temporal variations in flow parameters are only affected by sinusoidal boundary conditions when F is lower than $10 \mathrm{CPM}$. Changing A did not affect the temporal variations in flow parameters. Average total resistance in the network was not affected by imposition of periodical boundary conditions. The mathematical model presented here only accounts for the hemodynamic factors; the effects of having periodical boundary conditions is expected to be more pronounced if myogenic factors and vascular compliance
are taken into account. (Supported by HL18208, 3F32HL08506, and PSC) 
267

A MODEL OF DRUG DISTRIBUTION FOR TWO-STEP APPROACHES TO CANCER THERAPY

\section{L.T. Baxter, F, Yuan, and R.K. Jain}

Massachusetts General Hospital and Harvard Medical School

Department of Radiation Oncology, Cox-7, Boston, MA 02114

The rationale behind two-step approaches to cancer therapy is to combine the selectivity of certain large molecular weight agents (e.g., monoclonal antibodies), with the high permeability and mobility of small molecular weight drugs (e.g., haptens). This approach seeks to avoid the uneven uptake and distribution of antibodies in solid tumors while providing high tumor to normal tissue uptake ratios.

Previously we have developed a lumped parameter model to predict average tumor concentrations of bifunctional antibodies and haptens (Yuan, Baxter, \& Jain. Cancer Res. 51 3119-3130, 1991) and physiological models for antibody distribution (Microvascular Res. 37 77-104, 1989 and 41: 252-272, 1991). A corresponding distributed parameter model has now been developed to predict the spatial distribution of antibodies and low molecular weigh agents on both microscopic (surrounding individual blood vessels) and macroscopic (whole tumor) length scales. The effects of binding affinity, blood vessel density, antigen concentration, and the dose and schedule of injections have been studied.

Model results suggest: clearing circulating antibody from the bloodstream before the injection of hapten increases the uprake ratio; the use of haptens with bifunctional antibodies reduces but does not eliminate the penetration retardation caused by high affinity perivascular binding.

268

TRANSPORT OF IMMUNOTOXINS INTO SOLID TUMORS

C. Sung, R.J. Youle, and R.L. Dedrick

National Institutes of Health

Immunotoxins are conjugates of antibodies and protein toxins which can be designed to bind to antigens associated with tumor cells and thereby form tumor-selective toxins. The pharmacokinetics of protein toxins and immunotoxins that varied in molecula weight and binding characteristics were studied in solid thabdomyosarcoma tumors grown subcutaneously in athymic mice. Plasma-to-tumor transport rates and apparent binding parameters were obtained by means of a compartmental model, but a major limitation of this modeling approach is the assumption that the immunotoxin is homogeneously distributed throughout the fumor. Spatially distributed models require more information regarding a tumor's microvascular and interstitial properties than compartmental models do, but offer valuable insights into factors that may lead to a heterogenous distribution. We have performed quantitative autoradiography of tumo sections in order to examine the spatial distribution of immunotoxins. Immunotoxins that bind strongly to this tumor showed a punctate distribution, presumably due to binding of the immunotoxins within narrow cuffs surrounding permeable vessels. Lowering the molecular weight of the binding immunotoxin from $210 \mathrm{kDa}$ to $110 \mathrm{kDa}$ did not lead to significantly greater homogeneity. Immunotoxins and protein toxins with low binding were fairly homogeneously distributed. Current investigations on other approaches attempting to overcome the heterogeneity of immunotoxin distribution in tumors will also be discusssed.

269

THE MICROPHARMACOLOGY OF ANTIBODIES IN BULK TUMORS AND MICROMETASTASES.

J.N. Weinstein, C. Sung, W. van Osdol, M. Juweid, T. Saga, J. Sato, T. Heya, R.D. Neumann, and R.L. Dedrick. National Institutes of Health, Bethesda, MD 20892

For a number of years we have been developing mathematical models for MAD and MAD conjugate penetration into prevascular, densely packed nodules representing either primary or metastatic tumors (van Osdol et al., Ca. Res. 51:4776, 1991; Fujimori, et al., Ca Res. 51: 4821, 1991; Weinstein et al, Ca. Res. 52: 2747s, 1992). Those analyses combined several aspects of antibody pharmacology: global (whole body) pharmacokinetics: transcapillary transport into normal tissue interstitium surrounding the nodule; diffusion into the nodule; non-specific binding or partitioning: specific binding to tumor antigen; metabolism; dehalogenation; lymphatic outflow; and antigen density. Predictions from those models were consistent with the "binding site barrier" hypothesis (Weinstein et al., Annals N.Y. Acad. Sci. 507: 1987, 1987; Fujimori et al., Ca. Res. 49: 5656,1989 ), which posits that ligands such as MAbs can be prevented from penetrating tissues or tumors by the very fant of succe as MAbs can be prevented from penetrating tissuen antigen density and/or high alfinity can reduce the absolute concer
distances of a hundred or more microns from the nearest capiliary.

A large number of studies from various laboratories have shown heterogeneous MAb distribution in solid tumors, and a number of likely mechanisms for that heterogeneity have been adduced (see Jain and Baxter, Ca. Res. 48: 7022, 1988 Baxter and Jain, Microvas. Res. 41: 5, 1991). However, tormal demonstration that binding is the cause requires simultaneous measurement of the location of MAb, antigen, control immunoglobulin, and blood vessels. We have now done those experiments in guinea pigs with bulk intradermally injected line 10 carcinoma (Juweid, et al. Ca. Res 52.5144 , 1992) and in micrometastases of the same carcinoma (Juweid, et al., Ca. Res. 52: 5144 , 1992) and in micrometastases of the same lumor (Proc. Natl. Acad. Sci., submitted). The site barrier" hypothesis.
270

OXYGEN TRANSPORT TO TUMOR TISSUE BY MICROVASCULAR NETWORKS

T.W. Secomb, ${ }^{1}$ R. Hsu, ${ }^{1}$ M.W. Dewhirst, ${ }^{2}$ E.T. Ong ${ }^{2}$ and J.F. Gross ${ }^{1}$

${ }^{1}$ Dept. of Physiology, Univ. of Arizona, Tucson AZ 85724;

2Div. of Radiation Oncology, Duke University Medical Center, Durham NC 27710

The rate of oxygen consumption and the spatial arrangement of blood vessels strongly influence the distribution of partial pressure of oxygen ( $\left.\mathrm{PO}_{2}\right)$ in tumor issue and the occurrence of hypoxic, radiation-resistant regions. We present experimental and theoretical studies of these two factors and their effects on tumor oxygenation. Observations were made in the tissue between two glass plates in a rat dorsal skin flap preparation, in which mammary adenocarcinomas ( $R 3230 \mathrm{AC}$ ) were introduced and allowed to grow. At 9 days, one glass plate was removed and a Whalen $\mathrm{PO}_{2}$ microelectrode was used to measure PO profiles ( $100 \mu \mathrm{m}$ to $300 \mu \mathrm{m}$ in length) in tissue between two vessels. The local consumption rate was estimated for best fit between measured profiles and profiles predicted theoretically, assuming two-dimensional diffusion. Results ranged from 0.83 to $1.84 \mathrm{~cm}^{3} \mathrm{O}_{2} / 100 \mathrm{~g} / \mathrm{min}$, consistent with determinations for macroscopic regions. In further preparations, networks of vessels were mapped, giving data on length, diameter and flow velocity in each segment. Corresponding three-dimensional simulations were carried out using a Green's function method. Predicted cumulative frequency distributions of $\mathrm{PO}_{2}$ were compared with predictions of a Krogh-type model with the same density of vessels, but with uniform spacing. The latter approach underestimated the extent of the hypoxic tissue. Predicted maximum consumption rates that could be sustained without tissue hypoxia were substantially lower than those obtained from the Krogh-type model. Thus, the heterogeneous structure of tumor microcirculation can substantially infiuence the occurrence of hypoxic micro-regions. Supported by NIH Grants CA40355 and HL07249.

\section{1}

A STATISTICAL VIEW OF HEAT TRANSFER IN PERFUSED TISSUE

James W. Baish

Department of Mechanical Engineering, Bucknell University, Lewisburg, PA 17837

Heat transfer in perfused tissue has historically been modeled by modified versions of the heat conduction equation known as bioheat equations. Such continuum models have been useful for estinating average tissue temperatures, but have been the subject of considerable controversy in recent years. After reviewing the fundamental difficulties with the existing approaches, a new perspective will be presented that clarifies many of the underlying issues and leads to a new model for tissue transport. The new method predicts a probability density function for the tissue temperature, not just a local average. This result carries information on the most probable temperature at a point and the uncertainty in that temperature due to the proximity of thermally significant blood vessels. A sample implementation illustrates the dependence of the probability density function on the flow rate of the blood and the laws governing the growth of blood vessels. The model is useful for a variety of clinical applications involving tissue heating and fundamental studies of tissue heat transport, and should extend to problems of tissue oxygen diffusion and other forms of mass transport. 
272

MATHEMATICAL SIMULATION OF GAS TRANSPORT AND ACID-BASE REGULATION BY BLOOD FLOWING IN MICROVESSELS

Nancy S. Huang, J. D. Hellums and John S. Olson*

Department of Chemical Engineering and Department of Biochemistry and Cell Biology* Rice University, Houston, TX, 77251-1892

An investigation is made of the coupling between the $\mathrm{O}_{2}$ and $\mathrm{CO}_{2}$ transport by blood flowing in microvessels. The blood is treated as two continuous coexisting phases: a RBC phase and a plasma phase. The microvessel is divided into two regions: the central, RBC-rich and the outer, cell-free region. The radial distribution of RBCs and transport of various species due to bulk convection are taken into account. Chemical and transport processes which are included in the model are (1) interactions of hemoglobin with $\mathrm{O}_{2}$ and $\mathrm{CO}_{2}$ (2) the Bohr and Haldane effects, (3) $\mathrm{CO}_{2}$ hydration-dehydration reactions, (4) buffering actions of hemoglobin and plasma proteins, and (5) anion exchange actoss the red-cell membrane. The governing equations of the model subjected to the imposed inlet and boundary conditions are solved numerically to provide the concentration distributions of various species in blood that are important in the simultaneous gas exchange and $\mathrm{pH}$ regulation process. Predictions of the new model of simultaneous $\mathrm{O}_{2} / \mathrm{CO}_{2}$ transport by flowing blood are shown to be in excellent agreement with prior workers' experimental results from artificial membrane tubes. A previous mathematical model which treats blood as a homogeneous continuum and uses a local chemical equilibrium approximation to describe the gas transport is shown to satisfactorily predict the amount of $\mathrm{O}_{2}$ transport for blood oxygenation accompanied by $\mathrm{CO}_{2}$ elimination. However, the previous model significantly underpredicts $\mathrm{O}_{2}$ transfer for blood deoxygenation accompanied by $\mathrm{CO}_{2}$ uptake. Furthermore, the previous model disagrees substantially with the $\mathrm{CO}_{2}$ transport results under both oxygenation and deoxygenation conditions.

273

DOES OXYGEN DIFFUSE OR DISPERSE IN MYOCARDIAL TISSUE? R. W. Schubert and J. E. Fletcher

Louisiana Terh University, BME Dept., Ruston, LA 71272 "NIH Laboratory of Applied Studies, Bethesda, MD 20892

Oxygen transport simulations based on Krogh geometry with tissue radial and axial diffusion demonstrate that histograms are sensitive to the axial diffusion coefficient and capillary length, but insensitive to the radial diffusion coefficient. Histograms similar to experimental histograms were obtained when either the axial diffusion was enthanced by a factor of $\theta$ GR capiliary length was reduced to $40 \%$ of anatomical length. It has been suggested to us that staggered capillary entrances and exits would produce a functionally shorter capillary, but it is not clear if this effect would reduce the length from 500 to 200 micrometers. Alternately, the working myocardium is anything but passive. Actin-myosin crass-bridges make and brake. Trans-mitochondrial water and hydrated calcium flux. Sarcomere shorten. These mechanisms could give the appearance of an enhanced diffusivity. we tentatively conclude that the assumed passive diffusive oxygen transport in heart tissue is augmented by the activity of contracting heart. This phenomena seems best described as oxygen dispersion.

INTRACELLULAR DIFFUSION OF OXYGEN: MEASUREMENTS AND MATHEMATICAL MODELS

Roland N. Pittman, Timothy B. Bentley, Abhijit Dutta* and Aleksander S. Popel* Department of Physiology, Medical College of Virginia, Richmond, VA and *Department of Biomedical Engineering, Johns Hopkins University, Baltimore, MD

In vivo data on $\mathrm{O}_{2}$ transport in arterioles of the hamster retractor muscle indicate that observed diffusive $\mathrm{O}_{2}$ losses are an order of magnitude higher than predicted by mathematical models in which the tissue is treated as a continuum. A possible resolution of this discrepancy is that the in vivo permeability of the tissue to $\mathrm{O}_{2}$ (i.e., $\mathrm{KO}_{2}=$ diffusion coefficient $\mathrm{x}$ solubility) is much higher than the in vitro value used in the model. We have measured $\mathrm{O}_{2}$ diffusion in the retractor muscle from 11 to 40 ${ }^{\circ} \mathrm{C}$ and found that $\mathrm{KO}_{2}$ rises rapidly in a temperature range associated with phase transitions in lipid membranes. $\mathrm{O}_{2}$ diffusion within cells may be heterogeneous with more rapid diffusion along intracellular pathways of high $\mathrm{O}_{2}$ solubility, such as mitochondria. We have analyzed our data as a function of temperature using theoretical models of diffusion in two-phase media. By assuming muscles to be composed entirely of aqueous cytosol and lipids, we determined cytosolic $\mathrm{KO}_{2}$ as a function of temperature by matching the experimental values with those of model predictions using in vitro values of lipid $\mathrm{KO}_{2}$. Cytosolic $\mathrm{KO}_{2}$ ranged from $50 \%$ of water $\mathrm{KO}_{2}$ (low temperature) to $90 \%$ of water $\mathrm{KO}_{2}$ (high temperature). An upper bound for cytosolic $\mathrm{KO}_{2}$ was calculated using a physiologic value for protein volume fraction, and lipid $\mathrm{KO}_{2}$ was obtained using this cytosolic $\mathrm{KO}_{2 .}$ A model with a parallel arrangement of lipid and cytosol yielded a value of lipid $\mathrm{KO}_{2}$ that was $71 \%$ higher than the in vitro value. Comparison of three different muscles exhibited the same trend of higher $\mathrm{KO}_{2}$ with higher lipid content. These results warrant additional measurements of $\mathrm{KO}_{2}$, especially for oxidative muscles with high lipid content.
275

DIFFUSIONAL SMOOTHING OF LOCAL $\mathrm{pO}_{2}$ GRADIENTS IN THE PRESENCE OF FRACTAL FLOW DISTRIBUTIONS IN THE HEART J. I. S. Chan, R. B. King and J. B. Bassingthwaighte,

Center for Bioengineering, Univ. of Washington, WD-12, Seattle, WA 98195

Steady state gradients in tissue concentrations reach a balance where influences of delivery and removal (by convection and consumption) exactly balance those of diffusion and permeation within each unit. For substances of high lipid solubility and diffusivity, net diffusion occurs between neighboring units when they are parametrically dissimilar. We modeled oxygen profiles for intramyocardial flow distributions using the known fractal characteristics of the neighbor-to-neighbor variation in blood flows. Fractal flow profiles in 2-space or 3-space are fractional Brownian densities, not fractal surfaces (which are much smoother). Diffusion between neighboring regions smooths the concentration profiles. In organs with low fractal dimension and high Hurst coefficient (high neighbor to neighbor correlation in flows), diffusional interactions have less influence than when flows are random, because the gradients between neighboring units are smaller when correlation is fractal. For oxygen this is imporant in reducing the effect of local derecruitment or variations in intercapillary distances. Diffusional smoothing is less effective when local oxygen consumption is high. Mappings of intratissue concentrations aid in understanding the events. (Supported by HL50238 and RR1243.)

276

EVIDENCE OF A DIFFUSIVE OXYGEN SHUNT IN STRIATED MUSCLE 'J. Christopher Stein, ${ }^{2}$ Christopher G. Ellis and ${ }^{1}$ Mary L. Ellsworth 'St. Lovis University School of Medicine, St. Louis, MO, USA and ${ }^{2}$ University of Western Ontairo, London, Ontario, Canada

Diffusive oxygen loss across the walls of the arterioles has been demonstrated in striated muscle by numerous investigators. However, whene this lost oxygen diffuses to has been the subject of much speculation. One possible sink could be venules running in parallel with the arteriolar network. Even though this diffusive shunt has been considered of minor importance, no other destination has been found which alone could account for the observed magnitude of the oxygen loss. The objective of this study was to determine if there is a significant increase in the level of oxygen in the blood as it traverses the venous portion of the microvasculature. Oxygen tensions $(\mathrm{PO}$ ) were determined simultaneously at the venous end of capillary networks and in the firs order venule in the hamster, retractor muscle during nonhypoxic $\left(\mathrm{F}_{\mathrm{i}} \mathrm{O}_{2}=0.3\right.$ and 0.21$)$ and hypoxic $\left(\mathrm{F}_{\mathrm{j}} \mathrm{O}_{2}=0.1\right)$ ventilation in conjunction with systemic arterial and venous blood gases. Capillary data on oxygen saturation ( $\mathrm{SO}_{2}$ ) and red cell supply rate (SR) were measured using in vivo video microscopy and image analysis techniques. Capillary $\mathrm{PO}_{2}$ was estimated from the measured $\mathrm{SO}_{2}$ using the appropriate standard $\mathrm{P}_{\mathrm{so}}$ and corrected for the $\mathrm{PCO}_{2}$ and $\mathrm{pH}$ expected within the capillary network. Venular $\mathrm{PO}_{2}$ was measured using oxygen microelectrodes. We found significant increases in $\mathrm{PO}_{2}$ of the blood as it traversed the venular network of $9.9 \mathrm{mmHg}$ while the animal inspired $30 \% \mathrm{O}_{2}$ and $4.0 \mathrm{mmHg}$ when the animal breathed room air. There was no significant difference between capillary and venular data during hypoxic ventilation. Weightiag the capillary $\mathrm{SO}_{2}$ data by the SR had no effect on the result suggesting that the shunt is not a fuactional one. Since the magnitude of the $\mathrm{PO}_{2}$ increase was dependent on the level of inspired oxygen, these results are consistent with the presence of a diffusional shunt for oxygen between arterioles and venules, which disappears during hypoxia. (Supported by NIH: HL-39226 (MLE and JCS) and RCDA HL-02602 (MLE) and Heart and Stroke Foundation of Ontario, (CGE))

277

EFFECTIVE DIFFUSIVITIES OF OXYGEN IN IMMOBILZED CELL SYSTEMS: A MONTE CARLOSTUDY

H.M. Buettner ${ }^{1}$ [Speaker], M.R. Riley 1, F.J. Muzzio1, and S.C. Reyes ${ }^{2}$

1Department of Chemical and Biochemical Engineering,

Rutgers - The State University of New Jersey Piscataway, N] 08855-0909 2 Corporate Research Laboratories, Exxon Research and Engineering Company,

Immobilized populations of viable cells comprise the essential feature of a wide variety of natural and artificial tissue systems, such as tumors and cell-based arificial organs. The dynamics of these systems are controlled by the interplay of nutrient and product diffusion, cellular metabolism, and cell proliferation. When diffusional resistances become large, dramatic outcomes, such as widespread cell death, are common. An accurate description of the oxygen diffusivity as a function of cell density could greatly enhance the understanding of these systems. To this end Monte Carlo simulations have been implemented to compute effective diffusivities of oxygen in simulated structures of cells distributed in a matrix. The effective diffusivity has been determined to be highly dependent not only on the cell density, but also on the spatial arrangement of the cells. This information can be used to obtain a mean field model of the transport and reaction processes capable of predicting system behavior for a wide range of parameters. 
278

AXIAL BLOOD SATURATION VARIATIONS COMPUTED FROM EXPERIMENTAL $O_{2}$ FLUXES MEASURED NEAR CAT RETTINAL VESSELS

D.G. Buerk and C.E. Riva, Dept. Ophthalmology, Scheie Eye Inst., Univ, of Pa. School of Med., Philadelphia, PA 19104

Quantitative measurements of $\mathrm{O}_{2}$ fluxes $\left(\mathrm{JO}_{2}\right)$ near retinal arterioles and venules in the cat eye have been recently reported (Buerk et al.. Microvas. Res., 45:134-148, 1993). $\mathrm{O}_{2}$ fluxes were calculated from spatially detailed $\mathrm{PO}_{2}$ gradients measured with recessed cathode microelectrodes in the vitreous humor above retinal vessels. Mean retinal arteriole $\mathrm{JO}_{2}$ was $-2.58 \cdot 10^{-6} \mathrm{~mL} \mathrm{O}_{2} / \mathrm{sec} / \mathrm{cm}^{2}$. $\mathrm{JO}_{2}$ near venules was smaller, with both positive and negative values observed. Experimental results suggested that there could be significant $\mathrm{O}_{2}$ losses from arterioles. To investigate this possibility, mathematical models were developed to predict axial blood $\mathrm{O}_{2}$ losses from arterioles. Counter-current exchange ( $A-V$ shunting) between paired vessels, and $\mathrm{O}_{2}$ gains for venules were also modeled. Asymmetrical radial $\mathrm{PO}_{2}$ gradients were expected, due to $\mathrm{O}_{2}$ metabolism of underlying retinal tissue, vascular wall $\mathrm{O}_{2}$ metabolism and $\mathrm{O}_{2}$ in the nonconsuming vitreous humor. Bohr and Haldane effects were also modeled using an oxyhemoglobin saturation algorithm for cat blood (Buerk et a1., J. App 1. Physiol., 67:60-68, 1989), to include $\mathrm{PCO}_{2}$ and $\mathrm{pH}$ changes. Predicted axial variations in blood $\mathrm{O}_{2}$ saturation were relatively small $(<38)$ for normal blood flows. Under some conditions axial variations could affect regulation of blood flow and $\mathrm{O}_{2}$ delivery in the eye. $\mathrm{O}_{2}$ fluxes between parallel vessel pairs may also be a significant factor. Supported by NIH grant EY0 9269.

TISSUE OXYGEN TRANSPORT ENHANCEMENT BY FLUOROCARBON BLOOD SUBSTITUTES: EXPERIMENTAL RESULTS AND A THEORETICAL MODEL

TK Goldstick, RA Linsenmeier, LF Mockros, and CM Waters, Chemical and Biomedical Engineering, Northwestern University, Evanston, IL 60208-3120

Experimental studies with Fluosol DA® (Green Cross Corp., Osaka, Japan) have demonstrated increased oxygen transport to the cat retina following exchange transfusion when breathing $100 \%$ oxygen. This enhancement occurred at greatly reduced hematocrits when the arterial blood carried considerably less oxygen. Studies with a more concentrated emulsion, Oxygent ${ }^{\text {MM }} H T$ (Alliance Pharmaceutical Corp., San Diego, CA), using extremely small amounts of added emulsion, also showed an increase. In these studies there was only a negligible increase in arterial blood oxygen content and no apparent change in either blood $\mathrm{PO}_{2}$ or flow. The mechanism proposed to explain this augmented oxygen transport to tissue depends upon an increased overall mass coefficient between blood and tissue. It is proposed that the fluorocarbon particles reduce the major oxygen transport resistance which resides in the blood plasma, by increasing its oxygen solubility. Furthermore, a near wall excess of these fluorocarbon particles would be expected based on the research of Eckstein and coworkers Such an excess would tend to amplify the effect of even a small amount of fluorocarbon. The enhancement mechanism is currently being studied in highly oxygen permeable (microporous polypropylene) $200 \mu \mathrm{m}$ tubes in an extracorporeal membrane oxygenator. Based on this research, fluorocarbon emulsions would be expected to be clinically useful both in treating tissue hypoxia and improving extracorporeal membrane oxygenator efficiency.

\section{0}

BASAL HYDRAULIC CONDUCTIVITY REFLECTS IN SITU FLOW CONDITIONS B. Thipakorn, D. A. Williams, and V. H. Huxley.

Department of Physiology, University of Missouri, Columbia, MO 65212

Basal measures of hydraulic conductivity $\left(L_{p}\right)$ in in situ capillaries of the frog (Rana Pipiens) mesentery are distributed widely, ranging from 0.1 to $20 \times 10^{-7} \mathrm{~cm} \cdot \mathrm{sec}^{-1} \cdot \mathrm{cmH}_{2} \mathrm{O}^{-1}$. Further, basal $\mathrm{L}_{\mathrm{p}}$ is influenced by calcium flux into cells of the capillary barrier and calcium flux is thought to be flow dependent. Thus, we tested the hypothesis that flow conditions influence capillary $L_{p}$. In situ flow and $L_{p}$ were assessed in six capillaries. First, flow was estimated from measures of frog red blood cell (RBC) velocity using the temporal correlation method. Second, each vessel was then cannulated with a micropipette and $L_{p}$ was assessed from measures of volume flux by the modified Landis technique. The pipette contained marker human red blood cells and $10 \mathrm{mg} \cdot \mathrm{ml}^{-1} \mathrm{BSA}$ in frog Ringer's at $15^{\circ} \mathrm{C}$. Consistent with the initial hypothesis, linear regression analysis demonstrated a positive relationship between $R B C$ velocity and $L_{p}\left(r^{2}=0.92, p=0.002\right)$. This result infers that the wide distributions of basal $L_{p}$ and flow in the intact capillary network are related.

(Support by NIH HL34872, HL42528 and HL07094)
281

SIMULATION OF $\mathrm{CO}_{2}$ TRANSPORT IN AN INTRAVENACAVAL BLOOD-GAS EXCHANGE DEVICE

S. C. Niranjan ${ }^{\ddagger} \ddagger$, J. W. Clark Jr $\ddagger$, K. Y. San $\ddagger$ \&. Bidani

University of Texas Medical Branch, Galveston, Texas 77550 ${ }^{\dagger}$ Rice University, Houston, Texas $77251^{\ddagger}$

A mathematical model of a novel intravascular hollow-fibre membrane oxygenator and carbon dioxide removal device (IVOX) has been developed by considering fluid flow inside and outside of rigid tubes while undergoing simultaneous mass transport. Application of constitutive species mass balances on oxygen and carbon dioxide content result in a nonlinear coupled set of convective-diffusion parabolic partial differential equations in the blood, membrane and gas phases. These are solved using an alternating direction implicit finite difference procedure. Blood is considered to be a homogeneous, incompressible fluid in which reactions are instantaneous while the gas phase is assumed to behave ideally. The membrane phase is modeled as a porous matrix coated on one side with a thin layer of siloxane. Diffusion in all the phases is assumed to be Fickian in nature. Simulation results using this approach indicate the presence of a large resistance to gas exchange on the blood side. Increasing gas and blood flow rates in the system result in increased gas exchange. Transport of $\mathrm{CO}_{2}$ increases directly with the $\mathrm{CO}_{2}$ gradient across the membrane. A clinical strategy of allowing permissive hypercapnia, while controlling respiratory acidosis in patients, may be successfully adopted to enhance $\mathrm{CO}_{2}$ excretion from blood using this device.

282

\section{DEXTRAN TRANSPORT ACROSS BRAIN}

\section{MICROVESSEL ENDOTHELIAL CELL MONOLAYERS}

J.E. Wagner*1 L.V McIntire Ph. ${ }^{1}$ and $P$ A. Whitson Ph.D. ${ }^{2}$

Rice University Department of Chemical Engineering ${ }^{1}$

NASA-JSC Biomedical Operations and Research ${ }^{2}$

Endothelial cells in the brain severely restrict the passage of macromolecules between the blood and the cerebral spinal fluid (CSF) forming what is known as the blood brain barrier (BBB). The existence of a tight barrier between the blood and the CSF can hinder therapeutic drug treatment of brain pathologies such as tumors. The recent ability to culture brain microvessel endothelial cells (BMEC) has made it possible to study controi of the BBB in vitro.

We have explored the perneability of macromolecules as a function of molecular size using dexiran molecules of several sizes. In static culture, the permeability coefficient divided by the diffusion coefficient was found to be a function of molecular size for molecules under $7 \mathrm{~nm}$ Stokes radius and seemed to increase with molecular size for molecules over $7 \mathrm{~nm}$. We also studied the transport of different sized dextrans across BMEC monolayers that were sheared. The results obtained are consistent with a model predicting that small molecules traverse the BBB primarily by traveling through cell junctions while large molecules are transported by an intracellular route.

283

A DISTRIBUTED VASCULAR TRANSPORT MODEL WITH RED CELLS R. B. King, I. S. Chan, and J. B. Bassingthwaighte Center for Bioengineering, Univ. of Washington (WD-12), Seattle, WA 98195

We have extended our distributed, multi-region blood tissue exchange model to include red blood cells (RBC) flowing in the capillary with a velocity different from that of the plasma. The ratio of plasma and RBC velocities can assume any desired value. The model is axially distributed permitting axial concentration gradients in plasma, RBC's, and all the tissue regions. In its current form, all membrane transport and tracer consumption are modeled as linear processes.

The model is useful for analyzing outflow and residue data from positron emission tomography and multiple indicator dilution experiments. It permits examination of the "red cell carriage effect" for tracers that enter the RBC, such as oxygen, rubidium, and thallium. It can also be used to examine the behavior of tracers that do not enter RBC's but bind to other substances in the plasma such as fatty acid which binds to plasma protein. By adjusting the $R B C$ virtual volume, the effect of various values of hemoglobin binding of the tracer can be examined.

Supported by grants RR-01243 and HL50238 from the National Institutes of Health. 
284

TWO-STEP TARGETING OF ANTIBODIES TO TUMORS: THEORETICAL AND EXPERIMENTAI STUDIES

C. Sung, W.W. van Osdol, T. Saga, R.D. Neumann, J.N. Weinstein, and R.L. Dedrick National Institutes of Health

Two-step protocols for targeting monocional antibodies (MAb) to tumors involve injection of a modified MAb capable of binding both to tumor-associated antigen and to a second component that carries a radionuclide or drug. The second component is injected subsequently, after the MAb has distributed throughout the target tumors and been largely cleared from the plasma and normal tissues. We have developed a mathematical model for the analysis of these protocols and applied it to the case of a biotinylated MAb and radiolabeled streptavidin diflusing into small, prevascular, densely cellular nodules representing either primary or metastatic tumors. The model includes the whole body pharmacokinetics, transcapillary transport into normal tissue interstitium surrounding the nodule, binding of MAb to antigen, binding of the second component to the MAb, diftusion into the nodule, and lymphatic outflow from the tissue space Many of the model parameters have been estimated from recent experimental work on metastatic nodules in the guinea pig lung. We examine the theoretical distribution of biotinylated MAb and radiolabeled streptavidin within a tumor nodule and compare them with experimental results. The latter indicate that, under the conditions of these experiments, streptavidin in the tumor progresses from a mainly peripheral distribution at $1.5 \mathrm{hr}$ after injection to a largely homogeneous distribution at $24 \mathrm{hr}$. Homogeneity of the radiolabel was attained more rapidly with this protocol than with one-step MAb targeting. and the tumor-to-normal lung ratio of radiolabel concentration was enhanced, as well.

285

\section{A PHYSIOLOgically-baSed PHARMACOKINETIC MODEL FOR MONOCLONAL ANTIBODIES}

\section{L.T. Baxter, H. Zhu, D.G. Mackensen, and R.K. Jain}

Massachusetts General Hospital and Harvard Medical School, Department of Radiation Oncology, Cox-7, Boston, MA 02114

A physiologically-based pharmacokinetic model for specific monoclonal antibody $\operatorname{IgG}_{1}$ (ZCE025) and its fragments $\left(F\left(a^{\prime}\right)_{2}\right.$ and $\left.F a b\right)$, and for non-specific $\operatorname{IgG}_{1}$ (MOPC21) in normal tissues and a human colon carcinoma xenograft (T380) in nude mice is developed. The model simulates the experimental data on the concentration of these four macromolecules in plasma, urine, heart, lung, liver, kidney, spleen, bone, muscle, skin, G.I. tract and tumor This is the first such model for macromolecules with specific binding. It also uses a two-pore formalism for transcapillary solute exchange which avoids the oversimplifications of unidirectional transport or a single effective permeability coefficient. Comparison of model with our biodistribution data shows that: (1) a physiologically-based pharmacokinetic model for specific and non-specific antibodies is able to explain experimental data using as few adjustable parameters as possible, (2) for antibodies and fragments the tumor itself has no significant influence on the pharmacokinetics in normal tissues; (3) the two-pore formalism for transcapillary exchange describes the data better than a single pore model withou introducing extra adjustable parameters. The implications of this model for scaling up antibody pharmacokinetics from mouse to man will be discussed.

\section{6}

TECHNIQUES FOR ASSESSING GASTROINTESTINAL MOTILITY: POTENTIAL IMPLICATIONS FOR GUIDING ENTERAL FEEDING

S. E. Carlson

Depts. of Peds and Ob/Gyn, Univ. of Tenn., Memphis, Memphis, TN

Necrotizing enterocolitis (NEC) is a severe intestinal disease which primarily affects infants born early in the last intrauterine trimester. In addition to immaturity, the etiology of NEC includes infection and enteral feeding. Infants who develop NEC as late as 2 to 4 wk of age have been reported to have elevated platelet activating factor (PAF) and xanthine in the first several days of life. Biochemical evidence of early tissue damage followed by later disease suggests that the intestine has subclinical disease for weeks. Clinically significant disease may finally occur after a triggering event which stresses the damaged intestine; e.g., bacterial overgrowth following antibiotic use and/or full enteral feeding. Because of the high mortality associated with NEC, clinical management currently involves delaying full enteral feeding for all $<1.5 \mathrm{~kg}$ infants even though only approximately $20 \%$ are at risk for NEC. Biomedical engineering techniques for assessing intestinal motility could help identify infants with subclinical intestinal disease. If gut motility could be shown to be a sensitive and specific predictor of abnormal gut physiology and could be inexpensively and safely monitored in the first weeks of life, safer approaches to feeding infants born $\langle 1.5 \mathrm{~kg}$ could be devised. Techniques used for monitoring gut function will be reviewed.
287

DEVELOPMENT OF A GRAPHICAL USER INTERFACE TO FACILITATE INTERACTION WITH A PATIENT-SPECIFIC DRUG DOSING PROGRAM

Dean F. Sittig, Ph.D., Zhihe Jiang, M.S., Roger W. Jelliffe*, M.D.

Center for Biomedical Informatics, Vanderbilt University, Nashville, TN

* Laboratory of Applied Pharmacokinetics, University of Southern California, Los Angeles, CA

The user interface is where carbon-based humans, silicon-based computers, and knowledge-based sof tware meet. As any computer-alchemist can tell you, combining such disparate elements along with knowledge (an always elusive quantity) in an attempt to produce gold (in our case the correct drug dose) is difficult if not impossible. The relatively recent development of graphical user interfaces (GUI) is an attcmpt to diminish the blast when these three elements fuse. This presentation will describe the development of a prototype graphical user-interface which is designed to facilitate the process of generating a patient-specific drug dose using bayesian modelling software. Key concepts in the design process are to:

1) Provide feedback to the user immediately following the completion of a task.

2) Make as much relevant information as possible visible on the screen.

3) Enable physicians to enter data quickly and accurately and then to see the effects of changes in various parameters instantaneously.

4) Eliminate the possibility of making errors.

5) Translate the therapy goal directly into an action (in this case a pharmacy order). The graphical user interface was developed in Visual Basic (Microsoft, Inc.) and runs under the Windows 3.1 (Microsoft, Inc.) operating system. All patient demographic data are stored in a relational database (Access, Microsoft, Inc.). The GUI and the database communicate via dynamic data exchange (DDE) links. The presentation will emphasize the interface design process and conclude wilh several of the key lessons leamed.

\section{8}

ESTIMATION AND CONTROL: APPUCATION TO OPTIMIZING CHEMOTHERAPY REGIMENS FOR PEDIATRIC CANCER PATIENTS. John H. Rodman Pharm.D. Pharmaceutical Department, St. Jude Children's Research Hospital and Department of Clinical Pharmacy, Univerisity of Tennessee, Memphis, TN. 38105

A growing number of clinical studies demonstrate that intersubject pharmacokinetic variability for anticancer drugs is substantial, can be correlated with risk of toxicity and, most importantly, has prognostic importance for patient outcome. These results provide the impetus for clinical research to define quantitative relationships for plasma drug concentrations with toxicity and efficacy to guide drug therapy decisions. Recent advances for modeling, estimation and control of pharmacokinetic and pharmacodynamic systems has fostered the development of clinically useful models pharmacodynamic systems has fostered the development of clinically useful models regimens of individual patients during routine therapy. Representative studies with the anticancer drugs carboplatin and teniposide, will be presented to illustrate the application of population data for open loop control strategies based on patient characteristics/pharmacokinetic parameter regression models and Bayesian estimation for adaptive control strategies with limited sampling study designs. For carboplatin, an open loop control reduced intersubject variability in systemic exposure from $48 \%$ to $33 \%$ in 68 prospectively studied patients. For teniposide, an a Bayesian adaptive control strategy increased median dose intensity by $50 \%$ over conventional fixed dose regimens. The estimation and control algorithms have been incorporated into PC based software easily used by clinicians and drug delivery has been facilitated by microprocessor driven programmable infusion devices permitting outpatient therapy. The clinical utility and computational feasability of extending these deterministic control strategies to a stochastic framework is currently under development 
289

\section{AN ALGORITHM FOR X-RAY BONE DENSITOMETRY USING A SMAL NUMBER OF PROJECTIONS \\ Lawrence M. Jordan, Ph.D \\ Department of Biomedical Engineering, University of Tennessee, Memphis}

A method utilizing digital dual-energy subtraction $X$-ray radiography for measuring calcium densities localized to the cortical and cancellous regions of bone cross sections is being developed. The method utilizes a small number of $X$ ray projection images obtained at roughly equal angles in the specimen cross section plane. Use of a variational algorithm for experimental bone shape determination based on a simple model of the mineral density distribution within sectioned cortical and cancellous areas is described. Performance of the algorithm when applied to simulated projection images of phantom bone specimens of various shapes is discussed. It is shown that, in simple cases, a high degree of overlap of estimated and reference bone shapes can be achieved using three projection directions.

290

THEORY ON RADIATION DETECTORS

G. C. Giakos, FA DiBianca and S Devidas

Department of Biomedical Engineering, University of Tennessee, Memphis, TN 38163

This paper presents theoretical aspects on high pressure kinestatic Charge Detectors (KCD). The electron drift velocity, $w$, is given by Christophorou as,

$$
W=-\frac{4 \pi}{3} \frac{e}{m N_{\text {Torx }}} \frac{E}{p} \int_{0}^{\infty} \frac{v^{2}}{\sigma_{m}(v)} \frac{d f_{0}}{d v} d v
$$

where $\sigma_{m}$ is the momentum transfer cross-section and $f_{0}$ is the spherically symmetric term in the expansion of the electron velocity distribution. We may approximate Eq. 1 for a given $E / P$ value as:

$$
w \propto \frac{1}{\langle\sigma\rangle\langle\varepsilon\rangle^{1 / 2}}
$$

where $\langle\sigma\rangle$ is the average overall electron scattering cross section and $\langle\epsilon\rangle^{1 / 2}$ is the square root of the mean electron energy at that $E / P$ value. Polyatomic molecules mixed with a rare gas reduce the electrons energy so that the mixture is more "transparent" to electrons in this range minimizing the chances for an electron attachment or for a large electron dispersion leading to a reduced ionic mobility.

This work was supported by PHS Grants Number CA55378 and AR41376, awarded respectively by the National Cancer Institute and the National Institute for Arthritis, Musculoskeletal and Skin Deseases, DHHS.

291

\section{DUAL-ENERGY IMAGING WITH A KINESTATIC CHARGE DETECTOR}

David C. Spelic, Rohert J. Endorf, Sunil Kulatunga

Dept. of Physics, University of Cincinnati

Frank A. DiBianca, Herb D. Zeman, George C. Giakos

Dept. of Biomedical Engineering, University of Tennessee, Memphis

A method of diagnostic $x$-ray imaging, using the concept of Kinestatic Charge Detection (KCD) is being evaluated for use in dual-energy imaging. Preliminary results of theoretical optimization studies and of detector optimization will be presented. Parameters studied are detector medium, detector geometry, $\mathrm{x}$-ray tube parameters, beam filtration, and the variation of optimal detector configuration with variations in patient size. Theoretical pixel variance and signal-to-noise ratio studies as well as measurements of experimental detector performance show that the KCD can not only provide dual-energy images of good quality, but can easily be adapted to chest as well as mammographic dual-energy applications.
292

X-RAY SOFT TISSUE COMPOSITION MEASUREMENT

H.D. Zeman

Dept. of Biomedical Engineering, University of Tennessee, Memphis

An experimental $X$-ray imaging system using a Kodak Lanex Regular X-ray intensifying screen, a high-speed macro lens, and a liquid-nitrogen-cooled CCD TV camera, has been used to make quantitative bone mineral density measurements in rats. The system produces separate tissue and bone images of the experimental animals. Modeling calculations have shown that the same technique used to separate bone and soft tissue should also be able to separate muscle and fat as long as no bone is present behind the soft tissue areas of interest. The first experimental tests of this techniques are just beginning, and more complete tests will be reported at the conference. The possible applications of separate muscle and fat $\mathrm{X}$-ray images would be to the diagnosis of breas cancer, wasting syndrome in AIDS, muscular dystrophy, cystic fibrosis, and other diseases.

\section{3}

Linearity and Artifact Production in a Kinestatic Charge Detector Charles R. Tenney M.S., Department of Biomedical Engineering, University of North Carolina at Chapel Hill

The Kinestatic Charge Detector (KCD) is an x-ray detector for slot-beam digital radiography based on an ionization chamber in which ion drift is synchronized with detector scanning. Ionization chambers exposed to high radiation levels experience reduced drift velocity due to space charge and loss of signal due to ionic recombination. These effects may be counteracted by increasing the electric field, which increases drift velocities and reduces charge densities. In a KCD, increased drift velocity requires increased detector scan velocity, reducing exposure time at each image point. Accordingly, space charge and recombination effects must be traded off against tube loading for a given exposure. Charge densities in the KCD do not react instantly to changes in spatially nonuniform radiation fields, so a KCD may also show artifacts at edges: recombination will cause the signal to undershoot or overshoot its equilibrium value at an edge, while space charge will cause displacement of signals near the edges. For a uniform radiation field, recombination loss for a given photon's signal depends on the detector location at which it is recombination loss for a given photon's signal depends on the detector location at which it
absorbed. This will broaden the pulse-height distribution and reduce the detector's DQE. These effects are proportional to exposure rate and to the square of exposure time. For a given exposure, they may be minimized by using the highest possible exposure rate for the shortest possible time. The effects also depend on the detection medium employed. For typical beam spectra, a 20 millisecond exposure of $1 \mathrm{mR}$ would cause 4-5\% recombination loss in xenon, which could produce noticeable artifacts. Exposure limits would be about twice as high for krypton. A clean system could reduce electron attachment, as could an electron-cooling additive such as carbon dioxide. The latter, however, would be expected to increase electron-ion recombination rates, and a careful study would be required to assess its total effect. Space charge effects would be reduced by field shaping electrodes at the front of the chamber, especially for denser media in which ions will be closer to the electrodes.

\section{4}

Contrast Resolution of a Kinestatic Charge Detector

Charles R. Tenney M.S., Department of Biomedical Engineering, University of North Carolina at Chapel Hill

The Kinestatic Charge Detector (KCD) is unusual among current or proposed radiographic detectors in that it may be made thick along the axis of the incoming $\mathrm{x}$-ray beam without sacrificing spatial resolution, and with adequate beam utilization. This allows significant increases in QDE and DQE over conventional detectors. As a slot-beam detector, it is capable of excellent scatter rejection without loss of primary photons. Accordingly, the KCD should display a superior ability to image low-contrast objects. Is has been noted by several researchers that the detectability of low-contrast objects in $x$-ray images depends not only on detector DQE and photon fluence (or equivalently, on in $x$-ray images depends not only on detector $D Q E$ and photon fluence (or equivalently, on
NEQ) but also on the modulation of the beam spectrum in the energy domain by the objects $\mathrm{NEQ}$ ) but also on the modulation of the beam spectrum in the energy domain by the objects
which are radiographed, coupled with the detector's spectral response. Although the basic which are radiographed, coupled with the detector's spectral response. Although the basic processes by which this occurs are well understood, a simple, unified explanation of these
effects has so far been lacking. Tapovaara and Wagner (Phys. Med. Biol. 30 p. 519, 1985) effects has so far been lacking. Tapovaara
have provided a good start in this direction.

The expressions of Tapiovaara and Wagner are shown to yield simple, physically meaningful indices of image quality and detector efficiency. These and similar indices are used to formulate a rigorous definition of contrast resolution in terms of material properties of the radiographed object and its background. This definition accounts for photon statistics, detector efficiency, object antenuation, and detector spectral response.

The contrast resolution achievable with xenon and kypton KCD devices is evaluated for a variety of detector configurations and bearn spectra. Contrast resolution for some conventional detectors is evaluated for comparison. DQE and detected contrast are examined by analytic and Monte Carlo simulations, in the limits of zero and high spatial frequency. Scatter effects and scatrer rejection are examined by Monte Carlo modeling. Together, these results give the contrast resolution effects of all $x$-ray processes for $K C D$ and other detectors. 
295

ASSESSMENT OF SYSTEM RELATED ERRORS IN QUANTITATIVE PHASE CONTRAST MAGNETIC RESONANCE IMAGING

Guarnieri S, MS; Kirsch JE, Ph.D.

Center for BME, University of KY, MRISC Dept, University of KY.

Velocity encoded phase contrast magnetic resonance imaging is sensitive to small alterations in phase due to motion and magnetic field errors. Decreasing system related phase errors increases the accuracy and useful quantitative information of the acquisition. In this study, phase offsets and time dependent phase shifts were observed for both static and constant velocity phantoms. For errors found in static phantoms, $60 \mathrm{~Hz}$ power oscillation in $\mathrm{RF}$ transmission and evolving eddy current fields were isolated for their contributions. Power oscillation in the RF transmission can cause phase offsets of $1 \%$ between two velocity nulled echoes regardless of the encoding range. The effect was decreased to $<0.1 \%$ by changing the power oscillation compensation adjustment and pulse sequence timing. Errors from evolving eddy current fields were reduced by allowing multiple echoes and altering maximum pulse amplitude. For constant velocity plug flow, errors were associated with shim offsets and gradient calibration. Misadjusted shim fields produce phase offsets which do not always cancel digitally. Although gradient calibration does not affect the phase nulling of a particular sequence, it will produce velocity encoding errors that vary linearly with gradient strength. Many system related phase errors can be reduced to allow a more accurate quantitative measurement of velocity related phase.

296

SEGMENTATION OF MAGNETIC RESONANCE IMAGES WITH A

SELF.ORGANIZING MAP

R. Deaton, T.C. Edwards, K. M. Haarmon, and H. Kakde

Department of Electrical Engineering, Memphis State University, Memphis, TN 38152 W. E. Reddick

Diagnostic Imaging Lab, St. Jude Children's Research Hospital, Memphis, TN 38101

A self-organizing map is used to segment a set of MR images of the brain. The set of $M R$ images consists of proton density. T2-weighted, and T1-weighted.

Corresponding pixel intensities from each image are combined in an input vector of

length three. Through an iterative process, the self-organizing map separates the set of input vectors into classes. The self-organizing map is a type of neural network. For this work, the network was a four-by-four Kohonen slab. The weight vectors of the processing units or neurons that were closest to the current input vector, where distance is measured with the Euclidean metric, were adjusted to match the input vector. Not only the closest weight vector was adjusted, but also its neighbors, thus preserving the topology or neighborhood relations of the input space. The weight adjustment of the self-organizing map was done on a portion of the three images. After training, the entire set of three images was presented to the self-organizing map. In the winner-take-all strategy of the map, the neuron whose weight vector most closely matched the input vector for a given pixel was declared the winner and its designation assigned to that pixel. The classification, which was similar to regions of different tissue type, produced a good segmentation of the image. Borders or edges between regions were assigned to different neuron bins than the surrounding tissue. Since the mapping is a point operation, smoothing and broadening of edges was not a problem, as it is for area techniques. This should be useful for volume estimation and reconstruction from MR images.

297

CONVERSION OF THE STEADY STATE FREE PRECESSION (SSFP) SIGNAL INTENSITY TO CONCENTRATION

Hilaire L, M.S.; Kirsch JE, Ph.D.

University of KY, Center for BME; University of KY, MRISC dept.

The conversion of the magnetic resonance imaging (MRI) signal intensity to concentration is a necessary requirement for obtaining accurate quantitative perfusion information. The ability of the steady state free precession (SSFP) technique to meet this requirement using the ratio of the echo and FID signals was examined. Phantom experiments, collected with a 1.5 Tesla whole body imager, were used to analyze the accuracy of converting the SSFP signal intensity to concentration. The SSFP technique parameters were: $T R=12.6 \mathrm{~ms}$, $T E_{F I D}=4 \mathrm{~ms}, T E_{\text {echo }}=21.6 \mathrm{~ms}, F A=90 \circ$, temporal resolution $=806 \mathrm{~ms}, F O V=94 \times 125 \mathrm{~mm}, M A=48 \times 64(1.95 \times 1.95 \mathrm{~mm})$, total scan time $=51.6$ secs $(64 \times 2$ images). Contrast media (Gd-DTPA, 0.1 $\mathrm{mM}$ to $5.0 \mathrm{mM}$; AMI-227, $0.018 \mathrm{mM}$ to $0.537 \mathrm{mM}$ ) in $30 \mathrm{ml}$ samples were studied. Region of interest measurements were made and data was post-processed to yield calculated concentration with SSFP technique versus measured concentration of samples. The results obtained with the SSFP technique demonstrate the possibility of acquiring accurate conversion of signal intensity to concentration in a perfusion experiment. Conversion of signal intensity to concentration of the individual signals specially the FID results in erroneous information evidenced as negative concentration values. These findings are also consistent in preliminary first pass studies of the normal cat brain.
298

CONTRIBUTIONS OF SUSCEPTIBILITY AND RELAXIVITY EFFECTS IN PERFUSION IMAGING

Hilaire L, M.S.; Kirsch JE, Ph.D.

University of $\mathrm{KY}$, Center for BME; University of KY, MRISC dept.

The role of relative contributions of susceptibility and relaxivity in perfusion imaging was examined. Specifically their effect on the signal intensity of the steady state free precession (SSFP) technique was analyzed. Phantom studies, collected with a 1.5 Tesla whole body imager, were used to characterize relaxivity (R2) and susceptibility ( $x)$ constants of Gd-DTPA $(0.1 \mathrm{mM}$ to $5.0 \mathrm{mM})$ and AMI-227 $(0.018 \mathrm{mM}$ to $0.537 \mathrm{mM}$ ) with established methods. A molar susceptibility value $\left(2.06 \times 10^{-1} \mathrm{ml} / \mathrm{mol}\right)$ and a relaxivity constant $\left(75.854 \mathrm{mM}^{-1} \mathrm{~s}^{-1}\right)$ were obtained for the superparamagnetic agent AMI-227. A lower molar susceptibility value $\left(2.86 \times 10^{-2} \mathrm{ml} / \mathrm{mole}\right)$ and relaxivity constant $(5.04$ $\mathrm{m} \mathrm{M}^{-1} \mathrm{~s}^{-1}$ ) were determined for the paramagnetic agent Gd-DTPA. Results are in good agreement with published data. Computation of R2* based on the individual SSFP echo and FID signals yield false results due to $\mathrm{T} 1$ contribution. However, $\mathrm{R} 2 *$ calculated with the ratio of the SSFP echo and FID signals were consistent showing greater values for AMI-227 $\left(97.534 \mathrm{mM}^{-1} \mathrm{~s}^{-1}\right)$ than Gd-DTPA $\left(7.739 \mathrm{mM}^{-1} \mathrm{~s}^{-1}\right)$, due to the elimination of longitudinal relaxation ( $\mathrm{T} 1$ ) influence confirmed by previous work. These results suggest that although the degree of susceptibility and relaxivity contributions varies with contrast agents, any contrast media can be used in perfusion imaging when the ratio of the SSFP signals is used.

299

TOWARD ADAPTIVE FILTRATION OF 2D TIME-OF-FLIGHT MRA DATA Issa Y. Khoury, Elizabeth Abrahams, P. J. Keller and B. P. Draye Dept. of MRI Research, BNI, St. Joseph's Hospital, Phoenix, AZ

Magnetic resonance angiography (MRA) is often accomplished by the acquisition of $3 D$ image data. An alternative scheme involves the acquisition of $2 \mathrm{D}$ slices and building a volume image. This alternative offers the bighest possible refreshment of flowing spins in all regions of the image volume at the expense of increased minimun $T E$ and voxel size. However, each of these two acquisition modes has strengths and weaknesses.

In NMR spectroscopy it is routine to apply apodization to the data before Fourier transformation to increase the signal-to-noise ratio. The most commonly used apodization function is a "matched" filter which matches the exponential decay for the data. All three axes are filtered in $3 D$ images, while only two axes are filtered in $2 D$ acquisition schemes.

When viewing a projection of 2D time-of-flight data in the slice axis to see the entire length of the vessels, the vessels seem to have a ragged or molted appearance. The current work involves the application of the same apodization process in the slice dimension of 2D data as is routine for 3D data to eliminate the ragged appearance of the vessels. This is accomplished by performing an iFFT in the slice direction of the raw data, measuring the exponential decay in the slice axes, creation and application of a matched filter then performing a FFT in the slice dimension. After this, the usual 2D reconstruction is performed. It might only be necessary to perform the iFFT on one line of data in the slice dimension to measure the decay constant.

300

SIGNAL PROCESSING OF DYNAMIC CONTRAST-ENHANCED MR IMAGES W.E. Reddick, R.G. Steen, and J.S. Taylor

St. Jude Children's Research Hospital, Department of Diagnostic Imaging

Dynamic contrast-nhanced MR imaging (DEMRI) is under study to determine ito ability to reveal the perfusion characteristics of tumors. A series of 55 $T_{1}$-weighted MR images were acquired on a $1.5 \mathrm{~T}$ imager and transferred to an offline workstation. Five baseline images were acquired prior to a bolus injection of $\mathrm{Gd}$ DTPA contrast agent and another 50 were acquired post-injection. Each image was calculates the rate of contrast accumulation, the rate of contrast washout, and the amount of contrast accumulated relative to the sigral intensity.

The DEMRI processing calculates the derivative of the signal intensity with respect to time using a piece-wise linear fit on a three point moving window. The initial uptake region of the signal intensity curve is sigmoid in shape with a maximum derivative occurring at the inflection point. The maximum derivative is determined and a search is performed to find the first point before and after the maximum that has a derivative les than ten percent of the maximum. These two points define the uptake interval. The washout interval is defined as a 60 second interval starting 90 seconds after the bolus first enters. The rate for each of these intervals is determined as the slope of a linear least-squares fit. The relative amount of contrast sccumulated is determined by subtracting the minimum and maximum signal intensities occurring in the time series. The three parameters are assigned grey scale values to creste three images and transferred back to the imager for viewing and filming. An institutional protocol is evaluating the significance of this analysis for assessment of response to chemotherapy in bone and soft tissue tumors in children. 
301

PRINCIPLES OF THE WAVE PERMITTIVITY AND WAVE PERMEABILITY George C. Giakos

Department of Biomedical Engineering, University of Tennessee, Memphis, TN 38163

Microwave dosimetry deals with microwave energy interaction with biosystems. According to the author's theory [1], when a pulsed, modulated microwave signal impinges on a structure more or less smaller than the carrier wavelength, the "wave permittivity" concept may be associated with transient events, occurring during a wavefront rearrangement (quasi resonance). The idea is to express the wave permittivity as a variant scalar, in terms of propagating and stored field energy density parts, treated as components of the four-tensor of electromagnetic momentum:

$$
\epsilon_{i j}=\frac{S_{i j}}{B \cdot B}
$$

. Eq. [1] enlightens completely Eq. 2 [2] which was previously and independently mentioned by Brillouin for dielectric media:

$$
W \cos ^{2} \theta v_{p h}=W v_{g r}
$$

where $v_{p h}$, is redefined as the velocity of the signal $c / \cos \theta$ (translational motion) with which the excess of field energy density propagates [1]-[2], and $\theta$ is function of the energy balance of the guiding structure. When non stored terms are involved in the energy balance of the system, the wave permittivity of waves emerging from an isotropic source is equal to $\varepsilon_{0}$. Similarly, a wave permeability concept can be defined [1] G.C. Giakos, "Foundations of the Wave Permittivity Diversity Imaging", Progress in Electromagnetic Research Symposium, Proc. PIERS 1994 pp. XX, Jet Propulsion Laboratory, California Institute of Technology, July 1993.

[2] G.C. Giakos,"Detection of Non-TEM Waves in Open Media", Ph.D Dissert., 1991.

302

$$
\begin{gathered}
\text { MODERN MICROWAVE IMAGING TECHNIQUES } \\
\text { C. Giakos, T.K. Ishiit and F.A DiBianca } \\
\text { Department of Biomedical Engineering } \\
\text { University of Tennessee, Memphis, TN } 38163 \\
\text { + Department of Electrical and Computer Engineering } \\
\text { Marquette University, Milwaukee, W } 53233
\end{gathered}
$$

The purpose of this work is to provide a review of the current microwave techniques as applied to medical imaging. It has been suggested that microwaves can be profitably ufilized in detecting soft tissue abnormalities providing a clear view of the functional state of the living structure. The authors will introduce short theoretical aspects characterizing the interaction of electromagnetic waves with complex dielectric media. Following this, an overview of the techniques of bioimage formation based on microwave principles is offered. These techniques can be passive or active. Passive imaging techniques such as microwave thermography rely on the radiometric measurements of thermal emissions. On the other hand, active methods utilize microwave sources to interrogate biological structures. A variety of active techniques involves measurements of physical parameters such as putse dispersion, conductivity, polarization and scattering parameters. Although the spatial resolution of a microwave imaging system is poor, an extremely high contrast resolution can be achieved.

303

HIGH FAEQUENCY COILS FOR CUNICAL NMR IMAGING AND SPECTROSCOPY JT Vaughan, HP Hetherington, JG Harrison, PJ Noa, GM Pohost The University of Alabama at Birmingham

In the pursuit of improved signal-to-noise ratios in nuclear magnetic resonance (NMR) detection for clinical imaging and spectroscopy, higher frequency detectors (coils) must be developed for use with the higher $B_{0}$ magnet fields required. Radiofrequency coil design for human studies at 4 Tesla and higher requires new methods. As coil dimensions become large fractions of wavelengths, conventional lumped element designs sultable for lower fields must give way to distributed line and cavity designs to preserve performance at higher frequencies. The reactive quarter wave transmission line element and resonant cavity of the transverse electromagnetic (TEM) circuit are recommended to replace the inductor $\mathrm{L}$, and capacitor $\mathrm{C}$, of the lumped element (LC) circuit where possible for coll designs above $100 \mathrm{MHz}$. Lumped elements can facilitate practical tuning, matching and dimensional adjustments in TEM-LC "hybrid" coils. The coil's B, radiofrequency magnetic field distribution in the human body can be very different than the free space field, therefore th: $B_{1}$ time-dependent fields for the coil and human load must be considered in the design process. Predictions of $B_{1}$ field distributions in human anatomy for high frequency surface and volume coils are made by numerically solving Maxwell's equations for finite element coil-tissue models. Coil current distributions are thereby adjusted for optimum field homogeneity and efficiency for an anatomic region of interest.

The purpose of this paper is to report coils designed accordingly for surface and volume applications at the highest fields currently available for clinical applications. Performance specifications and results from prototype high frequency TEM coils will be discussed and compared to theoretical predictions. The inherent signal-to-noise advantage of high field NMR is realized in clinical images and spectra produced with properly designed high frequency coils.
304

LOW FREQUENCY CURRENT DENSITY IMAGING IN RABBITS.

M.L.G. Joy ${ }^{\ddagger}$, V.P. Lebedev ${ }^{*}$, J. Gatti ${ }^{\ddagger}$, R. M. Henkelman ${ }^{\dagger}$ Institute of Biomedical Engineering ${ }^{\ddagger}$ and Department of Medical Physics ${ }^{\dagger}$ University of Toronto, Canada. Pavlov Institute for Physiology*, St. Petersburg, Russia.

Low Frequency Current Density Imaging (LF CDI) is a recent $[1,3,4]$ imaging modality that produces images of electric current density which arises from external electric sources. The LF CDI techmique uses a Magnetic Resonance Imager to measure the magnetic flux density, $\mathbf{B}$, which is produced by the current density, $\mathbf{J}=\frac{\nabla \times \mathbf{B}}{\Delta \mathbf{0}}$. Electroanalgesia produced by applying electric currents to the brain has been reported[2]. To better understand how this analgesia might arise, images of current fowing in the brains of rabbits using two electrode placements, one effective and one ineffective, were made. Analysis of these images shows localized current pathways and a distinct difference due to electrode placement.

\section{References}

[1] M. Joy, G. Scott, and R. Henkelman. In Vivo Detection of Applied Electric Currents by Magnetic Resonance Imaging. Magnetic Resonance Imaging, 7:89-94, 1989.

[2] V. P. Lebedev, Y. S. Katznelson, V. A. Leosko, O. B. Illyinski, A. B. Savchenko, and A. V. Malygin. Selective electrostimulation of deep brain stem opioid structures through the surface head electrodes in animals and humans. In Proceedings of the First World Congress For Electricity and Magnetism in Biology and Medicine. San Francisco Press, 1992.

[3] G. Scott, M. Joy, R. Armstrong, and R. Henkelman. Measurement of Non-Uniform Current Density by Magnetic Resonance. IEEE Trans. Med. Imaging, 10(3):362-374, Sept 1991 .

[a] G. Scott, M. Joy, R. Armstrong, and R. Henkeiman. Sensitivity of Magnetic Resonance Current Density Imaging. J. Mag. Res., 97, 1992

305

NOVEL ENGINEERING APPROACHES TO BIOMEDICAL MAGNETIC RESONANCE

Joseph J.H. Ackerman

Department of Chemistry, washington University, st. Louis, MO 63130-4899

Biomedical magnetic resonance, both imaging and spectroscopy, presents a fertile ground for the development of new engineering strategies. Three novel developments will be described. (1) Bayesian probability theory provides a powerful, rigorous framework for optimal estimation of the parameters that characterize the time-domain signal. The use of Bayesian analysis for NMR signal detection, model selection and parameter estimation will be presented. (2) The electron spin represents a dipole 660 times as large as that of ${ }^{1}$; yet, because of ultra short relaxation times and microwave skin depth related penetration problems, it has not found widespread use as an in vivo magnetic resonance label. A spectrometer design providing essentially zero dead time and an absence of rf excitation (!) will be offered as a solution to these issues. (3) spectroscopy in vivo is often made difficult by poor signal-to-noise and complex gradient based pulsed sequences. A simple, robust, high sensitivity approach to localized spectroscopy via surface-spoiling gradients will be discussed.

306

A PEDIATRIC BODY COIL

RJ. Ogg, J.M. Andrew, and J.M. Langston

St Jude Children's Research Hospital, Memphis, Tennessee

The radio-frequency (RF) coil is an important component in determining the signal-tonoise ratio (SNR) that can be achieved in nuclear magnetic resonance (MR) experiments. High quality components, careful fabrication, and appropriate geometry are essential for optimal signal detection (1). The RF coils provided with clinical MR instruments, designed to accommodate adult patients, are too large for many pediatric patients. The resulting loss in SNR can be a significant problem in pediatric oncology patients, where visualization of metastatic disease, tumor recurrence, or response to therapy requires optimal image quality. We have built a pediatric body coil, intermediate in size between the standard head and body coils, to improve SNR in body imaging of pediatric oncology patients.

The coil is a cylindrical four ring quadrature birdcage $(2,3)$ with sixteen elements: diameter $=37 \mathrm{~cm}$, inner structure length $=18.5 \mathrm{~cm}$, outer structure length $=9.25 \mathrm{~cm}$, and the uncoupled unloaded coil $Q=500$. With inductive coupling and impedance matching to $50 \Omega$, unloaded $Q=$ 240 . Images of a spherical phantom (radius $=12.5 \mathrm{~cm}$ ) were acquired at $1.5 \mathrm{~T}$ (Siemens Magnetom SP) using the pediatric body coil (loaded $Q=168$ ) and the standard body coil. Image uniformity was comparable with the two coils; SNR was 1.9 times higher with the pediatric coil than with the standard body coil. Matching coil impedance, while maintaining quadrature isolation and current balance in the two outer structures, is difficult for very low $Q(-10)$. We will describe the coupling optimization and a novcl patient bandling system, and present MR images demonstrating improved SNR for pediatric body imaging.

1. Hoult, D.I. and Lauterbur, P.C., J. Magn. Reson., 34, 425-433, 1979.

2. Murphy-Boesch, J., Srinivasan, R., and Carvajal, L., SMRM Abstracts, 125, 1991

3. Zur, Y., Gotshal, U., and Grach, Y., SMRM Abstracts, 271, 1992. 
307

QUANTITATIVE INTERPRETATION OF SINGLE-VOXEL MR SPECTRA R. Wilcott, W. Riddle, K. Cecil, and J. Gibbs

Vanderbilt University, Nashville, TN

Development of single-voxel gradient-localized MR spectroscopy of the human brain is approaching clinical utility, but its acceptance requires verification of its accuracy and precision. A standardized aqueous solution was prepared containing the major constituents reported from MR spectra of the brain: $\mathrm{N}$-acetyl aspartate, creatine, and choline, each 45.0 millimolar in methyl protons. A solution of each constituent was also prepared. NMR spectra were obtained at $9.2 \mathrm{~T}(400 \mathrm{MHz})$, using a Bruker AM 400 spectrometer. Single-voxel MR spectra were obtained at $1.5 \mathrm{~T}(64 \mathrm{MHz})$, using a Siemens Magnetom SP and a spin echo sequence at $T E=135 \mathrm{~ms}$. Accuracy of the method was assessed by measurement of intra- and inter-molecular peak intensity ratios. Precision was determined by repetitive measurements. Both were within $\pm 5 \%$ for all solutions at $400 \mathrm{MHz}$. At $1.5 \mathrm{~T}$, precision was generally within $\pm 10 \%$. However, using clinical protocols (TR $=1600 \mathrm{~ms}$ ), peak intensity ratios for the singlet signals varied from expectation by as much as $50 \%$. These deviations were brought within $10 \%$ with very long TR, which required clinically unacceptable measurement times. Sources of error that we considered arose from the spin system imodulation by T1, T2, J coupling, and chemical shift), the hardware (field homogeneity imperfections in RF pulses or gradient linearity, and effect of eddy currents and their compensation), and the operator (inconsistency in performance of spectroscopy and subjectivity during data reduction, both intra- and inter-examiner). Clinically acceptable precision may be achieved in single-voxel MR spectroscopy by carefu technique. However, inaccuracies may arise from several sources, some under operator control and some not. Small differences in peak intensities or intensity ratios seen in diseased states should be interpreted conservatively.

\section{8}

CLASSIFICATION AND MONITORING OF MUSCLE DISEASES USING ARTIFICIAL NEURAL NETWORKS AND P-31 MR SPECTROSCOPY S. Kari, T. Vital, and J.H. Park.

Vanderbilt University Medical School, Nashville, TN

Dermatomyositis (DM) is an inflammatory muscle disease characterized by extreme muscle weakness and a typical erythematous rash. A new classification of this disease, namely, amyopathic DM presents with a severe rash but no clinical muscle weakness. An artificial neural network (ANN) has been developed for the differentiation of patients with myopathic or amyopathic DM. The P-31 MR spectra of the thigh muscles, which were acquired during rest with a 1.5-T Siemens Magnetom, provided the input features to train the ANN using the back-propagation algorithm. The features extracted were Pi, PCr, ATP, PDE, total Phosphate and ratio subsets. The network output was the classification of normal controls, myopathic and amyopathic DM. The ANN was completely successful in differentiating the three groups. In addition, the ANN detected a serious disease relapse as a patient regressed from an anyopathic to myopathic condition. In conclusion, this study suggests that ANN may be of use in the classification and continuing clinical evaluation of chronic muscle diseases.

309

IN VIVO ${ }^{19} \mathrm{~F}$ AND ${ }^{7} \mathrm{LI}$ NMR OF PSYCHOACTIVE DRUGS IN HUMANS

R.A.Komoroski, J.E.O.Newton, D.Cardwell, J.Sprigg, C.N.Karson

Depts of Radiology, Pathology, and Psychiatry, University of Arkansas for Medical Sciences, and Meclellan VA Hospital, Little Rock, AR.

In vivo ${ }^{19} \mathrm{~F}$ NMR spectroscopy has been used to detect fluorine-containing psychoactive drugs in human brain. A quadrature birdcage coil provided sufficient signal-to-noise ratio for quantitation, measurement of spin-lattice relaxation times, and crude iocalization of the antidepressant fluoxetine. The weak ${ }^{19} \mathrm{~F}$ signal from the antipsychotic drug trifluoperazine could also be detected. Similar results were obtained for ${ }^{7}{ }_{L i}$ NMR of lithium, a drug used to treat manic-depressive illness, in human brain. The higher brain concentration of $L i$ relative to the fluorine-containing drugs permitted faster data-acquisition times and better spatial

localization of the NMR signal. The pharmacokinetics of $\mathrm{L} i$ uptake and elimination were studied.
310

MRI AND P-31 MAGNETIC RESONANCE SPECTROSCOPY (MRS) FOR MONITORING THERAPEUTIC RESPONSES IN MUSCLE DISEASES, J.H. Park, S. Kari, T. Vital, N. Ryder and N.J. Olsen Vanderbilt Medical school, Nashville, TN 37232

Dermatomyositis is an inflammatory, autoimune muscle disease involving severe proximal muscle weakness and rash. The mainstays of treatment are prednisone and immunosuppressive drugs, methotrexate and azathioprine. objective analysis of disease status is important because these compounds have serious side effects and frequent adjustments in dosage are required. Four juvenile and 8 adult patients were evaluated with MRI/MRS during therapy over periods from 4 to 41 months. Using a 1.5-T Siemens Magnetom, inflammation in the diseased thigh muscles was visually analyzed on T2-weighted images and quantified by $T 1$ and $T 2$ values. Inflammation disappeared in 3 to 21 months. P-31 spectra of the quadricep muscles were obtained during rest and exercise. With treatment, the initially elevated $\mathrm{Pi} / \mathrm{PCr}$ ratios were lowered, indicating more efficient generation of PCr and ATP. Additionally, PCr and ATP levels rose consistent with improved strength. In summary, MRI and MRS provided unique quantitative data for evaluation and long-term management of juvenile and adult dermatomyositis.

311

INFORMED CHOICES: OPTIMTZING ${ }^{1} H$ MRS FOR PEDIATRIC TUMOR PATIENTS J.S. Taylor, P.B. Kingsley, R.J. Ogg, W.E. Reddick, and J.W. Langston, St. Jude Children's Research Hospital and U. TN Medical Center, Memphis, TN

One goal of our pilot study on the feasibility and utility of ${ }^{1} \mathrm{H}$ MRS in the management of children with brain tumors was to identify and reduce or correct for the major sources of variance, so that clinical MRS data from multiple sites can be reliably acquired in patient examinations and evaluated as a group clinical study. We developed procedures for assessing errors associated with water suppression, eddy currents and coupled spins, and incorporated these results into quality assurance (QA), acquisition sequences, exam-time calibrations and automated data processing for clinical single-voxel localized, water-suppressed ${ }^{1}$ H MRS. Analyses included Bloch-equation simulations of water suppression ${ }^{1}$ and product operator descriptions of localization sequences ${ }^{2}$, as well as calibrations of instrumental drift and variation. These led to optimized MRS sequences which require fewer time-consuming and error-prone operator adjustments, are less sensitive to $B_{1}$ variations and yield more uniform water suppression. The resulting consistent, high-quality data are easily analyzed by automatic processing algorithms which incorporate prior knowledge of approximate peak regions. Initial analysis of automated processing on a test set of volunteer data showed reduced variance for metabolite peak area ratios derived from the automated analysis of magnitude data compared to ratios obtained by a partially-automated method requiring manual phasing and choice of peak regions. A PC database application was designed to store and facilitate analysis of clinical MRS data, which is by nature very different from the normal image output of the $M R$ imager. The optimized sequences and automated processing are now used routinely for clinical data acquisition and analysis, and are robust. Conclusion: Attention to $Q A$, examination-specific optimization, and automaied data processing can reduce the experimental components of the typically large variance observed for MRS studies.

1. R.J. Ogg, P. Kingsley \& J. Taylor, 34th Expt NMR Conference, Abstract 191, 1993.

2. P.B. Kingsley, 34th Expt NMR Conference, Abstract 177, 1993

312

\section{RELIABIITY OF CAROTID ARTERY LUMEN SIZES DETERMINED BY IMAG \\ PROCESSING OF MAGNETIC RESONANCE ANGIOGRAMS AND IMAGES}

Radiology, Biomedical Engineering, Cardiology, University of Vinginia, Charlottesville, VA

Magnetic resonance imaging (MRI) offers the potential of visualizing and measuring both arterial lumea and wall. Magnetic resonance angiography (MRA) is an established and often used technique, although its predecessor, $x$-ray angiography is the most trusted currently used method. These lumen measuring angiographic methods, bowever, suffer from their inability to examine the arterial wall. Studjes indicate that in the early stages of atherosclerosis, arteries compensate for wall thickening and maintain a constant lumen size. Thus angiographic techniques are unable to examine the early stages of atherosclerosis. The purpose of this study was to determine the repeatability of Jumen size measurements determined from MRI and MRA image data sets, and to determine the accuracy of MRI results using MRA as a standard.

In this initial stage of an ongoing project to quantify atherosclerosis, we determined the reproducibility of measurements of lumen size obtained from a dark-blood T1 weighted MRI exam and a bright-blood MRA exam. MRA exams incorporated TONE pulses and magnetization transfer suppression. Messurements were performed six times each on two normal volunteers and on two patients with known athemscleposis on a Siemens 1.5 Tesla MR imager. Lumen volumes were calculated using a semi-antomated active surface computer processing segmentation technique developed in-house. Lumen areas were determined for left and right common, internal, and external carotid arteries.

We found that MRI and MRA are roughly comparable in their reliability: MRA has a 10\% normalized standard deviation, while that of MRI is $15 \%$. This suggests that both techniques are potentially useful for following atherosclerosis progression/regression. 
313

A COMPARISON OF MEASUREMENTS BY A NEW METHOD OF BONE MINERAL
DENSITOMETRY TOREFERENCE DATA
Peter G. Davis and Lawrence M. Jordan, Ph.D
Department of Biomedical Engineering, University of Tennessee, Memphis

Digital images of an intact rat femur, tissue and bone, embedded in an acrylic casing were obtained with two distinct $X$-ray energy spectra, using a CCD camera, at several different beam angles perpendicular to the bone axis. The rat femur was then sectioned into $1 \mathrm{~mm}$ slices which were separately imaged parallel to the bone axis with the same apparatus to provide the reference data. The method of dual-energy image subtraction was utilized to obtain densitometric data for the intact femur. A variational algorithm[1] was applied for measuring calcium densities localized to the cortical and trabecular regions of sample bone cross sections. The algorithm is based on a simple model of mineral density distributions in cortical and trabecular bone. It is shown that absolute cortical and trabecular mineral content can be measured to moderate accuracy by this method for cases of simple bone geometry.

[1] L. Jordan. "An algorithm for $X$-ray bone densitometry using a small number of projections." private communication, 1993.

\section{4}

\section{FRACTAL MODELLING OF THE DIELECTRIC PROPERTIES OF BONE} D. Andersen

Electrical Engineering Department. Bucknell University, Lewisburg, PA 17837

The dielectric properties (conductivity and permittivity) of cortical bone exhibit a power-law dependence on frequency. This power-law dependence was simulated over the frequency range of $100 \mathrm{~Hz}$ to $10 \mathrm{MHz}$ using a self-similar network of capacitors and resistors. The resistance and capacitance of each branch of the network scaled by a constant factor from that of the previous branch. Such a model reflects the wide range of length scales over which relaxation processes occur in bone. A network with a self-similar topology, but with elements scaled according to the anatomical structures in cortical bone and not by a constant factor was not successful in simulating Pric properties of bone.

Previous models have predicted the dc.conductivity from the number and orientation of vessels in bone. The present model addresses the frequency dependence of the dielectric properties. The modelling of the dielectric properties of bone is useful in understanding the transport of charge mechanisms. such as those involved with the electrical stimulation of bone growth.
316

\author{
SPATIAL AND TEMPORAL RATE OF BONE DEMINERALIZATION DUE TO NON- \\ WEIGHTBEARING AND THE EFFECT OF EXCERCISE \\ Dona M.K., H.D.Zeman,PhD, D.Thomson,PhD \\ Department of Biomedical Engineering, University of Tennessee, Memphis
}

It has been determined that the principal extrinsic influences of loss of bone mass and remodeling are those derived from mechanical loading. This study proposes to determine the validity of that statement and the potential interrelationship between the quality and quantity of physical activity and its role in the prevention/curing of osteoporosis, using rats as a model. The information obtained using this method is more precise, since the bone density variation of the same animal is noted throughout the non-weightbearing period. The influences of weightbearing forces on the bone mass and remodelling are examined using a liquid nitrogen cooled CCD TV camera system from Astromed CCD Inc. th order to measure changes in bone mineral density accurately in a bone which is $-35 \mathrm{~mm}$ long and $2.5 \mathrm{~mm}$ wide (rat's fermur at its narrowest point), a high spatial resolution is required along with high quantum efficiency to reduce $x$-ray quantum noise. The CCD system used has $384 \times 578$ pixels, each of which is $0.022 \times 0.022 \mathrm{~mm}$ in size. The entire array is read out to 16-bits accuracy in about 6 secs. With the liquid $\mathrm{N}_{2}$ cooling, dark current of the CCD is reduced to an immeasurable 1 electron per pixel per hour. The lens opening and magnification setting has also been optimized to achieve good spatial resolution.

317

VIBRATIONAL RESPONSE ANALYSIS OF EWE VERTEBRAL CANCELLOUS BONE PS Weinhold, CF Abrams Jr, SC Roe, JA Gilbert Department of Biological and Agricultural Engineering Department of Companion Animal and Special Species Medicine (Roe) North Carolina State University

Department of Agricultural and Biological Engineering (Gilbert) Mississippi State University

Osteoporosis produces structural changes in vertebral cancellous bone (VCB) that have a directional affect on its mechanical properties. The objective of this study was to evaluate non-destructive vibrational response analysis (VRA) for dynamically assessing the directional elastic moduli of VCB. Orthogonal elastic moduli determined by destructive compression testing were compared to values estimated by VRA for cubes of ewe VCB. Two cubes cranial/caudal to each other were dissected from the lumbar vertebrae of each of six ewes (2-11 years of age). The dynamic elastic moduli of all cubes were assessed by VRA testing in each of the 3 anatomical directions: cranial/caudal (CC), medial/lateral (ML), and dorsal/ventral (DV). The static elastic modulus and compressive strength were determined for each pair of cubes in the $\mathrm{CC}$ and ML directions by compression to failure testing. The moduli and strengths were significantly greater $(P<.05)$ along the longitudinal spinal axis (CC) as compared to the transverse directions (ML and DV), as has been reported for human bone. The mean dynamic and static elastic moduli in a given anatomical direction did human bone. The mean dynamic and static elastic moduli in a given anatomical direction did
not differ $(\mathbf{P}<.05)$. A relatively high correlation was found between the dymamic elastic modulus and either the static elastic modulus $(r=.876)$ or compressive strength $(r=.867)$ These resuits suggest that vibrational response analysis may be an efficient and reliable method of non-destructively assessing the mechanical anisotropy of vertebral cancellous bone.
315

THE USE OF VIBRATION ANALYSIS TO EVALUATE THE INFLUENCE OF DIETARY CHANGES ON THE MECHANICAL PROPERTIES OF WHOLE BONE JB O'Brien, CF Abrams Jr, K Wedekind

Department of Biological \& Agricultural Engineering

North Carolina State University

Mark Morris Associates (Wedekind)

The objective of this research is to evaluate vibration response analysis as means of estimating the mechauical properties of whole bones relative to dietary effects. Three-poin bending is a commonly-used method which should be limited to straight specimens having a symmetrical cross section and a length-to-diameter ratio of at least 10; whole bone infrequently meets these constraints. Machined test specimens may be used, but the time required and artifacts of machining are probelms. Vibration response analysis is an alternative method that is non-destructive and relatively fast. Standards call for a length to diameter ratio of 5 to 20 ; this wider range allows for a variety of bone shapes. Specimens with a ratio below 5 or greater than 20 may be difficult to excite in the fundamental mode The bones for this research were part of a study to determine if supplemental zinc produces measurable differences in the mechanical properties of bone. An experiment was conducted with swine to characterize the effects of supplemental $\mathbf{Z n}$ on growth performance, bone $\mathbf{Z n}$ plasma Z,n, and the mechanical properties of bone. In the experiment, 192 pigs were fed corn-soybean meal diets supplemented with feed-grade ZnSO4.H20 to provide $0,5,10,20$, 40 , and $80 \mathrm{mg} / \mathrm{kg}$ supplemental $\mathrm{Zn}$. The third and fourth metacarpal bones were tested using both vibration analysis and three-point bending to evaluate the effect of increasing $\mathrm{Zn}$ levels on bone. 
319

\section{$E_{d} / P$ DEPENDENCE OF ELECTRON DRIFT VELOCITY FOR DIFFERENT GAS MIXTURES IN A KINESTATIC CHARGE DETECTOR Sreenivas Devidas, George C Giakos and F.A. DiBianca} Department of Biomedical Engineering, University of Tennessee, Memphis, TN 38163

The kinestatic charge detector in brief, comprises a scanning drift chamber whose velocity is synchronized to the drift velocity of the ions produced therein, so as to cause the moving (kinetic) ions to appear to be at rest (static) in the patient rest frame. The X-ray interactions in the gas produce positive ions and electrons. The electrons move towards the high voltage plate which is held at a high positive potential. The positive ions formed move towards the grid which is held at lower potential. The electrons may attach during this transit to other neutral electronegative impurity atoms and form negative ions. The negative ions so formed may recombine with the positive ions and this leads to a signal loss and a consequent reduction in detective quantum efficiency (DQE) because the pulse height distribution is broadened. The addition of a polyatomic gas such as carbon dioxide, methane, hydrogen or nitrogen has been found to reduce the electron attachment to a considerable extent via the Ramsauer Effect. The consequent reduction in the signal loss due to recombination results in a considerable improvement in the signal obtained from the KCD. This improvement depends on the ratio of the drift field to the pressure (Nolts $/ \mathrm{cm} / \mathrm{mmHg}$ ) and the results of this dependence for different gas mixtures will be presented.

320

KCD VS FILM-SCREEN RADIOGRAPHY: A COMPARISON OF TECHNIQUES AND IMAGES

F. A. DiBianca, S. Kollipara and S. Nagarajan.

Department of Biomedical Engineering

University of Tennessee, Memphis 38163

An advanced small-field-of-view Kinestatic Charge Detector (KCD) for digital radiography has been developed and evaluated, and a large-field KCD imager is nearing completion. The new detector uses 80-100 atil krypton gas doped with anmonia and carbon dioxide. Krypton gives higher resolution and less indetector scatter than xenon, ammonia improves resolution in the drift direction by eliminating mobility dispersion, and carbon dioxide reduces electron attachment and the ensuing ion-ion recombination. Initial results indicate that lead bar pattern modulation is visible in the KCD images at $71 \mathrm{p} / \mathrm{mm}$ for moderately high $\mathrm{kV}_{\mathrm{p}}$ techniques, and $\mathrm{x}$-ray quantum detection efficiencies of 80-90 $₹$ have been obtained. In addition, KCD signal output noise corresponding to approximately $4 \mathrm{x}$-ray photons has been observed, mostly due to electronic noise.

The basic imaging techniques for the KCD and film-screen detectors will be compared, as well as the relative spatial and contrast resolution capabilities of the two techniques.

321

UTIUZING ANALOG INTEGRATORS IN LOW LEVEL DIGITAL IMAGING SYSTEMS J.S. LAUGHTER

DEPARTMENT OF BIOMEDICAL ENGINEERING,

THE UNIVERSITY OF TENNESSEE, MEMPHIS

The optimization of electronic image pixel information requires compromises and tradeofis in low signal environments. This paper describes an electronic "front end" for X-ray scanning, consisting of Burr Brown 2101 analog integrator chips, used to collect nanoampre signals over 200 microsecond time periods for subsequent analog to digital conversion and image processing.

The sources of error, analog and digital noise, and control components are discussed and compared to conventional analog electrometer circuitry. Design details such as input guarding and stray input capacitance and these effects on noise and stability are also examined. Design performance is compared to actua! performance of a recently constructed 384 channel system and inferences made to the expectations of a 2304 channel imaging system currently under development.
322

COMPUTER SIMULATION AND EXPERIMENTAL STUDY OF X-RAY SCATTER IN A KCD SYSTEM

Qian Peng, F. A. DiBianca

Department of Biomedical Engineering, University of Tennessee, Memphis, TN 38163

$X$-ray scatter has long been an interesting subject in the development of a KCD digital radiographic imaging system. In this work, we studied the scatter to primary ratio of the KCD for different detection gas under different pressures and combination ratio for a set of different window material and thickness, by using Monte Carlo code and other computer simulation method. We also measured the line spread function and scatter to primary ratio of a KCD, and compared it with the theory. The result may lead to a better understanding of the scatter problem and the optimization of the design for future KCD's. The patient-related X-ray scatter will also be discussed.

323

GRID OPTIMIZATION IN THE KCD

A.Mahmud,F.A.DiBianca,J.Laughter University of Tennessee, Memphis

The Kinestatic Charge Detector is an imaging device that scans the patient by moving the source and the detector across him at a kinestatic speed. The $X$-rays entering the gas detector result in ion formation. The positive ions contribute to the signal generated at the collector. A grid is used to shield the collector, and thus prevent blurring the image. In this paper, an account of the efforts at optimizing the grid is given, so that the noise created by grid microphonics is at a minimum, and also shifted to a higher frequency zone than that at which the detector is normally subject to.

324

$$
\begin{aligned}
& \text { NORMALIZATION OF KCD DATA } \\
& \text { Senthilkumar N., and Dr DiBianca F. A. } \\
& \text { Department of Biomedical Engineering, } \\
& \text { University of Tennessee, Memphis. }
\end{aligned}
$$

The KCD test detector data is distorted due to beam modulation effects, $\mathrm{x}$-ray photon noise, anode surface imperfections, random noise in the detector electronics, etc., This paper discusses the ways to reduce the distortion by normalising the data using a reference signal. Two possible ways of normalizing the data using the KCD reference electrode and CsI beam monitor are explained. An algorithrn to correct CsI detector data before it could be applied to normalize the KCD data has been explained.This correction is needed because CsI detector and KCD are integrating in different time domains. Experimental results are presented. The normalization using the $\mathrm{KCD}$ reference was found to be better than the CsI detector reference. The reasons for the low noise reduction of the CsI reference method are explained. 
325

Off-Resonance Spin Locking for MR Imaging of the Breast

Giles Santyr, Jeff Fairbanks, Fred Kelcz, Jim Sorenson. University of Wisconsin, Madison, WI 53716.

Conventional MR images of the breast often are confusing because of tissue heterogeneity and ambiguous contrast arising from the overlap in relaxation times between lesions and normal breast tissues. Fat suppression is helpful; however, the lack of contrast between fibro-glandular breast tissue and lesions may still be a problem. This is particularly important for younger women with more glandular breasts on whom $X$-ray mamography often is inconclusive. Administration of exogenous contrast media (e.g. GdDTPA) is useful, but is invasive, costly and not suitable for all patients. The purpose of this work was to develop an optimized MR imaging technique for improved detection of breast lesions without the need for contrast media. The technique combines inversion recovery and off-resonance spin locking to suppress signal from both fat and fibro-glandular tissue simultaneously. An anthropomorphic breast imaging phantom was used to optimize the technique for clinical application using a GE Signa MR imaging system (GE Med. Systems, Milwaukee WI.) Preliminary clinical results indicate that the method can provide improved delineation of breast lesions, particularly in women with more glandular breasts.

FLUORESCENCE AND PHOSPHORESCENCE OF BREAST TISSUE AT LOW TEMPERATURE

\author{
G. C. Tang, Pradhan Asima, and R. R. Allano \\ Mediphotonics Laboratory \\ Institute for Ultrafast Spectroscopy and Lasers
}

The City College of New York, NY 10031

The low temperature fluorescence spectra of cancerous and benign tissues excited by $300 \mathrm{~nm}$ were compared with room temperature using CD-Scan. At room temperature, the main peak is at 340 associated with tryptophan and a weak peak around $460 \mathrm{~nm}$ associated with NADH. At low temperature near $77 \mathrm{~K}$, the spectra were wider than that at room temperature and shifted to longer wavelength due to the appearance of the peak at $380 \mathrm{~nm}$ associated with collagen and the partial overlap of $380 \mathrm{~nm}$ band with the $340 \mathrm{~nm}$ band.

At low temperature, the main fluorescence peak of cancerous breast tissue excited by $340 \mathrm{~nm}$ was located at $374 \mathrm{~nm}$ which was shifted by about $5 \mathrm{~nm}$ to shorte wavelength from $381 \mathrm{~nm}$ at room temperature, and the second peak located at $461 \mathrm{~nm}$ was also shifted to the shorter wavelength of $5 \mathrm{~nm}$. A small spectral shift was observed for benign tissue at low temperature.

Phosphorescence band of cancerous tissue excited by $340 \mathrm{~nm}$ was found to be wide in the region from $435 \mathrm{~nm}$ to $540 \mathrm{~nm}$. Phosphorescence band of benign tissue is wider from $435 \mathrm{~nm}$ to $575 \mathrm{~nm}$. The average phosphorescence decay time of cancerous tissue measured at $120 \mathrm{~K}$ was $360 \mathrm{~ms}$ which appeared to be faster than that of $527 \mathrm{~ms}$ of benign tissue.

This work is supported by Mediscience Technology Corporation.
327

QUANTITATIVE ANALYSIS OF PHANTOM IMAGES IN MAMMOGRAPHY

Dev P. Chakraborty Ph.D. and Gerald R. Edeistein M.S.

Department of Radiology, Hospital of the University of

Pennsylvania, 3400 Spruce St, Philadelphia, PA 19104

The American College of Radiology (ACR) accreditation program for mammography includes subjective phantom image quality evaluation as part of the accreditation process. The ACR phantom contains several target structures (fibrils, microcalcifications and masses) whose visibilities are rated by a group of medical physicist readers. Performance on this imaging test is a significant factor in the decision to pass or fail a facility. This study was aimed at determining if improvements are possible 10 this $\mathrm{men}$ are possible 10 this methodology if one employs quanithative measurements on the phantom images instead. Several images of the AC different imaging conditions ( $\mathrm{kVp}$ and grid) were digitized at high spatial and gray scale resolutions ( 50 micron pixels and 12 bits per pixel) on a laser digitizer (LUMISYS LUMISCAN 100). Images were analyzed using a high resolution display station (2 DOME Imaging Systems MD Series boards with $20 \mathrm{MB}$ image memories and $1200 \mathrm{X}$ $1600 \times 8$ bit display memories, 2 Image Analysis Monitors, and a Intel 486 based personal computer). The analysis consisted of computing suitably normalized pumerical indices comesponding to each of the target structures in the phantom. numerical indices corresponding to each of the target structures in the phan. Companisons were images and the numerical indices. The initial results suggest that the numerical analysis is more sensitive at detecting subtle image quality variations in the phantom images.

This work was supported in part by a grant from NIH (1RO1-CA54788)

328

SCREEN - LENS - CCD FOR MAMMOGRAPHY

H.D. Zeman

Dept. of Biomedical Engineering, University of Tennessee, Memphis

An experimental imaging system is being designed which will apply the high quantum efficiency and high spatial resolution possible with a transparent crystal intensifying screen combined with the high accuracy in light measurement possible with cooled CCD technology towards the improvement of mammographic detection of cancer in a fashion that ultimately will lead to earlier diagnosis and better differentiation of benign from malignant disease. The scintillating crystals under study include CsI(TI), a GE proprietary ceramic made of $\mathrm{Gd}_{2} \mathrm{O}_{3}(\mathrm{Eu})$, and the glassy scintillating phosphor coatings used in military CRT displays. The lens designs being considered have a speed of at least $f 0.65$ and a magnification of 1:1. The design of a $2 X$ magnitying lens which when used with a $80 \mathrm{~mm}$ f 1.3 (2:1 demagnitying) oscilloscope camera lens will achieve f 0.65 speed and 1:1 magnification has already been completed. The light detector for the system will be a Tektronix $2048 \times 2048$ pixel thinned CCD, thermoelectrically cooled to at least $-80^{\circ} \mathrm{C}$. The imaging systern will have a field of view of $49 \times 49 \mathrm{~mm}$ and a spatial resolution of 24 $\mu \mathrm{m}$. This system is being designed in collaboration with Science Applications International Corp., and GE Research Laboratories. 
329

VOLUMETRIC 3-D ULTRASOUND IMAGING

STEVE CHARLES, M.D.

CENTER FOR RETINA VITREOUS SURGERY

Cl and specific values for each voxel. Because reflection ultrasound is dependent on speckle or reflection, it is impossible to assign a specific value to a pixel in 2-D imaging or voxel in 3-D imaging. All CRTs are intrinsically planar displays (2-dimensional). The use of liquid crystal light valve technology for field sequential stereo permits a pair of planar projections of a volume but is not a true volumetric display. Motion parallax is the most powerful cue for the human observer to interactively decode volumetric information from an intrinsically 2-dimensional planar CRT display. Utilizing a volumetric ultrasound method developed by Drs. Rob Entrekin and Philip Keller of ATL, referred to as Slit-o-Vision, it is possible to acquire volumetric reflection ultrasound information. This then is decoded by the human observer moving the acquiring probe while visualizing the monitor principally based on motion parallax. 
331

MASS SPECTROMETER PROBE MEASURES AQUEOUS PHASE $\mathrm{O}_{2}, \mathrm{CO}_{2}$ AND INERT GAS TENSIONS WITH MINIMAL UNSTIRRED LAYER

JE Baumgardner, JA Quinn, GR Neufeld

Depts Anesthesia, Bioengineering, and Chemical Engineering

University of Pennsylvania, Philadelphia, PA

Membrane inlet systems for mass spectrometers have shown great utility in the quantitative analysis of nonpolar, low molecular weight compounds in aqueous solutions. The dimensions required for a membrane of suitable area have restricted their miniaturization and their application to measurements of gas tension gradients in in-vitro and in-vivo models of the microcirculation. We have developed a membrane inlet system for a mass spectrometer that excludes water and polar compounds and admits gases into the mass spectrometer for analysis. We have used this system to measure gas tensions of oxygen, carbon dioxide, helium, argon, and nitrous oxide in aqueous solutions prepared for calibration of the probe. The system is highly linear. The signal to noise ratio for oxygen at $0.5 \mathrm{~atm}$ is 8.4 and is increased to 26 by repeated measurements over 30 minutes. The probe is physically large, at the tip of 16 Ga tubing, but the sampling pore is very small, estimated to be 40 angstroms. The sample rate for $100 \%$ oxygen is $2 \times 10^{*}-13 \mathrm{ml} \mathrm{O} 2 / \mathrm{min}$; at this low sample rate, there is no defectable stirring effect and the depletion layer in the liquid phase around the sample pore is estimated to be less than one micron. The combination of a small sampling pore and a very low sample rate should allow adequate spatial resolution to measure gas tension gradients on a microcirculatory scale.

332

CHARACTERIZATION OF BIOSENSOR RESPONSE WITH RAMP PERTURBATIONS Dale A. Baker and David A. Gough

Department of Applied Mechanics and Engineering Sciences, University of Califomia, San Diego La Jolla, CA 92093

Traditionally, sensor transient response has been characterized by the response to a step concentration change. Response time is reported as the time required for the sensor to reach a specified percentage of the final steady state response, for exampie, the " $95 \%$ response time." This approach is usually satisfactory for biosensors operated in a discrete sample mode, where a sample is taken from the biological environment and briefly exposed to the sensor. However, for biosensors that are intended for direct contact with the biological environment and operate continuously, such as implantable biosensors, use of a step "response time" or "time to reach steady state" is of limited value in characterizing the dynamic response to changes seen in situ.

We propose that a useful characterization for biosensors, which are used for continuous monitoring applications, can be performed with ramp concentration changes. This perturbation is monitoring applications, can be performed with ramp concentration changes. This perturbation is
relevant for testing and analyzing the response of continuously operated biosensors since actual relevant for testing and analyzing the response of continuously operated biosensors since actual
monitoring conditions are better approximated by ramps than by abrupt step changes. The ramp rate can be adjusted to simulate the analyte's expected rate of change.

The response of an enzyme electrode for glucose will be presented as an example of a continuously operated biosensor that has been characterized with ramp concentration perurbations. It will be shown that for a ramp perturbation at a fixed rate, there is an initial lag in the response of the sensor after which the response follows the ramp input with the same slope. The dynamic delay is the parameter that indicates the extent to which this response is simply shifted in time. There is also a dynanic error, which is the the difference in concentration indicated by the sensor and the actual concentration. The dynamic delay and dynamic error increase as the ramp rate increases. It will be shown that the maximal rate of change of the analyte can be used to give an upper bound on the dynamic delay and dynamic error.

333

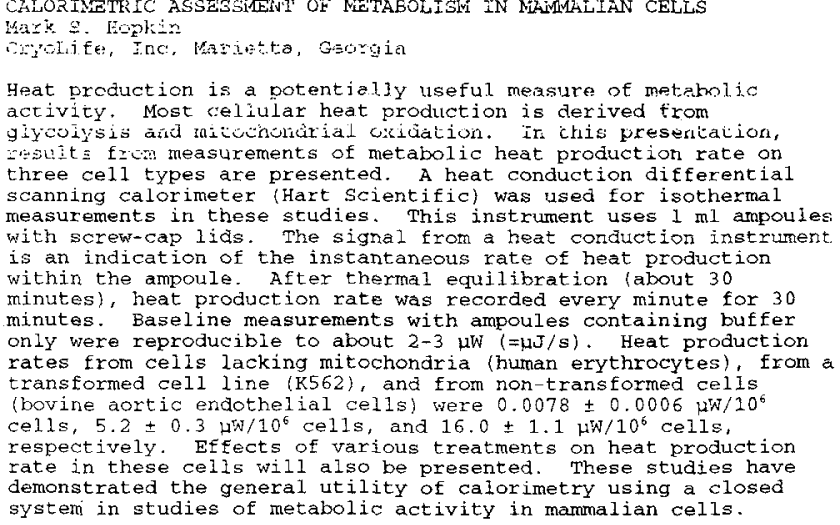

334

Design and Evaluation of a Novel In Vivo Ultrasonic Wall Shear Rate Measuring Device. Keynton, R.S., Neifert, Q.Y., Nemer, R.E., Fatemi, R.S., and Rittgers, S.E. Department of Biomedical Engineering, The University of Akron, Akron, OH

Hemodynamics, especially low wall shear stress, play a key role in the development of atherosclerosis and bypass graft intimal hyperplasia. Wall shear stress has often been obtained in vivo from either estimates based on volume flow rate [Binns et al., JVS, 89] or single-point velocity measurements, in conjunction with the wall no slip assumption and a simple linear regression. However, previous experiments in our lab [Fatemi et al., ASME, Summer '93] have shown that, under pulsatile flow conditions, wall shear rates are more accurately predicted (within $\pm 20 \%$ of theoretical values) by using 4 velocity points ( 3 free stream points together with the no slip assumption at the wall) and a $3^{\text {rt }}$ order polynomial curve fit. Therefore, it is the purpose of this paper to evaluate the accuracy of a new, in vivo transducer capable of determining wall shear rates non-intrusively from velocities at three points along a line perpendicular to the vessel wall.

Three $20 \mathrm{MHz}$ ultrasound crystals $(\mathrm{d}=1 \mathrm{~mm})$ were imbedded in an elastomer at spacing of 1.5 and $2.1 \mathrm{~mm}$ with beam angles of $30^{\circ}, 45^{\circ}$, and $60^{\circ}$ to the horizontal plane. Optical examination with a viden light microscope showed that inter-crystal spacings were within $3.9 \pm 5.1 \%$ and crystal angles were within $2.6 \pm 3.5^{\circ}$ of the design. Calibration was performed under steady $\left(\mathrm{Re}=258\right.$ ) and pulsatile (lliac artery: $\alpha=3.5, \mathrm{Re}_{\mathrm{peat}}=1000, \mathrm{Re}_{\mathrm{man}}=200$ ) flow conditions. Mean point velocity measurements were within $-3.4 \pm 6.7 \%$ and $5.8 \pm 8.7 \%$ compared to theoretically predicted steady and pulsatite values, respectively. Furthermore, peak systole point velocity measurements were within $2.4 \pm 6.2 \%$ and oscillatory velocities were within $2.7 \pm 7.6 \%$ accuracy. Steady and pulsatile average shear rates were calculated and found to be $26.5 \pm 4.9 \mathrm{~s}^{-1}$ and $49.0 \pm 8.4 \mathrm{~s}^{-1}$ compared to theoretical values of $28.1 \pm 0.2 \mathrm{~s}^{-1}$ and $47.3 \pm 2.2 \mathrm{~s}^{-1}$, respectively. In a preliminary in vivo investigation, the average and peak systolic shear rates in a dog carotid artery were found to be $433.4 \mathrm{~s}^{-1}$ and $1144.5 \mathrm{~s}^{-1}$, respectively, which correlate well with values obtained by others [Binns et al., JVS, '89]. Results from this study show this device to be a viable technique for measuring wall shear rates in vivo.

335

BLOCKING OF EDRF IN ISOLATED RAT CEREBRAL ARTERIOLES: MEASUREMENT OF INTRACELLULAR CALCIUM AND VESSEL DIAMETER Hans H. Dietrich and Ralph G. Dacey, Jr. Dept. of Neurosurgery, Washington University, St. Louis, MO, 63110

Penetrating arterioles $(n=8)$ of rat cerebral cortex were isolated and cannulated according to Dacey et al. (Am.J.Physiol. 243:H598-H606; 1982). Arteriolar smooth muscle cells were loaded with the calcium sensitive probe FURA-2 $(5 \mu \mathrm{M})$ and subjected to various extraluminal stimuli. Using a ratiometric fluorescence video imaging system, the intracellular calcium concentrations $\left(\mathrm{Ca}_{\mathrm{i}}^{*+}\right)$ in the smooth muscle cells were measured as indicated by the ratio of two observed wavelengths $(340 \mathrm{~nm} / 380 \mathrm{~nm})$. $\mathrm{Ca}_{\mathrm{i}}{ }^{*+}$ was correlated to the simultaneously measured intraluminal vessel diameter measured with a video-dimensional system. All vessels developed

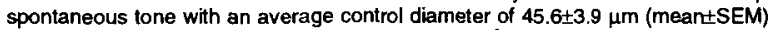
and $\mathrm{Ca}_{i}{ }^{++}$of $111 \pm 13 \mathrm{nM}$ at $\mathrm{pH} 7.3$. Papaverine $\left(10^{-5} \mathrm{M}\right)$ and acidic $\mathrm{pH} 6.8$ dilated the vessels to $61.0 \pm 6.6 \mu \mathrm{m}$ and $57.8 \pm 5.8 \mu \mathrm{m}$ as well decreased the $\mathrm{Ca}_{1}^{*+}$ to $55.6 \mathrm{nM}$ and $78.3 \mathrm{nM}$, respectively. Alkaline $\mathrm{pH}$ of 7.65 and the calcium ionophor ionomycin $\left(5^{*} 10^{-5} \mathrm{M}\right)$ constricted the arterioles $1034.8 \pm 3.9 \mu \mathrm{m}$ and $30.0 \pm 5.8 \mu \mathrm{m}$ with an increase in $\mathrm{Ca}_{1}^{++}$to $145 \pm 3.9 \mathrm{nM}$ and $825 \pm 652 \mathrm{nM}$, respectively. These results show that there is an inverse proportional relationship between vessel diameler and $\mathrm{Ca}_{+}{ }^{*}$. Surprisingly, blocking the production of endothelium derived relaxation factor (EDRF) with $\mathrm{N}$-omega-nitro-L-arginine (NOLA, $10^{-5} \mathrm{M}$ ) constricted the vessel to $34.4 \mu \mathrm{m}$ without raising but lowering $\mathrm{Ca}_{i}^{+*}$ to $77.0 \mathrm{nM}$. The dissociation of vessel tone and $\mathrm{Ca}_{i}^{++}$after blocking the EDRF production with NOLA has not been reported before in cerebral arterioles. This observation may indicate that EDRF regulates vascular tone in a calcium independent way such as $\mathrm{Ca}_{i}^{+*}$-independent cGMP-cyclase (Khalil,R.A. et al., Am.J.Physiol. 263:C714-C718, 1992). Supp. NIH/NHLBI 5 RO HL37983-05.

336

ANAEROBIC THRESHOLD AND MAXIMUM OXYGEN CONSUMPTION WITH RESPIRATOR MASKS

A. T. Johnson and C. R. Dooly

Biological Engineering

University of Maryland

College Park, MD 20742

Construction of a comprehensive mathematical model to predict exercise tolerance of people wearing respirator masks requires knowledge about the physiological effects of mask wear. It is known, for instance, that mask resistance induces hypoventilation but mask dead volume causes hyperventilation. Several consequences of mask wear are not known: do masks influence lactate and ventilation thresholds during exercise, and do masks cause changes in maximum oxygen consumption?

Ten trained bicyclists were tested with and without masks on a bicycle ergometer. A stepwise exercise protocol was used with 3-minute work rate plateaus. Blood drawn from the antecubital vein during the last minute of each plateau was analyzed for lactate. Ratios of minute ventilation to oxygen consumption were used to determine ventilation thresholds. Maximum oxygen consumptions were also determined. Results will be used to help define the comprehensive model mentioned earlier. 
337

ANTERIOR CRUCIATE LIGAMENT (ACL) IMPLANT TENSION MEASUREMENT William D. McLeod, Ph.D.

Smith \& Nephew Richards Inc., Memphis, Tennessee

In the event of a disruption of the $A C L$, it is generaliy agreed upon that attempts to repair this structure have shown to be ineffective forcing the need for a replacement. The strain environment of the replacement device is dependent not only upon the location of the implant relative to the femur and the tibia, but upon the tension of the implanted device. This study compares the influence of the stifness of the tensiometer on the actual strain imposed on the ligament replacement device. There is a significant difference between the load actually applied to the implanted device by the femoral-tibial articulation than was predicted where the spring constant of the implanted device differed substantially from the spring constant of the tensiometer. The influence of the position of the implant device on the strain environment was investigated. From these measurements it is shown that it is possible to approximate the normal joint laxity using a prosthesis and carefully controlling the implant tension.

338

THE MECHANICAL EFFECTS OF THE SUPERIOR CHECK DESMOTOMY ON THE SUPERFICLAL DIGITAL FLEXOR TENDON

HA Lansari, CF Abrams Jr, KF Bowman, DG Bristol

Department of Biological and Agricultural Engineering

Department of Food Animal and Equine Medicine (Bowman, Bristol)

North Carolina State University

The purpose of this study was to evaluate and model the mechanical effects of superior check desmotomy on the superficial digital flexor(SDF) system. Fresh horse limbs were dissected out leaving the SDF muscle, tendon, and proximal check ligament (SDF system) intact. Force-elongation characteristics of the SDF system were measured using Instron Materials Testing System. The distribution of elongation along the tendon and muscle was measured using a contact-type strain gage extensometer. Characteristics were measured before and after superior check desmotomy. For the same overall elongation, tendon strain decreased while muscle strain increased as a result of check desmotony. The force-elongation characteristics of the SDF muscle and tendon were determined from the Instron tests and used to develop a non-linear, discrete parameter mathernatical model of the SDF system to simulate the effects of the superior check desmotomy. The model was developed using hardening springs and was found to adequately describe the mechanical response of the system. The utility of the model in evaluating the effects of varying tendon, muscle, and check ligament elastic parameters, simulating injury or surgical interventions, is demonstrated.
339

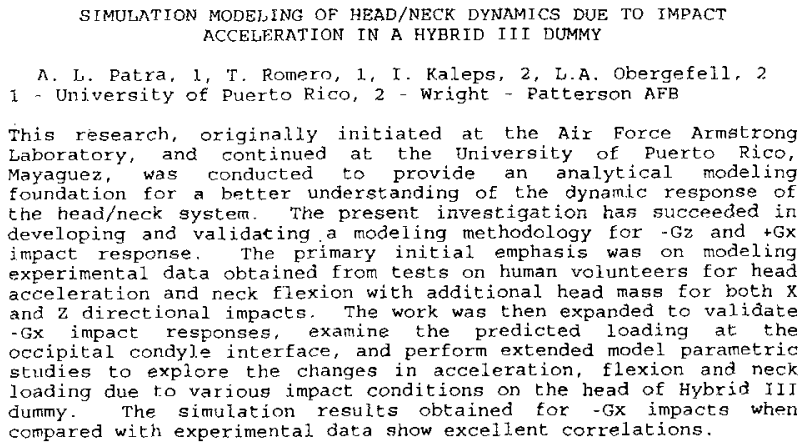

340

UNSOLVED PROBLEMS IN ORTHOPAEDIC BIOMECHANICS

James H. Calandruccio, M.D.; Claiborne A. Christian, M.D.; Stanley C. Graves, M.D.; James L. Guyton, M.D.; William C. Waner, M.D.; and Keith D. Williams, M.D.; Campbell Clinic, Memphis, TN

In this session, practicing orthopaedic surgeons will each present an unsolved problem in each of the following subspecialties: hand surgery, sports medicine, foot and ankle, total joint arthroplasty, pediatrics and spine. The orthopaedic surgeons will concentrate on the clinical importance of the problem as well as the apparent limitations which have prevented a solution. The floor will then be opened for discussion between the biomechanical engineers in the audience and the orthopaedic surgeons creating dialogue between the professionals of both disciplines. The hope is that new light may be shed on potential solutions to the problem presented, and possibly, the development of meaningful collaborative research. 
341

THE MULTI-DISCIPLINARY ROLES OF BIOENGINEERS IN CUSTOM ORTHOPAEDIC DEVICE MANUFACTURE

Caroline W. Wang

Johnson \& Johnson Orthopaedics, Inc., Raynham, MA

In developing custom orthopaedic devices, the role of the biomechanical engineer is multifaceted and is dependent on many skill sets. The engineer optimizes the performance of the desired device, first taking into consideration anatomical features, surgical technique, and patient activity level. These requirements are extracted from $x$-rays, CT's, communication with the surgeon and often the surgeon's sales representative, as well. Understanding these needs require a knowledge of normal and abnormal bone physiology, and different surgical approaches and philosophies. Second, the engineer confirms that the component that $\mathrm{s} / \mathrm{he}$ designs will perform under the rigorous conditions of the patient's daily life. This evaluation includes material selection and may involve a simple calculation or a complex FEM simulation. Proficiency in computer analysis and $\mathrm{CAD}$ allows the engineer to modify the design easily. Within these parameters, the engineer assesses manufacturability, cost, and the lead time required to produce the device. Interaction with machine operators, inspectors and packaging personnel is crucial to the success and timeliness of the delivery of the implant. In most cases the need for a custom component is urgent, and all of these factors must be weighed carefully. In custom orthopacdic device manufacture, the engineer relies on clinical knowledge, engineering expertise and a professional demeanor to meet the needs of the patients, surgeons and hospitals in an efficacious, timely and cost-effective manner.

342

\section{DIATHRODIAL JONT MECHANICS}

Jennifer S. Wayne

Divisions of Orthopaedic Surgery and Biomedical Engineering

Medical College of Virginia, Virginia Commonwealth University

Diarthrodial, or synovial, joints are the freely moving articulations that provide a large degree of motion between opposing bones, such as in the shoulder and knee. They are subjected to widely varying conditions of loading yet are capable of functioning effectively for many decades. Although the mechanics are complex and involve numerous facets, two critical issues must be appreciated to understand the mechanical ability of the diarthrodial joint: the lubrication mechanisms present and the behavior of the articular cartilage. Several lubrication theories (i.e. elastohydrodynamic, squeeze-film, weeping, and bocsted lubrication) have been postulated to cxist in the joints under different circumstances. These mechanisms provide a film of synovial fluid between the articulating bones which is pressurized and transmits the stresses on the joint the cartilage. Because articular cartilage functions as a two phase biological material (i.e. consisting of a solid and fluid phase), the extent to which the applied stress to the cartilage is supported by the two phases greatly influences the mechanical behavior, and thus the homeostasis, of the tissue.

343

ROLE AND FUTURE OF FINITE ELEMENT MODELING IN DIARTHRODIAL JOINT BIOMECHANICS

Jun-Kyo Suh

Department of Orthopaedic Surgery, University of Pitusburgh

The relationship between structure and mechanical function of diarthrodial joint is very important for understanding the mechanisms behind the osteoarthritic change of joint cartilage and its surgical intervention. However, the geometric complexity of the joint along with the macro and micro-structural complexity of its components conscitute an exuremely intricate mathematical problem. For example, the cartilage is a multi-component biological tissue, consisting of solid extracellular structural matrix (collagen, proteoglycan and other minor proteins) and interstitial mobile fluid, whose interactions play an important role for the viscoelastic characteristics of the whole tissue. The set of the multi-phasic momentum equations for the physiological configuration can only be solved through numerical methods. Recently, several different kinds of finite element modeling of this problem have been developed along with appropriate interface boundary condirions between the joint synovial fluid and the cartilage tissue. The development of these numerical simulation techniques open new avenues toward further exploration of the 3-D physiological computer simulation of diarthrodial joint biomechanics.

344

HISTOLOGIC AND BIOMECHANICAL COMPARISON OFF INGROWTH SURFACES IN DYNAMICALIY LOADED AND UNLOADED ANIMAL MODELS Harold M. Aberman

Howmedica Division of Pfizer HPG Inc., Rutherford, NJ

Over a decade of experience has shown cementless porous coated implants to have variable suocess in osseointegration of the bone-implant surface. New ingrowth surfaces, biomaterials and surface coatings are being investigated to determine if the interface can be optimized regardless of the patient history and metabolic bone status. Retrieval PCA hip specimen were compared to anatomic, cementless, porous coated canine femoral components to determine the validity of the canine model. Following successful comparison of bone ingrowth and surface strain changes between the human and the canine femur, a series of studies were conducted to look at bony reaction to madreporique surfaces, hydroxyapatite coated ingrowth surfaces, cast mesh surfaces, diffusion bonded titanium mesh surfaces, and composite ingrowth surfaces. For these various porous surfaces, an anatomic canine femoral component was used as part of a total hip replacement and/or a transcondylar femoral plug study was done to determine bony reaction to the ingrowth surface. Similarly, a study investigating the effect of age and hormonal status was conducted to determine the effectiveness of hydroxyaparite coatings in young, old, and osteoporotic bone.

345

EFFECT OF MICROMOTION OF INGROWTH INTO POROUS COATED IMPLANTS J. Marcus Hollis, Charles L. Stewart, Otto E. Hofmann and Charkene M. Flahiff Department of Orthopaedic Surgery - University of Arkansas for Medical Sciences

Porous coated implants are gaining acceptance for total joint replacement. However, clinical results to date indicate that bone ingrowth into porous coatings is often far from complete. Several factors such as pore size, coating thickness, percent porosity, interface gap and interfacial micromotion are thought to affect the extent of bone ingrowth into porous coated implants. The purpose of this study was to investigate the effect of micromotion on porous ingrowth into implants in cortical bone. The animal model consisted of four cylindrical porous-coated titanium plugs which were implanted transoortically into lateral canime femurs. Implants were placed in the contralateral side as controls. Each day post surgery the four experimental implants were moved $25,50,100$, or 200 microns respectively in rotation for 10 minutes twice a day. The dogs were sacrificad and shices of the implant-bone ingrowth was calculated. Implants from the control side that had no applied motion showed the least amount of ingrowth with an average of less than 50 percent. Motion groups with 25-100 microns of motion all displayed from 85 to 90 percent of ingrowth. Implants with the 200 microns of motion had significantly reduced ingrowth when companed to the other groups. It appears from these results that there is a range of motion that is optimal for bony ingrowth. One possible explanation is that a limited amount of micromotion will actually induce bone formation into the pores. It was also shown that just 10 minutes of motion twice a day was enough to inhibit ingrowth into the specimens with 200 microns of motion. This could be due to disruption of ingrowth that could not recover before the next disruption by the application of motion.
346

BIOMECHANICAL ANALYSIS OF PATEILAR TENDON ALLOGRAFTS Charlene M. Flahiff, Andrew T Brooks and Richand W. Nicholas Department of Orthopaedic Surgery - University of Arkansas for Medical Sciences

Patellar tendon allograft is the most frequently used allograft for anterior cruciate ligament (ACL) reconstruction. The lower morbidity, decreased operating time, and quicker recovery associated with allografts, as opposed to antografts, have lead to increases in their usage. While patellar tendons are usually obtained from donors less than $\mathbf{4 0}$ years of age, no specific donor age limits have been established, primarily due to lack of mechanical data. The purpose of this study was to evaluate the biomechanical properties of patellar tendon allografts from donors 20-55 years of age. Bone-patellar tendon-bone specimens were harvested from acceptable donors, processed, and prepared as for surgery. Soft tissues were removed, and the tendon was sectioned lengthwise to the central thind. Areas and length measurements were taken. The patella and tibial tubercle remained intact and were pottod in low melting point metal. Specimens were pulled to failure using an MTS test system at a rate of $10 \%$ of the initial length per second. Force at failure, ultimate tensile stress, percent elongation, and modulus of elasticity were determined for each specimen. There was no significant difference ( $p>0.05$ ) for any of the mechanical properties with respect to age. Load at failure ranged from $2110 \mathrm{~N}$ to $4650 \mathrm{~N}$ with a mean of $3440 \mathrm{~N}$. Regression analysis showed decreasing tensile strength with increasing age, but the correlation was not significant. It appears that patellar tendon allografts from donors up to age 55 may be of sufficient strength for ACL replacement. 
347

CLINICAL AND EXPERIMENTAL ASPECT OF ORTHOPAEDIC BIOMECHANICS Thay Q Lee, Guisou Mahmoud, William C. Kim and Sanford H. Anzel Department of Orthopaedic Surgery, University of California, Irvine and Long Beach VAMC

Human joints are subjected to a wide range of forces during the activities of daily living. With athletic activities, diarthrodial joint forces can increase to as high as 10 to 20 times body weight. In case of diseased or injured joints, the structure and function must be restored using biological or prosthetic components. Resurfacing or reconstruction of the knee, hip and the shoulder are part of the daily routine for orthopactic surgeons and it is this clinical basis that necessitates a strong need for basic science knowledge of both biology and mechanics (i.e. orthopaedic biomechanics). For example, in total knee arthroplasty (TKA), patellofemoral complications arise from inadequate restoration of the patellofemoral structure, kinematics and also from excessive contact pressures observed in the resurfaced patellofemoral joint. A biomechanical study in our laboratory using fresh human cadaver knees showed that the patellofemoral contact pressures increased up to six times after TKA when compared to normal patellofemoral contact pressures. In addition, the patellofemoral contact area decreased to approximately $10 \%$ of the normal patellofemoral contact area for kmee flexion angles ranging from $30^{\circ}$ to $120^{\circ}$. For this reason, after TKA, a greater tendency toward excessive wear and loosening of the misaligned patellar prosthesis can be anticipated. In this session, many aspects of engineer's role and perspective in orthopaedic biomechanics research will be explored: fluid lubrication of diarthrodial joints; FEM in biomechanical analysis of articular cartilage; biomechanics of ACL roconstruction; factors influencing cementless fixation of implants; and role of engineers in manufacturing of custom implants.

348

RNOWLBDEB-BASED STRESS MMALYSIS OF CUSTOE BIPS Gregory A. Gray, Robert Paxson, and Ls i Belec, Ph.D. 38002 Introduction -- The wide variation in patient anatomy and the successful press "fit hip replacement, has lead to the use of many

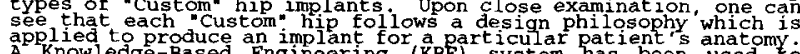
applied to produce an implant for a particular patient s anatomy
Anowledge-Based Engineering (KBE) system has been used to
capture several hip implant design philosophies so that engineering analysis can be performed on each implant. For the knowledge base which can be used for any hip implant design. The
analysise allows the user to define the application point
magnitude, and orientation of the load, and the level for anantede, and orientation of the load and the level for
mant indever fixation. The module can account for manufacturing cantilever fixation. The module can account for manufacturing
tolerances and can calculate axial, bending torsional, shear,
and VonMises stress. The user can evaluate peak VonMises stress

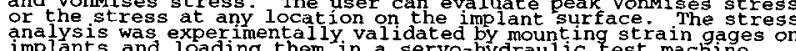

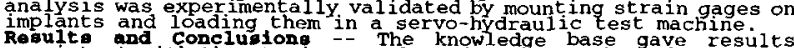

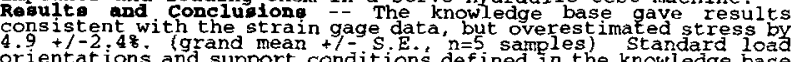
orientations and support conditions def samples st standard load
are used in conjunction with fat ique strengthowledge base are used in conjunction with fatique strength limits and limits for each implant. Two load orientations and corresponding
stress analyses are typicaliy applied to each patient s implant.
Both assume cantilever fixation as recommended by the astM. The Both assume cantilever ixation as recommended by the AsTM, The stresses seen when rising from a chair are simulated in the second analysis. Although these analyses represent "worst case" reserved for commerciali ave engineering analysis formerly

349

COMPUTED TOMOGRAPRY DOSTHETRY OP A CUSTOK HIP IMPLANT PROTOCOL

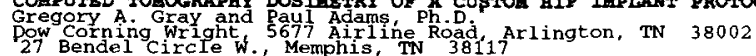

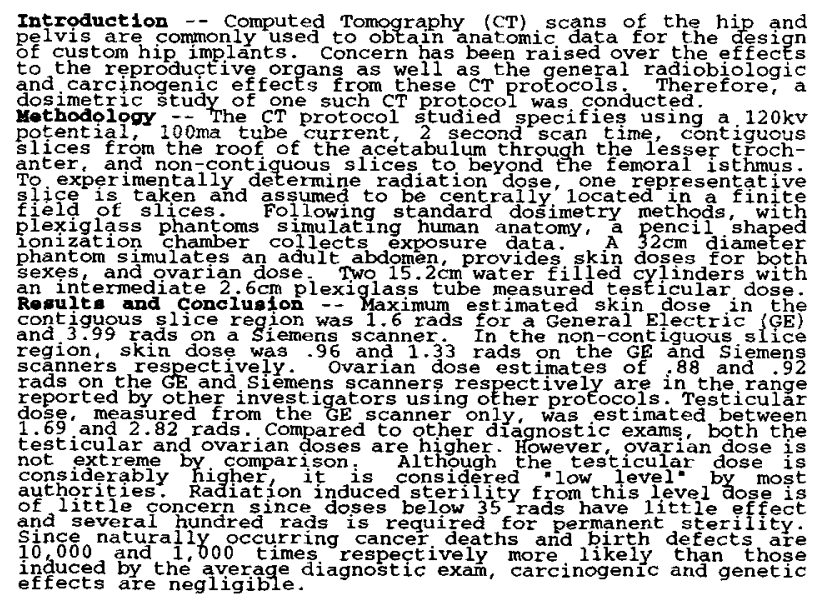

350

QUANTIFICATION OF RELATIVE MOTION BETWEEN AN UHMWPE TIBIAL INSERT, AND METAL TIBIAL IMPLANT

K.P. Daigle, P.E, and D. Melancon, B.S

Smith and Nephew Richards, Memphis, TN

Debris generated from a total joint implant has been associated with adverse biological response. Current research has determined that polyethylene debris in sufficient quantities can initiate this adverse response which may lead to subsequent implant failure. The articulation in a total joint replacement involves the relative motion between a metal femoral bearing surface, a polyethylene patella component and a polyethylene counter bearing tibial surface. This articulation produces UHMWPE debris in a total knee replacement. However, some questions have been raised as to the potential amount of additional polyetheylenc debris generated from relative motion the polyethylene tibial surface and the metal base plate. This study describes a method to quantify the amount of relative motion (and hence, relative wear) between a UHMWPE tibial insert and metal tibial tray as a function of loading cycles.

A Mitutoyo model 513-202 dial gage was used to measure relative motion of different types of tibial implant designs. This gage had a resolution 0.0005 inches (12.5um). The relative motion between the insert and tray was measured at regular intervals to 10 million cycles. The amount of between the insert and tray was measured at regular intervals to 10 million cycles. The amount of 0.005 inches (25.4 $\mu \mathrm{m}$ to $127 \mu \mathrm{m})$ and tended to decrease with increasing load cycles. This study showed that it is possible to assess relative motion in a tibial implant.

351

Changes in Contact Stress Caused by Variations in Poisson's Ratio, Stiffness, and Friction in Plastic Acetabular Liners

M.E. Harbaugh

Smith \& Nephew Richards

This study uses the finite element method to determine the contact stress in the acetabular liner. Nonlinear gap elements were used to represent the femoral head/liner interface. Both conforming and nonconforming femoral geometries were
studied. The effect of liner modulus, Poisson's ratio, and friction on contact stress was studied. The effect of liner modulus, Poisson's ratio, and friction on contact stress was determined. Results are also compared to those predicted from using an analytic method. A $28 \mathrm{~mm}$ femoral component with a $6 \mathrm{~mm}$ thick plastic acetabular liner was modeled. A 3000 Newton $(675 \mathrm{lb})$ load was applied to the femoral head. The outside radius of the liner was considered fixed. UHMW

For a conforming geometry the difference in contact stress between regular and Hylamer PE was less than $1 \%$. The analytic equation underpredicts the contact stress for Poisson ratios less than 0.41 and overpredicts the stress for ratios greater than 0.41. As the coefficient of friction is varied from 0.00 to 0.18 the contact stress decreases by $14 \%$. For nonconforming geometry the contact stress in the Hylamer was $125 \%$ of the contact stress in regular PE. For Poisson ratios greater than 0.40 the analytic equation severely overpredicts the contact stress. As the coefficient of friction is varied from 0.00 to 0.18 the contact stress decreases by $4 \%$ (Figure 1 ).

352

MODELING HEAT TRANSFER IN CORTICAL BONE DURING DIRECT TROCARTIPPED PIN INSERTION

A Lansari, CF Abrams Jr, DJ DeYoung

Department of Biological and Agricultural Enginecring

Department of Companion Animal and Special Species Medicine (DeYoung)

North Carolina State University

Direct power insertion in bone is used in modern orthopedic surgery in cases where, because of cost or other reasons, pre-drilling is not practicable. Many intramedullary pins have a trocar tip which are not only mechanically stronger than other types of surgical pins but also are more economical. However, the pin insertion process may produce excessive heat that causes bone necrosis. A heat transfer model that predicts temperatures in location very close to bone (0.1- $2.5 \mathrm{~mm})$ was derived. It is a diffusion model which includes the mechanical and thermal properties of bone and pin. The heat generation term is derived from specific cutting energy calculations. The model describes the phenomenon of heat diffusion during the insertion process and the simulated temperatures are in good agreement with measured values. The model can be used to predict the temperatures at the pin/bone interface (were the highest temperatures theoretically occur but also were it is very difficult to measure temperature) in future pin tip designs. 
353

ON THE RELATION BETWEEN ENERGY DISSIPATION AND BONE FORMATION IN A MODEL OF SKELETAL ADAPTATION Kenneth J. McLeod, Ted S. Gross, and Clinton T. Rubin Musculo-Skeletal Research Laboratory, Department of Orthopaedics State University of New York, Stony Brook, NY 11794-8181

Using the isolated avian ulna model of disuse osteopenia, we have previously shown that the initiation of new bone formation is a frequency dependent process. For constant daily loading regimen (600 seconds/day) the amount of new bone formed over an eight week period increases monotonically with frequency up through $60 \mathrm{~Hz}$. However, such a loading protocol does not permit a differentiation of the individual effects of changing the number of loading cycles, increasing the strain rate, or changes in loading frequency per se. To continue the investigation of these issues, we have undertaken a study of the interactions between loading frequency, amplitude, and duration in the ability of mechanical loading to prevent the loss of bone mass associated with disuse. The preliminary results demonstrate a distinct relationship between loading amplitude and duration. Specifically, we have shown that with $1 \mathrm{~Hz}$ loading, mass can be maintained with either a $1000 \mu \varepsilon$ peak loading for $100 \mathrm{~s} /$ day or a $600 \mu \varepsilon$ loading for $600 \mathrm{~s} / \mathrm{day}$, while at $30 \mathrm{~Hz}, 300 \mu \varepsilon$ for $600 \mathrm{~s} / \mathrm{day}$ or $150 \mu \varepsilon$ for $3600 \mathrm{~s} /$ day is sufficient to maintain bone mass. These data indicate that, independent of loading frequency, the bone remodeling response will be proportional to loading duration and the square of the peak strain amplitude. The results are consistent with an energy dissipation mechanism of transduction.

\section{4}

A MODEL FOR THE EXCITATION OF OSTEOCYTES BY MECHANICAL LOADING INDUCED BONE FLUID SHEAR STRESSES

S. Weinbaum, S. C. Cowin and Yu Zeng

The City College of The City University of New York

A new experimentally testable hypothesis is advanced for the mechanosensory transduction mechanism by which communicating osteocytes sense the very small in vivo strains in the calcified matrix components of bone. We propose that the osteocytes, although not responsive to substantial fluid pressures, can be stimulated by relatively small fluid shear stresses acting on the membranes of their osteocytic processes. Biot's porous media theory is used to relate the combined axial and bending loads applied to a whole bone to the flow past the osteocytic processes in their canaliculi. In this theory the bone pores of interest are the proteoglycan filled fluid annuli that surround the osteocytic processes in the canaliculi. We show that previously predicted fluid pore surround the osteocytic processes in the canaliculi. We show that previously predicted fiuid pore pressure relaxation times were a hundred fold too short for the lacunar-canalicular porosity because they neglected the fluid drag associated with proteoglycan matrix on the surface membrane of the osteocyte and its cell processes. The recent theory developed in Tsay and Weinbaum (1991) fo flow through cross-linked fiber filled channels is used to model the flow through this proteoglycan matrix and the predicted pore relaxation time, 1-2 sec., closely corresponds to the measured time by Salzstein and Pollack (1987). Furthenmore, using this model, the magnitude of the predicted fluid induced shear stresses, 8-30 dynes/cm2, is shown to be similar to the fluid shear stresses measured in osteoblasts and other cells in which an intracellular Ca+ + shear stress response had been observed. This model is also used, in conjunction with anatomical data and the pore fluid pressure relaxation time data, to show that the spacing between the fibers is approximately $7 \mathrm{~nm}$ The result is consistent with the notion that the canalicular pore space is filled with glycosaminoglycans that are ordered by albumin according to the model of Michel (1988). The new hypothesis is also shown to be consistent with the experiments of McLeod et al. (1993) which suggest that high frequency low amplitude postural strains can maintain and even increase bone mass.

355

ACTIVATION OF G-PROTEINS MEDIATES FLOW-INDUCED PROSTAGLANDIN E $E_{2}$ PRODUCTION IN OSTEOBLASTS

J.A. Frangos and K.M. Reich

The Penn State University, University Park, PA 16802

Interstitial fluid flow may play a role in load-induced bone remodelling. Previously we have shown that fluid flow stimulates cyclic adenosine monophosphate (cAMP), inositol trisphosphate $\left(\mathrm{IP}_{3}\right)$ and prostaglandin $\mathrm{E}_{2}\left(\mathrm{PGE}_{2}\right)^{(1,2)}$ production levels in osteoblasts. Flow-induced increases in cAMP and $\mathrm{IP}_{3}$ were demonstrated to be mediated by prostaglandins. $\mathrm{PGE}_{2}$ production may thus be an important component in the signal transduction of fluid flow. In the present study, we will discuss the mechanism of flow-induced $\mathrm{PGE}_{2}$ synthesis.

$\mathrm{PGE}_{2}$ production was mediated by pertussis toxin (PT)-sensitive $\mathrm{G}$ proteins. G-protein activation, in turn, led to the enzymatic release of arachidonic acid leading to $P G E_{2}$ production. Phospholipases $A_{2}$ and $C$ were equally responsible for the total arachidonic acid production. In addition, both extracellular and intracellular calcium were required for full expression of the $\mathrm{PGE}_{2}$ response. These results represent the first clearly defined signal transduction pathways in flow-stimulated osteoblasts. Such pathways may be important in mechanically-induced bone remodelling.
356

OSTEOBLAST-LIKE BONE CELLS RESPOND TO SHEAR STRESS
DUE TO LAMINAR FLOW BY A RISE IN INTRACELLULAR CALCIUM

J.L. Williams, A. Ham, J.P. Iannotti, J. Bleuit, J.H. Chen Department of Orthopaedic Surgery, University of Pennsylvania, Philadelphia, $\mathrm{Pa}$ 19104-6081

Neonatal rat calvarial cells (osteoblast-enriched) were cultured in monolayer on glass slides and subjected to shear stresses of $3,6,31$, and 60 dynes $/ \mathrm{cm}^{2}$ in a laminar flow chamber. The level of intracellular free calcium ion concentration was monitored using Fura-2 fluorescence during the application of stress. Application of a step increase in stress resulted in a transient increase in calcium starting at about 20 seconds, reaching a peak in about 35 seconds and dechining thereafter, despite the continued shear stress, and reaching near baseline values in about 100 seconds. No change in cytoplasmic calcium was observed with a step increase in shear stress of 3 dynes $/ \mathrm{cm}^{2}$, even after a period of ten minutes of stress. Between 6 dynes $/ \mathrm{cm}^{2}$ and 60 dynes $/ \mathrm{cm}^{2}$ the magnitude of the calcium response increased three-fold with the applied shear stress. The average increase in ionized calcium with shear stress for the experiments between 6 and 60 dynes $/ \mathrm{cm}^{2}$ was $2.5 \mathrm{X}$ basal calcium levels.

357

TRANSMEMBRANE POTENTIAL CHANGES IN BONE CELLS DUE TO STRESS-INDUCED EXTRACELLULAR POTENTIALS: A POSSIBLE BONE STRAIN SENSING MECHANISM

Timothy P. Harrigan, James J. Hamilton

Orthopedic Surgery, University of Missouri-Kansas City Medical School

Micro-anatomic studies of calcified bone tissue show gap junctions between osteocytes, and between osteoytes and osteoblasts. These interconnections allow ionic transfer (current exchange) between cells, while collectively insulating them from the extracellular space. The result is a network of cells which function electrically like a much larger cell. At the edge of the network cells can become hyperpolarized or depolarized due to extracellelar electric potential changes. In this study, we assessed this mechanism as a possible stimulus for bone remodeling. We developed a simple model for an array of cells within a poroelastic beam under remote axial and bending loads. Parametric evaluation of the analytical solution for transmembrane potential changes (TPC) showed trends which agree with known data on loading rate sensitivity in bone remodeling. Varying the strength of intercellular coupling due to gap junctions had a complicated effect, however. The surface TPC increased with cellular coupling under axial loading, but not in pure bending. In bending, the surface TPC increases and then decreases to zero as the intercellular coupling is increased. Experimental data from the literature suggests that these cells can be very well connected. This indicates that measurement of the electrical coupling between bone cells can have important implictions for the reaction of bone to mechanical loading. If the cells are well connected, then cancellous bone struts seem to be able to sense axial loads but not bending loads using this mechanism. 
359

CURRENT CONCEPTS IN BIOACTIVE CERAMIC COATINGS

John F. Kay, Ph. D.

Bio-Interfaces, Inc., San Diego, CA

High quality $\mathrm{HA}$-coatings are biostable and withstand functional in-vivo loads, when a proper implant design is used in consideration of properties of the coating metal system. After initial research studies $10+$ years ago, $\mathrm{Ca} / \mathrm{P}$ (HA) coatings are available for orthopaedic joint replacement devices. Performance of some coatings has been shown to be greater than on others, primarily based on differences in coating characterizations and quality controls begun during their application. Data indicate high quality HAcoatings aid in the placement of joint replacement devices and non-precision implants, minimizing lysis around the distal portion of a joint replacement prosthesis, if HA-coatings remain intact and are not dissolved by the body over time. While much is known about fabrication of high quality HA-coatings, there will be future compositional modifications to further control and enhance the biological response so that bone will respond to the placement of joint replacement devices in a more controlled fashion.

360

CHARACTERIZATION OF HYOF COMBUSTION SPRAYED HYOROXYAPATITE COATINGS

J. Haman", L. Lucas*, D. Crawmert

Department of Biomedical Engineering, School of Engineering,

University of Alabama at Birmingham

Miller Thermal, Inc., Appleton, Wiscons in Plasma-arc spraying is the commercial method for producing
hydryoxyapatite (HA) coatings on orthopaedic and dental implants. The purpose of this study was to examine a relatively new coating process, High Velocity Oxy-Fuel (HVOF) combustion spraying, and determine if it could be sucessfully used for the production of bioceramic coatings. Fourier Transform Infrared spectroscopy (FTIR), X-ray Diffraction (XRD), and Scanning Electron Microscopy (SEM) were used to evaluate the chemical, structura 1, microstructura 1, and topographical features of both the HA starting powders and as-sprayed coatings. XRD patterns and lattice parameters revealed some loss in crystallinity of the coating due to the spraying process. FTIR results showed a partial loss of the $\mathrm{OH}^{-}$groups during spraying, however the phosphate groups were still present. SEM analys is showed a lame Tlar structure similar to that caused by plasma spraying but with less cracking and porosity. Although this coating process has not been opt imized, initial results indicate that it may be a viable process for producing bioceramic coatings.

This research is funded in part by the National Science Foundation.

\section{6}

ELECTROCHEMICAL CHARACTERIZATION OF METALLIC IMPLANT MATERIALS

P. Kovac

Orthopaedic Research Department, Smith \& Nephew Richards Inc., Memphis, TN 38116

It has long been recognized that the electrochemical properties of implant metals and alloys have a very significant bearing both on the biocompatibility and the long-term overall performance of metallic biomedical implants. While traditional cortosion testing of various metallic materials clearly identified the most suitable metals and alloys for temporary or permanent implant applications in the harsh biological environment, recent developments in electrochemical experimentation have made it possible to gain a much deeper understanding of relevant electrochemical performance issues. Electrochemical Impedance Spectroscopy (EIS), for instance, bas emerged as a very powerful tool for studying the kinetics of spontaneous passivation, and for distinguishing between the major electrochemical characteristics of highly protective passive films, which is quite difficult to do by other methods. The main objective of this paper is to provide a coherent and logical treatment of the subject of complex electrochemical characterization of metalic implant materials, and to demonstrate that the combination of appropriate theoretical and experimental considerations may result not only in the development of more relevant in vitro experimental considerations may result not only in the development of more relevant in vitro
expents, but may also help substantially with the proper interpretation of clinical findings. Furthermore, a more comprehensive electrochemical approach to the use of metallic materials in the human body is of primary importance because of its essential role in the optinization of the overall eiectrochemical performance of both existing and recenty developed implant alloys. As an example, the compositional and dimensional stability of passive films on currenty used alloys can be significantly improved by a new passivation method. Based on the chemical and electrochemical fundamentals of implant metal biocompatibility, new implant alloys can also be developed, which - with certain surface modifications if desirable - may be expected to perform optimally in various implant applications, including those of orthopaedic, cardiovascular, and dental implant devices. With regard to the long-term performance of an implant device, it is another very important objective of this paper to point out that the electrochemical properties of the materials alone may not be sufficiently predictive without the proper design-, and manufacturing-related considerations.
362

EFFECT OF METAL IONS ON OSTEOGENIC BONE MARROW CULTURES

W.W. Huh and D.A. Puleo

Center for Biomedical Engineering, University of Kentucky, Lexington, KY

Metal ions are released from orthopedic implants into the physiological environment by electrochemical events. The effects of these species on nearby bone and on distant tissues, e.g., liver and spleen, remain largely unknown. The purpose of this study was to ex the effects of metal ions on bone marrow stromal cell cultures that possess the ability to form bone-like tissue in vitro. Bone marrow was harvested from the femurs of adult rats and cultured in $\alpha \mathrm{MEM}$ with $15 \% \mathrm{FBS}, 50 \mu \mathrm{g} / \mathrm{ml}$ ascorbic acid, $10 \mathrm{mM}$ B-glycerophosphate, and $10^{-8} \mathrm{M}$ dexamethasone. The metal ions used were those found in commonly used orthopedic implants, i.e., Ti-6 Al-4V, $\mathrm{Co}-\mathrm{Cr}-\mathrm{Mo}$, and $316 \mathrm{~L}$ stainless steel. Solutions of individual ions and combinations mimicking the alloy composition were prepared from atomic absorption standards and added to the cultures to give concentrations ranging from $50 \mathrm{ppm}$ to $50 \mathrm{ppb}$. After a 48 hour period of exposure to the ions, the bone marrow cultures were examined for cytotoxic responses. Gross toxicity, reflected by cell death, was determined with a methylene blue staining assay, and more subtle toxicity, reflected by decreased activity of mitochondrial dehydrogenases, was ascertained using an MTT assay. Co, Ni, and Mn induced cell death at concentrations down to $5 \mathrm{ppm}$; Co-Cr-Mo and 316L stainless steel elicited cytotoxic responses down to $2.5 \mathrm{ppm}$; and $\mathrm{Cr}_{\mathrm{r}}$ caused cell death down to $500 \mathrm{ppb}$. $\mathrm{Co}-\mathrm{Cr}-\mathrm{Mo}$ inhibited mitochondrial activity at concentrations down to $1 \mathrm{ppm}$; $\mathrm{Cr}$ suppressed mitochondrial activity down to $250 \mathrm{ppb}$. These results show that metal ions associated with $\mathrm{Co}-\mathrm{Cr}-\mathrm{Mo}$ and $316 \mathrm{~L}$ stainless steel are toxic to osteogenic bone marrow cultures at concentrations approximating those measured in the fibrous membrane encapsulating loose implants.

\section{3}

\section{OSTEOBLAST MINERALIZATION ON ORTHOPEDIC BIOMATERLALS} Matthew W. Squire, John L. Ricci*, and Rena Bizios

Dept. of Biomedical Eng., Rensselaer Polytechnic Institute, Troy NY 12180 -Dept. of Bioengineering, Hospital for Joint Disease, New York NY 10003 Formation of new bone by osteoblasts at the tissue-biomaterial interface is considered essential for the success of orthopedic/dental implants. Ti6AI4V, $\mathrm{CoCrMo}$, and 316 stainless steel alloys were polished $(0.3 \mu \mathrm{m}$ finish) and prepared for cell culture according to standard biomaterial protocols. Osteoblasts were then seeded on these metals and cultured in Dulbecco's Modified Eagle Medium supplemented with $10 \%$ fetal bovine serum, $10 \mathrm{mM} \beta$-glycerophosphate, and 50 $\mu \mathrm{g} / \mathrm{ml}$ ascorbate for $18,19,20,21,24$, and 28 days under standard tissue culture conditions. Osteoblasts were fixed according to standard scanning electron microscopy protocols and subsequently analyzed using secondary electron imaging (SEI), back scattered electron imaging (BSEI), and X-ray energy dispersive elemental analysis (EDX). Following 18-28 days of culture, examination of the cellular accumulation on the metals via SEI revealed aggregated spheroidal mineral deposits which appeared electron dense via BSEI. These mineral deposits were located underneath or between osteoblasts and grew progressively larger with time of culture. After $\mathbf{2 4}$ days of culture, the mineral deposits were often associated with collagen fibers. EDX and SEI analyses demonstrated a mineralizing extracellular matrix containing sulphur (S), calcium (Ca), and phosphorous (P) as well as spheroidal mineral deposits containing high amounts of $\mathrm{Ca}$ and $\mathrm{P}$. Following 28 days of culture, spheroidal deposits of $C a$ and $P$ were observed to be situated on and around regularly oriented collagen fibers. This in vitro study demonstrates that osteoblast mineralization on orthopedic/dental biomaterials is a multiphase process involving the deposition of a sulphated extracellular matrix, calcium phosphate, and collagen.

\section{4}

STRESS CORROSION CRACKING RESISTANCE OF Ti-13Nb-13Zr

Ajit K. Mishra and James A. Davidson

Smith and Nephew Richards, Memphis, TN 38116

$\mathrm{Ti}-13 \mathrm{Nb}-13 \mathrm{Zr}$ (Ti-13-13) is a new Titanium alloy developed to meet the requirements for an ideal implant material: low modulus, optimal biocompatibility and superior wear resistance. When Ti-13-13 is diffusion hardened (DH) a wear-resistant ceramic surface is produced. The proprietary, low temperature porous coating developed for this alloy has excellent pull-off strength $(8,780 \pm 620 \mathrm{psi})$ and optimum pore size $(385 \pm 64 \mu \mathrm{m})$ and $\%$ porosity $(48 \pm 1 \%$ ). The present paper describes the results of a slow strain rate test (ASTM STP 665 ) to determine the stress corrosion cracking resistance of Ti-13-13, both with and without diffusion hardening and porous coating, and several other implant alloys. The specimen gage length was 1 inch and the crosshead motion rate was $4 \times 10^{-6} \mathrm{in} / \mathrm{s}$. The time to fracture $\left(t_{f}\right) \%$ elongation $(e)$ and \% reduction in area $(R)$ were measured for each specimen in Ringer's solution at $100^{\circ} \mathrm{F}$, and compared with the corresponding values in tests at ambient temp. in air. Visual examination was made at $20 x$ for evidence of environmental or secondary cracking. The results are given below:

\begin{tabular}{|c|c|c|c|c|c|c|}
\hline & $\begin{array}{l}\text { \# of } \\
\text { tests }\end{array}$ & $\left(t_{f}\right) /\left(t_{f}\right)_{\text {air }}$ & (e)/(e) air & $(\mathrm{R}) /(\mathrm{R})_{\text {air }}$ & $\begin{array}{l}\text { Crack } \\
\text { Environ. }\end{array}$ & $\begin{array}{l}\text { ing? } \\
\text { Secondary }\end{array}$ \\
\hline Ti-6Al-4V & 5 & 1.04 & 1.04 & 0.99 & No & No \\
\hline 316L S.S. & 5 & 0.99 & 0.98 & 1.00 & No & No \\
\hline $\mathrm{F}-799 \mathrm{Co}-\mathrm{Cr}$ & 5 & 1.05 & 1.06 & 1.13 & No & No \\
\hline Ti-13-13 & 5 & 1.01 & 0.99 & 1.26 & No & No \\
\hline DH Ti-13-13 & 5 & 0.95 & 0.90 & 1.00 & No & No \\
\hline Porous Ti-13-13 & 5 & 1.02 & 0.98 & 1.03 & No & No \\
\hline
\end{tabular}
and in environment, and no evidence of stress corrosion cracking, in any of the materials even in this severe test. 
365

PREDICTION OF LONG-TERM STAESS RELAXATION BEHAVIOR OF ACRYLIC BONE CEMENTS USING THE TIME TEMPERATURE SUPERPOSITION PRINCIPLE. D. N. Yetkinler and A. S. Litsky

Orthopaedic BioMaterials Laboratory, The Ohio State University

A reduced-modulus bone cement was developed to lower the contact stresses at the bone-cement interface and thereby reduce the resorptive modeling which initiates the losening of the cemented total joint components. The reduced-modulus formulation. PBMMA, consisting of polybutylmethacrylale beads in a methylmethacrylate matrix, has an elastic modulus of $0.27 \mathrm{GPa}$ at body temperature vs $2.1 \mathrm{GPa}$ for standard polymethylmethacrylate, PMMA, bone cement. Pilot in vivo studies have verified the effectiveness of the PBMMA in decreasing femoral stem loosening, as measured radiographically and mechanically. The use of viscoelastic materials in weight-bearing applications raises the concern of prosthesis subsidence due to creep. Since the expected life span of bone cements are more than 10 years, it is important to determine both long-term and short-term viscoelastic propertie

Short-term, 100 hours, unconstrained stress relaxation tests have been performed at $1 \%$, $2.5 \%$, and $5 \%$ strains. Results indicate a rapid initial stress redistribution followed by slow steady-state decrease in stress at a rate equivalent to that found in PMMA. To investigate long-term viscoelastic behavior of both ant levigat eformed amorpos. chape inding that time and shape, indicaling that time and temperalure have an equivalent effect on the elastic modulus. The data indicates a slow steady-state stress relaxation in PBMMA up to 10 years.

366

ACETABULAR DESIGN CONSIDERATIONS FOR THE MINIMIZATTON OF POLYETHYLENE WEAR DEBRIS

D. M. Ryan, J. J. Shea

Snith \& Nephew Richards Inc.

Progressive osteolysis, a probable result of small particle polyethylene debris, is a major concern in total hip arthroplasty today. Large quantities of polyethylene debris from modular acetabular components are reported to create adverse biological responses that result in implant loosening and ultimately implant failure. Research bas proven that one area where this debris is being generated is at the femoral head/liner interface. Much progress has been made in this area with the introduction and evolution of ceramic femoral heads. More recently, it has been noted that polyethylene debris can also be generated at the liner/shell interface. Two factors contributing to the amount of debris generated at this liner/sbell interface. Two factors contributing to the amount of debris generated at this interface are the surface characteristics of the inside surface of the acetabular shell and the addressed through design. This investigation quantifies these surface characteristics affecting debris formation.

In this investigation, eight (8) acetabular component designs were evaluated. Average surface roughness $(\mathrm{Ra})$ measurements were taken on each shell using a Tokyo Seimitsu Surfcom 570 Profilometer. Surface characteristics were evaluated with the use of LeicaCambridge Stereoscan 360 Scanning Electron Microscope. The results showed that, between manufacturers, there were average surface roughness (Ra) differences up to $80 \mathrm{X}$ found
367

ACETABULAR LINER MICROMOTION AS A RESULT OF APPLIED TOROUE R.D. Lambert, T.W. McLean Smith \& Nephew Richards Inc.

Tests developed to measure torque load to failure of acetabular liners have been historically measured in a static mode. Clinically, this kind of failure is typically described as disassociation or mechanical failure of the device. Failure of the device may also be attributed to debris induced osteolysis which can occur as a result of particulate generation at the articulating surface of the femoral head and liner and at the nonarticulating interface of the liner and shell. Particulate generation between the nonarticualting surfaces can be minunized for modular acetabuiar components through design. This investigation attempts to quantify the rotational displacement between the liner and shell under phsyiological cyclic torque loads as well as identify cumulative damage occurring at the liner/shell interface.

In this investigation two acetabular component designs were evaluated. Cyclic torque loads were applied directly to the liner of assembled acetabular shells for 3.5 million cycles. An LVDT measured the rotational displacement of the liner with respect to the shell. The results showed that design A rotated a maximum of $0.025^{\circ}$ in cyclic micromotion. Design B resulted in a maximum of $0.7^{\circ}$ of cyclic micromotion.

368

THE ROLE OF GROWTH FACTORS IN OSTEOBLAST INTERACTIONS WTH PEPTIDE-MODIFIED SURFACES

Kay C Dee, Thomas T. Andersen*, Frank A. Blumenstockt, Rena Bizios

Dept. of Biomedical Engineering, Rensselaer Polytechnic Institute,

Troy, NY, 12180-3590; "Dept. of Biochemistry, Albany Medical College;

Albany, NY, 12208, †Dept. of Physiology, Albany Medical College, Albany, NY, 12208

While adhesive and migratory behavior of anchorage-dependent cells is regulated by the interaction of integrin receptors with speciffc adhesive peptide sequences, the presence of external chemical stimuli such as growth factors may alter expected cell/substrate interaction patterns. We have investigated the effects of growth factors such as fibroblast growth factor, transforming growth factor-beta, and insulin-like growth factor on the adhesion. morphology, proliferation, and motility of well-characterized neonatal rat calvarial osteoblast behavior on surfaces modified with chemically immobilized peptide sequences. The material surface was prepared by covalently bonding the adhesive peptides RGDS (Arg-Gly-Asp-Ser), YIGSRG (Tyr-Ile-Gly-Ser-Arg-Gly), and the inactive control RGDS (Arg-Asp-Gly-Ser) to aminophase glass coverslips. The results promote understanding of the complex cellular/molecular interactions at the bone/implant interface, and may be utilized in the design of new biomaterials which elicit desirable biological responses from surrounding tissues. 
369

FUZZY CONTROL OF AN ELECTRICAL MUSCLE STIMULATIONRECIPROCATING GAIT ORTHOSIS SYSTEM FOR SPINAL CORD INJURED

David B. Reynolds, Kuldip S. Rattan and Chandler A. Phillips

College of Engineering and Computer Science, Wright State University, Dayton, $\mathrm{OH}$

To ald the spinal cord injured (SCI) back into the activities of daily fiving, including the work environment, invesligators at our institution have developed a minimally invasive system to restore basic motor function to the lower limbs. The system is a hybrid electromechanical one, using electronic muscle stimulators (EMS) for functional electrical stimulation (FES), an electrode delivery system using a transcutaneous transducer gament (TTG) and a reciprocating gait orthosis (RGO). After physical conditioning of the subject, this system has allowed both quadriplegic and paraplegic subjects to stand, sit and walk forward and backward. In most of the studies, FES parameters were controlled by the subject using only visual information as feedback. The design of a closectloop controller for this complex application can not be accomplished using standard proportional, integral and derivative control. Not only does the stimulating electrode interface differ from subject to subject, but it differs for the same subject over time. This problem is fertlle ground for fuzzy control, which is a rule-based control. The range of the error signal (desired jolnt angle/velocity actual joint angle/velocity) is divided up into a number of intervals which are assigned gains based upon rules delermined experimentally in this case. Thus gain may depend upon both magnitude and sign of the error. Fuzzy control has been shown to provide fast rise time with little or no overshoot in complex systems. We intend to modify the RGO to provide feedback of RGO joint angle/velocity at ths hip and kree for use in a fuzzy logic controller. We then plan to study fuzzy-controlled stand-up and sit-down with this system in SCI subjects. Current EMS- or FES-RGO systems are used primarily in outpatient physical therapy in rehabilitation clinics. The concept of fuzzy control in a closed-toop, sublect initiated system offers the possibility of moving this lechnology to the workplace, where the person may gain both health and economic benefits from its daily use.

370

THE APPLICATION OF SOLID MODELLING AND COMPUTER ANALYSIS IN THE DESIGN OF PRODUCTS FOR REHABILITATION ENGINEERING

*ROBERT BROWN, **DAVID K WRIGHT

*FACULTY OF ART, DESIGN AND TECHNOLOGY, CIHE, WALES, UK. **DEPARTMENT OF DESIGN, BRUNEL UNIVERSITY, UK.

The design and manufacturing process when developing products for the elderly and disabled is complex and expensive because the interface between user and device is of ten not well defined and subject to considerable variation. The main reasons for this being the range and variability which exists within a particular disability or medical condition.

Traditional product development and manufacturing processes in many cases prove inappropriate and prohibitively expensive as the designer/product engineer might need to produce several variations of a prototype before a satisfactory user 'fit' is achieved.

We have developed a systems based approach to rehabilitation engineering design in which the user product interaction is emphasised. This computer based approach to problems relating to special human need makes use of analysis software for mechanical interaction. Designing within a solid modelling system enables product interaction with an andriod to take place. This enables the design engineer to analyse and evaluate products within a computer system before producing physical prototypes.
371

COMPUTER ACCESS VIA SWITCH BASED MOUSE O'Connell M.J.

Cardiff Institute of Higher Education, Wales, UK.

Many physically disabled individuals are denied access to computer software because of their inability to use a keyboard or mouse in the normal way. Adequate access to standard commercial software would radically improve opportunity for employment.

A 'Switchmouse' has been developed which emulates all the functions of conventional mouse but without the need for precision hand movement. It gives the majority of disabled users, including those with only the barest pointing ability, access to a wealth of commercially available software. it is particularly effective with mouse controlled graphics packages and Graphical User Interfaces (GUI). The Z8 microcontroller based device provides both parallel and serial mouse facilities making it compatible with most IBM PC's and clones. All mouse functions are available. A simple display allows the user to monitor the status of the 'mouse'. No modification to the original software or mouse driver is required.

The Project is part of a collaborative research programme linking the Electronic Engineering and Speech and Language Therapy sections of Cardiff Institute of Higher Education, and Craig-y-Parc Spastic Society School for cerebral palsied children. 